$\mathrm{SLAC}-342$

$\mathrm{UC}-34 \mathrm{D}$

(E)

\title{
A SEARCH FOR A CLOSE-MASS LEPTON DOUBLET*
}

\author{
John Keith Riles \\ Stanford Linear. Accelerator Center \\ Stanford University \\ Stanford, California 94309 \\ April 1989 \\ Prepared for the Department of Energy \\ under contract number DE-AC03-76SF00515
}

Printed in the United States of America. Available from the National Technical Information Service, U.S. Department of Commerce, 5285 Port Royal Road, Springfield, Virginia 22161. Price: Printed Copy A08, Microfiche A01.

* Ph. D. thesis 


\begin{abstract}
Described is a search for a heavy charged lepton with an associated neutrino of nearly the same mass, together known as a close-mass lepton doublet. The search is conducted in $\mathrm{e}^{+} \mathrm{e}^{-}$annihilation data taken with the Mark II detector at a center-ofmass energy of $29 \mathrm{GeV}$. In order to suppress contamination from conventional twophoton reactions, the search applies a novel, radiative-tagging technique. Requiring the presence of an isolated, energetic photon allows exploration for lepton doublets with a mass splitting smraller than that previously accessible to experiment. No evidence for such a new lepton has been found, enabling limits to be placed on allowed mass combinations. Mass differences as low as $250-300 \mathrm{MeV}$ are excluded for charged lepton masses up to $10 \mathrm{GeV}$.
\end{abstract}




\section{Acknowledgments}

I owe much to my fellow Mark II/PEP5 collaborators. Withont their dedication and care in building and operating the detector, my own work would have been impossible. In addition, the analysis described here benefited from the superb work of those who preceded me. I am grateful to all who contributed to the Mark II effort.

I would especially like to thank my three advisors: Bob Hollebeek gave me wise advice during my first years at SLAC; Jonathan Dorfan helped guide me toward my first publication and has provided unwavering encouragement and support; Martin Perl conceived the original idea for this thesis, and his enthusiasm and advice throughout the work described here have been greatly appreciated. I also thank Fred Gilman and Bennie Ward for helpful conversations and for their patience with my ignorance.

Much that I have learned of high energy physics, I learned from fellow graduate students. I thank Spencer Klein, Rene Ong, and Tom Steele for many useful discussions. Even more, I would like to thank Dean Karlen not only for sharing lodgings for many years, but also for his acute insight, from which my own work often profited. Bruce LeClaire deserves special mention for his readiness to argue me into the ground until either he convinced me he was right or until I convinced him I was right, at which point I knew I must be right.

I have enjoyed working with the new Mark II collaborators in the SLC effort. In particular, I have gained much from close work with Tim Barklow, whose dedication has been inspiring. Thanks go to all my co-workers in SLAC Group C for their support and camaraderie, cspecially to my office-mate, Charlotte Hee. Playing weekly with the volleyball gang has been a welcome diversion from physics.

These last few years of graduate school have been happy ones, thanks to Dolly Wu's humor and affection. Finally, most of all, I want to thank my parents for their patience and their love, without which I would never have made it this far. 


\section{Table of Contents}

Abstract $\quad$ iii

Acknowledgements $\quad$." iv

Table of contents $\quad \mathrm{V}$

List of tables viii

List of figures _ - . _ ix

CHAPTER 1 Introduction 1

...1.1 The Standard Model 3

1.2 Extending the Standard Model 5

1.3 Searching for the Fourth Generation 6

1.3.1 Traditional approaches . . 6

1.3.2 Results of previous searches 11

1.3.3 Radiative tagging 12

1.4 Outline of Thesis 16

CHAPTER 2 Close-Mass Lepton Properties 17

$\begin{array}{lll}2.1 & \text { Weak Interactions } & 17\end{array}$

2.2 Calculation of Heavy Charged Lepton Decay Widths 21

2.3 Lifetime Considerations 25

CHAPTER 3 Radiative Production of Heavy Leptons 30

3.1 Non-radiative Lepton Production 30

3.2 Single Photon Lepton Production 31

$\begin{array}{lll}3.2 .1 & \text { Bremsstrahlung } \quad \text { - } & 31\end{array}$

3.2.2 Radiative corrections $\quad 37$

3.3 Double Photon Lepton Production 43

3.4 Weak Effects $\quad 45$

3.5 Monte Carlo Corrections 47 
CHAPTER 4 PEP and the Mark II Detector 53

4.1 The PEP Storage Ring 53

4.2 Overview of Mark II Detector 54

4.3 Vertex Chamber 56

4.4 Outer Drift Chamber $\quad 57$

$\begin{array}{lll}4.5 & \text { Time-of-Flight Counters } & 59\end{array}$

4.6 Magnetic Coil 60

4.7 Electromagnetic Calorimeter 61

4.8 Outer Steel and Proportional Tubes 66

4.9 Small Angle Tagger 66

4.10 Trigger Considerations $\quad 67$

CHAPTER 5 Analysis and Results 73

5.1 Event Selection 73

$\begin{array}{ll}\text { 5.1.1 Charged track requirements } & 73\end{array}$

$\begin{array}{lll}5.1 .2 & \text { Tagging photon requirements } & 75\end{array}$

5.1.3 Event topology requirements - 76

$\begin{array}{lll}5.1 .4 & \text { Fvent veto conditions } & 80\end{array}$

5.2 Background Estimation $\quad 90$

$\begin{array}{ll}\text { 5.2.1 Radiative } \tau \text { background } & 91\end{array}$

$\begin{array}{lll}\text { 5.2.2 Two-photon } \tau \text { pair production } & 91\end{array}$

$\begin{array}{lll}\text { 5.2.3 Two-photon production of four pions } & 92\end{array}$

5.2.4 Hadronic event production $\quad 93$

5.3 Efficiency Corrections for Detector Simulation 94

5.4 Comparison of Data to Background Estimate 96

5.5 Limits on a New Heavy Lepton Doublet 99

$\begin{array}{ll}5.6 \text { Conclusions and Outlook } & 111\end{array}$

APPENDIX A Lepton Production and Decay Simulation 117

$\begin{array}{lll}\text { A.1 Production Simulation } & 118\end{array}$ 
$\begin{array}{ll}\text { A.2 Event Generation Algorithm } & 125\end{array}$

A.3 Decay Simulation $\quad 126$

A.3.1 Fermionic decay (3-Body) 126

A.3.2 Scalar decay (2-Body) 128

A.3.3 Vector decay (2-Body) 129

A.3.4 Vector decay (3-Body) - 132

A.3.5 Quark decay (total partial width) 134

$\begin{array}{lll}\text { A.3.6 Branching ratios } & 137\end{array}$

REFERENCES 143 


\section{List of Tables}

3.1 Estimated radiative corrections to bremsstrahlung production 49

$\begin{array}{lll}5.1 & \text { Efficiency corrections for inaccurate detector simulation } & 97\end{array}$

5.2 Final background estimates 97

5.3 Electron/pion distributions for surviving events 101

5.4 Confidence levels for exclusion of Monte Carlo samples $(\delta \mathrm{M}=0.2-0.4) \quad 108$

5.5 Confidence levels for exclusion of Monte Carlo samples ( $\delta \mathrm{M}=0.4-0.6) \quad 110$

5.6 Confidence levels for exclusion of Monte Carlo samples $(\delta \mathrm{M}=0.8-1.2) \quad 112$

5.7 Confidence levels for exclusion of Monte Carlo samples $(\delta \mathrm{M}=1.4-2.0) \quad 113$

A.1 Tau branching ratios used by LULEPT 139

A.2 Tau efficiency corrections for LULEPT decay modes 140 


\section{List of Figures}

1.1 Feynman diagram for $\mu^{-} \rightarrow \nu_{\mu} \mathrm{W}^{-}$. 4

1.2 Feynman diagram for heavy neutrino magnetic moment 7

1.3 Feynman diagram for electron-muon production through heavy leptons 7

1.4 Distribution in total energy of events with two charged particles 9

1.5 Feynman diagrar for general two-photon process 9

1.6 Current limits on existence of heavy leptons 12

1.7 Feynman diagram for radiative heavy lepton production 13

$\begin{array}{lll}1.8 & \text { Feynman diagram for radiative two-photon process } & 14\end{array}$

1.9 Feynman diagrams for $\mathrm{e}^{+} \mathrm{e}^{-} \rightarrow \mathrm{e}^{+} \mathrm{e}^{-} \mu^{+} \mu^{-}$production $\quad 15$

$\begin{array}{ll}2.1 \text { Feynman diagram for four-fermion weak interaction } & 19\end{array}$

$\begin{array}{ll}2.2 \text { Modern Feynman diagram for a weak interactions } & 19\end{array}$

2.3 Feynman diagram for general heavy lepton decay 21

2.4 Heavy lepton branching ratios vs mass difference - 24

2.5 Average decay length of heavy charged lepton vs mass difference 27

2.6 Definition of decay product impact parameter 28

2.7 Root-mean-square of $\sin \theta$ of decay product pion direction 28

2.8 Average impact parameter of decay pion vs mass difference 29

3.1 Lowest order process for charged lepton production 32

3.2 Lowest order bremsstrahlung production of lepton pair : 34

3.3 Photon energy spectrum for initial and final state radiation 37

3.4 Feynman diagrams for general initial state bremsstrahlung 38

3.5 Feynman diagrams for initiäl state virtual corrections 39

3.6 Additional processes included in exact radiative correction 42

3.7 Total radiative corrections (initial and final) to $\mathrm{e}^{+} \mathrm{e}^{-} \rightarrow \mathrm{L}^{+} \mathrm{L}^{-}$

3.8 Weak contribution to lowest order production of charged lepton pair 47 
3.9 Distributions in final state radiation event weight correction 48

3.10 Estimated radiative correction to bremsstrahlung production 50

3.11 Final normalizing cross section for radiative lepton production $\quad 52$

4.1 The Mark II detector $\quad 55$

4.2 Wire pattern of vertex chamber inner layers $\quad 57$

4.3 Time-of-flight acceptance. $\quad \cdots$

4.4 Ganging scheme for liquid argon calorimeter channcls $\quad 62$

4.5 Exploded view of liquid argon calorimeter module 63

4.6 Detection efficiency for electromagnetic showers vs $\phi$

4.7 Detection efficiency for electromagnetic showers vs $\theta$

4.8 Detection efficiency for low energy- electron showers $\quad: \quad 66$

4.9 Side view of small angle tagger(SAT) $\quad 67$

4.10 Definition of hardware trigger track 69

4.11 Example of trigger asymmetry for high-impact-parameter tracks. 71

4.12 Trigger track efficiency for $K_{\mathrm{s}}^{0}$ tracks 72

5.1 Distribution in charged track momenta. $\quad 75$

5.2 Distributions in total neutral energy near to the tagging photon $\quad 77$

5.3 Distribution in opening angle between charged tracks 78

5.4 Distribution in non-tagging neutral energy $\quad 79$

5.5 Distribution in total SAT energy background 81

5.6 Distribution in SAT energy background (coincidence-latched) 82

5.7 Sample cosmic ray coincidence event (1) 83

5.8 Distribution in excess unassociated LA energy 86

5.9 Sample cosmic ray coincidence event (2a) 87

5.10 Sample cosmic ray coincidence event $(2 \mathrm{~b}) \quad 89$

5.11 Distribution in tagging photon energy 98

5.12 Distribution in invariant mass of charged pair 99

5.13 Distribution in invariant mass of charged-neutral pairs 100 
5.14 Distribution in total energy

5.15 Distribution in single charged track momentum

5.16 Distribution in tagging photon energy(with signal)

5.17 Distribution in invariant mass of charged pair(with signal)

5.18 Distribution in invariant mass of charged-neutral pairs(with signal) 103

5.19 Distribution in total energy(with signal)

5.20 Distribution in single charged track momentum(with signal)

5.21 Generated Monte Carlo event samples for heavy lepton production

5.22 Exclusion contour at $95 \%$ confidence level

5.23 Exclusion contours at $95 \%$ and $99 \%$ confidence levels

-. 5.24 Photon energy spectrum for c.m. energy $4 \mathrm{GeV}$ above $\mathrm{Z}$ mass

A.1 Coordinate system for evaluation of spin coefficients

A.2 Feynman diagrams for $\mathrm{L}^{-} \rightarrow \nu_{\mathrm{L}} \mathrm{V}^{-}$and $\mathrm{e}^{+} \mathrm{e}^{-} \rightarrow \mathrm{V}^{0}$

A.3 Illustration of spin suppression in $\tau^{-} \rightarrow \nu_{\tau} \rho^{-}$

A.4 Feynman diagrams for leptonic and hadronic decays

A.5 Branching ratios vs mass difference $\left(\mathrm{M}_{\mathrm{L}}=5,10,14.5 \mathrm{GeV}\right.$

A.6 B.R. vs mass difference $\left(\mathrm{M}_{\mathrm{L}}=10 \mathrm{GeV}\right.$, magnified)

A.7 B.R. vs mass difference $\left(\mathrm{M}_{\mathrm{L}}=10 \mathrm{GeV}\right.$, further magnified) 


\section{Chapter 1}

\section{Introduction}

The "Standard Model" of elementary particles has been remarkably successful to date in describing nature as we have observed it. No known measurement can be said to disagree significantly and conclusively with its predictions. The shortcomings of the Standard Model lie not in its inaccuracy but in its phenomenological nature. The Model describes and to some extent explains nature, but the explanation is incomplete. Why are there quark and lepton generations? Why are neutrinos apparently massless? These are just two of the many questions the Standard Model leaves unanswered.

There have been various attempts to answer the generation question in the context of Grand Unified Theories but none has been satisfactory. ${ }^{1}$ The very small mass of neutrinos has also been addressed, ${ }^{2}$ but again with no convincing success. This gives the experimentalist hope that the unexpected is not necessarily the impossible.

This paper describes a search in electron-positron annihilation data for the decay products of something unexpected, a heavy charged lepton with an associated stable neutrino of nearly the same mass. . Such leptons, where the difference between the charged and neutral lepton masses is relatively small, have been dubbed "close-mass" by Martin Perl, who pointed out a few years ago that their existence had not yet been excluded by measurement. ${ }^{3}$ Previous searches in proton-antiproton collisions have excluded charged leptons with assumed massless neutrino partners for masses 
up to $\approx 41 \mathrm{GeV},{ }^{* 4,5}$ although such results must be regarded with some reservation because of uncertainties in extracting a heavy lepton from large, poorly understood backgrounds in $p \bar{p}$ data. Searches conducted in $\mathrm{e}^{+} \mathrm{e}^{-}$data have more conclusively excluded charged leptons with masses up to $28 \mathrm{Gev},{ }^{6-8}$ with charged-neutral mass differences as low as $6 \mathrm{GeV}$. In addition, other recent searches in electron-positron data have excluded charged lepton masses up to $\approx 14 \mathrm{GeV}$ for mass differences down to $\approx$ 0.3-1.0 Gev. ${ }^{9,10}$ In probing very small mass differences, however, these searches have been hampered by the low energies of the detectable lepton decay products $(e, \pi$, etc.). These low energies hurt in two ways. The first is that backgrounds from two-photon processes become enormous at lower energies, and at the same time, identification of electrons from calorimetry information and identification of muons from their range in dense matter become unreliable. Poor electron and muon identification prevent applying the most powerful heavy lepton discriminant just when it becomes most necessary.

This search uses a new discriminant to extract the signal for heavy lepton production from the overwhelming two-photon background. The novel requirement is the presence of an isolated, energetic photon as an indication of electromagnetic radiation from the initial state electron or positron(for heavy leptons, final state radiation is less important). Demanding an isolated photon be produced at large angles with respect to the $\mathrm{e}^{+} \mathrm{e}^{-}$beams suppresses the two-photon background much more than it does the heavy lepton signal. The aim of this thesis is to determine whether that suppression of conventional backgrounds is sufficient to allow detection of a new close-mass lepton generation.

* In this work, a system of units is used in which the speed of light c and Planck's constant $\hbar$ are defined to be unity. All masses, momenta, and energies are expressed in the same energy units of electron-volts $(\mathrm{eV}, \mathrm{MeV}, \mathrm{GeV}$, etc.) 


\subsection{The Standard Model}

The Standard Model is a phenomenological description of elementary particles observed in nature and of the forces that govern their interactions. There are three distinct kinds of particles that are currently considered "elementary": quarks, which make up hadrons, including both baryons such as the proton and neutron, and nesons such as the pion and kaon; leptons, of which the electron is the most familiar member; and gauge bosons, which mediate the various interactions.

There are generally believed to be six kinds of quarks: up, down, strange, charge, bottom, and top. None of these particles has been detected directly, and no particle yet discovered is believed to contain the top quark, but the indirect evidence, such as deep inelastic electron and neutrino scattering, the energy dependence of the $\mathrm{e}^{+} \mathrm{e}^{-}$ annihilation cross section, the dramatic resonant particles such as the $\phi$, the $\psi$ and the $\Upsilon$, including their excited states all support our confidence in the validity of the quark model. The fact that quarks have not been directly seen is attributed to the overwhelming strong force acting between them. If we try to tear a quark from its containing hadron in an accelerator experiment, this force "neutralizes" the quark by the creation of a new quark-antiquark pair, one of which binds to the exiting quark. Thus the strong force frustrates our efforts to isolate solitary quarks. The details of this process are not well understand theoretically, but can be explained qualitatively in phenomenological models. ${ }^{11}$

Quarks are normally grouped in pairs, called weak doublets:

$$
\left(\begin{array}{l}
u \\
d
\end{array}\right) \quad\left(\begin{array}{l}
c \\
s
\end{array}\right) \quad\left(\begin{array}{l}
t \\
b
\end{array}\right)
$$

The strong force acts between pairs of quarks through the exchange of gluons. Like quarks, gluons have not been detected directly, but the success of perturbative models of quantum chromodynamics in describing the observed energy dependence of hadron structure functions and, more dramatically, the prediction and subsequent 
discovery of three-jet events in $\mathrm{e}^{+} \mathrm{e}^{-}$annihilation, indicate that gluons do exist and play a critical role in binding quarks.

Leptons do not feel a strong force, but do respond to electromagnetic and weak forces, as the quarks do. Leptons also come in weak doublets:

$$
\left(\begin{array}{c}
e \\
\nu_{e}
\end{array}\right) \quad\left(\begin{array}{c}
\mu \\
\nu_{\mu}
\end{array}\right) \quad \cdots \quad\left(\begin{array}{c}
\tau \\
\nu_{\tau}
\end{array}\right)
$$

As with quarks, the characteristic that determines the pairing is association with the weak-force-mediating $\mathrm{W}^{ \pm}$boson. In the case of leptons, the grouping seems absolute, that is, a muon can turn into a muon neutrino with the emission of a virtual W particle (see fig. 1.1), but it will not turn into an electron neutrino the same way. This property is called lepton flavor conservation. In this search, flavor conservation is assumed to hold for the sought heavy lepton to the extent that possible mixing between the new neutrino and known neutrinos is neglected in calculating decay rates and to the extent that the heavy neutrino does not itself decay within the $\operatorname{detector}\left(\tau_{\nu_{\mathrm{L}}} \geq 100 \mathrm{~ns}\right)$.

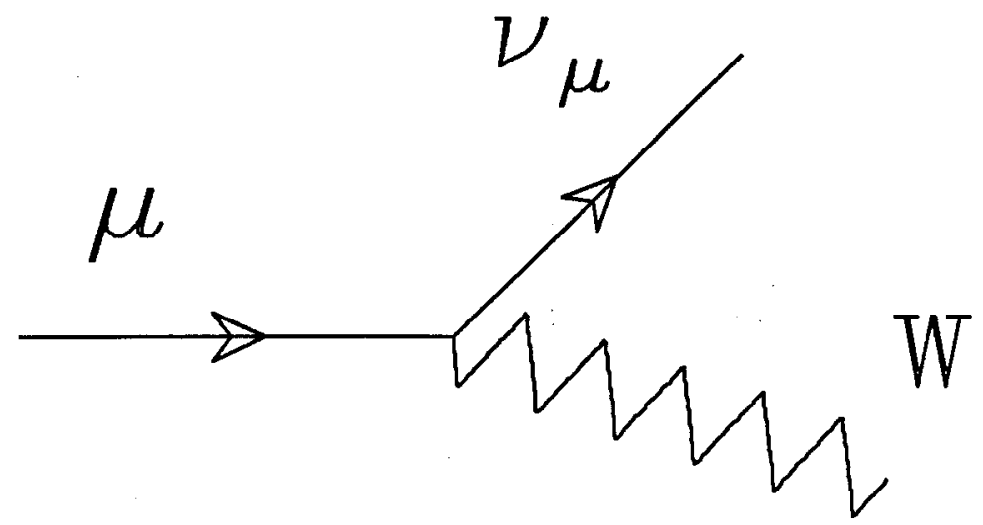

Figure 1.1. Feynman diagram describing the lepton flavor conserving process $\mu^{-} \rightarrow \nu_{\mu} \mathrm{W}^{-}$. The muon decays into a muon neutrino and a virtual $\mathrm{W}$ boson(which itself will further decay). As far as is known today, the muon couples in this way to only its own distinct neutrino, not, for example, to an electron neutrino.

Quark flavor is nearly but not quite conserved. Mixing between quark generations 
occurs, allowing for example $\mathrm{c} \rightarrow \mathrm{uW}^{+}$, along with the expected dominant mode, $\mathrm{c} \rightarrow \mathrm{sW}^{+}$. Such mixing is suppressed, and this suppression is described by the mixing angles defined in the Kobayashi-Maskawa matrix, ${ }^{12}$ angles which are all quite small.

\subsection{Extending the Standard Model}

It is not known whether further generations of quarks or leptons exist. Despite widespread confidence in its existence, even the top quark remains to be discovered. Searches for the lighter member of a fourth generation quark doublet, called $b^{\prime}$, have àlso met with no success.

No direct evidence exists yet for a fourth generation charged lepton. In addition to the conventional approaches using electron and muon identification to search for heavy-lepton decay products, there have been searches made for stable charged leptons, ${ }^{6-8}$ which have excluded masses up to $28 \mathrm{GeV}$. In addition, there have been searches, analogous to this one, that have looked for a single detected photon in $\mathrm{e}^{+} \mathrm{e}^{-}$ collisions as a signal for radiative production of neutrino-antineutrino pairs through $\mathrm{e}^{+} \mathrm{e}^{-}$annihilation into a virtual $\mathrm{Z}$ particle. These searches ${ }^{13-16}$ place an upper limit in the range of 5-7 on the number of conventional massless neutrinos which couple to the weak $\mathrm{Z}^{0}$ boson, and therefore on the number of conventional standard model lepton generations. Cosmology arguments based on the measured current abundance of helium in the universe place an upper limit on the number of neutrinos in the range of $4-6$, depending on various assumptions. ${ }^{17,18}$ Similar arguments, based on the measured total energy density of the universe exclude stable neutrino masses in the approximate range $65 \mathrm{eV}$ to $4 \mathrm{GeV} \cdot{ }^{19-22}$

As mentioned before, the standard model provides no explanation for the apparent masslessness of neutrinos or for the number of observed generations. A priori, one cannot rule out the possibility of a fourth lepton doublet or the possibility that the neutral member of that doublet is heavy. 
Modest theoretical encouragement comes from a model proposed by Raby and West ${ }^{23}$ which postulates a heavy stable neutrino of mass in the approximate range 4-10 Gev with a charged lepton partner that is slightly heavier (by $\leq 1-2 \mathrm{Gev}$ ) than the neutrino. The model attempts to explain the solar neutrino puzzle, i.e., the surprisingly low flux of neutrinos reaching the earth from nuclear reactions occurring in the core of the sun. ${ }^{24,25}$ Stable heavy neutrinos, in this scheme, would lower the temperature of the sun's core by carrying heat energy to larger radii, thus reducing the temperature-sensitive rate of neutrino-producing reactions.

This model could also explain the dark matter problem, the apparent invisibility of roughly $90 \%$ of the universe's mass, ${ }^{26}$ since a relatively low abundance of heavy neutrinos would provide a substantial mass density. Essential to the Raby and West argument is the requirement that the heavy neutrino possess an anomalous magnetic moment, arising from higher order loop processes (see fig. 1.2), involving both the slightly heavier charged lepton and a postulated charged Higgs boson of comparable mass. The magnetic moment is necessary to ensure that the stable neutrino interact significantly with the plasma at the sun's core. The necessary additional assumption of a heavy charged Higgs particle weakens considerably the appeal of this model, despite its claimed resolution of two major outstanding puzzles in astrophysics.

Given our ignorance, however, experimentalists cannot afford complacency as to what is or is not reasonable to expect. With these motivations, let us now consider various approaches to detecting the existence of a fourth generation lepton doublet.

\subsection{Searching for the Fourth Generation}

\subsubsection{Traditional approaches}

Traditionally, the most powerful approach in searching for a new heavy lepton doublet in $\mathrm{e}^{+} \mathrm{e}^{-}$annihilation has been the requirement of one electron and one muon (see fig. 1.3), with no other detected particles in a single $\mathrm{e}^{+} \mathrm{e}^{-}$annihilation "event." 


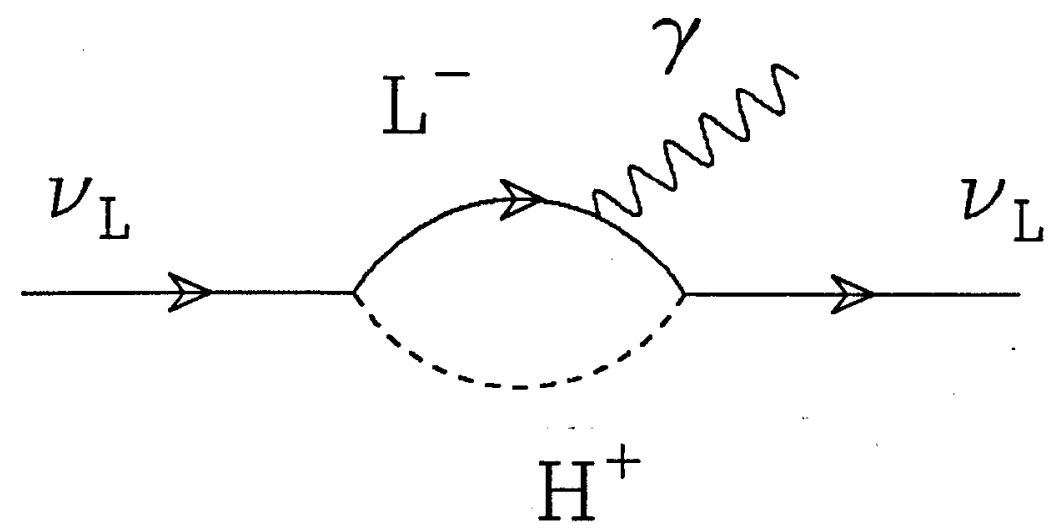

Figure 1.2. Feynman diagram describing a process that imparts an anomalous magnetic moment to the heavy neutrino proposed by Raby and West. If the charged lepton and charged Higgs particle ... are both slightly heavier than the neutrino, this diagram leads to a large magnetic moment.

Since two neutrinos and two antineutrinos leave undetected, there is usually substantial missing energy in these events. This electron-muon tag led to the original discovery of the tau lepton, ${ }^{27}$ at $\sqrt{\mathrm{s}}$ in the range $4-7 \mathrm{Gev}$ and remains a powerful discriminant in many higher energy searches for heavy leptons.

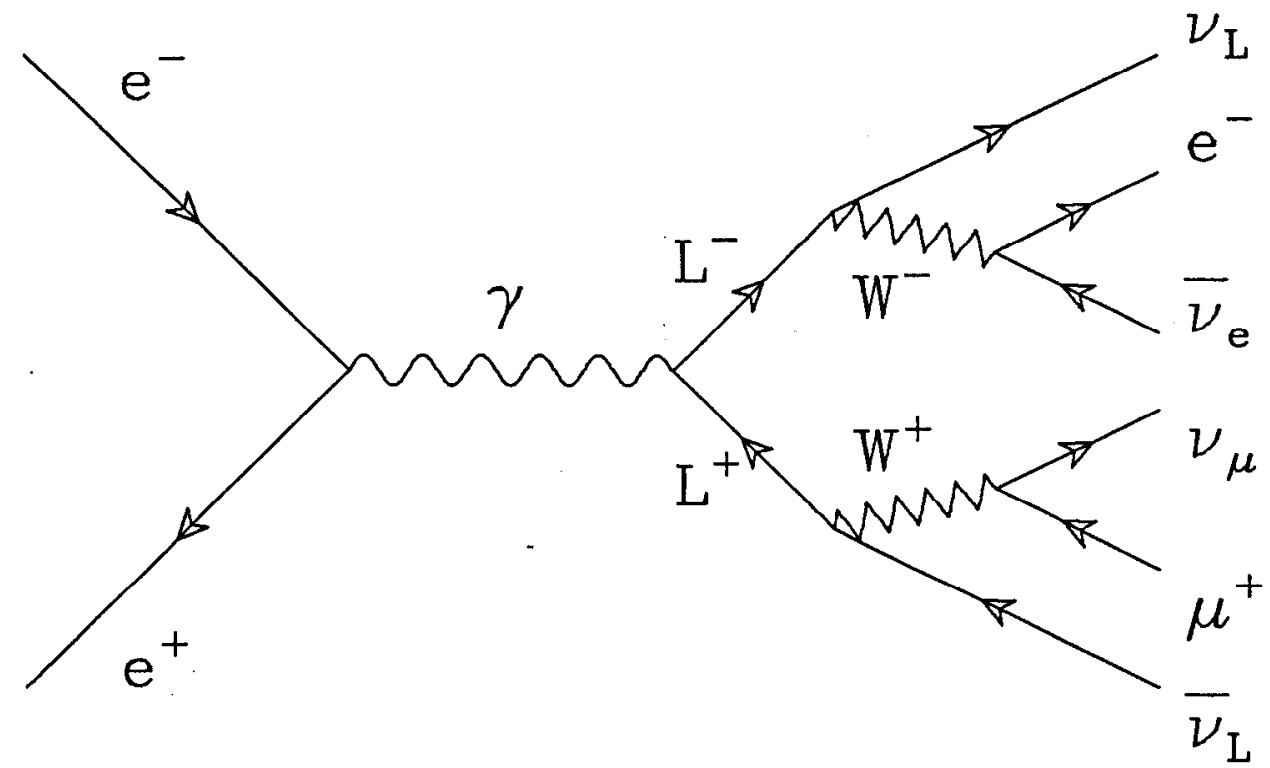

Figure 1.3. Feynman diagram describing the process that is the clearest signal for heavy lepton production. Only the electron and muon are detected. 
The electron-muon tag (and other techniques reliant upon electron or muon identification) become less effective, however, as the neutral lepton mass becomes nearly equal to that of the charged partner. As the neutrino's mass increases, the particle carries away more and more of the available energy, leaving less available to any detectable decay products. In $\mathrm{e}^{+} \mathrm{e}^{-}$annihilation events this leads to two problems touched on earlier.

The first problem is that the background from two-photon processes increases rapidly with lower visible energy. Figure 1.4 shows the total detected charged and neutral energy from a small sample of Mark II data containing only events with two well measured charged particles. The broad peak centered just below the total center of mass energy comes mainly from Bhabha scattering events. Although the Bhabha. cross section is normally considered relatively large, much larger than the cross section for any annihilation process, it is dwarfed by the cross section from two-photon events, occurring at lower detected energies. The peak near zero visible energy would appear even higher than shown in fig. 1.4, if not for poor detection efficiency at the lowest energies.

To understand why the two-photon cross section is so large, it is instructive to consider such processes in general. Figure 1.5 shows the Feynman diagram for a generic two-photon process, leading to the production of some system $\mathrm{X}$ of detected particles, which can consist of hadrons or leptons. The dominant part of the cross section comes from the case where both the incoming electron and positron slightly scatter, each emitting virtual photons of invariant mass squared near zero. The two nearly-real photons coalcscc to form the system X.

The matrix element for this process contains a propagator term for each virtual photon, given for the electron's emitted photon by:

$$
\frac{1}{q^{2}}=\frac{1}{\left(p_{\mathrm{e}}-p_{\mathrm{e}}^{\prime}\right)^{2}}=\frac{1}{2\left(\mathrm{E}_{\mathrm{e}} \mathrm{E}_{\mathrm{e}}^{\prime}-\mathrm{P}_{\mathrm{e}} \mathrm{P}_{\mathrm{e}}^{\prime} \cos \theta+\mathrm{m}_{\mathrm{e}}^{2}\right)}
$$

where $\theta$ is the angle between the incoming and outgoing electron directions, and $\mathrm{m}_{\mathrm{e}}$ 


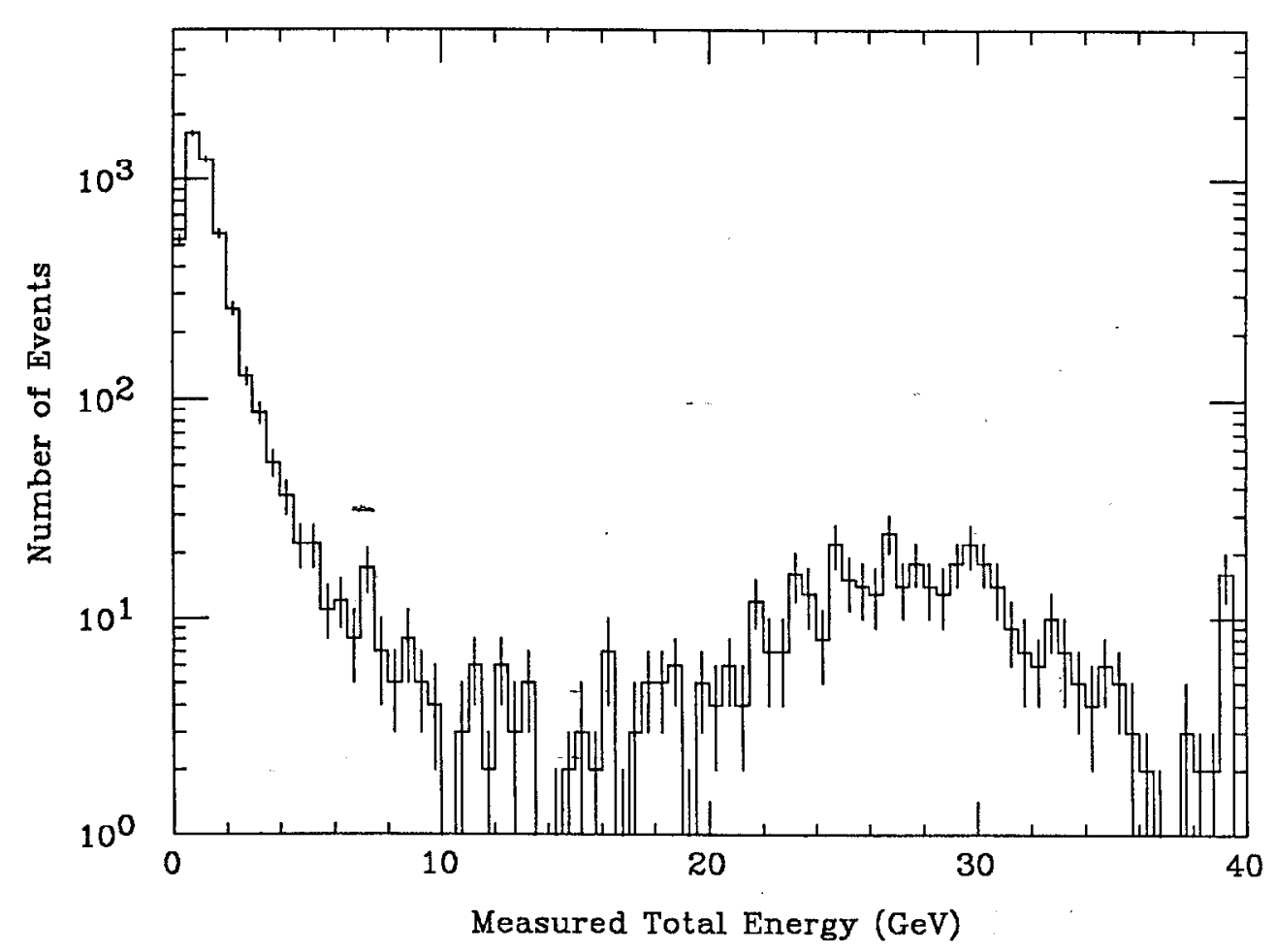

Figure 1.4. Distribution in total measured energy for a small sample of partially filtered events with two detected charged particles.

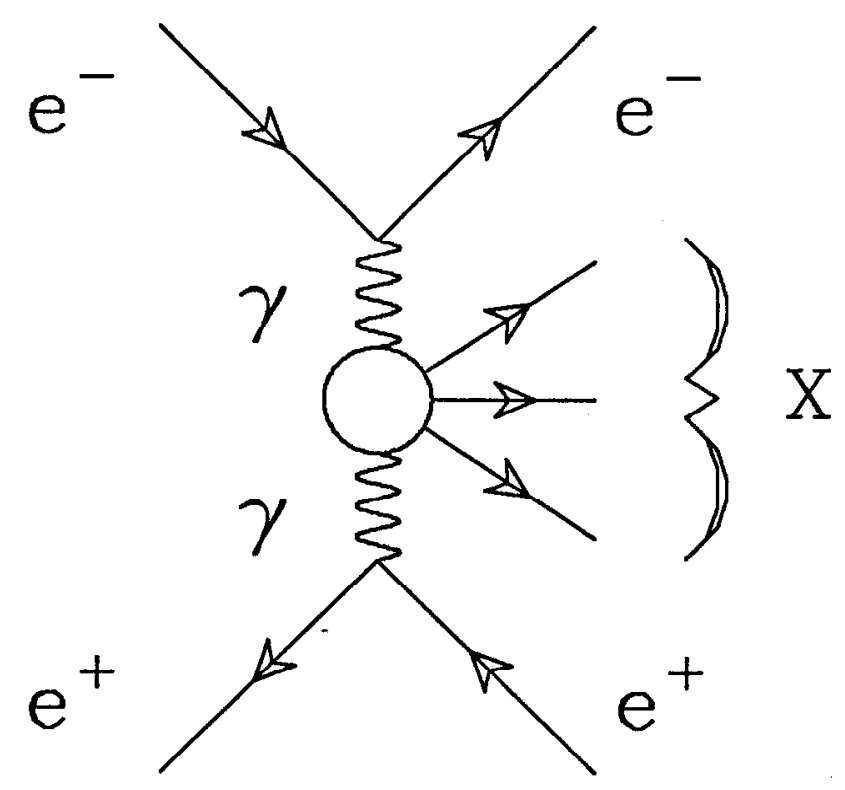

Figure 1.5. Feynman diagram describing generic two-photon production of particles X. For many calculations, the two virtual photons can be considered real, that is, to have zero invariant mass. 
is the electron mass. $p_{\mathrm{e}}$ and $p_{\mathrm{e}}^{\prime}$ are the 4-momenta of the incoming and outgoing electrons, respectively, while $\mathrm{E}_{\mathrm{e}}, \mathrm{E}_{\mathrm{e}}^{\prime}, \mathrm{P}_{\mathrm{e}}$, and $\mathrm{P}_{\mathrm{e}}^{\prime}$ are their energies and momentum magnitudes. For relativistic electron energies $\left(\mathrm{E}_{\mathrm{e}} \approx \mathrm{P}_{\mathrm{e}}, \mathrm{E}_{\mathrm{e}}^{\prime} \approx \mathrm{P}_{\mathrm{e}}^{\prime}\right)$ and for small scattering angles $(\cos \theta \approx 1)$, the propagator can become as large as

$$
\frac{1}{q^{2}} \approx \mathrm{O}\left(\frac{1}{\mathrm{~m}_{\mathrm{e}}^{2}}\right)
$$

Mathematically, it is the largeness of this term that leads to enormous cross sections for two-photon processes.

The second problem with traditional heavy lepton searches at low chargedneutral mass differences is the dependence on electron or muon identification. If identifying electrons relies upon their characteristically large energy deposition in a calorimeter, as is the case for the Mark II detector used in this search, the ability to distinguish electrons from hadrons (primarily pions) is seriously hampered at low energies. For electron energies below $\approx 1 \mathrm{GeV}$, fluctuations in measured electromagnetic shower energy due to the coarseness of the calorimeter and fluctuations in the amount of ionization induced by the passage of real hadrons lead to substantial contamination of electron candidate samples by hadrons. Identifying muons can become even more difficult because lower energy muons cannot travel through enough hadronic interaction lengths of matter to allow distinguishing them from hadrons. For example, in the Mark II detector, the lowest energy allowed a muon candidate is $\approx 600 \mathrm{MeV}$, and at that energy, hadron contamination can be considerable. ${ }^{28}$

Other common techniques for detecting heavy leptons are requirements of large missing energy or large missing momentum transverse to the beam, due to the escape of two or more neutrinos. Unfortunately, for very low charged-neutral mass differences, large missing energy is a characteristic of the dominant background, too, namely two-photon processes where the high energy electron and positron escape the detector at low angles. Requiring missing transverse momentum does significantly 
reduce the two-photon background, but the consequent reduction in signal is unacceptable when expected heavy lepton decay product momenta are less than $\approx 1$ $\mathrm{GeV}$.

\subsubsection{Results of previous searches}

Selected results from previous searches for sequential heavy leptons are shown in fig. 1.6. The region lying to the left and above the dashed line is by definition forbidden. The region below the bottom contour (a) is where a charged lepton would live so long that it would travel on average at least one meter before decaying in $\mathrm{e}^{+} \mathrm{e}^{-}$experiments at TRISTAN $(\sqrt{s}=56 \mathrm{GeV})$. In those experiments, one would expect such a long-lived lepton to have been seen directly. ${ }^{6-8}$ The region bounded by contour b is excluded with $90 \%$ confidence by the UA1 experiment at the CERN Spp̄S collider $^{4}$, but, as noted before, this limit is somewhat model-dependent. The contours labelled $c$ and $d$ define regions excluded with $95 \%$ confidence by the AMY and VENUS experiments at TRISTAN from searches for isolated leptons in coincidence with two jets. Contour $\mathrm{e}$ is an estimate ${ }^{3}$ of the region excluded by PETRA heavy lepton doublet searches that assumed the neutral lepton was massless. Contour $f$ defines approximately the region excluded by the JADE experiment at PETRA in a search for supersymmetric charginos decaying into heavy photinos, ${ }^{29}$ a process that would mimic close-mass lepton decay. The regions bounded by contours $\mathrm{g}$ and $\mathrm{h}$ are excluded* by the Mark II and TPC experiments at PEP, ${ }^{9,10}$ using electron and muon identification techniques. Note that both of these PEP searches and the one described in this work cannot explore the region to the right of the vertical dotted line marking the beam energy of $14.5 \mathrm{GeV}$. The goal of this work is to cxplore the remaining unexcluded

* The region excluded by Mark II is where the probability that the estimated background accounts for the measured data is nine times greater than the probability that the estimated background in addition to a heavy lepton signal accounts for the data. Gaussian error distributions are assumed. The area excluded by TPC corresponds to $99 \%$ confidence level. 


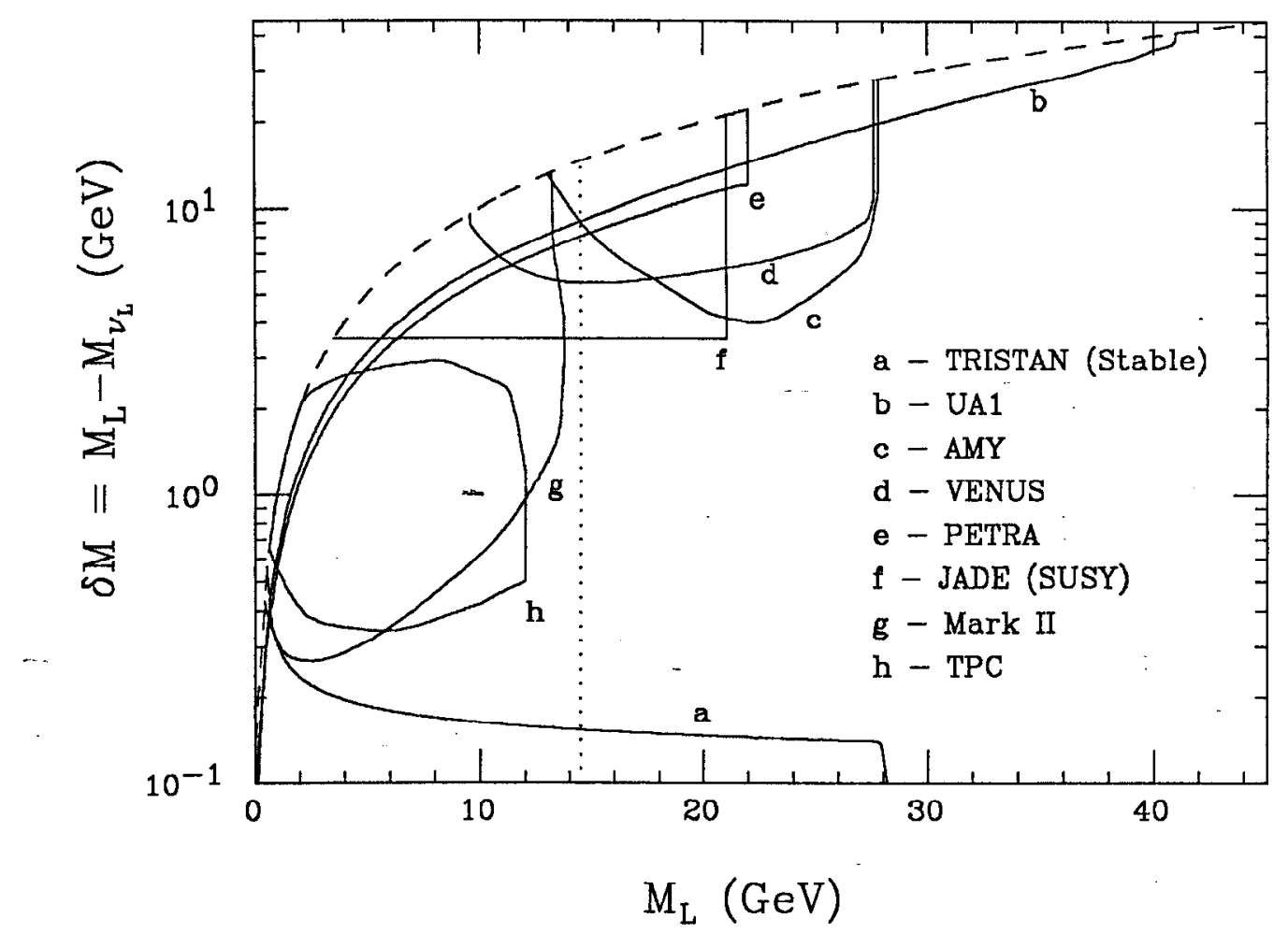

Figure 1.6. The contours bound regions of charged and neutral lepton mass combinations that have been excluded by previous experiments.

region to the left of this line, concentrating upon reaching the lowest accessible mass differences.

\subsubsection{Radiative tagging}

This search uses a new technique to separate the signal for heavy lepton production from the conventional backgrounds when the charged-neutral mass difference is small. The idea is to require detection of an isolated, energetic photon in association with the low energy decay products of the heavy leptons (see fig. 1.7). Although this requirement that radiation be produced during lepton production suppresses the signal by a large factor, it suppresses the dominant background, that from two-photon processes, by a much larger factor.

To understand why this is true, consider fig. 1.8, where a real photon is emitted 


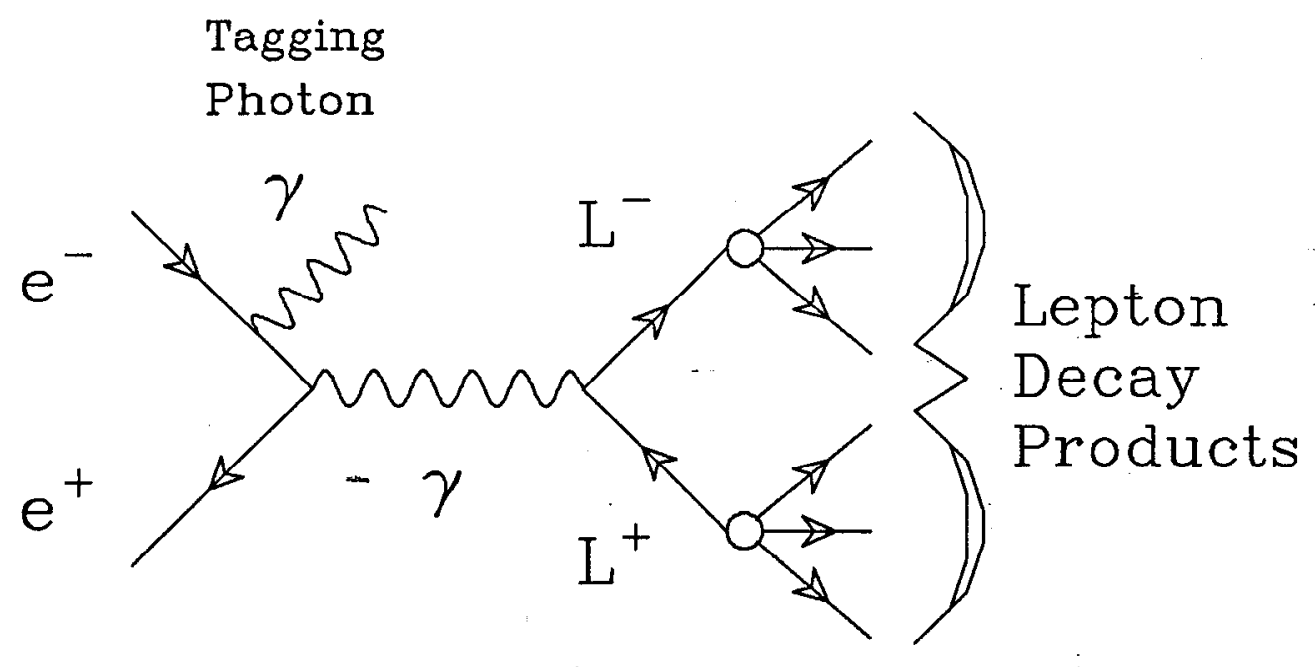

Figure 1.7. Feynman diagram describing radiative production of charged leptons, which decay into various lighter particles.

from the incoming electron. Calculation of the propagator of the virtual photon now gives:

$$
\frac{1}{{q^{\prime}}^{2}}=\frac{1}{\left(p_{\mathrm{e}}-p_{\mathrm{e}}^{\prime}-p_{\gamma}\right)^{2}}=\frac{1}{\left(p_{\mathrm{e}}-p_{\mathrm{e}}^{\prime}\right)^{2}-2\left(p_{\mathrm{e}}-p_{\mathrm{e}}^{\prime}\right) \cdot p_{\gamma}}
$$

Under the same conditions as bcfore, in which $q^{2}$ is minimized, this becomes

$$
\frac{1}{{q^{\prime}}^{2}} \quad \approx \quad \mathrm{O}\left(\frac{1}{\mathrm{E}_{\gamma}^{2}\left(1-\cos \theta_{\gamma}\right)}\right)
$$

where $\theta_{\gamma}$ is the angle between the detected photon and the beam. For photons of energy $>1 \mathrm{GeV}$ detected in the Mark II central calorimeter $\left(\cos \theta_{\gamma} \leq 0.7\right)$, the ratio of the virtual photon propagators in radiative vs non-radiative two photon processes is $\approx 10^{-6}$. Since the ratio of the radiative to the non-radiative cross sections will depend on the square of this propagator ratio, it is clear that one can hope for a huge suppression of the two-photon backgrounds with a radiative tag.

A number of caveats should be mentioned at this point. One is that there are other processes of the same order in $\alpha_{\mathrm{QED}}$ as the two photon background considered here. For example, fig. 1.9 shows diagrams necessary to describe to lowest order the 


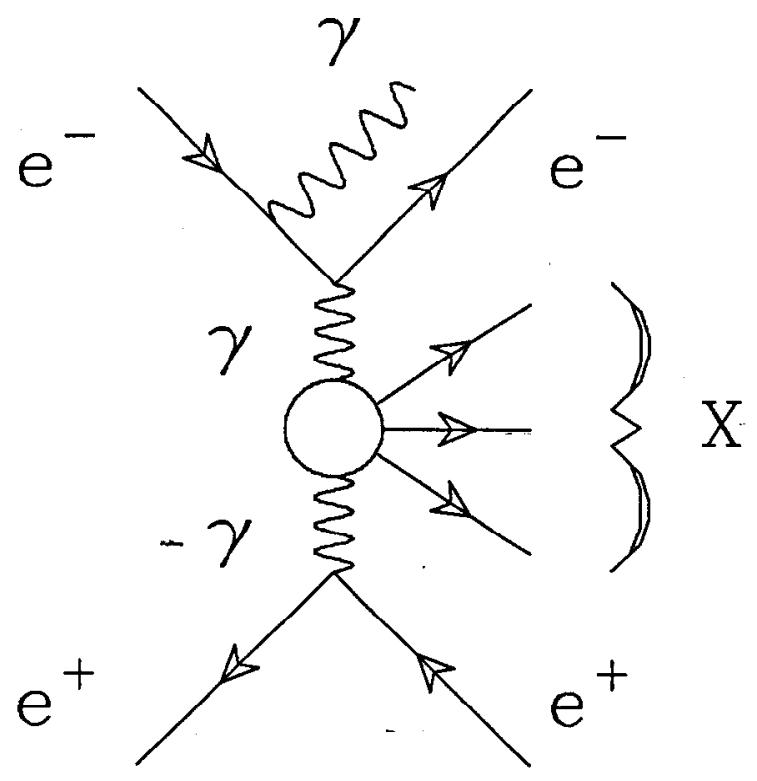

Figure 1.8. Feynman diagram describing two-photon process accompanied by initial state radiation.

process $\mathrm{e}^{+} \mathrm{e}^{-} \rightarrow \mathrm{e}^{+} \mathrm{e}^{-} \mu^{+} \mu^{-}$. Normally, the first diagram completely dominates when both the electron and positron escape at low angles, but the addition of radiation to the diagrams, suppresses the first process so much that the others become relatively important. These additional diagrams provide a measurable background, but one that can be easily suppressed by requiring the detected photon be isolated from any charged tracks in the event. Requiring a large missing transverse momentum of the event, taking into account the tagging photon, further suppresses this background.

Another caveat to keep in mind is that photons occur in the decays of neutral pions $\left(\pi^{0} \rightarrow \gamma \gamma\right)$. This means that two-photon backgrounds involving direct production of neutral pions or indirection production through charged rho particles, can lead to energetic, isolated photons in the central detector. Again, however, these backgrounds can be largely suppressed by requiring the missing transverse momentum be substantial.

In addition, there is the problem of radiative tau pair production as a background. Unfortunately, for very heavy leptons, the requirement of radiative production reduces the signal/background ratio with respect to tau pair production because the available 


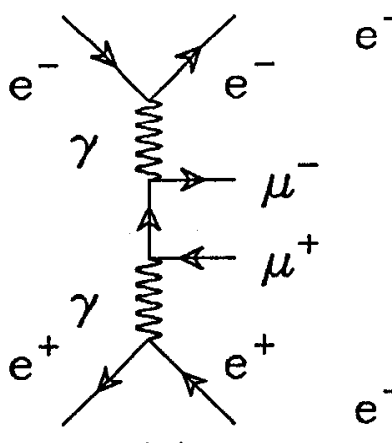

(2)

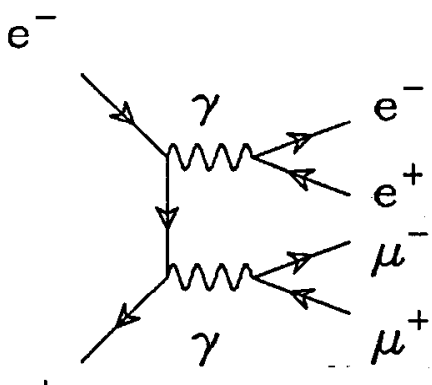

(2)

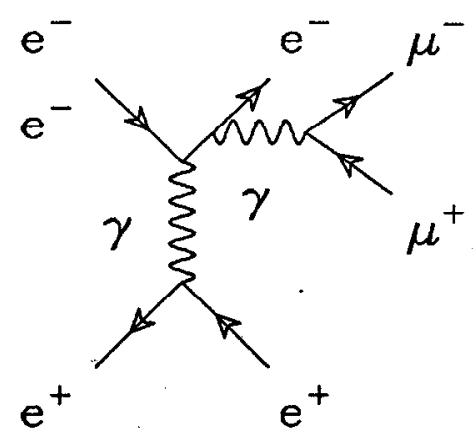

(4)

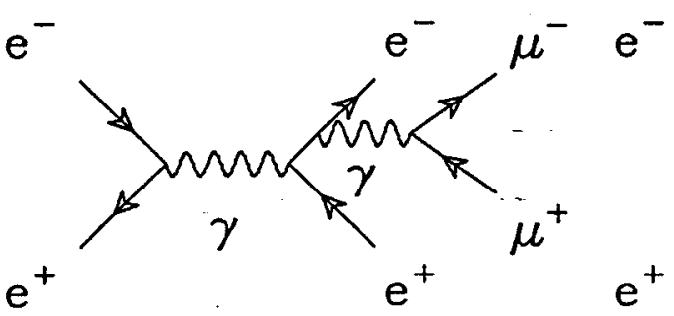

(2)

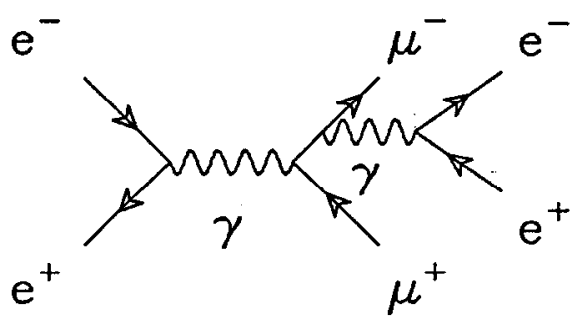

(2)

Figure 1.9. Feynman diagrams describing various processes leading to production of an electron and muon pair. There are 7 additional diagrams that are permutations of these 5. Normally, the first of these five and its permutation dominate in the cross section, but adding detectable radiation suppresses the first diagram's contribution enormously. The numbers in parentheses indicate the total number of permutations of each type of diagram.

phase space for production of the leptons rapidly becomes small as the photon energy increases. When an electron or positron emits a photon, it collides with its antiparticle in a reference frame with energy reduced from the original $29 \mathrm{GeV}$. As that center-ofmass energy is reduced to a value near twice the lepton mass energy, the production of a lepton-antilepton pair is suppressed by a factor

$$
\frac{\beta\left(3-\beta^{2}\right)}{2}
$$

where $\beta$ is the velocity (in units of $c$ ) of the outgoing lepton in the c.m. frame. For a given photon energy, this factor can be much smaller for a heavy lepton than for a 
tau particle. In fact, as will be discussed in chapter 3, kinematics forbid production of high energy photons in association with very heavy leptons. Consequently, very heavy leptons $\left(\mathrm{M}_{\mathrm{L}}>10 \mathrm{GeV}\right)$ will be difficult to extract from the tau background.

One final caveat, which will be discussed in more detail in the next chapter, is that for very low charged-neutral lepton mass differences, the available phase space for the charged lepton's decay becomes quite small. If this leads to a total decay width small enough that the lepton lives long enough to travel a significant distance in the detector before decaying, then charged particle detection, in particular, triggering of the detector readout, becomes difficult. Ultimately, this lifetime consideration, more than any other, limits one's ability to search for very low mass difference lepton doublets with the Mark II detector. As will be discussed in the final chapter, this limitation due to the Mark II trigger need not apply to future experiments or even to other existing data sets taken at the PEP or PETRA storage rings.

\subsection{Outline of Thesis}

Chapter 2 discusses the important properties of heavy lepton production and decay, derived largely from studies of events generated by the LULEPT Monte Carlo program, which is described in detail in Appendix A. Chapter 3 considers details of radiative production of fermion-antifermion pairs, including the importance of final state radiation in the signal and in radiative tau pair production. Chapter 4 describes briefly the PEP storage ring and, in some detail, the Mark II detector, with emphasis on those elements important to this search. Chapter 5 describes the experimental analysis, presents results from the heavy lepton search, and discusses the prospects for improving the sensitivity of future lepton searches. 


\section{Chapter 2}

\section{Close-Mass Lepton Properties}

This chapter describes briefly our current understanding of weak interactions and how that understanding allows one to calculate with confidence the properties of a fourth generation close-mass doublet. In particular, the expected decay modes of the charged lepton are discussed, including the total decay rates. In the case of very small mass differences, the total decay rate can become small enough that the charged lepton's finite lifetime makes detection of its decay products quite difficult.

\subsection{Weak Interactions}

The study of weak interactions began with the discovery of nuclear $\beta$ decay just before the turn of the century. ${ }^{30}$ The first peculiarity noticed about the interaction was that it appeared to violate both momentum and energy conservation. For example, when a neutron decays into a proton by emission of an electron, the total energy of the proton and the electron does not match what one would expect from kinematics. Wolfgang Pauli suggested that in nuclear $\beta$ decay another particle was being emitted that somehow escaped detection. ${ }^{31}$ Enrico Fermi followed up on this idea, proposing not only a new particle, which he called the neutrino, but also outlining a theory of $\beta$ decay that was remarkably prescient. ${ }^{32}$ This theory is somewhat contrived, however, lacking the simplicity of the later, immensely successful theory of quantum electrodynamics.

The first failing of the Fermi model is its postulate of so-called "four-fermion" 
interactions, in which four elementary particles interact at a point. One example is shown in fig. 2.1, where an incoming neutrino scatters elastically from an electron. The cross section for this interaction can be calculated from the Fermi theory: ${ }^{30}$

$$
\sigma=\frac{4 \mathrm{G}_{\mathrm{F}}^{2}}{\pi} \mathrm{E}_{\nu}^{2}
$$

where $E_{\nu}$ is the neutrino energy in the center-of-mass frame and $G_{F}$ is the Fermi coupling constant, which has dimension of inverse mass squared. Unfortunately, as the neutrino energy increases, this cross section becomes so large as to become unphysical. Such large cross sections are not a problem at lower energies because of the low value of $\mathrm{G}_{\mathrm{F}}\left(=1.17 \times 10^{-5} \mathrm{GeV}^{-2}\right)$. This suggests that the four-fermion interaction of fig. 2.1 is really two three-fermion interactions, linked by a massive intermediary particle $\mathrm{W}$, as shown in fig. 2.2. The factor of $\mathrm{G}_{\mathrm{F}}^{2} / 2$ is then replaced by $\mathrm{g}^{4}$ times the square of the propagator term for the intermediate $\mathrm{W}$, where $\mathrm{g}$, analogous to electric charge, is a dimensionless coupling constant describing the interaction strength at each three-fermion vertex. The propagator term is then (neglecting the momentum transfer relative to the $\mathrm{W}$ mass) $1 / \mathrm{M}_{\mathrm{W}}^{2}$. If one makes the identification

$$
\frac{\mathrm{G}_{\mathrm{F}}}{\sqrt{2}} \rightarrow \frac{\mathrm{g}^{2}}{\mathrm{M}_{\mathrm{W}}^{2}},
$$

and assumes that $\mathrm{g}$ is of the same order as the electromagnetic coupling e, then one deduces a $\mathrm{W}$ mass of about $100 \mathrm{GeV}$.

That there might exist such a heavy intermediary particle was suspected for many years, but accelerators did not possess the necessary energies to explore the possibility directly. Various experiments established that charged current interactions, such as that in fig. 2.2, where the intermediary particle possesses electric charge, are dominated by vector dynamics. In other words, if such an intermediary particle existed, it must have a spin of 1 . With the suggestion and subsequent discovery of parity violation in weak interactions, ${ }^{33,34}$ numerous experiments established that, in fact, 


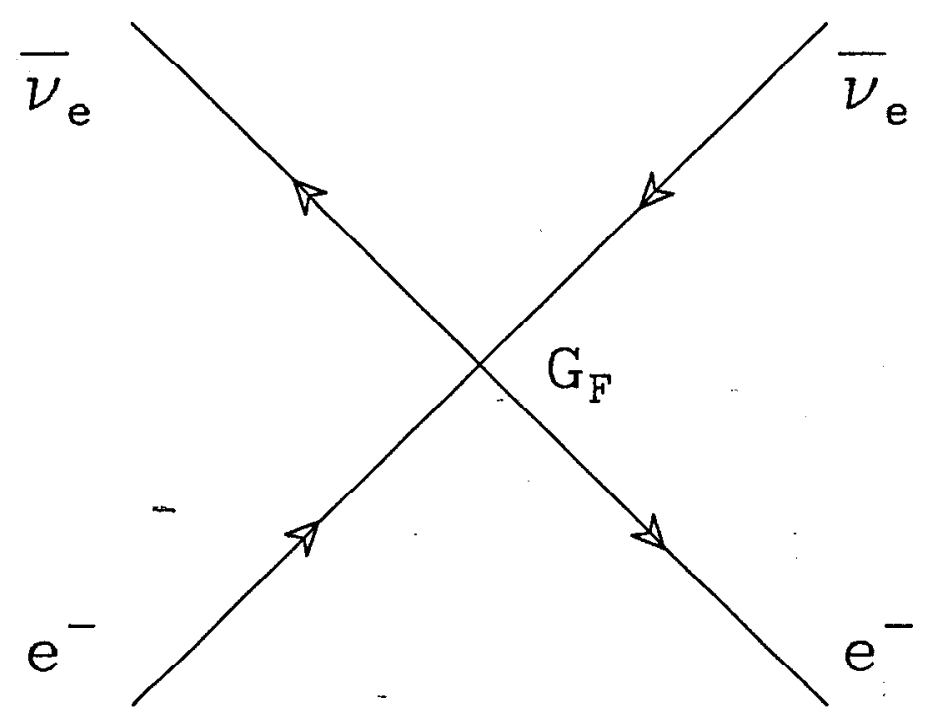

Figure 2.1. Feynman diagràm for four-fermion weak interaction, where the presence of an intermediary particle is parameterized away with the Fermi coupling constant $\mathrm{G}_{\mathrm{F}}$.

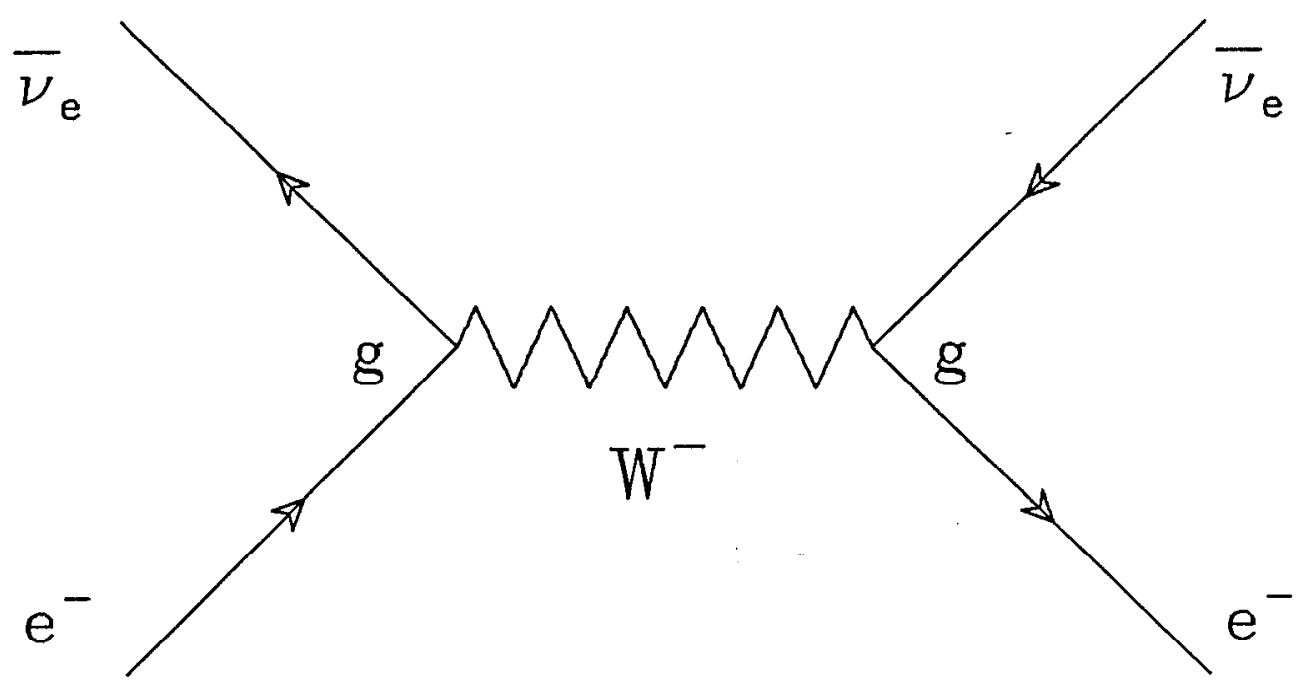

Figure 2.2. Modern Feynman diagram for weak interaction, where the presence of the intermediary particle is indicated explicitly.

both vector and axial vector amplitudes are involved in roughly equal proportions. For processes involving only leptons, it was suggested ${ }^{35}$ that one could draw a strong 
analogy between electrodynamics and weak interactions by making the substitution:

$$
\frac{\mathrm{e}^{2}}{q^{2}} \psi^{\dagger} \gamma^{\mu} \psi \quad \rightarrow \quad \frac{\mathrm{g}^{2}}{q^{2}-\mathrm{M}_{\mathrm{W}}^{2}} \psi^{\dagger}\left(1-\gamma^{5}\right) \gamma^{\mu} \psi
$$

where $\psi$ and $\psi^{\dagger}$ describe the wave functions of the fermions interacting with the $\mathrm{W}$, and $\gamma^{\mu}, \gamma^{5}$ are the usual Dirac matrices. ${ }^{36} \mathrm{~A}$ slightly more complicated substitution describes hadronic weak interactions with some accuracy.

Numerically, this technique works fairly well, giving experimentally verified predictions, but the strict analogy with electrodynamics is ultimately unsatisfactory. The problem is that the theory of quantum electrodynamics can be renormalized only because the photon is massless. Renormalization means that when one calculates higher order terms of a perturbation expansion in powers of the dimensionless coupling constant, that one obtains sensible results, that is, finite answers. With a massive intermediate boson, these higher order terms diverge. One can make lowest order predictions for various cross sections or decay rates with reasonable accuracy, but when one attempts to calculate higher order corrections, one gets nonsense.

The solution to this dilemma turns out, it is believed now, to reside in a phenomenon called spontaneous symmetry breaking. ${ }^{37,38}$ One hypothesizes that in some sense the "true" mass of the W particle is indeed zero, allowing the theory to be renormalized, and thereby allowing sensible higher order calculations. But one also postulates a new particle field, called the Higgs, which through its interactions with other particles, imparts mass to them. Although in many ways also quite contrived, this theory provides a direct unification of the electromagnetic and weak interactions and has as yet no known internal inconsistencies. In addition, the theory predicts that the charged $\mathrm{W}$ particle has a neutral partner of slightly greater mass, called the $\mathrm{Z}$ vector boson, which is responsible for neutral weak interactions. The existence of these $\mathrm{W}$ and $\mathrm{Z}$ bosons was beautifully confirmed in 1983 in collisions between protons and anti-protons at center-of-mass energies of $540-550 \mathrm{GeV} \cdot{ }^{39,40}$ 
In summary, although the history of our understanding of weak interactions is replete with confusion and theoretical contrivances, there have also been many remarkably insightful guesses that have led finally to a theory that is sufficiently accurate and, with the notable exception of the Higgs mechanism, satisfyingly simple. It is the success of the unified electroweak theory that gives one confidence in predicting the properties and interactions of an unseen-close-mass lepton doublet.

\subsection{Calculation of Heavy Charged Lepton Decay Widths}

Explicit formulas for differential and integrated partial widths of the decay modes - discussed in this section can be found in the appendix. Given here is an overview of those calculations, in particular, the various assumptions that have been made.

When the heavy charged lepton decays into its neutrino partner and other particles, the actual production of the other particles is through a virtual charged W boson, as shown in fig. 2.3. How the $\mathrm{W}$ particle materializes into the detected particles varies considerably, depending upon the nature of the decay products.

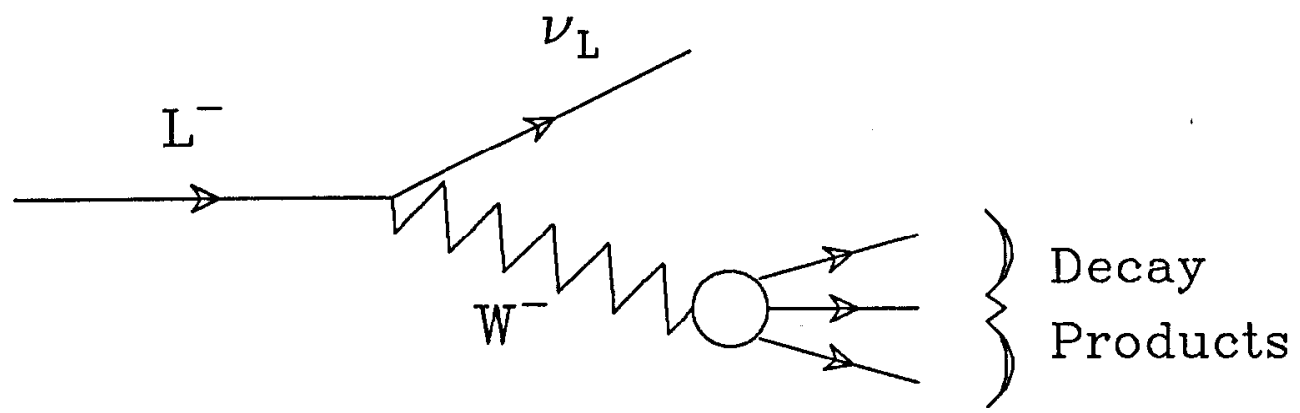

Figure 2.3. Feynman diagram for general decay of a charged lepton into its neutrino partner and a virtual $\mathrm{W}$ boson, which materializes as conventional leptons or hadrons.

In the simplest case, the $W$ materializes as another, lower-mass, lepton doublet, for example, $\mathrm{W} \rightarrow \mathrm{e} \overline{\nu_{e}}$. In this case, the electroweak theory predicts an unambiguous rate, based on measured values of the electroweak coupling constants. The validity 
of the calculations is confirmed by the measured values of the partial decay widths for $\mu \rightarrow \nu_{\mu} e \bar{\nu}_{e}, \tau \rightarrow \nu_{\tau} e \bar{\nu}_{e}$, and $\tau \rightarrow \nu_{\tau} \mu \bar{\nu}_{\mu}$, although the exact agreement for the tau decays is still somewhat controversial, depending upon measured values of the tau lifetime. ${ }^{41}$

For this analysis, it has been assumed that the coupling of the $\mathrm{W}$ to the closemass lepton doublet is the same as for known lepton doublets, that is, V-A. Note, however, that since the heavy neutrino partner has a non-zero mass, its helicity is not invariant. Unlike that of a massless neutrino, its direction of spin depends upon one's reference frame. Thus the conventional designation of neutrinos as strictly "lefthanded" is not appropriate here. Note also that the operator $1-\gamma^{5}$, used in the decay calculations, does permit appreciable couplings to "right-handed" fermions when the fermion mass is substantial, relative to the energy scale of the interaction.

When the $\mathrm{W}$ materializes as hadrons, the situation becomes more complicated. From the viewpoint of fundamental particles, the $\mathrm{W}$ materializes as a quark-antiquark doublet, for example, as a down and an anti-up quark pair. Then one imagines the quark and anti-quark joining to form a single hadron, such as a charged pion, or in another extreme, flying apart and forming through "hadronization" many pions or other hadrons. As mentioned in the first chapter, this process of hadronization is not well-understood, but has been described with some success by phenomenological models.

One expects from the theory of quantum chromodynamics that simulation of hadronization is more reliable when the relative momentum of the quark and antiquark is large, so that non-perturbative effects are less dominant. At lower momenta, one cannot expect hadronization modelling to give accurate results. Instead, it is preferable to relate the heavy lepton decays to other, directly measured processes.

As a simple example, the materialization of a charged $W$ into a single charged pion, however many intermediate quarks and gluons are involved, can be related to 
the inverse process of a charged pion transforming into a $\mathrm{W}$ in the observed reaction $\pi^{-} \rightarrow \mathrm{W}^{-} \rightarrow \mu^{-} \bar{\nu}_{\mu}$. Another example is use of the conserved vector current theorem, which relates the cross section for $\mathrm{e}^{+} \mathrm{e}^{-} \rightarrow \mathrm{X}$ to the production of $\mathrm{X}$ through a W, where $\mathrm{X}$ has spin 1 . This theorem proves particularly useful in calculating heavy lepton decays to rho particles. More detail on these two examples is given in the appendix.

For this analysis, several exclusive hadronic modes are explicitly simulated with such techniques. The validity of these various calculations has been checked by applying them to known tau decay modes and comparing predicted values with those observed experimentally. In general, agreement is very good, giving one confidence in extrapolating the techniques to higher masses. For large mass differences, above $1 \mathrm{GeV}$, the hadronization schemes of the LUND Monte Carlo program ${ }^{42}$ are used to simulate remaining hadronic decay modes not already explicitly simulated. Again, observed tau particle decay modes allow a cross check, and in this instance, provide normalization of otherwise arbitrary decay rates.

An additional consideration in simulating heavy lepton decays is spin-spin correlation. The $\mathrm{V}-\mathrm{A}$ coupling of fermions to the intermediate $\mathrm{W}$ particle leads to a correlation between the momentum directions of the decay products in the heavy lepton's rest frame and the direction of the heavy lepton's spin. This angular correlation in the heavy lepton frame then produces a correlation between the lab frame decay product energies and the angle between the lepton's spin and its momentum in the lab frame. Since the spin direction is as likely to be parallel as anti-parallel to the lepton momentum, the energy distributions for events where the lepton's spin is unknown are unaffected by the correlation. But the spins of the heavy lepton and anti-lepton are also correlated, which implies there remains a correlation between decay product energies for the heavy lepton and anti-lepton. This correlation is properly simulated, as described in the appendix, but for this analysis, its effect is hardly measurable.

Figure 2.4, taken from the appendix, shows the branching ratios for various 
decay modes simulated by the Monte Carlo, plotted vs the lepton mass difference for a charged lepton mass of $10 \mathrm{GeV}$. These branching ratios are relatively insensitive to the charged lepton mass, but extremely sensitive to the mass splitting, as indicated by the rapid variations in the figure.

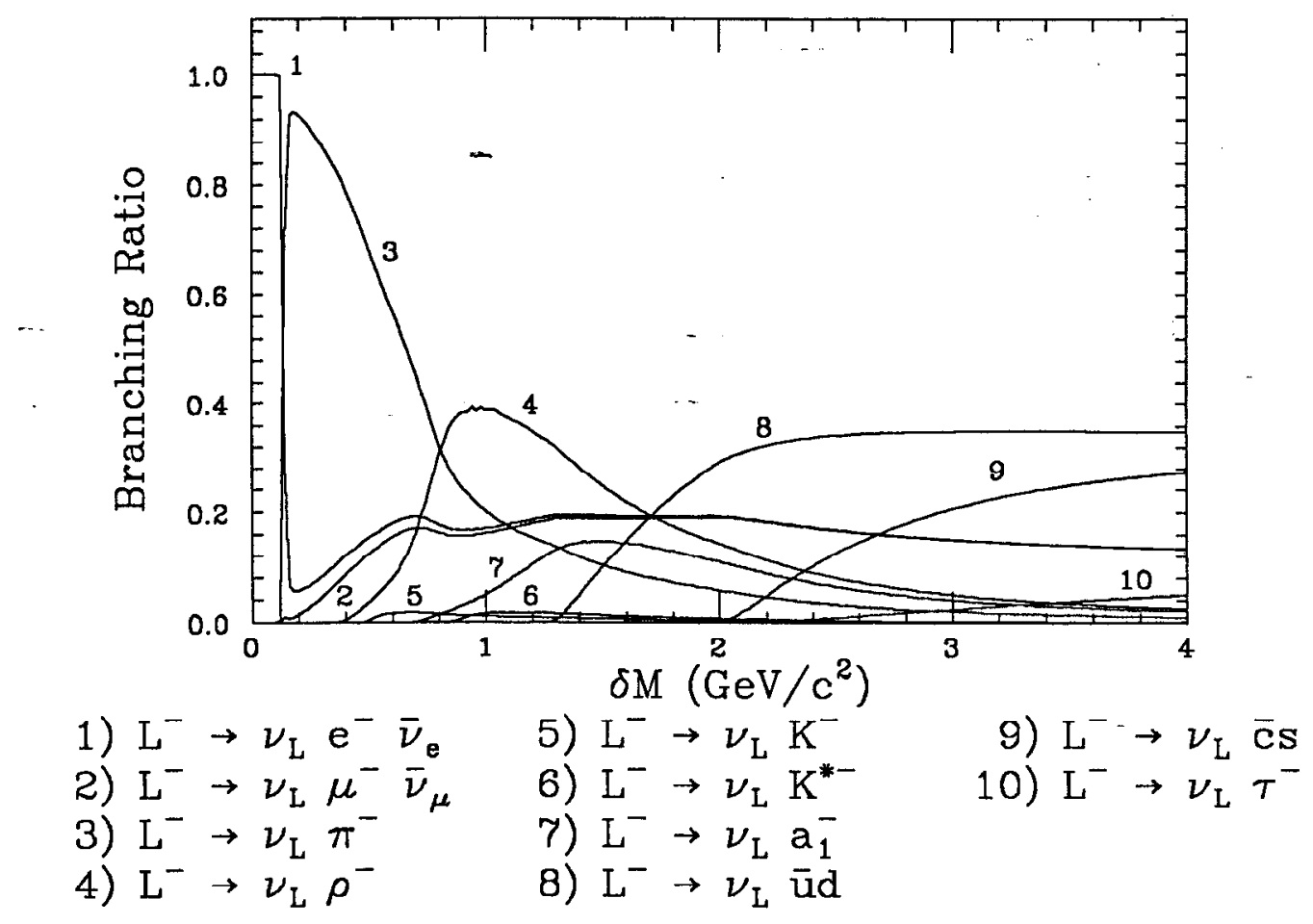

Figure 2.4. Plotted are calculated branching ratios for various heavy lepton decay modes vs the charged-neutral mass difference. The charged lepton mass is fixed at $10 \mathrm{GeV}$.

These rapid variations dictate to some degree the optimal search strategy for exploring diffcrent regions of mass difference. At the very lowest differences, bclow the charged pion threshhold, the decay mode to an electron completely dominates. As mentioned in the first chapter, the decay rate in this region is so low that searches for heavy stable charged particles have already excluded this possibility up to very large charged lepton masses.

Just above the charged pion threshhold, however, the pion mode rapidly over- 
takes and swamps the electron and muon modes. In this region, electron and muon identification are not especially helpful in extracting a heavy lepton signal. For higher mass differences, the leptonic modes gradually become more important and stabilize at about $30 \%$. For mass differences above several hundred $\mathrm{MeV}$, the rho decay mode becomes important, and for higher differences, the $a_{1}$ mode begins to come in. Ultimately, hadronic jets come to dominate the-decay modes.

Since the primary aim of this analysis is to explore very low mass differences, the pion decay mode is the most relevant. That this limits the usefulness of electron and muon identification is moot, since the associated low energies of these decay products preclude such identification with the Mark II detector anyway. As will be described in the final chapter, events are selected that contain two low-energy charged particles, in addition to the tagging photon. No selection cuts are based on electron or muon identification criteria, although the final candidates from the data are checked statistically for consistency with the number of electron candidates expected from background.

\subsection{Lifetime Considerations}

For very low mass differences, the lifetime of the charged lepton becomes substantial, which means the lepton can travel a large distance in the detector before decaying. This can lead to decay product trajectories that do not extrapolate back to the beam collision point. As will be discussed in chapter 4 , large impact parameters can degrade detector trigger efficiency significantly. To avoid uncertainties in charged track trigger Monte Carlo simulation, no such simulation is performed in this analysis. Instead, efficiency corrections for unsimulated effects are applied, based upon direct measurements from the data.

Specifically, the dependence of the trigger efficiency upon the particle's impact parameter is studied with $K_{\mathbf{s}}^{0}$ decays in two-photon events. Unfortunately, the number of such decays producing a charged pion with an impact parameter greater than $5 \mathrm{~cm}$ 
is statistically inadequate. Therefore, to be conservative, a requirement is imposed in this analysis that both detected charged particles have an impact parameter less than $5 \mathrm{~cm}$, since the trigger efficiency for larger-impact-parameter particles cannot be measured directly with confidence.

To see how this affects overall detection efficiency, one can look first at the average distance the charged lepton travels before decaying, as shown in fig. 2.5. This average distance is plotted vs the mass difference for various values of the charged lepton mass, where the charged lepton is assigned the full beam energy of $14.5 \mathrm{GeV}$. The sudden drop that occurs around $140 \mathrm{MeV}$ for each curve corresponds to the opening up of the charged pion decay mode, which sharply increases the decay rate, thus shortening the lifetime. For a particular mass difference, lighter charged leptons travel a longer. distance before decaying because of their larger Lorentz time dilation factor.

In general, the impact parameter of the decay products will scale with the distance $\mathrm{d}$ from the beam collision point to the decay point. As shown in fig. 2.6. the impact parameter is $\mathrm{I}_{\mathrm{P}}=\mathrm{d} \sin \theta$, where $\theta$ is the angle between the decay product's momentum and the original heavy lepton's momentum. There is a correlation, however, between the charged lepton mass and the distribution in $\theta$, because the greater Lorentz boosts associated with lighter charged leptons tend to yield decay products with small $\theta$, that is nearly parallel to the boost axis. This effect is shown in fig. 2.7, where the root-mean-square of $\sin \theta$ is plotted vs the mass difference for the same values of charged lepton mass shown in fig. 2.5. Only the distribution for the simple two-body pion decay mode is shown, since it dominates in the region where impact parameters are large. Comparing figures 2.5 and 2.7, one might guess that the impact parameter does not depend strongly upon the charged lepton mass. A reasonable hypothesis is that the boost factor that makes light leptons travel farther is cancelled by the factor that makes $\sin \theta$ small for their decay products. This approximation is, in fact, a fairly good one, although it breaks down badly for very heavy leptons where the impact parameter must approach zero, regardless of mass difference, as the 


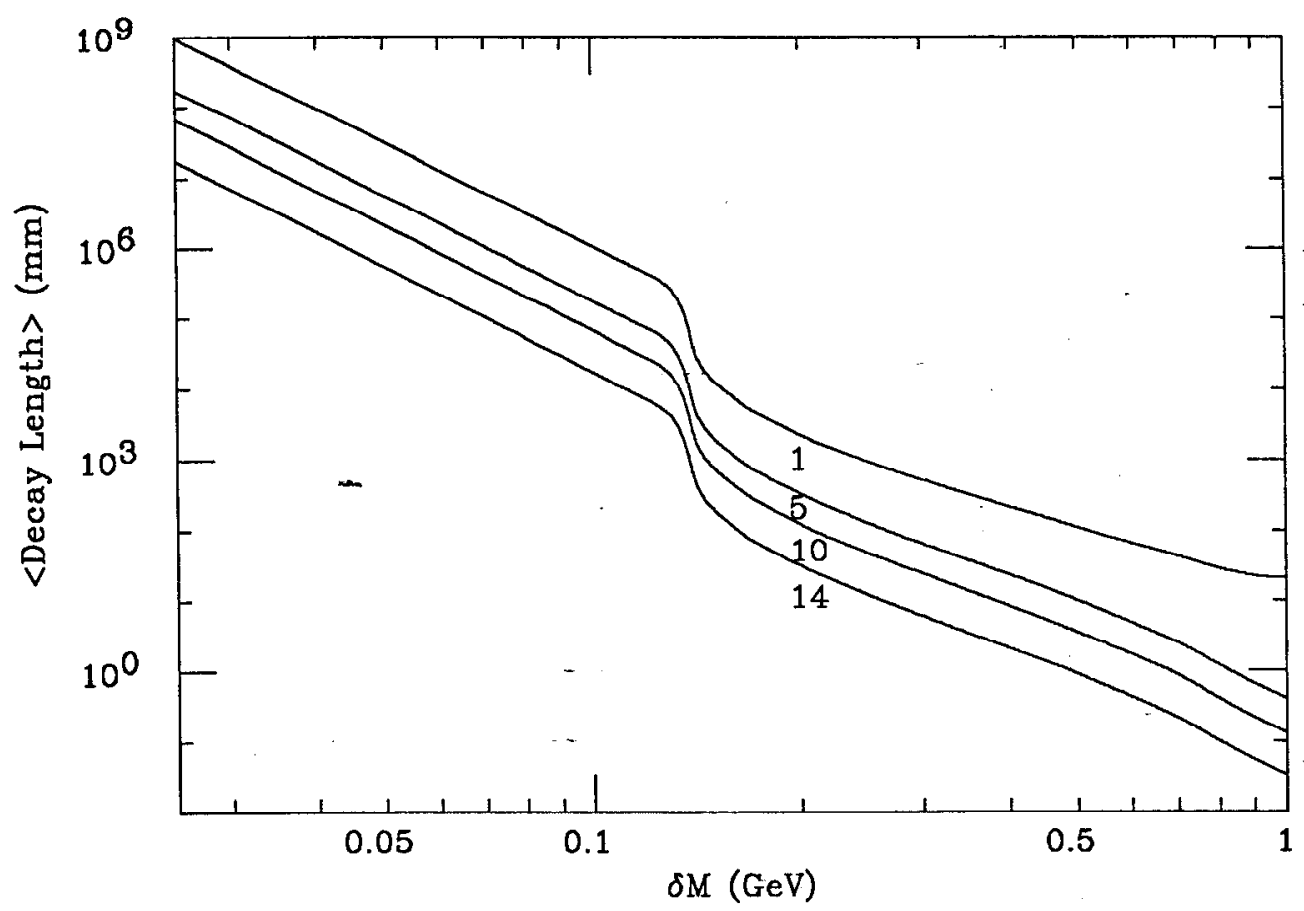

Figure 2.5. Plotted is the average distance travelled by the heavy charged lepton before decaying vs the mass difference between the charged and neutral partners. Curves are shown for charged lepton mass values of $1,5,10$, and $14 \mathrm{Gev}$.

distance the lepton travels before decay approaches zero.

Figure 2.8 shows the impact parameter, calculated from the product of the values in figures 2.5 and 2.7 , plotted vs the mass difference for the same values of charged lepton mass. As expected, the impact parameter does not depend as strongly upon the charged mass, but it does depend sensitively upon the mass difference. Also shown is a horizontal line corresponding to the $5 \mathrm{~cm}$ cut imposed in this analysis. (Strictly speaking, the cut is based on the impact parameter in the plane transverse to the beam.) From the figure, it is clear that exploring the region of mass difference below $\approx 250 \mathrm{MeV}$ is quite difficult.

These arguments concerning impact parameter are somewhat over-simplified in that they do not include the effects of substantial initial state radiation, which reduces 


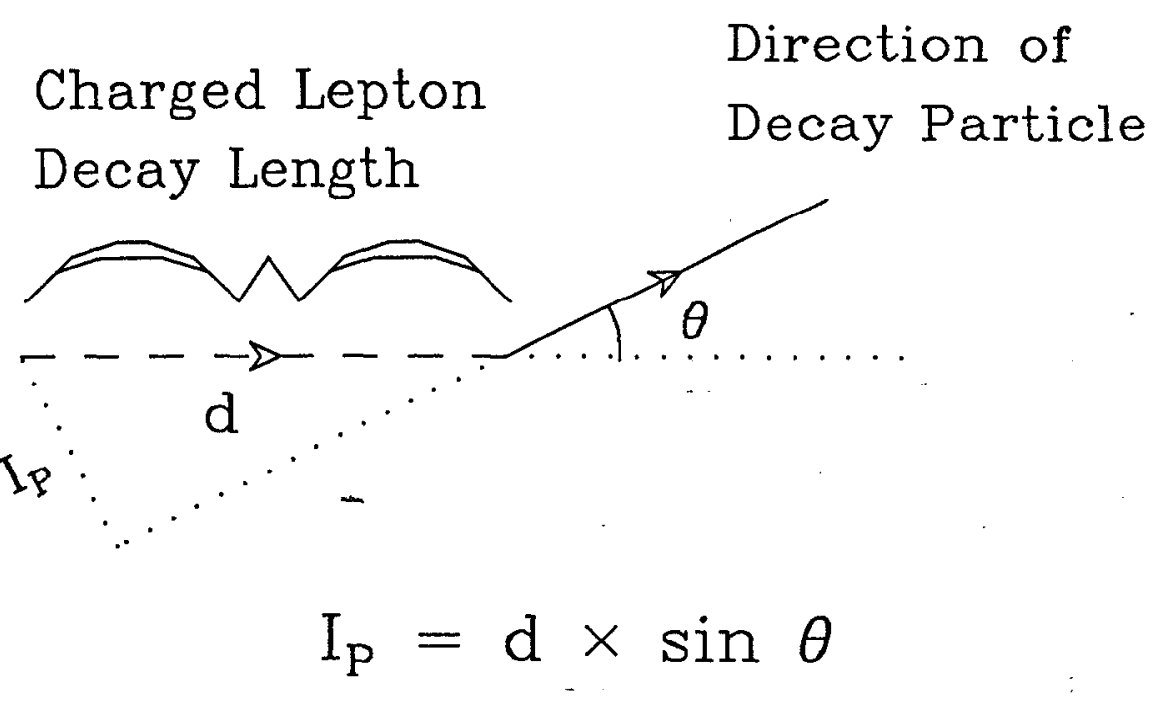

Figure 2.6. The impact parameter of the lepton decay product is defined can be expressed in terms of the heavy lepton decay length and the angle between the momentum directions of the heavy lepton and its decay product.

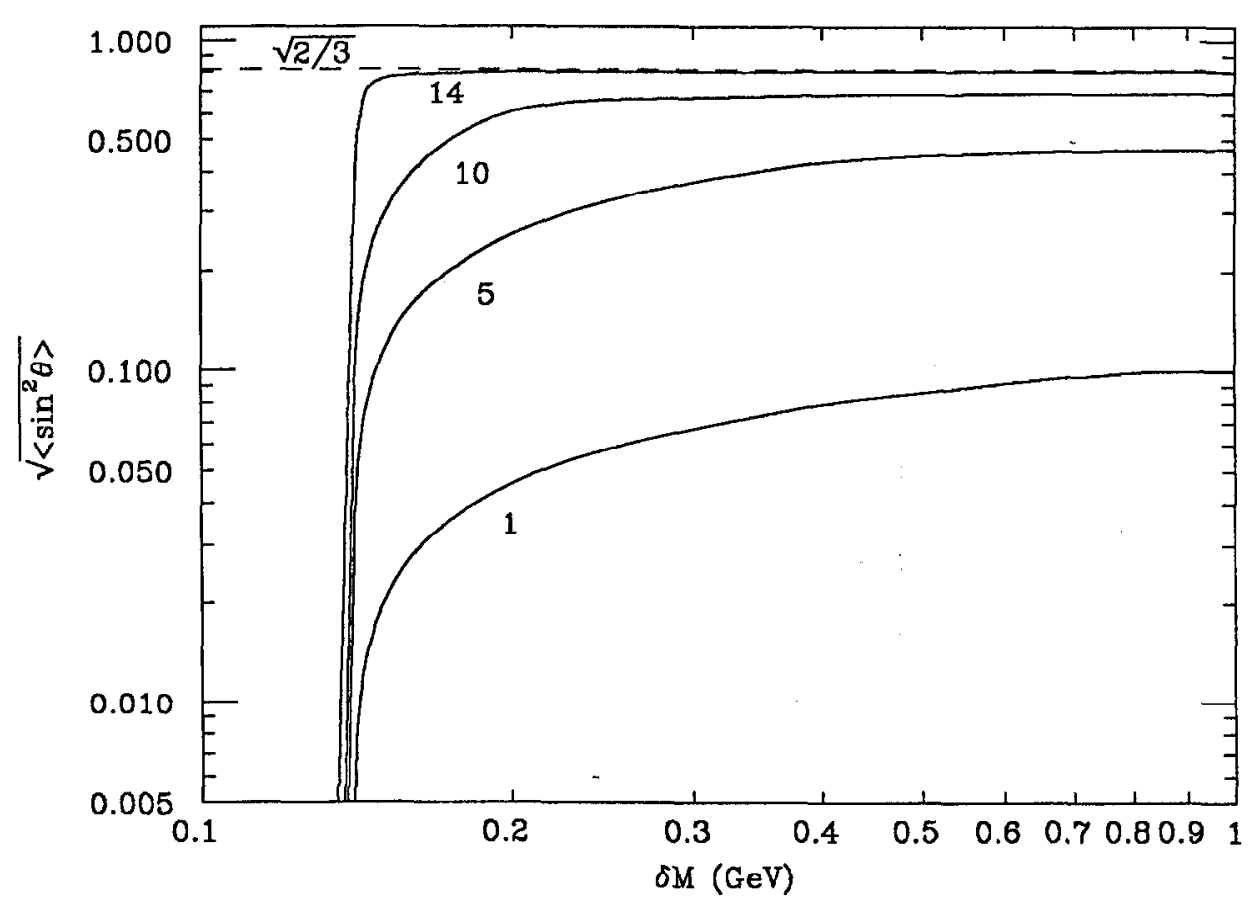

Figure 2.7. Plotted vs mass difference is the root-mean-square of $\sin \theta$, where theta is the angle between the heavy charged lepton and its decay product directions. 


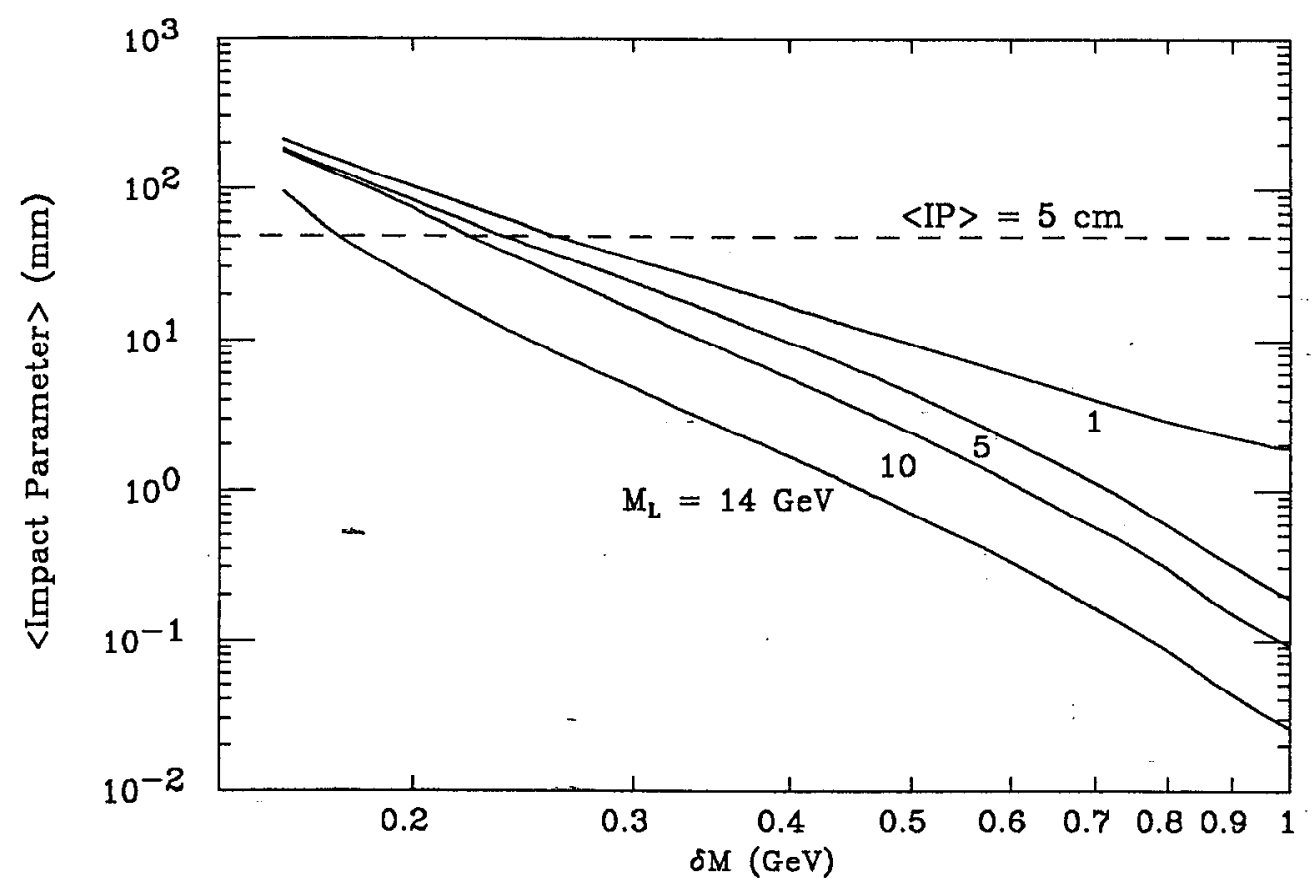

Figure 2.8. Plotted vs mass difference is the average impact parameter of a decay product charged pion, defined as the average heavy lepton decay length multiplied by the root-mean-square of $\sin \theta$ between the charged lepton and pion directions.

the energy and therefore the Lorentz boost available to the charged lepton. Again, though, this makes little difference, since the effects of the boost on the final impact parameter tend to cancel one another. The next chapter will discuss at much greater length other, much more important consequences of requiring visible radiation. 


\section{Chapter 3}

\section{Radiative Production of Heavy Leptons}

This chapter describes the considerations important in understanding radiative prođuction of heavy leptons in $\mathrm{e}^{+} \mathrm{e}^{-}$annihilation. To a large extent, one can separate or factorize the production of the photon from that of the heavy lepton pair. Details. concerning the latter process can be found in chapter 2 and Appendix A. Described here are various techniques for calculating the production rate of two charged leptons and a visible photon, including corrections for extra photons emitted but not detected.

Initially, only electrodynamic processes are considered. The first section gives cross section formulas for producing heavy leptons without radiation. In the second section are given the formulas for bremsstrahlung production of a single photon and for both approximate and exact calculations of the radiative correction to lowest order lepton production. The third section gives a prescription for applying the approximate technique to calculating the radiative correction for the bremsstrahlung process itself. The fourth section describes briefly the effects of weak forces, that is, production of the charged leptons through a virtual $\mathrm{Z}$ boson. Finally, the last section summarizes the cross section values, including corrections, that are used to normalize the estimated predictions from Monte Carlo simulations.

\subsection{Non-radiative Lepton Production}

The lowest order differential cross section for producing two charged leptons of 
mass $\mathrm{M}_{\mathrm{L}}$ from $\mathrm{e}^{+} \mathrm{e}^{-}$annihilation through a virtual photon(see fig. 3.1) is given by:*

$$
\frac{d \sigma\left(\mathrm{e}^{+} \mathrm{e}^{-} \rightarrow \mathrm{L}^{+} \mathrm{L}^{-}\right)}{d \Omega_{\mathrm{L}^{-}}}=\frac{1}{4} \frac{\alpha^{2}}{\mathrm{~s}} \beta\left[1+\cos ^{2} \theta+\left(1-\beta^{2}\right) \sin ^{2} \theta\right]
$$

where $d \Omega_{\mathrm{L}^{-}} \equiv d(\cos \theta) d \phi$, with $\theta$ and $\phi$ the polar and azimuthal angles of the $\mathrm{L}^{-}$, and $\beta$ is the final speed of the charged leptons. $\alpha$ is the fine structure constant $(\approx 1 / 137)$, and $\mathrm{s}$ is the square of the center-of-mass energy. Integrating this expression over the solid angle of $\mathrm{L}^{-}$, one gets the total cross section:

$$
\cdots \quad \sigma_{0}(\mathrm{~s}) \equiv \sigma\left(\mathrm{e}^{+} \mathrm{e}^{-} \rightarrow \mathrm{L}^{+} \mathrm{L}^{-}\right)=\frac{4 \pi}{3} \frac{\alpha^{2}}{\mathrm{~s}}\left[\frac{\beta\left(3-\beta^{2}\right)}{2}\right] \quad ; \quad \beta \equiv \sqrt{1-\left(\frac{\mathrm{M}_{\mathrm{L}}}{\mathrm{E}_{\mathrm{B}}}\right)^{2}}
$$

where $M_{L}$ is the charged lepton mass, and $E_{B}$ is the beam energy. In the limit of small lepton mass, the total cross section approaches:

$$
\sigma_{0}(\mathrm{~s}) \longrightarrow \frac{4 \pi}{3} \frac{\alpha^{2}}{\mathrm{~s}}
$$

This last formula is the familiar "point cross section" for charged fermion-antifermion production, and to a very good approximation, it describes lowest order production of muon and tau pairs at PEP energy. The term in square brackets in eqn. 3.2 is called the threshold factor. For small lepton masses, it is nearly unity, but for very large lepton masses, it approaches zero as the available phase space diminishes.

\subsection{Single Photon Lepton Production}

\subsubsection{Bremsstrahlung}

If the process $e^{+} e^{-} \rightarrow L^{+} L^{-} \gamma$ is now considered, the cross section formulas become significantly more complicated. All four Feynman diagrams in fig. 3.2 and

* Note that terms proportional to the electron mass squared are neglected, where appropriate, in this and other formulas given here. 


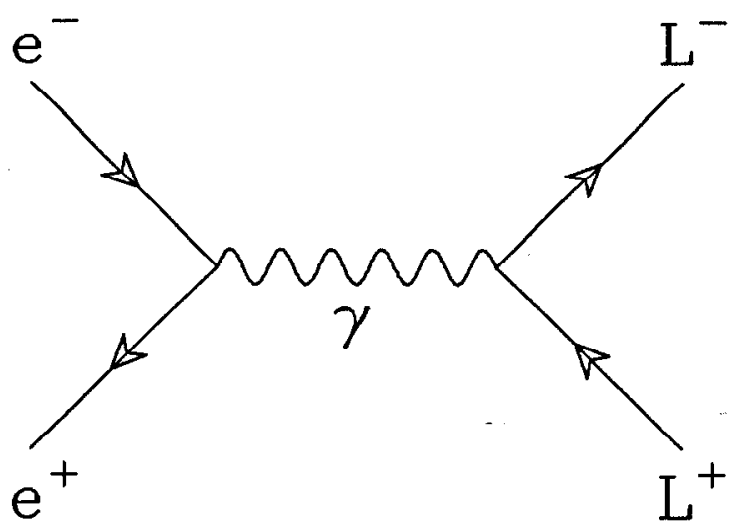

Figure 3.1. Feynman diagram for lowest order production of charged lepton pair through a virtual photon.

their.interferences must be treated. Reference 43 provides the formula:

$$
d \sigma\left(\mathrm{e}^{+} \mathrm{e}^{-} \rightarrow \mathrm{L}^{+} \mathrm{L}^{-} \gamma\right)=\frac{\alpha^{3}}{4 \pi^{2} \mathrm{~s}}\left[\mathrm{~A}_{\text {ini }}+\mathrm{A}_{\text {fin }}+\mathrm{A}_{\text {int }}\right] d \tau
$$

where $d \tau$ is the Lorentz invariant differential phase space volume:

$$
d \tau \equiv \frac{d^{3} \mathrm{q}_{-}}{\mathrm{E}_{-}} \frac{d^{3} \mathrm{q}_{+}}{\mathrm{E}_{+}} \frac{d^{3} \mathrm{k}}{\mathrm{E}_{\gamma}} \delta^{4}\left(\mathrm{p}_{-}+\mathrm{p}_{+}-\mathrm{q}_{-}-\mathrm{q}_{+}-\mathrm{k}\right)
$$

and the initial state, final state and interference contributions are

$$
\begin{aligned}
A_{\text {ins }} \equiv \frac{1}{\hat{s}_{1} x_{1} x_{2}}\left\{\left[\hat{t}^{2}+\hat{u}^{2}+M_{L}^{2} \frac{(\hat{t}+\hat{u})^{2}}{\hat{s}_{1}}\right]\left(1-\frac{m_{e}^{2} x_{1}}{\hat{s}_{1} x_{2}}\right)\right. \\
\left.+\left[\hat{t}_{1}^{2}+\hat{u}_{1}^{2}+M_{L}^{2} \frac{\left(\hat{t}_{1}+\hat{u}_{1}\right)^{2}}{\hat{s}_{1}}\right]\left(1-\frac{m_{\mathrm{e}}^{2} x_{2}}{\hat{s}_{1} x_{1}}\right)\right\} \\
A_{\text {fin }} \equiv \frac{1}{\hat{s}_{1} y_{2}}\left\{\left[\hat{t}^{2}+\hat{u}_{1}^{2}+M_{L}^{2} \hat{s}\right]\left[1-\frac{M_{L}^{2}}{\hat{s}}\left(1+\frac{y_{1}}{y_{2}}\right)\right]\right. \\
+\left[\hat{u}^{2}+\hat{t}_{1}^{2}+M_{L}^{2} \hat{s}\right]\left[1-\frac{M_{L}^{2}}{\hat{s}}\left(1+\frac{y_{2}}{y_{1}}\right)\right] \\
\left.+\frac{M_{L}^{2}}{\hat{s}}\left(x_{1}^{2}+x_{2}^{2}\right)-4 M_{L}^{2}\left(\hat{s}-\hat{s}_{1}\right)\right\}
\end{aligned}
$$




$$
\begin{aligned}
& A_{i n t} \equiv \frac{1}{\sin \theta \hat{s}_{1} x_{1} x_{2} y_{1} y_{2}}\left\{\left(\hat{t} x_{2} y_{2}+\hat{t}_{1} x_{1} y_{1}-\hat{u} x_{2} y_{1}-\hat{u}_{1} x_{1} y_{2}\right)\right. \\
& \times\left[\hat{\mathrm{t}}^{2}+\hat{\mathrm{t}}_{1}^{2}+\hat{\mathrm{u}}^{2}+\hat{\mathrm{u}}_{1}^{2}+\mathrm{M}_{\mathrm{L}}^{2}\left(\hat{\mathrm{s}}+\hat{\mathrm{s}}_{1}\right)\right] \\
& \left.+\mathrm{M}_{\mathrm{L}}^{2} \mathrm{x}_{1} \mathrm{x}_{2}\left[\left(\hat{\mathrm{s}}-\hat{\mathrm{s}}_{1}\right)\left(\hat{\mathrm{t}}+\hat{\mathrm{t}}_{1}-\hat{\mathrm{u}}-\hat{\mathrm{u}}_{1}\right)-\left(\mathrm{x}_{1}-\mathrm{x}_{2}\right)\left(\mathrm{y}_{1}-\mathrm{y}_{2}\right)\right]\right\}
\end{aligned}
$$

where $\mathrm{p}_{-}, \mathrm{p}_{+}, \mathrm{q}_{-}, \mathrm{q}_{+}$, and $\mathrm{k}$ are the 4-momenta of the $\mathrm{e}^{-}, \mathrm{e}^{+}, \mathrm{L}^{-}, \mathrm{L}^{+}$, and $\gamma$, respectively, and $\mathrm{E}_{-}, \mathrm{E}_{+}$, and $\mathrm{E}_{\gamma}$ are the final particle energies. The Lorentz invariant quantities are defined by:*

$$
\begin{gathered}
\hat{\mathrm{s}} \equiv \mathrm{p}_{-} \cdot \mathrm{p}_{+} \quad \hat{\mathrm{t}} \equiv \mathrm{p}_{-} \cdot \mathrm{q}_{-} \quad \hat{\mathrm{u}} \equiv \mathrm{p}_{-} \cdot \mathrm{q}_{+} \\
\hat{\mathrm{s}}_{1} \equiv \mathrm{q}_{-} \cdot \mathrm{q}_{+}+\mathrm{M}_{\mathrm{L}}^{2} \quad \hat{\mathrm{t}}_{1} \equiv \mathrm{p}_{+} \cdot \mathrm{q}_{+} \quad \hat{\mathrm{u}}_{1} \equiv \mathrm{p}_{+} \cdot \mathrm{q}_{-} . \\
\mathrm{x}_{1} \equiv \mathrm{p}_{-} \cdot \mathrm{k} \quad \mathrm{y}_{1} \equiv \mathrm{q}_{-} \cdot \mathrm{k} \\
\mathrm{x}_{2} \equiv \mathrm{p}_{+} \cdot \mathrm{k} \quad \mathrm{y}_{2} \equiv \mathrm{q}_{+} \cdot \mathrm{k}
\end{gathered}
$$

From the structure of eqn. 3.5 and eqn. 3.6 , it is clcar that initial state radiation is most important when $x_{1}$ or $x_{2}$ is small, i.e., when the emitted photon is nearly parallel to the incoming electron or positron. Similarly, final state radiation is most important when $\mathrm{y}_{1}$ or $\mathrm{y}_{2}$ is small, indicating radiation nearly parallel to one of the produced leptons. The relative strengths of the total initial and final state contributions are determined by the mass of the produced leptons. Final state radiation is less important for heavier lepton masses. Classically, this can be understood from the fact that a heavy charged particle suffers less radiation-inducing acceleration than a light particle under the influcnce of the same electromagnetic forces.

Equation 3.4 can be integrated analytically over all solid angle and from some low photon energy, $\mathrm{E}_{\boldsymbol{\gamma}_{\mathrm{min}}}$, to the maximum kinematically allowed photon energy given by:

$$
\mathrm{E}_{\gamma_{\mathrm{max}}}=\mathrm{E}_{\mathrm{B}}\left[1-\left(\frac{\mathrm{M}_{\mathrm{L}}}{\mathrm{E}_{\mathrm{B}}}\right)^{2}\right]
$$

* Reference 43 is missing the exponent on $M_{L}$ in the definition of $\hat{s}_{1}$. 


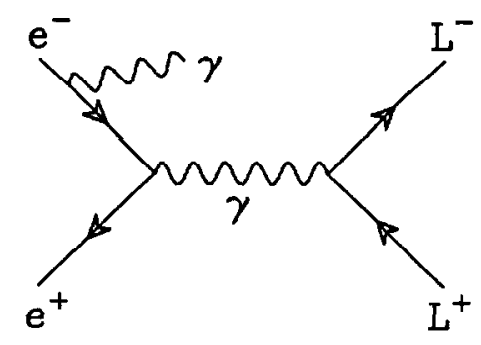

(a)

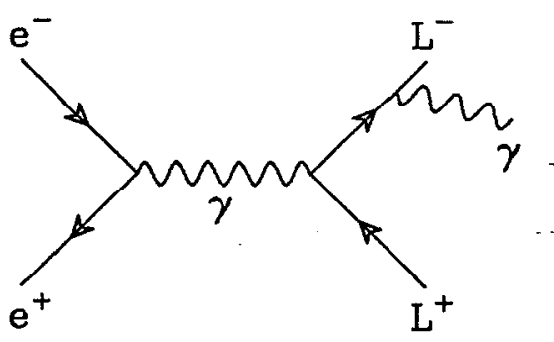

(c)

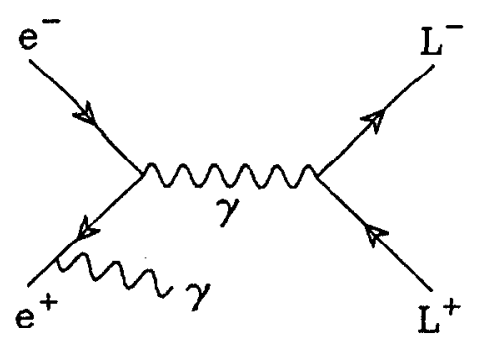

(b)

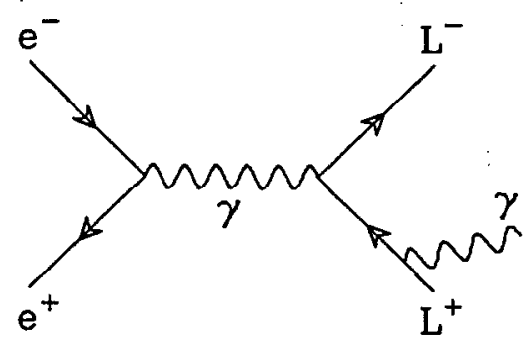

(d)

Figure 3.2. Feynman diagram for lowest order production of charged lepton pair and single photon through a virtual photon

Defining $\kappa_{0} \equiv \mathrm{E}_{\gamma_{\min }} / \mathrm{E}_{\mathrm{B}}, \kappa \equiv \mathrm{E}_{\gamma} / \mathrm{E}_{\mathrm{B}}$, and $\kappa_{\max } \equiv \mathrm{E}_{\gamma_{\max }} / \mathrm{E}_{\mathrm{B}}$, one gets ${ }^{43}$

$$
\begin{aligned}
\sigma_{\text {ini }}\left(\kappa_{0} \leq \kappa \leq \kappa_{\max }\right)=\sigma_{0} \beta_{\text {ini }} & \left\{\ln \frac{1}{\kappa_{0}}-\frac{4}{3}+\ln \frac{4 \beta^{2}}{\mu^{2}}+\frac{1}{\beta\left(3-\beta^{2}\right)} \ln \frac{\mu^{2}}{(1+\beta)^{2}}\right\} \\
\sigma_{\text {fin }}\left(\kappa_{0} \leq \kappa \leq \kappa_{\max }\right)=\sigma_{0}\{ & \beta_{\text {fin }} \ln \frac{1}{\kappa_{0}} \\
+ & \frac{\alpha}{\pi}\left[\frac { 2 ( 1 + \beta ) ^ { 2 } } { \beta } \left[\operatorname{Li}_{2}\left(\frac{1-\beta}{1+\beta}\right)-\operatorname{Li}_{2}\left(\frac{1-\beta}{2}\right)\right.\right. \\
& \left.-\frac{\pi^{2}}{12}-\frac{1}{2} \ln \left(\frac{1+\beta}{2}\right) \ln \left(\frac{(1-\beta)^{2}}{2(1+\beta)}\right)\right] \\
& +\beta\left(3-\beta^{2}\right) \ln \left(\frac{1-\beta^{2}}{4 \beta^{2}}\right)+\frac{39}{8} \beta-\frac{17}{8} \beta^{3} \\
& \left.\left.+\frac{1}{16}\left(9-2 \beta^{2}+\beta^{4}\right) \ln \left(\frac{1+\beta}{1-\beta}\right)\right]\right\}
\end{aligned}
$$


where

$$
\begin{aligned}
\mu \equiv \frac{\mathrm{M}_{\mathrm{L}}}{\mathrm{E}_{\mathrm{B}}} & \beta_{\mathrm{ini}} \equiv \frac{2 \alpha}{\pi}\left[\ln \frac{\mathrm{s}}{\mathrm{m}_{\mathrm{e}}^{2}}-1\right] \\
\mathrm{Y} \equiv \ln \left(\frac{(1+\beta)^{2}}{\mu^{2}}\right) & \beta_{\mathrm{fin}} \equiv \frac{2 \alpha}{\pi}\left[\frac{1+\beta^{2}}{2 \beta} \mathrm{Y}-1\right]
\end{aligned}
$$

and

$$
\operatorname{Li}_{2}(x) \equiv \int_{1-x}^{1} \frac{\ln (1-z)}{z} d z
$$

Taking the limit $\beta \rightarrow 1\left(M_{L} / E_{B} \rightarrow 0\right)$, these expressions become:

$$
\begin{aligned}
& \sigma_{\mathrm{ini}} \rightarrow \sigma_{0} \beta_{\mathrm{ini}}\left\{\ln \left(\frac{1}{\kappa_{0}}\right)+\frac{1}{2} \ln \left(\frac{4}{\mu^{2}}\right)-\frac{4}{3}\right\} \\
& \sigma_{\mathrm{fin}} \rightarrow \sigma_{0}\left\{\beta_{\mathrm{fin}}\left[\ln \left(\frac{1}{\kappa_{0}}\right)-\frac{3}{4}\right]+\frac{\alpha}{\pi}\left[\frac{5}{4}-\frac{\pi^{2}}{3}\right]\right\}
\end{aligned}
$$

with

$$
\beta_{\mathrm{fin}} \rightarrow \frac{2 \alpha}{\pi}\left[\ln \left(\frac{4}{\mu^{2}}\right)-1\right]
$$

For $\kappa_{0}=0.01\left(\mathrm{E}_{\gamma_{\min }} \approx 150 \mathrm{MeV}\right)$, and the lepton mass equal to the muon and tau values, eqn. 3.11 yields

$$
\begin{aligned}
& \sigma_{\text {ini }}(\mu)=(.863) \sigma_{0}(\mu)=89.1 \mathrm{pb} \\
& \sigma_{\text {fin }}(\mu)=(.179) \sigma_{0}(\mu)=18.4 \mathrm{pb} \\
& \sigma_{\text {ini }}(\tau)=(.588) \sigma_{0}(\mu)=60.8 \mathrm{pb} \\
& \sigma_{\text {fin }}(\tau)=(.077) \sigma_{0}(\mu)=8.0 \mathrm{pb}
\end{aligned}
$$

From these numbers one can verify that initial state radiation dominates in the total radiative cross section and that final state radiation falls off more rapidly with increasing lepton mass than does initial state.

It is instructive to consider separately the differential cross sections with respect 
to the photon energy for initial and final state radiation: ${ }^{43}$

$$
\begin{aligned}
& \frac{d \sigma_{\mathrm{ini}}}{d \kappa}=\sigma_{0} \frac{2}{\beta\left(3-\beta^{2}\right)} \beta_{\mathrm{ini}} \frac{1+(1-\kappa)^{2}}{4 \kappa(1-\kappa)} v(\kappa)\left[3-v^{2}(\kappa)\right] \\
& \frac{d \sigma_{\mathrm{fin}}}{d \kappa}=\sigma_{0} \frac{2}{\beta\left(3-\beta^{2}\right)} \frac{\alpha}{\pi} \frac{1}{\kappa}\left\{\left[1+(1-\kappa)^{2}-\mu^{2} \kappa-\frac{\mu^{4}}{2}\right] \ln \left(\frac{1+v(\kappa)}{1-v(\kappa)}\right)\right. \\
&\left.-v(\kappa)\left[\kappa^{2}+\left(2+\mu^{2}\right)(1-\kappa)\right]\right\}
\end{aligned}
$$

where

$$
v(\kappa) \equiv\left(1-\frac{\mu^{2}}{1-\kappa}\right)^{\frac{1}{2}}
$$

These quantities and their sum are plotted in fig. 3.3 for $M_{L}=m_{\mu}, M_{L}=m_{\tau}, M_{L}=$ $8 \mathrm{GeV}$, and $\mathrm{M}_{\mathrm{L}}=12 \mathrm{GeV}$. Both differential cross sections fall off like $1 / \kappa$ at low photon energies, but at very high energies the initial state contribution rises again sharply for small lepton mass. This high energy peak arises mathematically from the $(1-\kappa)$ factor in the denominator of the initial state contribution. Physically, it corresponds to the emission of a very energetic photon, such that the electron and positron collide at a much reduced center of mass energy $\left(=\sqrt{\mathrm{s}^{\prime}}\right)$ where the lowest order cross section for producing a lepton pair becomes quite large. This can be seen from eqn. 3.2 where (ignoring the threshold factor)

$$
\sigma_{0}\left[\mathrm{~s}^{\prime}\right]=\sigma_{0}[\mathrm{~s}(1-\kappa)] \propto \frac{1}{\mathrm{~s}(1-\kappa)}
$$

The enhancement at large photon energies is not seen for heavy leptons because the lowest allowed value of $\mathrm{s}^{\prime}$ is $4 \mathrm{M}_{\mathrm{L}}^{2}$. Put another way, the allowed photon energies fall below the region where the enhancement can occur. One important consequence of lower photon cutoff energies for higher lepton masses is a rcduccd total radiative cross section. Unfortunately, the reduced cross section severely limits the sensitivity of the close-mass lepton search for the heaviest masses, as will become clear in the final chapter. 

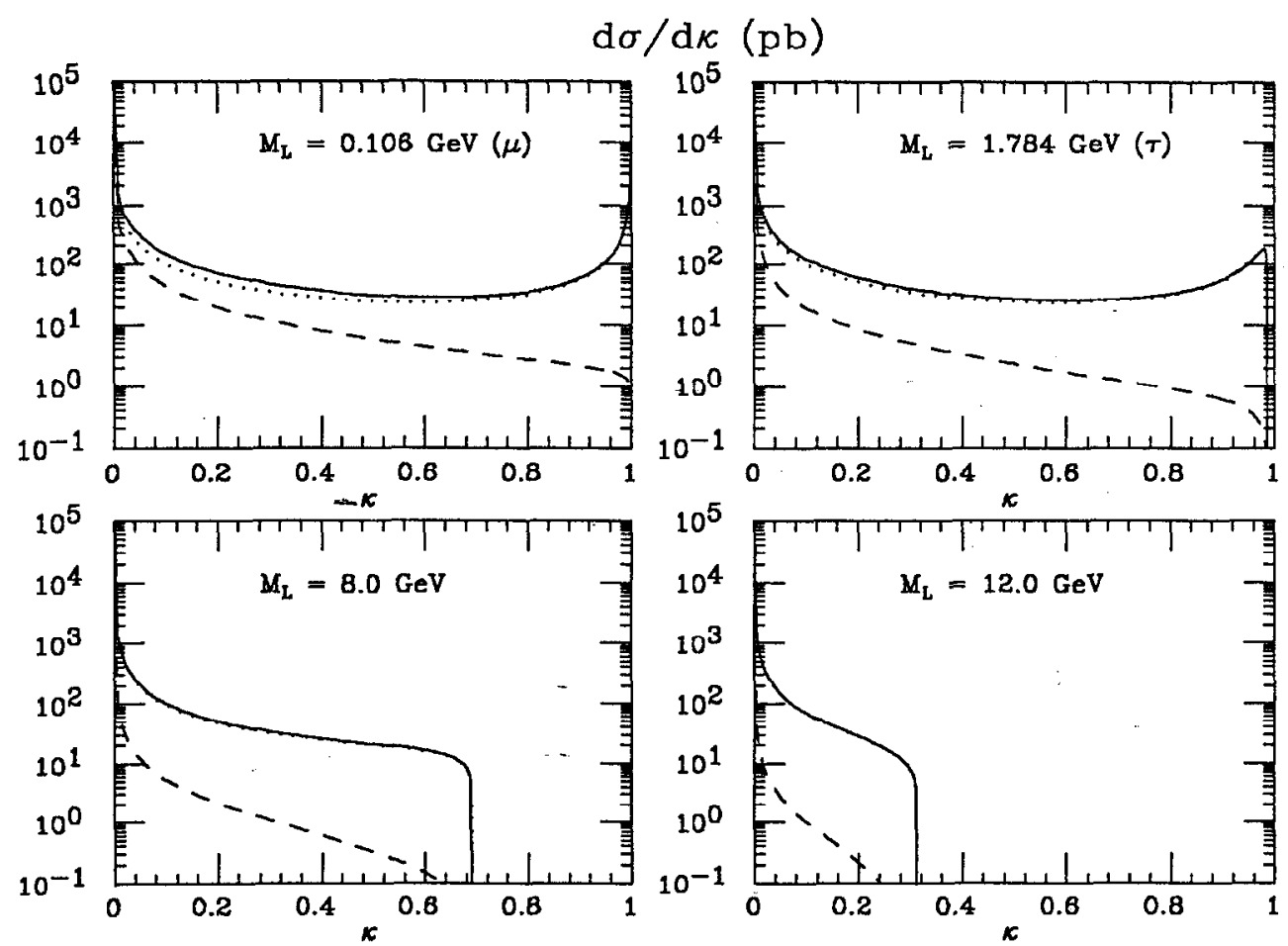

Figure 3.3. Plotted are differential cross sections for initial state(dotted), final state(dashed), and total(solid) radiative production of charged lepton pairs.

\subsubsection{Radiative corrections}

Radiative corrections to a general process account for higher order contributions to the apparent cross section for that process, contributions arising from the emission of one or more extra photons. The presence of these extra photons is unknown because neither they nor their kinematic effect on detected particles is measurable. General, approximate formulas for estimating radiative corrections due to such a single photon emission from the initial electron or positron are presented. Then exact analytic formulas are given for the radiative corrections to the particular process $\mathrm{e}^{+} \mathrm{e}^{-} \rightarrow \mathrm{L}^{+} \mathrm{L}^{-}$. In the next section, the approximate technique will be applied to estimate the radiative correction to the process $\mathrm{e}^{+} \mathrm{e}^{-} \rightarrow \mathrm{L}^{+} \mathrm{L}^{-} \gamma$, where the detected photon appears at large angles with respect to the electron-positron beams.

In calculating radiative corrections to an $\mathrm{e}^{+} \mathrm{e}^{-}$process $\mathrm{e}^{+} \mathrm{e}^{-} \rightarrow \mathrm{X}$, one must con- 
sider the contribution from undetected initial state bremsstrahlung (fig. 3.4 a-b), in which case the formulas given above should in principle apply. Notice, however, that they predict a total cross section that diverges logarithmically as the minimum photon energy approaches zero, a divergence which is unphysical. 'l'he divergence is cancelled only if other contributions of the same order in $\alpha$ are considered. These extra contributions come from the interference between the lowest order process and proccsses involving emission and reabsorption of a virtual photon, as shown in fig. 3.5 a-d. A general, approximate formula is given in ref. 44 for the total radiative correction arising from undetected initial state radiation, both real and virtual:

$$
\begin{aligned}
\sigma_{\mathrm{e}^{+} \mathrm{e}^{-} \rightarrow \mathrm{X} \gamma}(\mathrm{s})= & \sigma_{0}(\mathrm{~s})\left[1+\delta_{\mathrm{app}}\right] \\
& +\beta_{\mathrm{ini}} \int_{\kappa_{0}}^{\kappa_{\mathrm{max}}} \frac{d \kappa}{\kappa}\left(1-\kappa+\frac{1}{2} \kappa^{2}\right) \sigma_{0}(\mathrm{~s}(1-\kappa))
\end{aligned}
$$

where

$$
\delta_{\mathrm{app}} \equiv \beta_{\mathrm{ini}} \ln \left(\kappa_{0}\right)+\frac{2 \alpha}{\pi}\left(\frac{13}{12} \ln \left(\frac{\mathrm{s}}{4 \mathrm{~m}_{\mathrm{e}}^{2}}\right)+\frac{\pi^{2}}{6}-\frac{14}{9}\right)
$$

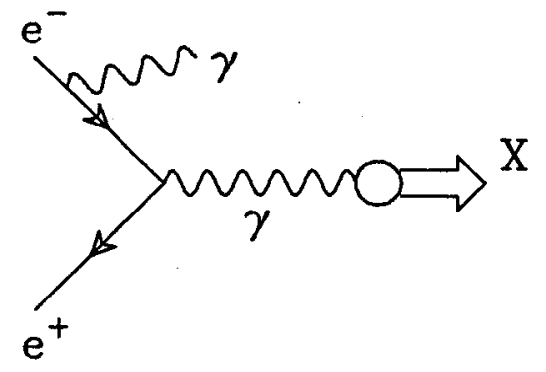

(a)

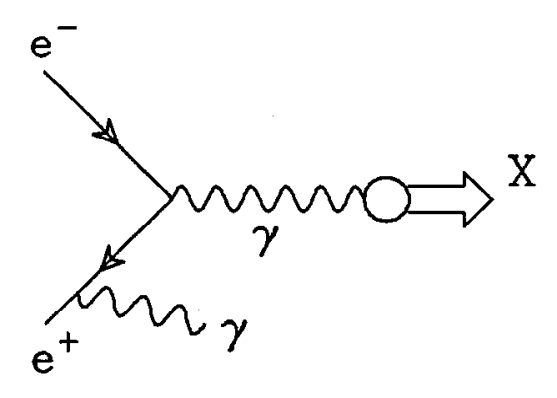

(b)

Figure 3.4. Feynman diagrams for initial state bremsstrahlung production of general system $\mathrm{X}$.

The integral in eqn. 3.13 accounts for the cross section arising from "detectable" photon energies. If one were unable to detect in any way whether an extra photon had been emitted during the production of system $\mathrm{X}$, then eqn. 3.13 , including the 


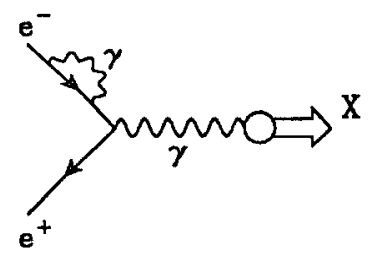

(a)

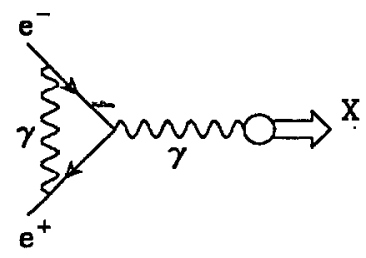

(c)

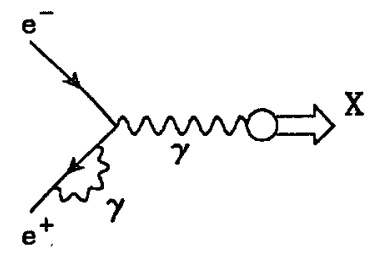

(b)

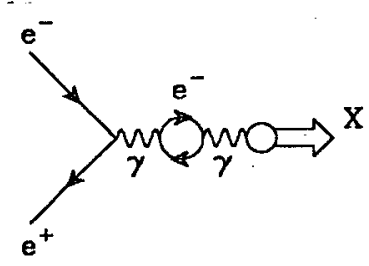

(d)

Figure 3.5. Feynman diagrams for virtual processes that contribute to cross section for producing system X. Note that the vacuum polarization diagram(d) calculated by Bonneau and Martin includes only an electron in the internal fermion loop.

integral, would give the total radiative correction arising from radiation from the initial electron and positron. In practice, however, one can often either detect the extra photon directly or infer its presence from the detected particles $\mathrm{X}$, using conscrvation of momentum and energy. Depending on what cuts are made to select events representing $\mathrm{X}$, the radiative correction may be small or large. The correction can sometimes be estimated analytically for simple processes and simple selection criteria, but in general, one must calculate corrections with a Monte Carlo program.

In the Monte Carlo approach, one generates events corresponding to the total radiatively corrected cross section and then determines what fraction of those events pass final selection criteria. The minimum photon energy is chosen to be a value smaller than both the minimum detectable photon energy and the resolution on the total detected energy. Notice, though, that if $\kappa_{0}$ is small enough, then the term $1+\delta_{\text {app }}$ can become negative. In that extreme, one can either loosen the selection cuts to allow a higher $\kappa_{0}$, which is clearly undesirable, or one can attempt to take into account the emission of multiple photons. The most common approach in this 
situation uses exponentiation of the leading log terms in contributions from all orders in $\alpha$ (see, for example, ref. 45) to account for the extra photons. In this search, however, the missing energy resolution is poor enough(because of missing neutrinos) that $\kappa_{0}=0.01$ is quite satisfactory, ensuring a positive virtual contribution.

When the final state $\mathrm{X}$ is $\mathrm{L}^{+} \mathrm{L}^{-}$, it is possible to calculate analytically the total radiative corrections arising from both initial and final state radiation, including reabsorbed radiation. Figure 3.6 shows additional virtual diagrams included in this exact calculation that are not included in eqn. 3.13 (final state bremsstrahlung, as in fig. $3.2 \mathrm{c}-\mathrm{d}$ are also included). Treated separately are vacuum polarization terms arising from both leptons and quarks in the internal fermion loop (see fig. $3.5 \mathrm{~d}$ ). For emission of a single photon of energy $\mathrm{E}_{\gamma}<\mathrm{E}_{\gamma_{\min }}$, the radiatively corrected cross section is ${ }^{43}$

$$
\begin{aligned}
\sigma_{\mathrm{e}^{+} \mathrm{e}^{-} \rightarrow \mathrm{L}+\mathrm{L}-(\gamma)}\left(\mathrm{E}_{\gamma}<\mathrm{E}_{\gamma_{\min }}\right)=\sigma_{0}[ & {\left[1+\delta_{\mathrm{i}}\left(\mathrm{m}_{\mathrm{e}}^{2}, \kappa_{0}\right)+\delta_{\mathrm{f}}\left(\mathrm{M}_{\mathrm{L}}^{2}, \kappa_{0}\right)+\delta_{\mathrm{VP}}\right.} \\
& \left.+\frac{6}{3-\beta^{2}} \mathrm{~F}_{2}\left(\mu^{2}\right)\right]
\end{aligned}
$$

where

$$
\begin{aligned}
\delta_{\mathrm{i}}\left(\mathrm{m}_{\mathrm{e}}^{2}, \kappa_{0}\right) \equiv & \beta_{\mathrm{ini}} \ln \left(\kappa_{0}\right)+\frac{2 \alpha}{\pi}\left[\frac{3}{4} \ln \left(\frac{4}{\mathrm{~m}^{2}}\right)-1+\frac{\pi^{2}}{6}\right] \\
\delta_{\mathrm{f}}\left(\mathrm{M}_{\mathrm{L}}^{2}, \kappa_{0}\right) \equiv & \beta_{\text {fin }} \ln \left(\frac{2 \kappa_{0}}{\mu}\right)+\frac{2 \alpha}{\pi}\left\{\frac{3+2 \beta^{2}}{4 \beta} \mathrm{Y}-1\right. \\
& \left.-\frac{1+\beta^{2}}{4 \beta}\left[\mathrm{Y}^{2}+4 \operatorname{Li}_{2}\left(\frac{2 \beta}{1+\beta}\right)-\pi^{2}\right]\right\} \\
\mathrm{F}_{2}\left(\mu^{2}\right) \equiv & -\frac{\alpha}{\pi} \frac{\mu^{2}}{4 \beta} \mathrm{Y} .
\end{aligned}
$$

and $\delta_{\mathrm{VP}}$ is the contribution from vacuum polarization of the virtual photon. For each fermion this contribution is ${ }^{46}$

$$
\delta_{\mathrm{VP}}^{\mathrm{f}}\left(\mu_{\mathrm{i}}\right)=\frac{2 \alpha}{\pi} \mathrm{N}_{\mathrm{C}} \mathrm{Q}_{\mathrm{i}}^{2} \mathrm{f}\left(\mu_{\mathrm{i}}^{2}\right) \quad ; \quad \mu_{\mathrm{i}} \equiv \frac{\mathrm{m}_{\mathrm{i}}}{\mathrm{E}_{\mathrm{B}}}
$$


where

$$
f(x)= \begin{cases}-\frac{5}{9}-\frac{x}{3}+\frac{\sqrt{1-x}(2+x)}{6} \ln \left(\frac{1+\sqrt{1-x}}{1-\sqrt{1-x}}\right) ; & x \leq 1 \\ -\frac{5}{9}-\frac{x}{3}+\frac{\sqrt{x-1}(2+x)}{3} \arctan \left(\frac{1}{\sqrt{x-1}}\right) ; & x>1\end{cases}
$$

and

$$
\mathrm{N}_{\mathrm{C}} \equiv \begin{cases}1 & \text { for leptons } \\ 3 & \text { for quarks }\end{cases}
$$

and $Q_{i}$ is the electric charge of the fermion $i$. For $s=(29 \mathrm{GeV})^{2}, x_{i} \ll 1$ for all known leptons and quarks so that

$$
\delta_{\mathrm{VP}}^{\mathrm{f}}\left(\mu_{\mathrm{i}}\right) \approx \frac{2 \alpha}{\pi}\left[\frac{1}{3} \ln \left(\frac{4}{\mu^{2}}\right)-\frac{5}{9}\right]
$$

Note, however, that the exact expressions become relevant in the next section where radiative corrections at significantly reduced c.m. energies must be calculated and where the heavy lepton mass can be comparable to the beam energy. In addition, there are vacuum polarization contributions from hadronic resonances with the same quantum numbers as the virtual photon, such as the $\rho^{0}$. Used in this search is a prescription based on refs. 45,46 , and 47 :

$$
\delta_{\mathrm{VP}}=\sum_{\ell=\mathrm{e}}^{\mu, \tau, \mathrm{L}} \delta_{\mathrm{VP}}^{\mathrm{f}}\left(\mu_{\ell}^{2}\right)+\left(1+\frac{\alpha_{\mathrm{s}}}{\pi}\right) \sum_{\mathrm{q}=\mathbf{u}, \mathrm{d}}^{\mathrm{s}, \mathrm{c}, \mathrm{b}} \delta_{\mathrm{VP}}^{\mathrm{f}}\left(\mu_{\mathrm{q}}^{2}\right)+\sum_{\mathrm{i}} \delta_{\mathrm{VP}}^{\mathrm{h}} \text { (had. res. i) }
$$

where $\alpha_{\mathrm{s}}$ is the strong force coupling constant $(\approx .15)$. For simplicity, the last summation is taken to be a constant $(\approx 1 \%)$, which for these purposes is a good approximation.

Equation 3.9, eqn. 3.10 , and eqn. 3.14 can be summed to give the total radiative corrected cross section ${ }^{43}$

$$
\sigma_{\text {tot }}\left(\mathrm{e}^{+} \mathrm{e}^{-} \rightarrow \mathrm{L}^{+} \mathrm{L}^{-}(\gamma)\right)=\sigma_{0}\left(\mathrm{e}^{+} \mathrm{e}^{-} \rightarrow \mathrm{L}^{+} \mathrm{L}^{-}\right)\left[1+\delta_{\text {ini }}+\delta_{\text {fin }}+\delta_{\mathrm{VP}}\right]
$$

where

$$
\begin{aligned}
\delta_{\mathrm{ini}}= & \beta_{\mathrm{ini}}\left[-\frac{4}{3}+\ln \left(\frac{4 \beta^{2}}{\mu^{2}}\right)+\frac{1}{\beta\left(3-\beta^{2}\right)} \ln \left(\frac{\mu^{2}}{(1+\beta)^{2}}\right)\right] \\
& +\frac{\alpha}{\pi}\left[\frac{3}{2} \ln \left(\frac{4}{\mathrm{~m}^{2}}\right)-2+\frac{\pi^{2}}{3}\right]
\end{aligned}
$$




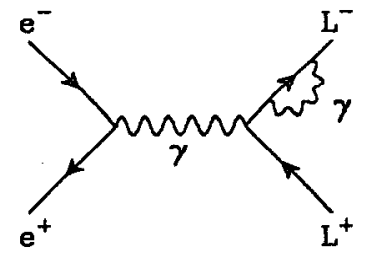

(a)

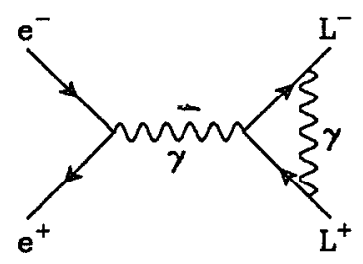

(c)

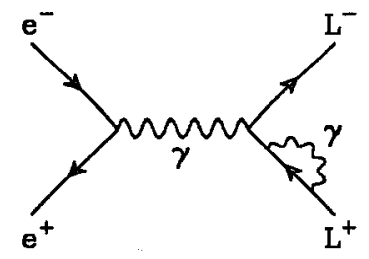

(b)

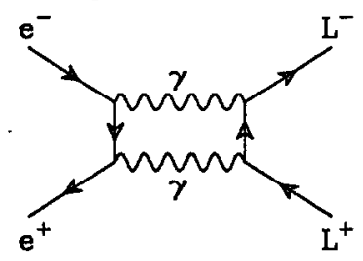

(d)

Figure 3.6. Feynman diagrams for additional virtual processes included in the exact calculation of radiative corrections to $\mathrm{e}^{+} \mathrm{e}^{-} \rightarrow$ $\mathrm{L}^{+} \mathrm{L}^{-}$.

$$
\begin{aligned}
\delta_{\mathrm{fin}}=\frac{\alpha}{\pi}\{ & \frac{2\left(\beta^{2}+1\right)}{\beta}\left[-\ln \left(\frac{1+\beta}{\mu}\right) \ln \left(\frac{2}{1+\beta}\right)-\frac{1}{2} \ln ^{2}\left(\frac{1+\beta}{2}\right)\right. \\
& \left.-\operatorname{Li}_{2}\left(\frac{2 \beta}{1+\beta}\right)+\frac{\pi^{2}}{6}-\operatorname{Li}_{2}\left(\frac{1-\beta}{2}\right)+\operatorname{Li}_{2}\left(\frac{1-\beta}{1+\beta}\right)\right] \\
& -2(1+2 \ln (\beta))+\frac{39-17 \beta^{2}}{4\left(3-\beta^{2}\right)}-\frac{3 \mu^{2}}{2 \beta\left(3-\beta^{2}\right)} \ln \left(\frac{(1+\beta)^{2}}{\mu^{2}}\right) \\
& +\frac{-7 \beta^{4}+24 \beta^{3}+10 \beta^{2}-72 \beta+45}{8 \beta\left(3-\beta^{2}\right)} \ln \left(\frac{4}{\mu^{2}}\right) \\
& \left.+\frac{-7 \beta^{4}+10 \beta^{2}+45}{4 \beta\left(3-\beta^{2}\right)} \ln \left(\frac{1+\beta}{2}\right)\right\}
\end{aligned}
$$

These quantities are plotted in fig. 3.7 vs the charged lepton mass. Although final state bremsstrahlung drops off with increasing lepton mass, the total final state correction, including virtual terms, grows at very high masses because there is a small but non-zero cross section for producing the lepton pair in an s-wave state through emission of a final state photon, a cross section that is not suppressed by the $\beta$ factor present in other contributions. 


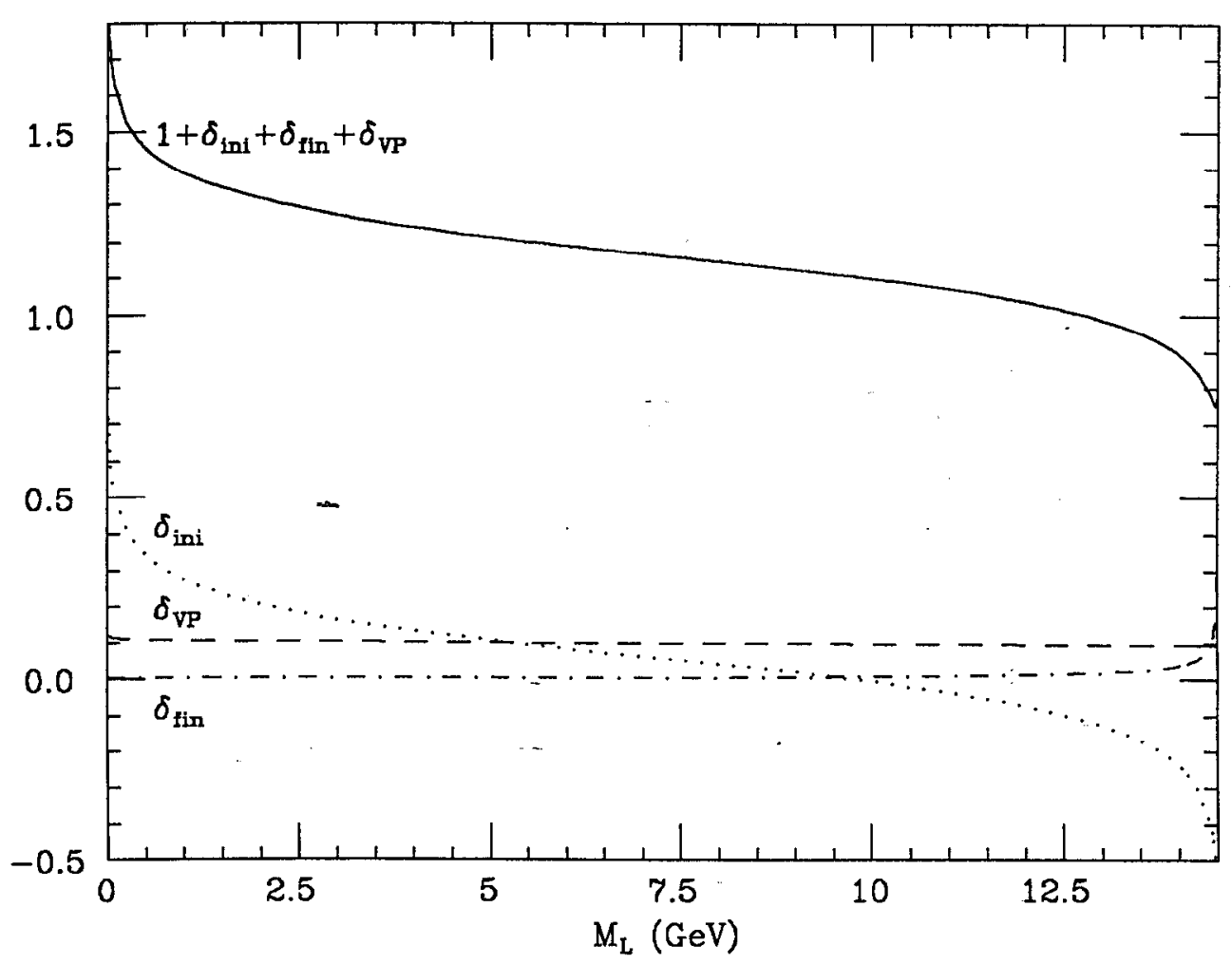

Figure 3.7. Plotted are the initial state(dotted), final state(dotdashed), vacuum polarization(dashed), and total(solid) radiative corrections to $\mathrm{e}^{+} \mathrm{e}^{-} \rightarrow \mathrm{L}^{+} \mathrm{L}^{-}$vs the charged lepton mass.

\subsection{Double Photon Lepton Production}

Equation 3.4 gives the lowest order differential cross section formula for producing charged leptons and a single detected photon. This formula can be used in a Monte Carlo program to calculate the total cross section for producing events that satisfy particular selection criteria. But clearly there can be radiative corrections to the bremsstrahlung process itself, that is, additional, undetected photons can be emitted. Since no exact analytic formulas are currently available which include all relevant diagrams, it is necessary to estimate the corrections with approximate techniques.

The approach taken here is that of Bonneau and Martin ${ }^{44}$ described in the previous section. Only the initial state radiative corrections (see fig. 3.4 and fig. 3.5 ) and full vacuum polarization corrections to the process $\mathrm{e}^{+} \mathrm{e}^{-} \rightarrow \mathrm{X}$ (with $\mathrm{X}=\mathrm{L}^{+} \mathrm{L}^{-} \gamma$ ) are estimated directly. A Monte Carlo program simulates $\mathrm{e}^{+} \mathrm{e}^{-} \rightarrow \mathrm{L}^{+} \mathrm{L}^{-}(\gamma)(\gamma)$ events 
with the Bonneau and Martin approximation, using an iterative technique. At initialization, a discrete lookup table of the integrated photon energy spectrum is generated numerically from eqns. 3.13 and 3.15 , excluding the final state contributions. The lookup table is used to decide randomly for each event whether or not the first, undetected photon is generated, and if so, what energy it is given. As an approximation, the first photon direction is confined to lie along the electron or positron direction. The justification for this approximation is twofold. First, the expected angular distribution is peaked strongly in those directions for reasons described in section 3.2. Second, the assumptions necessary to justify the Bonneau and Martin factorization of the radiative cross section break down for bremsstrahlung production when the first photon is emitted at large angles with respect to the beams. Generating the first photon at large angles in this approach leads to a systematic overestimate of the cross section for producing the second, detected photon at large angles.

Once the energy and direction of the first photon have been generated, then the initial electron and positron(one of which has been perturbed by the photon emission) collide in a new c.m. reference frame that is Lorentz boosted relative to the laboratory frame. In that frame, a new decision must be made whether or not to generate a second photon. The decision and any photon energy generated is determined randomly according to equations $3.9,3.14$, and 3.15 . Note that the minimum photon energy must be recalculated in this frame to be the minimum value that yields a value $\mathrm{E}_{\gamma_{\min }}$ after boosting back to the laboratory frame. The polar angle of the second photon with respect to to the beam axis in the boosted frame is generated according to the distribution ${ }^{45}$

$$
\frac{d \sigma}{d \cos \theta} \propto \frac{2\left(1+(1-\kappa)^{2}\right.}{1-\lambda^{2} \cos ^{2} \theta}-\frac{\mathrm{m}^{2}(1-\kappa)}{(1-\lambda \cos \theta)^{2}}-\frac{\mathrm{m}^{2}(1-\kappa)}{(1+\lambda \cos \theta)^{2}}-\kappa^{2}
$$

where $\lambda^{2} \equiv 1-\mathrm{m}^{2}\left(=1.0-1.2 \times 10^{-9}\right)$. The azimuth angle is generated uniformly. Finally, the heavy leptons are generated in a new c.m. frame. In the new frame their energies are determined uniquely. Their polar angle is generated according to 
eqn. 3.1 with respect to to the beam axis in that frame, and again the azimuth angle is generated uniformly.

In general, to determine the radiatively corrected cross section with this technique, one generates events according to the total cross section and finds the fraction that pass final selection criteria, where full simulation of lepton decay and daughter particle interactions with the detector is performed. For this analysis, however, the primary heavy lepton Monte Carlo program(described in Appendix A) simulates only initial state single photon emission. One can obtain a good estimate of the radiative corrections, howcver, by imposing relatively simple selection criteria on the detectcd photon and the undecayed charged leptons, criteria designed to match those used in the actual analysis described in the final chapter. By comparing the number of passing events with and without simulation of the extra, undetected photon, one can indirectly estimate the radiative corrections. The error on this estimate can itself be estimated by varying the exact criteria used to define a passing event and measuring the sensitivity of the radiative correction to those criteria. The results of this study are presented in the final section of this chapter.

\subsection{Weak Effects}

Electrons and positrons can annihilate with each other not only through a virtual photon, but also through a virtual $\mathrm{Z}$ boson, the mediator of the weak force. In lowest order lepton pair production, one must in principle consider not only the diagram in fig. 3.1, but also that in fig. 3.8 and the interference between the two. Exact formulas are given in appendix A, including contributions arising from correlated spin effects in the decays of the leptons. For now, it is useful to consider simplified formulas in which spin effects are ignored. The generalization of eqn. 3.1 that takes into account 
lowest order weak effects is

$$
\begin{aligned}
& \frac{d \sigma\left(\mathrm{e}^{+} \mathrm{e}^{-} \rightarrow \mathrm{L}^{+} \mathrm{L}^{-}\right)}{d \Omega_{\mathrm{L}^{-}}}=\frac{1}{4} \frac{\alpha^{2}}{\mathrm{~s}} \beta\left[\left(1+\cos ^{2} \theta+\left(1-\beta^{2}\right) \sin ^{2} \theta\right)\right. \\
& +\frac{1}{8 \sin ^{2} \theta_{\mathrm{W}} \cos ^{2} \theta_{\mathrm{W}}} \frac{\mathrm{s}\left(\mathrm{s}-\mathrm{M}_{\mathrm{Z}}^{2}\right)}{\left(\mathrm{s}-\mathrm{M}_{\mathrm{Z}}^{2}\right)^{2}+\mathrm{M}_{\mathrm{Z}}^{2} \Gamma_{\mathrm{Z}}^{2}} \\
& \times\left\{\left(1-4 \sin ^{2} \theta_{\mathrm{W}}\right)^{2}\left(1+\cos ^{2} \theta+\left(1-\dot{\beta}^{2}\right) \sin ^{2} \theta\right)+2 \beta \cos \theta\right\} \\
& +\frac{-1}{64 \sin ^{4} \theta_{\mathrm{W}} \cos ^{4} \theta_{\mathrm{W}}} \frac{\mathrm{s}^{2}}{\left(\mathrm{~s}-\mathrm{M}_{\mathrm{Z}}^{2}\right)^{2}+\mathrm{M}_{\mathrm{Z}}^{2} \Gamma_{\mathrm{Z}}^{2}} \\
& \times\left\{\left(1-4 \sin ^{2} \theta_{\mathrm{W}}+8 \sin ^{4} \theta_{\mathrm{W}}\right)^{2}\left(1+\beta^{2} \cos ^{2} \theta\right)\right. \\
& -4\left(1-\beta^{2}\right)\left(1-4 \sin ^{2} \theta_{\mathrm{W}}+8 \sin ^{4} \theta_{\mathrm{W}}\right)\left(\sin ^{2} \theta_{\mathrm{W}}-2 \sin ^{4} \theta_{\mathrm{W}}\right) \\
& \left.\left.-2\left(1-4 \sin ^{2} \theta_{\mathrm{W}}\right)^{2} \beta \cos \theta\right\}\right]
\end{aligned}
$$

where $M_{Z}$ and $\Gamma_{Z}$ are the mass and total decay width of the $Z$ boson, and $\theta_{W}$ is the weak mixing angle. Substituting the values $\mathrm{M}_{Z}=93 \mathrm{Gev}, \Gamma_{Z}=2.7 \mathrm{GeV}$, and $\sin ^{2} \theta_{\mathrm{W}}=0.22$, this becomes at PEP energy:

$$
\begin{aligned}
\frac{d \sigma\left(\mathrm{e}^{+} \mathrm{e}^{-} \rightarrow \mathrm{L}^{+} \mathrm{L}^{-}\right)}{d \Omega_{\mathrm{L}^{-}}}=\frac{1}{4} \frac{\alpha^{2}}{\mathrm{~s}} \beta\{ & \left(1+\cos ^{2} \theta+\left(1-\beta^{2}\right) \sin ^{2} \theta\right) \\
& -(.0011)\left(1+\cos ^{2} \theta+\left(1-\beta^{2}\right) \sin ^{2} \theta\right)+(.16) \beta \cos \theta \\
& +(.0016)\left(1+\beta^{2} \cos ^{2} \theta\right)-(.0015)\left(1-\beta^{2}\right) \\
& -.00017 \beta \cos \theta\}
\end{aligned}
$$

Clearly, weak effects at PEP energy are negligible in the total cross section, which depends only on the terms even in $\cos \theta$. There is a measurable forward-backward asymmetry arising from the terms proportional to $\cos \theta$. The Monte Carlo program used to simulate signal and tau pair backgrounds correctly simulates this asymmetry and accounts for a minute change in the photon energy spectrum, arising from the weak corrections to the total lowest order cross section at reduced c.m. energies. 


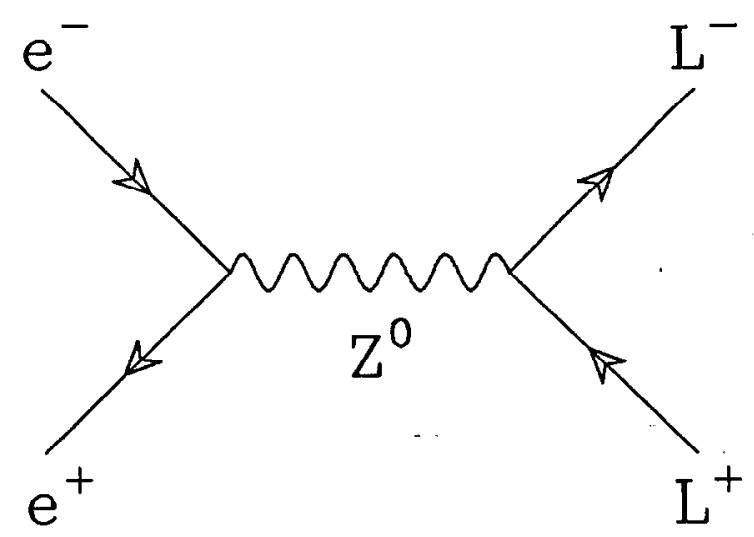

Figure 3.8. Feyñman diagram of charged lepton production through $\mathrm{e}^{+} \mathrm{e}^{-}$annihilation into a virtual $\mathrm{Z}$ boson.

\subsection{Monte Carlo Corrections}

In this section are summarized the corrections that must be applied to results obtained from the Monte Carlo program described in Appendix A for simulating radiative heavy lepton production, including production of tau pairs. The program simulates only initial state radiation and includes no radiative correction to the bremsstrahlung process.

Since selection cuts that require the detected photon be emitted at large angles with respect to the beam tend to enhance the otherwise small proportion of final state radiation events, it is necessary to apply a correction to each Monte Carlo generated event weight. This correction is calculated according to

$$
\mathrm{W}_{\mathrm{fin} / \mathrm{ini}}=\frac{\mathrm{A}_{\mathrm{ini}}+\mathrm{A}_{\mathrm{fin}}+\mathrm{A}_{\mathrm{int}}}{\mathrm{A}_{\mathrm{ini}}}
$$

where the $\mathrm{A}$ terms are defined in section 3.2. The distributions of $\mathrm{W}_{\mathrm{fin}}$ for the radiative tau pair background and for three choices of $\left(\mathrm{M}_{\mathrm{L}}, \mathrm{M}_{\nu_{\mathrm{L}}}\right)$ are shown in fig. 3.9 for events passing the final selection criteria described in chapter 5. From these distributions, one can see that final state radiation gives a small but significant contribution to the tau background and to the signal for lighter leptons.

Radiative corrections to $\mathrm{e}^{+} \mathrm{e}^{-} \rightarrow \mathrm{L}^{+} \mathrm{L}^{-} \gamma$ are estimated with the relatively crude Monte Carlo program described in section 3.3, where only initial state and vacuum 


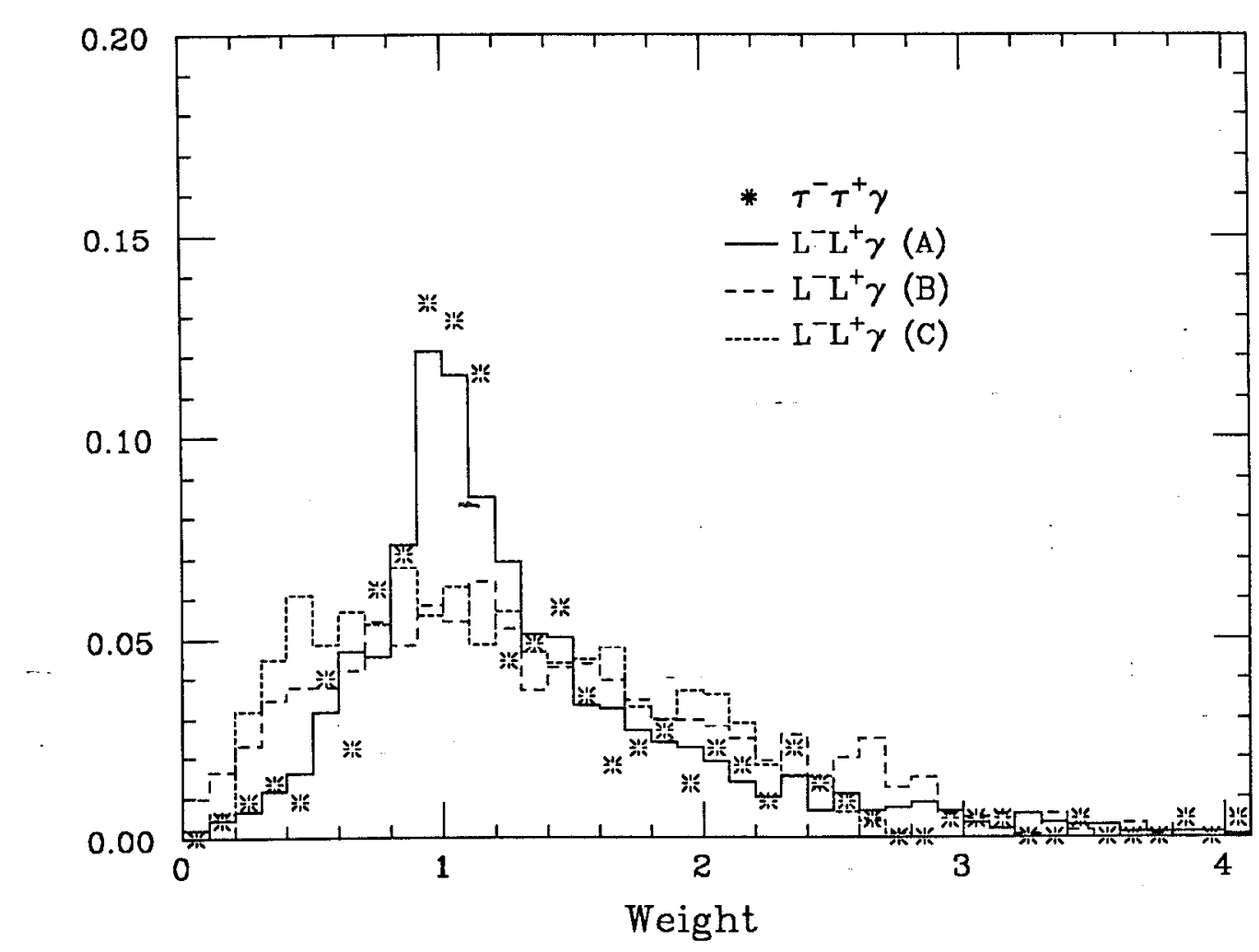

Figure 3.9. Distributions in final state radiation event weight correction for Monte Carlo events passing selection criteria described in final chapter. Distributions are normalized to unity. The plotted points are for radiative tau background, and the histograms $\mathrm{A}, \mathrm{B}, \mathrm{C}$ are for heavy charged lepton masses of 2,6 , and $10 \mathrm{GeV}$, respectively, with $\delta_{\mathrm{m}}=400 \mathrm{MeV}$ for all three.

polarization corrections are considered. The radiative correction to a process depends on exactly what selection criteria define the process. To estimate the radiative correction without performing full-blown simulation of lepton production and decay, various sets of conditions are imposed on the undecayed leptons, sets designed to match closely those actually imposed on the reconstructed daughter tracks. The corrections for these different sets of conditions are found empirically, as described earlier, and the error on the estimate inferred from the variation in corrections among the different sets.

Table 3.1 shows the radiative corrections for producing $\mathrm{L}^{+} \mathrm{L}^{-} \gamma$ for several lepton masses and for various combinations of the following conditions: 
Table 3.1. Radiative corrections to bremsstrahlung production for various selection criteria, calculated from modified Bonneau and Martin approximation.

\begin{tabular}{|c|c|r|r|r|r|r|r|}
\hline \multicolumn{2}{|c|}{} & \multicolumn{6}{|c|}{ Lepton Mass (GeV) } \\
\hline Set & Criteria & .10566 & 1.784 & \multicolumn{1}{|c|}{4.0} & \multicolumn{1}{c|}{8.0} & 12.0 & 13.0 \\
\hline A & I,II & 1.24 & 1.19 & 1.16 & 1.08 & 0.96 & 0.86 \\
& & \pm .01 & \pm 0.01 & \pm 0.01 & \pm 0.01 & \pm 0.01 & \pm 0.01 \\
\hline B & I,II,III & 1.25 & 1.19 & 1.16 & 1.07 & 0.96 & 0.88 \\
& & 0.01 & \pm 0.01 & \pm 0.01 & \pm 0.01 & \pm 0.01 & \pm 0.01 \\
\hline C & I,II,IV & 1.35 & 1.32 & 1.28 & 1.17 & 1.03 & 0.91 \\
& & \pm 0.01 & \pm 0.01 & \pm 0.01 & \pm 0.02 & \pm 0.02 & \pm 0.02 \\
\hline D & I,II,V & 1.11 & 1.11 & 1.09 & 1.04 & 0.94 & 0.86 \\
& & \pm 0.01 & \pm 0.01 & \pm 0.01 & \pm 0.01 & \pm 0.01 & \pm 0.02 \\
\hline E & I,II,III,IV & 1.32 & 1.30 & 1.24 & 1.14 & 1.02 & 0.91 \\
& & \pm 0.01 & \pm 0.01 & \pm 0.01 & \pm 0.02 & \pm 0.02 & \pm 0.02 \\
\hline F & I,II,III,V & 1.12 & 1.12 & 1.09 & 1.03 & 0.93 & 0.88 \\
& & \pm 0.01 & \pm 0.01 & \pm 0.01 & \pm 0.01 & \pm 0.02 & \pm 0.02 \\
\hline G & I,II,IV,V & 1.18 & 1.20 & 1.17 & 1.14 & 1.03 & 0.89 \\
& & \pm 0.02 & \pm 0.02 & \pm 0.02 & \pm 0.03 & \pm 0.03 & \pm 0.04 \\
\hline H & I,II,III,IV,V & 1.16 & 1.18 & 1.14 & 1.11 & 1.02 & 0.88 \\
& & \pm 0.02 & \pm 0.02 & \pm 0.02 & \pm 0.03 & \pm 0.03 & \pm 0.04 \\
\hline
\end{tabular}

I) $\left|\cos \theta_{\gamma}\right| \leq 0.70$

II) $\mathrm{E}_{\gamma} \geq 850 \mathrm{MeV}$

III) $\operatorname{Max}\left(\cos \theta_{\gamma_{L^{+}}}, \cos \theta_{\gamma_{L^{-}}}\right) \leq 0.70$ where the angles are between the photon and each of the charged lepton directions.

IV) $20^{\circ} \leq \theta_{\mathrm{L}+\mathrm{L}^{-}} \leq 160^{\circ}$ where the angle is between the directions of the charged leptons. 
V) $\operatorname{Max}\left[\left|\cos \theta_{L_{+}}\right|,\left|\cos \theta_{L^{-}}\right|\right] \leq 0.70$ where the angles are between each charged lepton and the beam direction.

Set $\mathrm{H}$, which imposes all five conditions, corresponds most closely to the selection criteria used in the final analysis. Consequently, a simple analytic curve was fit to the radiative corrections found for this set, using the values and errors listed in Table 3.1(along with others not shown). The result of the fit is shown in fig. 3.10.

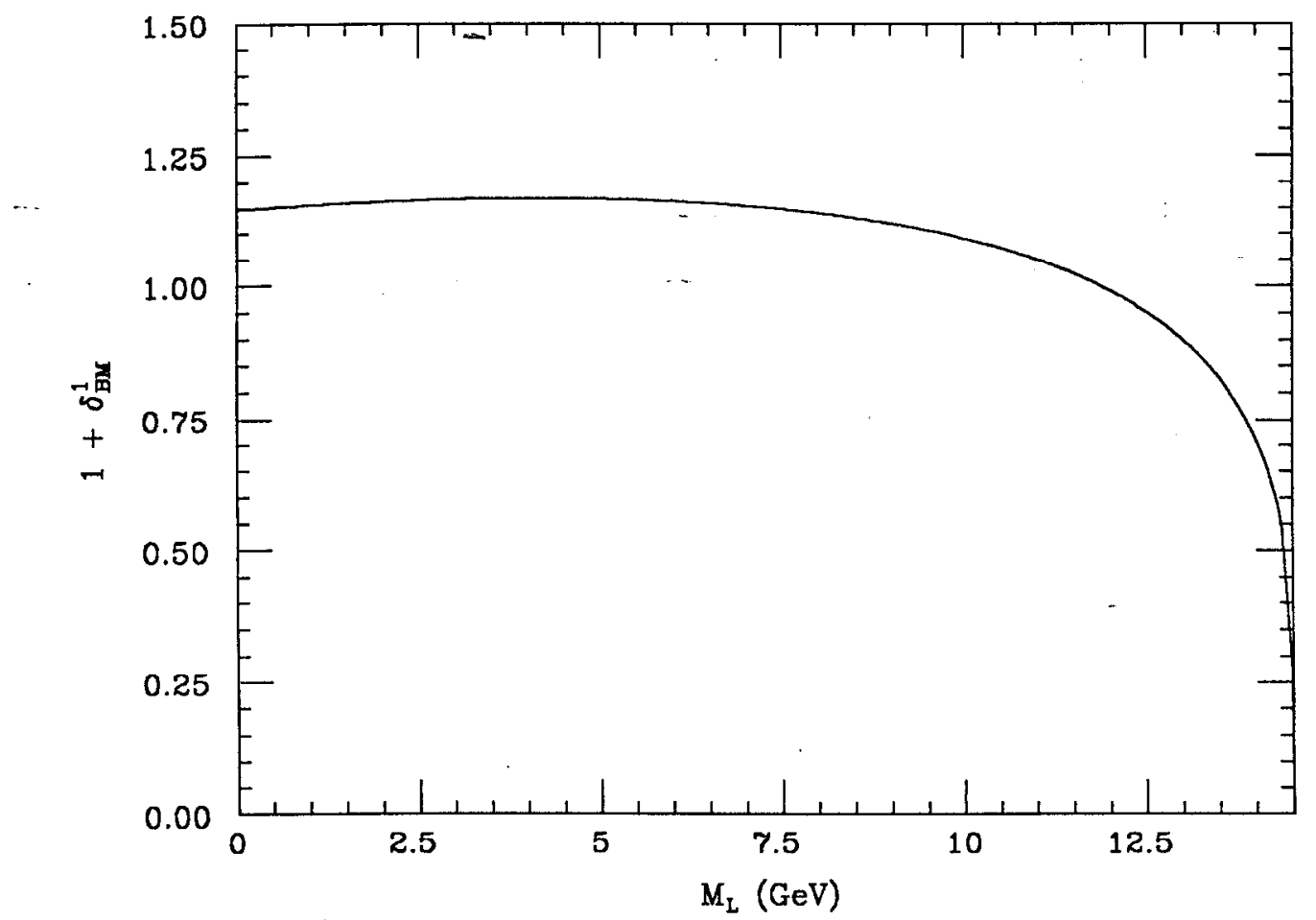

Figure 3.10. Plotted vs charged lepton mass is the estimated radiative correction to bremsstrahlung calculated for the conditions imposed in set $\mathrm{H}$.

There are two potentially significant sources of error in calculating the radiative corrections to $\mathrm{e}^{+} \mathrm{e}^{-} \rightarrow \mathrm{L}^{+} \mathrm{L}^{-} \gamma$ with this approach. The first is that the selection criteria chosen cannot cxactly match those uscd in the final analysis. This crror can be estimated from the range of values found with the various sets of criteria listed in Table 3.1. From the table, an uncertainty of $10 \%$ appears to be a reasonable, conservative estimate of the error. 
The second potential source of error is the omission of the final state radiative correction (note: this is not the same as the correction applied event-by-event to account for detected final state radiation). Omission of this correction is safe, however. This can be argued from fig. 3.7, where the total final state correction for lowest order lepton production (dot dashed curve) is very small. The addition of a detected photon to the lowest order process should not significantly increase this small correction.

In summary, the radiative correction to $\mathrm{e}^{+} \mathrm{e}^{-} \rightarrow \mathrm{L}^{+} \mathrm{I}^{-} \gamma$ and its error is taken to be:

$$
\sigma\left(\mathrm{e}^{+} \mathrm{e}^{-} \rightarrow \mathrm{L}^{+} \mathrm{L}^{-} \gamma(\gamma)\right)=\sigma\left(\mathrm{e}^{+} \mathrm{e}^{-} \rightarrow \mathrm{L}^{+} \mathrm{L}^{-} \gamma\right) \times\left(1+\delta_{\mathrm{BM}}^{1} \pm 0.10\right)
$$

where $\delta_{\mathrm{BM}}^{1}$ is the estimated radiative correction to detected bremsstrahlung, as calculated with the modified Bonneau and Martin approximation, including full vacuum polarization.

In order to increase program efficiency in signal event generation, not all of the lowest order bremsstrahlung process is simulated. Bounds are placed on the photon energy spectrum and the photon's polar angle with respect to the beam direction, bounds that are compatible with the final selection criteria. The constraints on generated events are

$$
\begin{aligned}
\mathrm{E}_{\gamma} & \geq 850 \mathrm{MeV} \\
\left|\cos \left(\theta_{\gamma}\right)\right| & \leq 0.70
\end{aligned}
$$

The lowest order cross section for these cuts is plotted in fig. 3.11, along with the radiatively corrected cross section, calculated from eqn. 3.19 . These radiatively corrected cross section values are the ones used to normalize the samples of generated heavy lepton signal and tau pair background events.*

* One minor point is that for new heavy lepton production, the lepton itself contributes to the vacuum polarization, a contribution not included in the calculation of background tau production. 


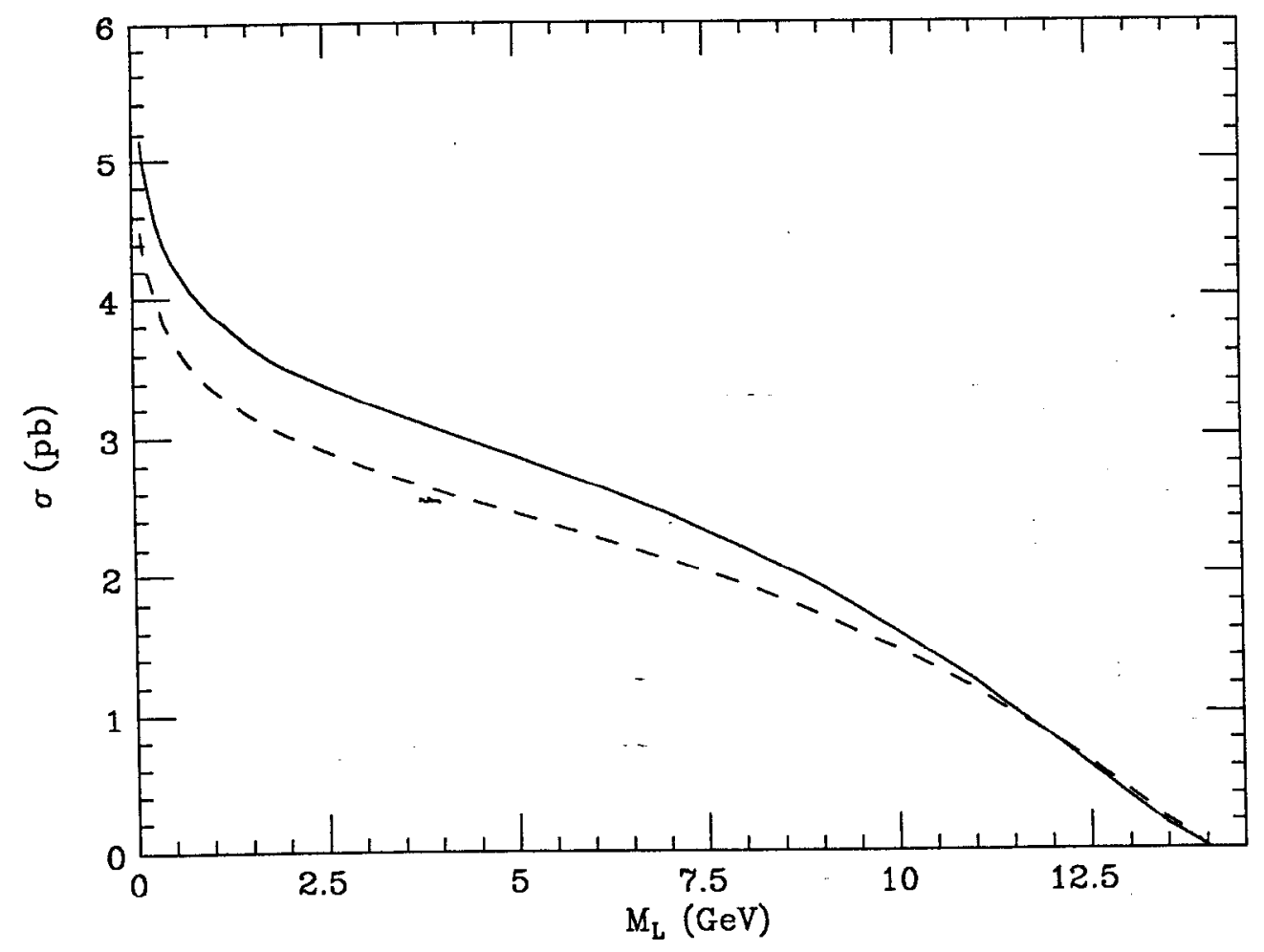

Figure 3.11. Plotted are total bremsstrahlung cross sections for the restricted photon energies and polar angles described in the text. The dashed curve is a calculation of the exact lowest order initial state contribution, and the solid curve is the initial state contribution after radiative correction by eqn. 3.19 . Note that these cross sections do not include the contribution from detected final state bremsstrahlung, for which the normalization correction is calculated event by event. 


\section{Chapter 4}

\section{PEP and the Mark II Detector}

This chapter describes briefly the characteristics of the PEP electron-positron storage ring and in some detail the elements of the Mark II detector important to this work. Where appropriate, the performance of those elements is discussed, especially their usefulness and sensitivity in detecting close-mass lepton events.

\subsection{The PEP Storage Ring}

This analysis is performed on data taken during the period 1981-1984 with the Mark II detector, located in one of the interaction halls of the PEP(Positron Electron Project) storage ring. Shaped like a rounded off hexagon, the PEP ring has a circumference of $2.2 \mathrm{~km}$. During this data-taking period, PEP usually stored three electron and three positron bunches, each providing a current of several $\mathrm{mA}$ with beam energies of $14.5 \mathrm{GeV}$, giving total collision energies of $29 \mathrm{GeV}$ at the six interaction halls.

Under its best running conditions, PEP supplied instantaneous luminosities above $10^{31} \mathrm{~cm}^{-2} \mathrm{~s}^{-1}$ and daily integrated luminosities of about $1 \mathrm{pb}^{-1}$. The total integrated luminosity accumulated by the complete Mark II detector before its shutdown for improvements was just over $200 \mathrm{pb}^{-1}$. Because of degraded drift chamber performance during part of this period, which will be discussed later in this chapter, only the second half of the $\operatorname{data}\left(104 \mathrm{pb}^{-1}\right)$ is used in this analysis. 


\subsection{Overview of Mark II Detector}

During the early years, most of the interaction halls at PEP were occupied by sophisticated high energy particle detectors, each with its own strengths and weaknesses. The Mark II (shown in fig. 4.1) was designed as a general purpose detector, providing versatility in measuring many diverse particle processes. First operated at the lower-energy SPEAR storage ring, the detector has now been moved from PEP to the interaction hall of the very high energy SLAC Linear Collider (SLC), where it should soon detect interactions involving the production and decay of the $\mathrm{Z}^{0}$ gauge boson. Although throughout its history the Mark II has been steadily upgraded, its basic design as a general purpose detector has remained the same.

Given in this section is a brief overview of the Mark II detector. Succeeding sections give a more detailed description of each major part of the detector that is important to this analysis. Further information concerning the hardware, including more detailed mechanical and electronic specifications, can be found elsewhere. ${ }^{48,49}$

Charged particle tracking is provided by two cylindrical drift chambers concentric with the beam line. The inner drift chamber(vertex chamber) gives excellent spatial resolution near the interaction region in order to identify particles originating from decays that occur away from the beam collision region. The large outer chamber provides good momentum resolution in a magnetic field of $2.35 \mathrm{kG}$. Just within the

cylindrical magnetic coil are 48 plastic scintillators in long strips parallel to the beam, which provide time-of-flight(TOF) information for charged particles. Outside of the magnetic coil are eight lead-liquid argon calorimeter modules which primarily measure the energies of electromagnetic showers from electrons and photons. Surrounding the calorimeter are four layers of steel and proportional tubes, allowing high energy muons to be distinguished from charged hadrons. In each forward region is a small angle tagging(SAT) detector that consists of three sets of planar drift chambers, a lead-scintillator sandwich calorimeter and a set of coincidence scintillators. Together, the SAT elements allow tagging of large q-squared two-photon events and provide a 

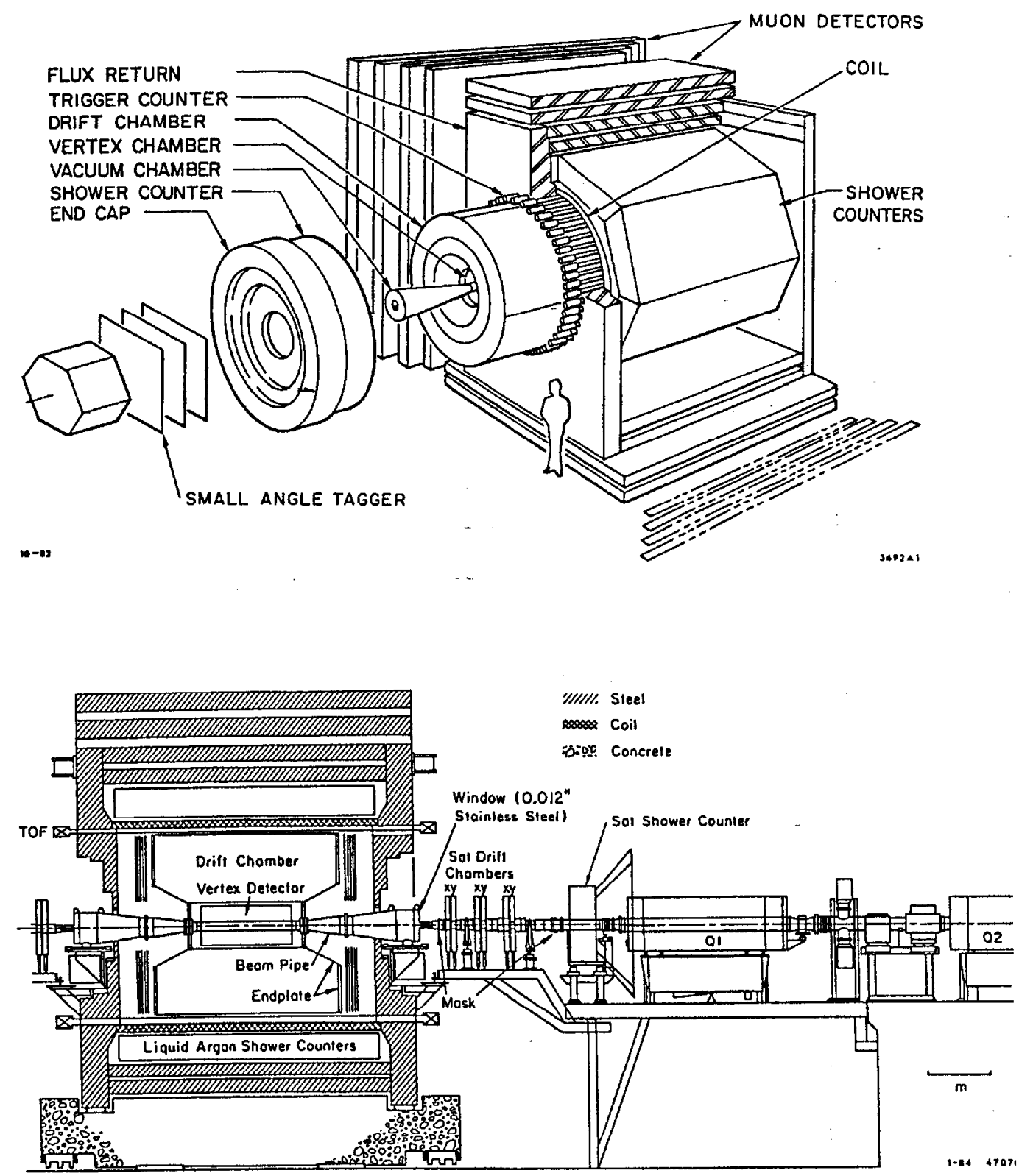

Figure 4.1. The top figure shows an exploded, perspective view of the Mark II detector. Below is a side view, where the electrons enter the detector from the right through the beampipe and the quadrupole magnets marked Q1 and Q2. The positrons enter from the left through an identical system, not shown.

fairly accurate measure of integrated luminosity. In addition, end caps made from sandwiches of lead and proportional tubes provide calorimetry for low angle electrons and photons. Unfortunately, poor energy resolution, shower leakage due to thinness, 
and large holes in acceptance, limit the usefulness of these end caps. They are not used in this analysis.

\subsection{Vertex Chamber}

The inner vertex chamber( $\mathrm{VC})$ is constructed to optimize spatial resolution for identifying charged particles originating from decays. Its sensitive region is divided into 2 concentric bands of axial sense wires, parallel to the beam. The inner band has four layers of sense wires at an average radius of $11.4 \mathrm{~cm}$, with a inter-layer spacing of $8 \mathrm{~mm}$. The outer band has three layers at an average radius of $31.2 \mathrm{~cm}$ with about the same inter-layer spacing. Nearby to the sense wires are parallel field wires, held at various high voltages to produce carefully designed electric fields. The length of the active vertex chamber is $1.26 \mathrm{~m}$.

When a charged particle passes through the chamber it ionizes gas molecules, releasing electrons which drift in the electric field toward the sense wires. These electrons avalanche in the extremely high field surrounding the thin sense wires, inducing an appreciable electrical signal on the wire. The time the signal occurs is recorded by readout electronics, providing information on how near the original charged particle came to the sense wire, given the known drift velocity of electrons in the gas. The electric fields are designed so that a single charged particle should cause a signal on only one sense wire per layer. There remains an ambiguity, however, in that a charged track passing an equal distance to either side of a given sense wire will produce in each case the same signal. This left-right ambiguity can usually be resolved with information from other layers, but it increases processing time during track reconstruction, as it increases the number of possible trajectories to be considered. Occasionally, the ambiguity is resolved incorrectly in the software and leads to degradation of both momentum and spatial resolution. Figure 4.2 shows the sensc and field wire pattern in one sector of the inner band of wires.

All seven VC layers are axial, that is, parallel to the beam, which means they 


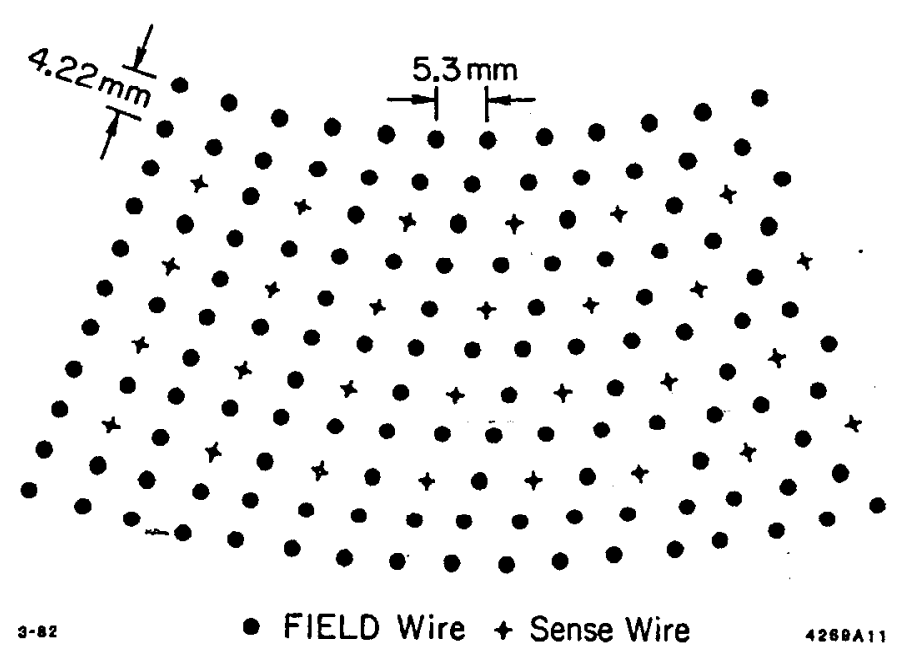

Figure 4.2. The figure shows the pattern of sense and field wires for one sector of the vertex chamber's inner band.

provide information on the charged particle coordinates in the plane $(x-y)$ transverse to the beam, but not on the z-coordinate, parallel to the beam. The innermost layer has 60 sense wires and the outermost has 190.

\subsection{Outer Drift Chamber}

The outer drift chamber has 16 layers of sense wires. Six layers of axial wires alternate with 10 layers of stereo wires, grouped in five pairs with tilt equal to $\pm 3^{\circ}$. The combination of axial and stereo wire hits allows a fairly reliable reconstruction of the z-coordinates of charged particle trajectories. The layers are spaced uniformly in radius from $41 \mathrm{~cm}$ to $145 \mathrm{~cm}$ and have lengths ranging from 1.98 to $2.78 \mathrm{~m}$.

Since both chambers are immersed in a $2.35 \mathrm{kG}$ field, charged particles passing through are bent into trajectories that, to a good approximation, describe helices. The radius of curvature of such a helix is a direct measure of the magnitude of the particle's momentum transvcrsc to the beam $\left(p_{\perp}\right)$. The polar angle information derived from the stereo wires then allows a measure of the total momentum of the particle. By reconstructing the entire helix, one can deduce the direction of the particle as it left the vicinity of the interaction region. Henceforth, unless otherwise 
stated, the momentum of a charged track is understood to refer to this original vector.

It can be shown that under realistic conditions, the resolution on the transverse momentum of a charged particle in a magnetic field, as measured by a drift chamber is well approximated by

$$
\frac{\delta_{p_{\perp}}}{p_{\perp}}=\left[a p^{2}+b\right]^{\frac{1}{2}}
$$

where $a$ and $b$ are parameters depending on the amount of material through which the particle must pass, on the exact configuration of wires, including their intrinsic space resolution, and on the strength of the magnetic field. At high momentum, the first term dominates and arises from the resolution on the sagitta of the sector of the helix contained in the chamber. The second term dominates at lower momenta where multiple scattering, primarily at the beam pipe and boundary between the inner and outer drift chambers, degrades the momentum resolution. The parameters $a$ and $b$ can be measured directly either from cosmic ray muons passing through the interaction point or from beam-produced particles of well-known energy, such as Bhabha electrons or pair-produced muons. Analysis of Mark II data leads to the expression

$$
\frac{\delta_{p}}{p}=\left[(.01 p)^{2}+(.025)^{2}\right]^{\frac{1}{2}}
$$

where $p, p_{\perp}$ are in $\mathrm{GeV}$, and the error on the measured polar angle of the track has been neglected.

During an extended period of data-taking the central drift chamber drew very large currents when operated at nominal high voltages. To mitigate this problem, the high voltages were reduced by about $15 \%$, leading unfortunately to significantly poorer performance, especially for tracks passing very near sense wires. Often the passage of a charged particle failed to produce a signal above threshold on the sense wire, decreasing the number of hits associated with the particle. This in turn led to decreased efficiency both for triggering and for track reconstruction. Eventually, this problem was solved by introducing a small quantity of oxygen into the drift chamber 
gas mixture(originally argon/ethane in equal proportions). It is believed that the oxygen, which is electronegative, damped to a large degree the free electron currents associated with full voltages. See ref. 50 for a more detailed account of this problem and its resolution.

Because this analysis is quite sensitive to uncertainties in trigger efficiency, only the data taken after the introduction of oxygen is used. As will be seen in chapter 5 , inclusion of the data with degraded drift chamber performance would in principle improve sensitivity to slightly heavier lepton masses, but would do little to improve sensitivity to smaller mass differences between the charged and neutral leptons. $\cdots$

\subsection{Time-of-Flight Counters}

The time required for a charged particle to traverse its helical path from the interaction point to the coil radius is measured with 48 plastic scintillators, each subtending about $7 \frac{1}{2}^{\circ}$ in azimuth, with lengths of $3.4 \mathrm{~m}$. Light emitted by the scintillator is detected by photomultiplier tubes mounted at each end. Knowledge of the transit time and of the momentum from drift chamber measurement allows calculation of a particle's mass and therefore provides particle identification. In practice, though, this identification is reliable for only very-low-momentum particles.

In this analysis, relatively loose requirements are imposed on the times measured for detected charged particles, primarily to eliminate obvious cosmic rays, which in general do not enter the scintillators at a sensible time relative to the known time of beam collision. A more important consideration, however, is that TOF hits are required by the event charged track trigger. Since overall efficiency depends critically upon the trigger efficiency, one must understand limitations due to TOF acceptance. For example, between cach TOF counter, there is an insensitive region where the efficiency for detecting the passage of a charged particle is reduced. Figure 4.3 indicates the degraded region in azimuth. In addition, there is a slightly reduced efficiency for triggering on low-momentum hadrons, which is mainly due to two effects. The first is 
that a small fraction of the hadrons decay in flight inside the drift chamber, leading in some cases to badly kinked tracks that are not extrapolated to the correct TOF counter. The second effect occurs at the ends of the TOF scintillators, where lead plates of the end cap calorimeter lie in a hadron's path. A small fraction of the time the particles interact hadronically in one of the plates, preventing their reaching the TOF counter. Because some of these TOF inefficiencies are not accurately simulated by the Monte Carlo, corrections must be applied, as will be discussed in chapter 5 .

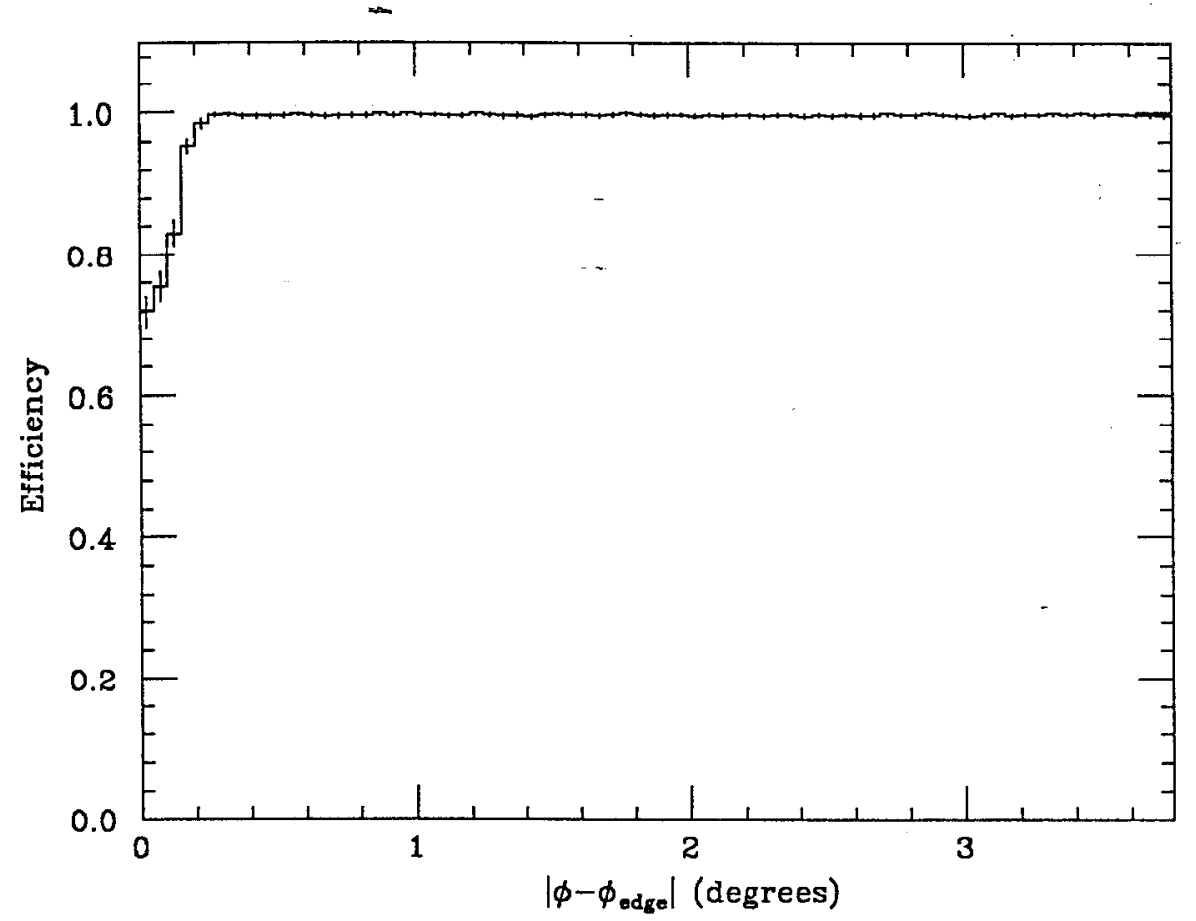

Figure 4.3. The efficiency with which the TOF system detects a Bhabha electron is plotted vs the difference in azimuth between the particle and the nearest TOF counter edge. Detection here is defined as the firing of the time-compensated latch, indicating sensible signals from both photomultiplier tubes at the counter ends. $\Lambda$ veraging these efficiencies over azimuth gives a total efficiency of $98.7 \%$ per track.

\subsection{Magnetic Coil}

The magnetic coil is a double aluminum toroid with hollow aluminum conductors that originally provided a field of 4.7 Tesla. Shortly after the start of data-taking at PEP, an irreparable short developed between the two toroid layers. Thereafter, only 
the outer toroid was powered, providing half the original field, that is, 2.35 Tesla. The reduced field degraded the momentum resolution obtainable with the drift chamber measurements. But for this work, it had the salutary effect of halving the transverse momentum threshold for triggering on a charged track, a consideration important for events with very low visible energy.

\subsection{Electromagnetic Calorimeter}

Electromagnetic calorimetry is provided by eight lead-liquid argon modules arranged in an octagonal barrel surrounding the magnetic coil. Each module is housed in its own cryostat, which leads to large regions of insensitivity between modules. Each module contains 37 planes of $2 \mathrm{~mm}$ thick lead, between which are $3 \mathrm{~mm}$ gaps filled with liquid argon. Alternating lead planes are set at high voltage (typically 3.5 $\mathrm{kV}$ ) and ground. The high voltage planes are divided into strips for measuring the ionization induced in the liquid argon layers. There are 18 planes of lead strips, but in order to rcduce the number of electronics channels, the planes are ganged together to give six "layers." In addition, at the entrance to each module is the so-called massless or trigger gap, where aluminum instead of lead planes are used. Because there is a low probability of that an electromagnetic shower will start in the aluminum, a large energy deposition in this region usually indicates a shower originating in the magnetic coil(1.3 radiation lengths thick at normal incidence). In such a case, a correction for the energy lost in the coil is applied to the observed energy in the calorimeter. The complete ganging pattern for the calorimeter modules is shown in fig. 4.4. There are three different orientations for strips, as shown in fig. 4.5. The $F($ for $\phi)$ strips lie parallel to the beam axis; the $\mathrm{T}($ for $\theta)$ strips lie perpendicular to the beam direction; and the $\mathrm{U}$ strips are oriented at $45^{\circ}$ with respect to both the $\mathrm{F}$ and $\mathrm{T}$ type strips. All three layer types are necessary to distinguish multiple photon showers in the same module.

As mentioned, there are gaps in the azimuth angle between modules; in addition, 


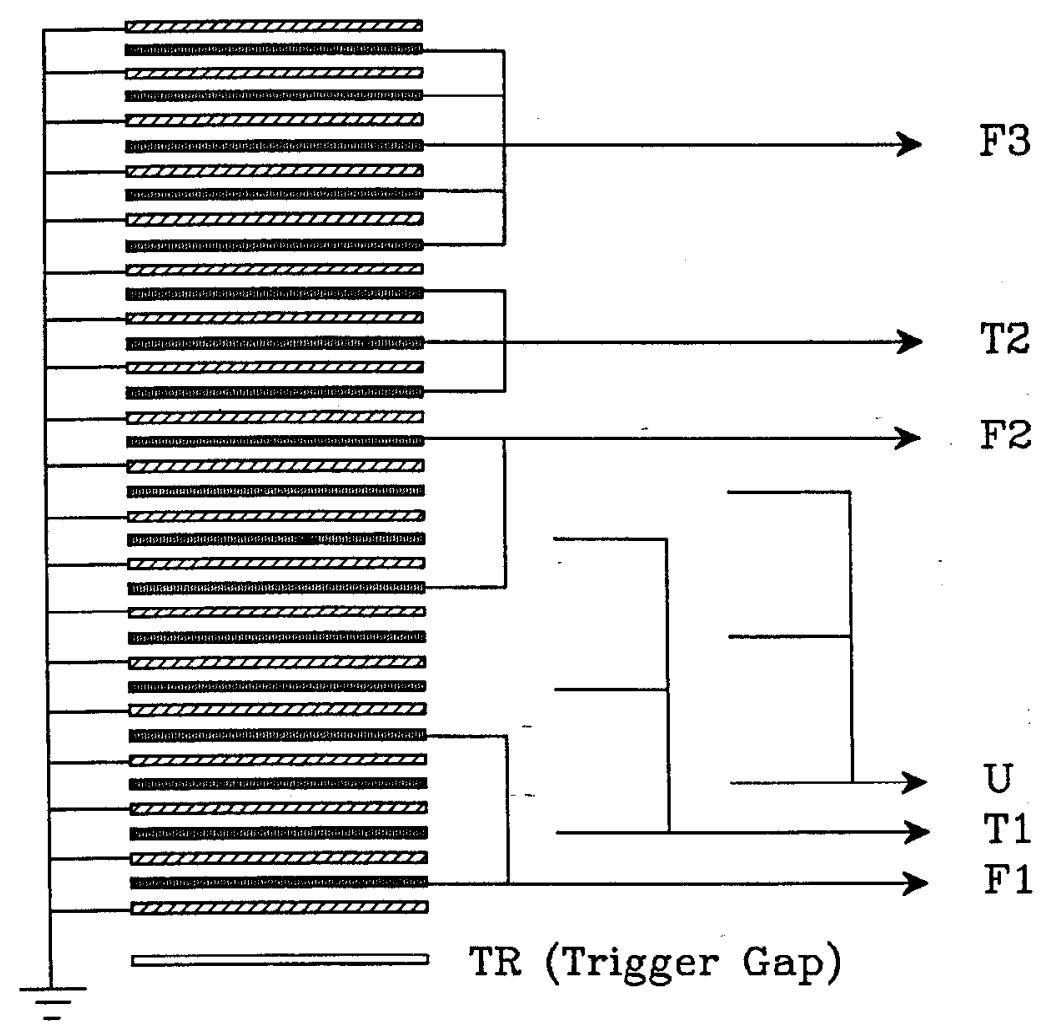

Figure 4.4. Shown is the ganging scheme of electronic channels in the liquid argon calorimeter readout. There are seven distinct layers: four to measure $\phi, \mathrm{TR}, \mathrm{F} 1, \mathrm{~F} 2$, and F3; two to measure theta(approximately), $\mathrm{T} 1$ and $\mathrm{T} 2$; and one at $45^{\circ}, \mathrm{U}$.

the regions within the modules but near the edges have poorer efficiency and energy resolution. This is true in all layer types. Figure 4.6 shows for Bhabha electrons the efficiency for detecting at least $7.25 \mathrm{GeV}$ of energy in the calorimeter vs the difference in azimuth between the electron and the center of the nearest crack between modules. Figure 4.7 shows the same efficiency plotted vs $|\cos \theta|$. To ensure good efficiency and reliable Monte Carlo simulation, conservative cuts are chosen for the tagging photons, as shown in the figures.

Reconstruction of electromagnetic showers is fairly elaborate, involving many parameters that must be determined empirically. A detailed description of the algorithms used can be found in ref. 51. The basic scheme is first to project tracks found from the drift chamber into the calorimeter and to associate any deposited 


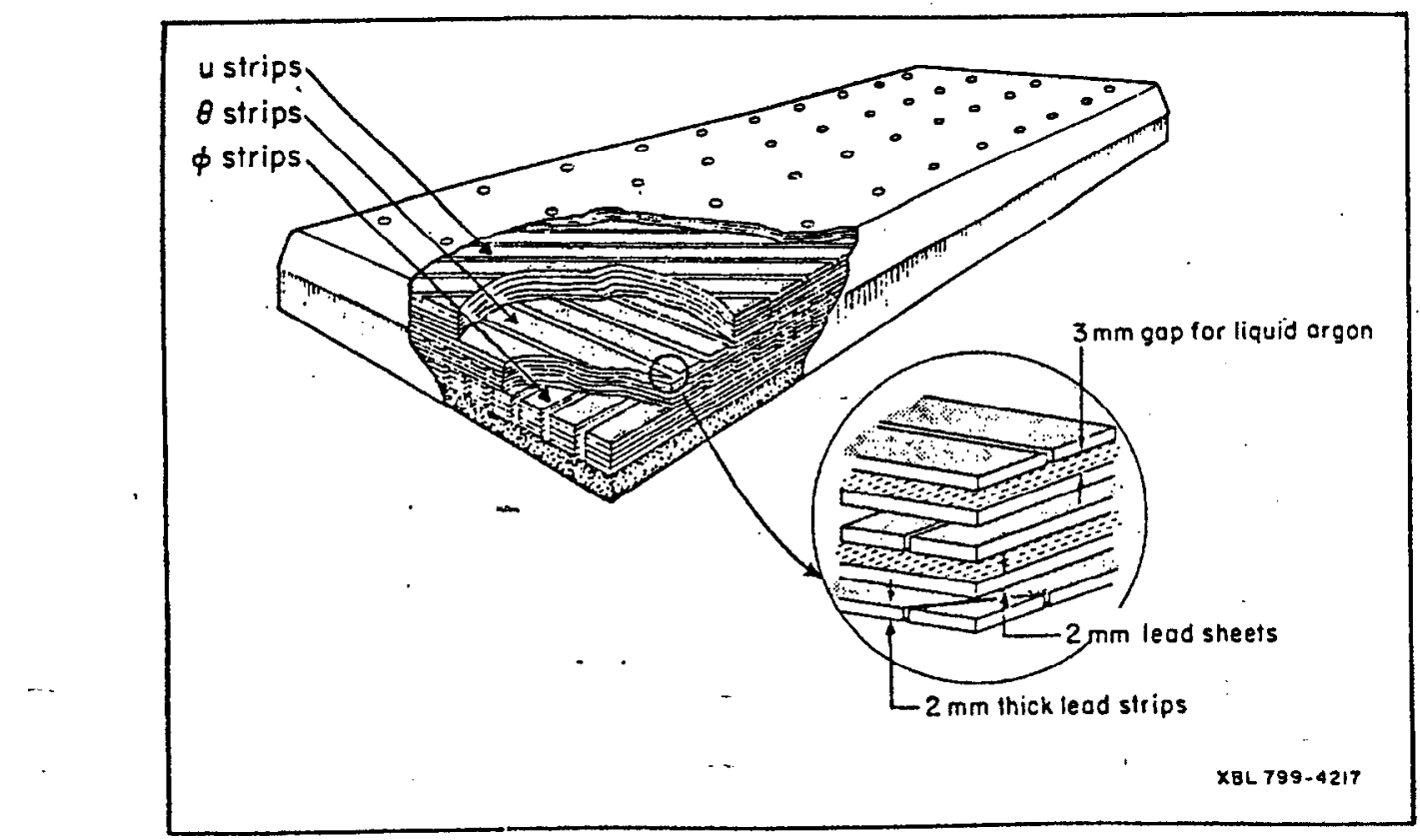

Figure 4.5. Exploded view of liquid argon calorimeter module, showing the three orientations of lead readout strips.

energy in a narrow cone about that projection with the charged track. Next, clusters of strips within each layer are found, based on a threshold energy deposition and contiguity of strips. Neutral shower candidates are then formed from self-consistent combinations of $F, T$, and $U$ strip clusters in the front half of the calorimeter. If geometrically consistent with an existing shower, any energy deposited in the back half of the calorimeter is also added at this point. For showers that share one or more strips in a given layer, algorithms determine how much energy is to be assigned to each shower, based on the energy deposition in all associated layers.

In the Monte Carlo programs, a library of showers created with the $\mathrm{EGS}^{52}$ program allows rapid and fairly accurate simulation of electron and photon interactions in the calorimetcr. Bccause of unsimulatcd inactive rcgions of the calorimetcr, however, an additional smearing of Monte Carlo showers is imposed in this work. The smearing algorithm is based on one applied to high energy Bhabha electron showers, as described in ref. 53. In the algorithm, a fraction of the Monte Carlo showers are 


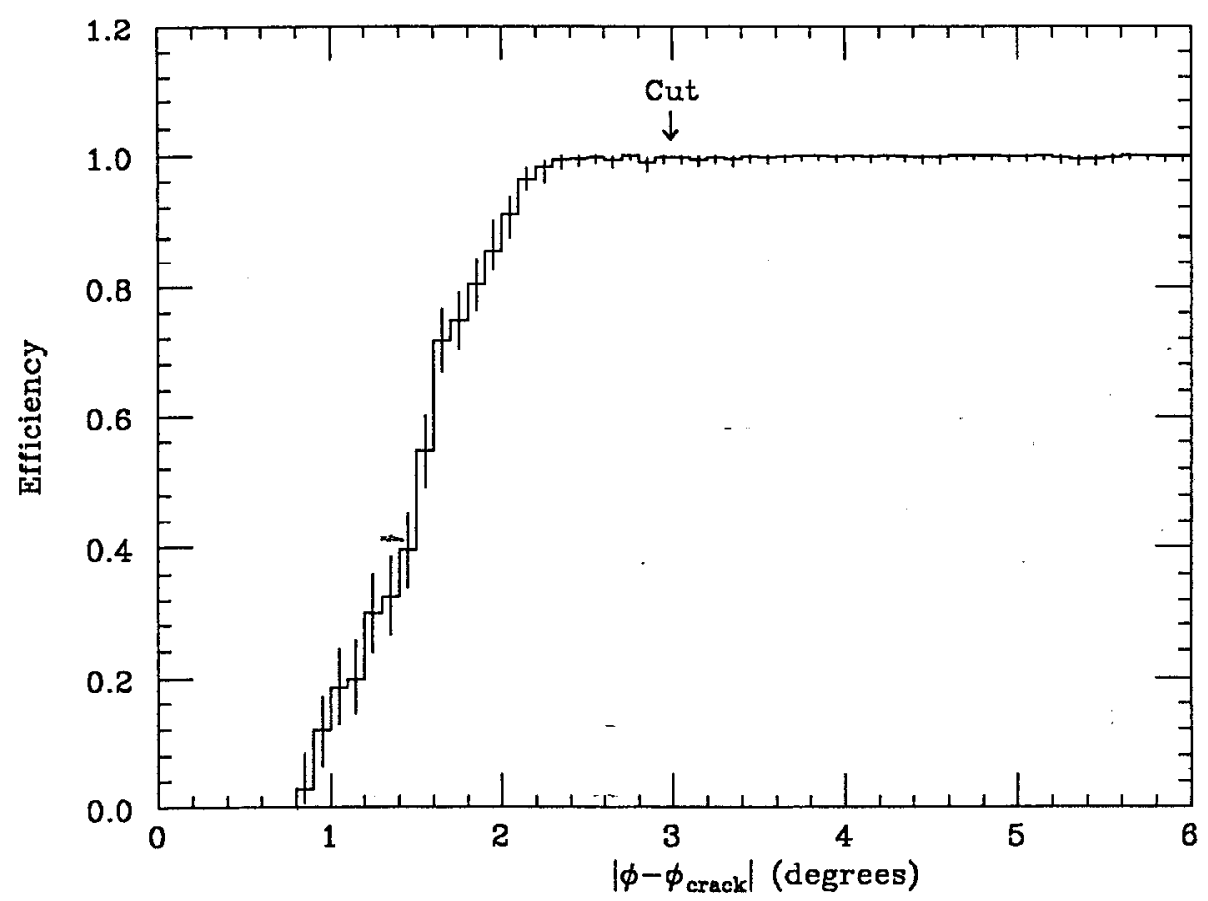

Figure 4.6. Plotted is the detection efficiency (measured energy $\geq 7.25 \mathrm{GeV}$ ) for Bhabha electrons vs the difference in azimuth between the electron and the nearest calorimeter crack. A fiducial cut on $|\cos \theta|$ has already been applied.

smeared according to an exponential decay toward lower energies with a "decay constant" determined from the data. The remaining fraction are smeared according to a Gaussian distribution with $\sigma$ also determined from the data.

Because tagging photon energies are often much lower than that of the Bhabha electrons used in the original study, new values appropriate for low energy showers have been determined. The new values have been derived from low energy electrons produced in the two-photon process $\mathrm{e}^{+} \mathrm{e}^{-} \rightarrow \mathrm{e}^{+} \mathrm{e}^{-} \mathrm{e}^{+} \mathrm{e}^{-}$. The candidate events contain two coplanar charged tracks with nearly equal transverse momenta. Each track must have a total momentum between $800 \mathrm{MeV}$ and $6 \mathrm{GeV}$. It is considered an electron candidate only if the opposite track has an associated energy deposition in the calorimeter at least $75 \%$ of that expected from an electron. Ultimately, for this analysis the efficiency for reconstructing showers produced by photons is desired. Since the shower reconstruction algorithm treats charged and neutral showers differently, 


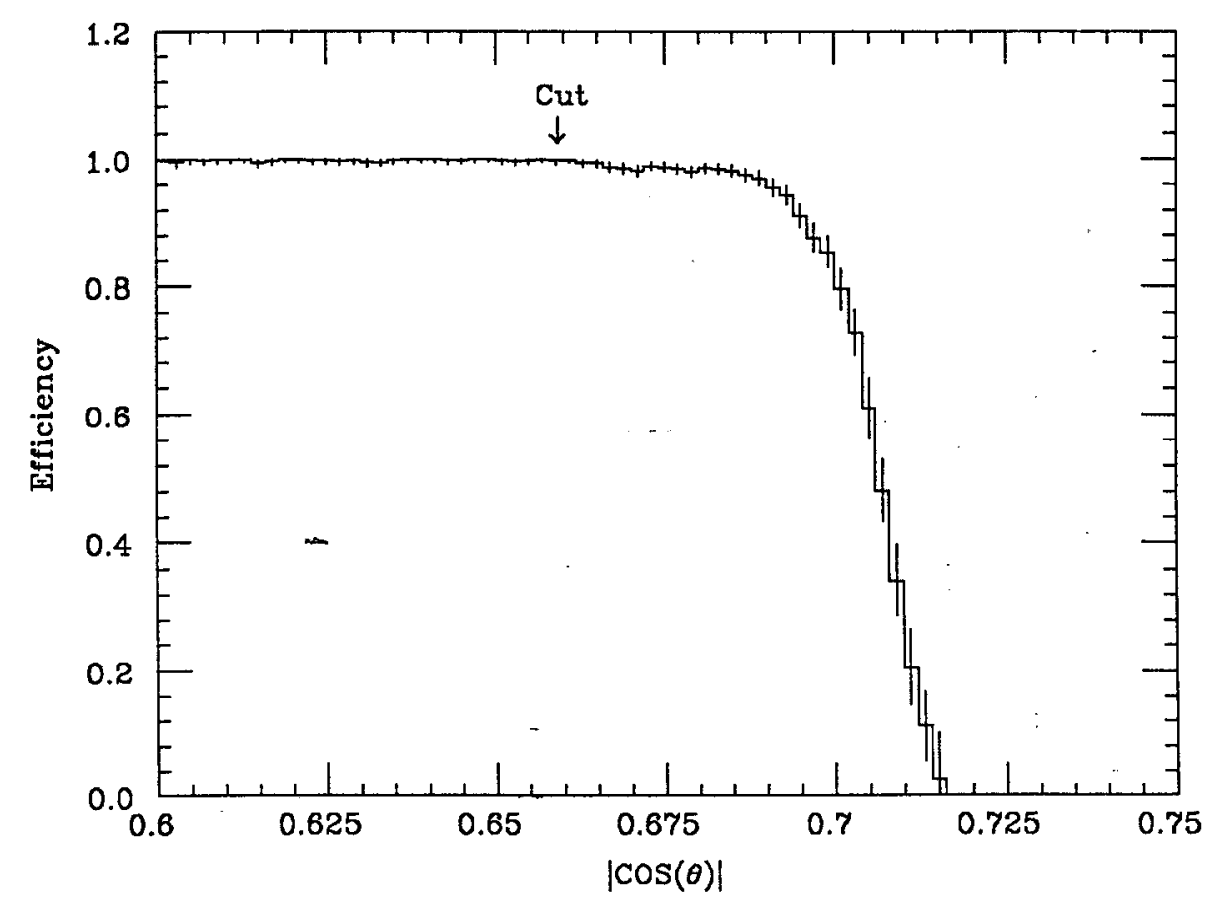

Figure 4.7. Plotted is the detection efficiency (measured energy $\geq 7.25 \mathrm{GeV}$ ) for Bhabha electrons vs the electron's $|\cos \theta|$. A fiducial cut on $\phi$ has already been applied.

in that a specific region is searched for energy clusters, it is necessary in this study to alter the program slightly to ignore the known drift chamber track of the electron. This avoids the reconstruction bias, ensuring the electron shower is treated as a photon shower.

From these electron candidates, a set of smearing parameters has been determined for each $200 \mathrm{MeV}$ interval in measured electron momentum from $800 \mathrm{MeV}$ to $2.0 \mathrm{GeV}$, using the MINUIT fitting program. ${ }^{54}$ For energies higher than $2 \mathrm{GeV}$, where few two-photon produced electrons exist, the parameters determined in ref. 53 are used. In the region studied here, the average fitted fraction smeared with the exponential distribution is found to be $89 \%$, the average decay constant is $4 \%$, and the average fractional gaussian $\sigma$ is $16 \%$. Figure 4.8 shows a small improvement in agreement between data and Monte Carlo with this additional smearing. Plotted is the efficiency for measuring at least $1 \mathrm{GeV}$ energy vs the measured drift chamber momentum of the electron. 


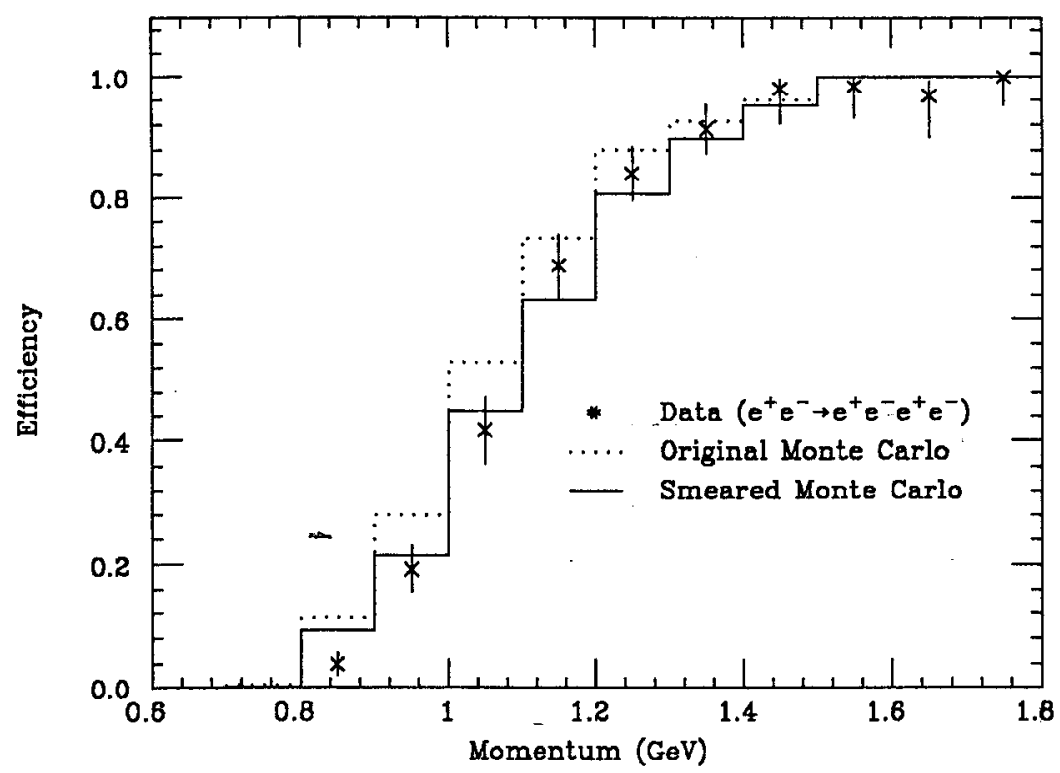

Figure 4.8. Plotted vs the measured electron momentum is the efficiency for measuring $\geq 1 \mathrm{GeV}$ shower energy for original Monte Carlo showers (dotted curve), for smeared Monte Carlo showers (solid curve), and for electrons in the data (points) originating from the process $\mathrm{e}^{+} \mathrm{e}^{-} \rightarrow \mathrm{e}^{+} \mathrm{e}^{-} \mathrm{e}^{+} \mathrm{e}^{-}$.

\subsection{Outer Steel and Proportional Tubes}

Outside of the calorimeter are four walls of muon detectors. Each wall has four layers of steel and four layers of proportional tubes to separate muons from charged hadrons. In order to reach the outermost layer of tubes, a muon must have at least $2 \mathrm{GeV}$ of energy. For incident angles not normal to the plane of tubes, the threshold energy is higher. No cuts based on muon tube hits associated with reconstructed charged tracks are imposed in this analysis. Unassociated muon hits, however, are used to veto certain events induced by cosmic rays, events which will be discussed in chapter 5 .

\subsection{Small Angle Tagger}

At low polar angles sits the Small Angle Tagger(SAT), as shown in fig. 4.9. Three sets of planar drift chambers, each of which has two layers of $\mathrm{x}$ and $\mathrm{y}$ tracking, provide detection of forward charged particles. The chambers are followed by plastic 
scintillators that define different SAT calorimeter acceptances for low-angle Bhabha electrons. Behind the scintillators sits a calorimeter, which consists of a sandwich of lead and plastic scintillators which are read out with $\mathrm{BBQ}^{55}$ wave shift bars. The energy resolution is comparable to that of the barrel calorimeter, with $\delta E / E \approx .15 / \sqrt{E}$ ( $\mathrm{E}$ in $\mathrm{GeV}$ ) for $14.5 \mathrm{GeV}$ Bhabha electrons. ${ }^{53}$ In this analysis, the SAT calorimeters and scintillators are used to veto events that are consistent with hard initial state radiation or two-photon production, as will be described in chapter 5 .

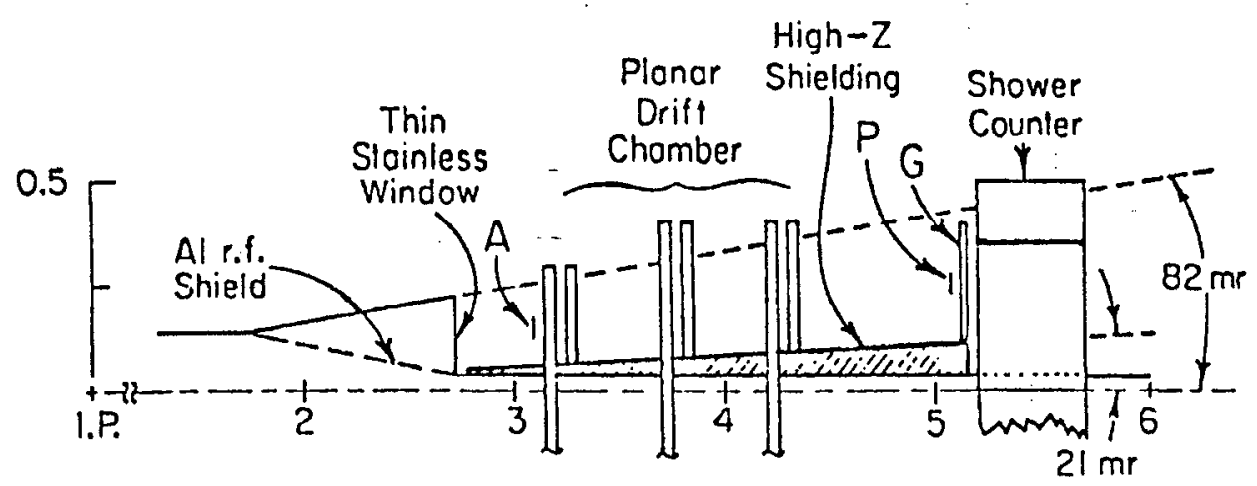

Figure 4.9. Magnified view of small angle tagger. The dimensions shown are in meters. Used in this analysis are the shower counter calorimeter and the plastic scintillator labelled G.

\subsection{Trigger Considerations}

A detailed explanation of the various trigger criteria can be found in ref. 49 . Described here are the requirements important to this analysis. The primary trigger is based on calorimetry information and on drift chamber hits. Designed to be fairly loose, it accepts many events that will eventually be discarded by the secondary trigger. For this analysis, the essential primary trigger is the requirement of hits in various layers of the outer drift chamber. Given the stringent fiducial cuts later placed on the reconstructed tracks, the primary trigger efficiency for this analysis is essentially $100 \%$.

The secondary trigger is much more critical. Although the Mark II detector did take data with a trigger based on calorimetry energy deposition, its efficiency was 
quite poor for photons with energy less than $2 \mathrm{GeV}$. Consequently, it is necessary to rely upon the charged track trigger in this work.

Because rapid readout and large filtering factors are necessary to avoid deadtime in data taking, one must use hardware to identify charged particle candidates. The scheme used by Mark II is based on curvature modules. ${ }^{56}$ These devices are sophisticatcd shift registers that check the pattern of drift chamber hits for consistency with a track left by a charged particle. Each module is designed to look for tracks of a certain range in radius of curvature, corresponding to a certain range in transverse momentum magnitude.

Of the 24 modules, 12 each are devoted to positively and negatively charged tracks. Equally spaced in the inverse of transverse momentum, they were originally designed to be sensitive to transverse momenta above about $130 \mathrm{MeV}$; after the magnet shorted, this threshold dropped to $65 \mathrm{MeV}$. Figure 4.10 shows an idealized drawing of a sample road and the charged track it contains. In order to ensure good efficiency, some overlap in azimuth and in curvature between roads was designed, so that often a charged particle triggers more than one track in the same curvature module and more than one curvature module. In addition, there is some doublecounting in azimuth at the beginning and end of the shift registers scan. As a result, the hardware logic must often combine curvature module firings to find its charged track candidates, called $\mathrm{A}$ tracks. One additional requirement on an $\mathrm{A}$ track is that there be a signal on both ends of the TOF counter associated with the track. (For three of the counters, only one signal is required since their photomultipliers on one end were damaged during the magnet short.) This imposes an automatic fiducial trigger requirement that a charged particle have an angle greater than about $40^{\circ}$ with respect to the beam direction. As mentioned before, cracks in azimuth between TOF scintillators lead to a loss in trigger efficiency.

Under typical running conditions, curvature modules require at least 2 hits in the inner band of $\mathrm{VC}$ sense wires and have fairly tight requirements on the allowed 


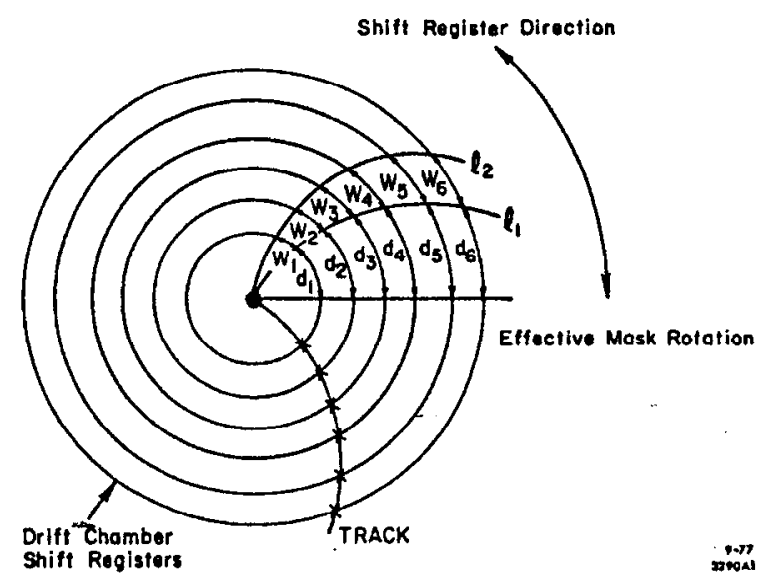

Figure 4.10. Shown is an example of fast charged particle tracking with hardware. The $\operatorname{arcs} \ell_{1}$ and $\ell_{2}$, defined by delays $d_{\mathbf{i}}$ and widths $\mathrm{w}_{\mathrm{i}}$, enclose between them a "road." If enough layers have hits, that is, detected sense wire signals, within the road, the trigger hardware considers there to be a track. The entire drift chamber is systematically searched for such tracks by varying the delays and widths, while clocking the bit pattern hits through shift registers. In practice, the simple road pattern shown here was found inadequate at PEP. To exclude cosmic ray particles and other backgrounds; it was necessary to force the arcs $\ell_{1}$ and $\ell_{2}$ to cross near their centers, splitting the road into two sections.

width of roads. This suggests that particles with large impact parameters, that is, large distances of closest approach to the interaction point in the transverse plane, may be missed by the modules. Because close-mass leptons with very small differences can give rise to reconstructed tracks with large impact parameters, this possible inefficiency has been investigated. A control sample of $\mathrm{K}_{\mathrm{s}}^{0}$ particles produced in twophoton events was used in the study. The charged pions from these $\mathrm{K}_{\mathrm{s}}^{0}$ decays tend to have relatively low transverse momentum, comparable to that expected of closemass lepton decay products, and to have large impact parameters. The candidates are identified with the VFINDP program, ${ }^{57}$ which selects pion pairs on the basis of invariant mass, quality of vertex fit and reconstructed impact parameters. In order to avoid bias, a track is considered in the efficiency study only if its presence is not necessary for the event to have triggered. 
Trigger efficiency is found to depend on impact parameter, but not in a simple way. There is an asymmetry in trigger efficiency for high impact parameter tracks, an asymmetry that depends on which way the track is bent by the magnetic field as it leaves the vicinity of the interaction point. If it is bent toward the beam in the transvcrsc planc, its probability of producing an $\Lambda$ track is reduced.

Figure 4.11 shows a simple example. Two charged tracks of the same transverse momentum magnitude, but with opposite charges, originate from a point $4 \mathrm{~cm}$ to the right of the beam, travelling vertically. Each leaves a trail of drift chamber hits, which are to be contained in a curvature module road that assumes the track originated from the origin. For each track, such a road of fairly narrow width can be found that contains all hits, but what is important is that the left-hand road has a smaller average radius of curvature than the right-hand road. In fact, in this example, the left-hand radius of curvature corresponds to a transverse momentum below the threshold of the curvature modules, while the right-hand track exceeds that threshold. In other words, the right-hand track would be detected by the hardware, but the left track would not. In this instance, it is the negatively charged track that would be missed. If the particles started $4 \mathrm{~cm}$ to the left of the beam, with the same initial directions, the positively charged track would be missed. As one would expect, the asymmetry averages out over particle charge and over location of the point of closest approach to the beam, but the asymmetry does introduce an overall efficiency loss.

Empirically, it is found that this effect correlates well with the variable $X_{a}$, defined by

$$
X_{a} \equiv\left(\hat{p}_{\perp} \times \overrightarrow{\mathrm{r}}_{\min }\right)_{\mathbf{z}} \frac{\mathrm{Q}}{\sqrt{p_{\perp}}}
$$

where $\mathrm{Q}$ is the track's charge, and $\hat{p}_{\perp}$ is the transverse direction of the track at its closest approach to the beam in the transverse $(x-y)$ plane. The vector $\vec{r}_{\min }$ is the transverse component of the vector from the beam center to that point of closest approach. The efficiency for producing an A track trigger, as determined from the 

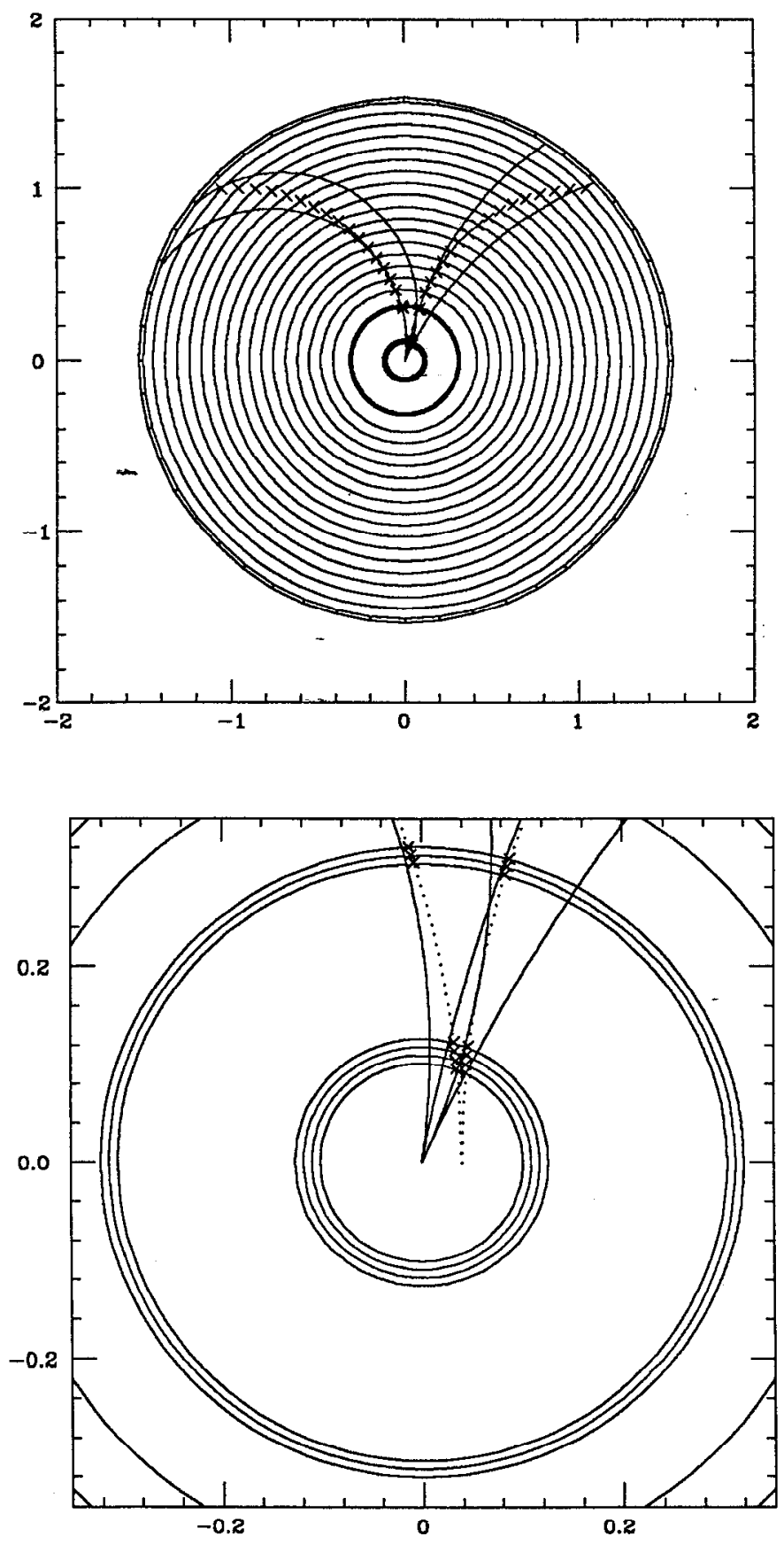

Figure 4.11. Shown on top are two tracks of equal transverse momenta, but of opposite charges. The track that bends to the left is less likely to be detected by the trigger hardware than the one that bends right. The bottom figure shows a magnification of the inner layer region. For simplicity, the arcs defining the depicted roads do not cross. 
sample of $\mathrm{K}_{\mathrm{s}}^{0}$ candidate tracks, is plotted vs $X_{a}$ in fig. 4.12 , along with a simple fit to the data points. Also shown are conservative cuts imposed on charged track candidates used in this analysis. There are virtually no $\mathrm{K}_{\mathrm{s}}^{0}$ candidate tracks with impact parameters greater than $5 \mathrm{~cm}$ in the transverse plane, precluding an easy direct measurement of the efficiency for very large impact parameter tracks from the data. Therefore, a conservative requirement-imposed in this analysis is that no candidate charged tracks have such a large impact parameter.

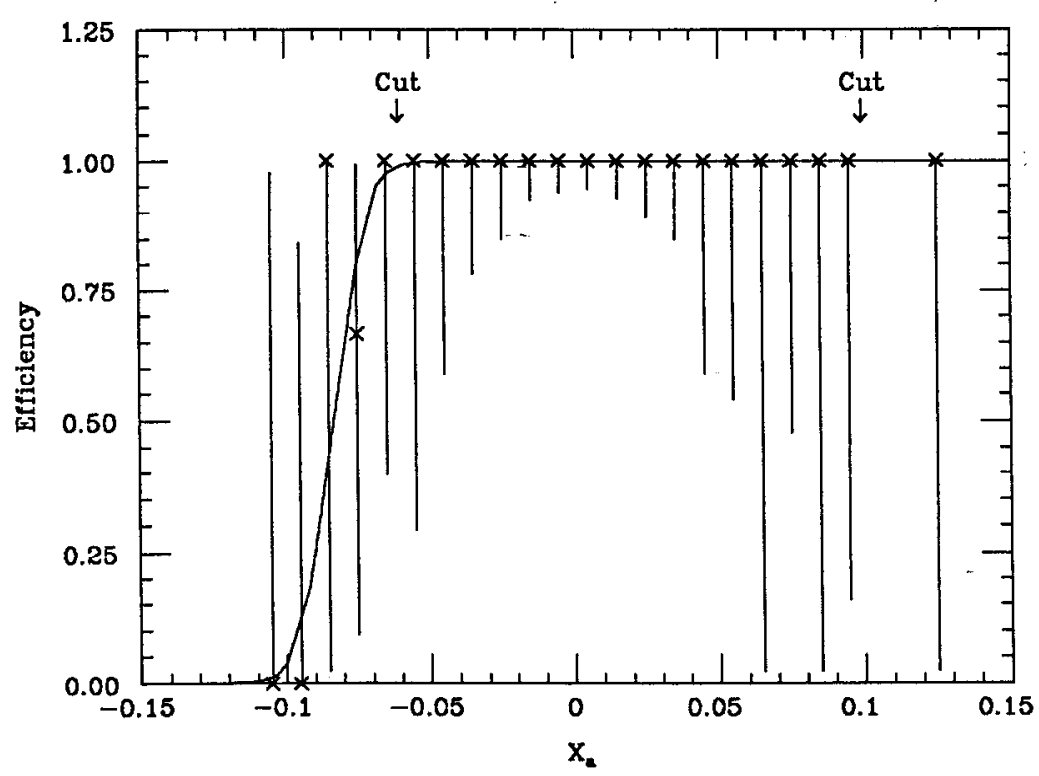

Figure 4.12. Plotted is the efficiency for the trigger hardware properly detecting a charged track originating from $\mathrm{K}_{\mathrm{s}}^{0}$ decay vs the variable $X_{a}$, defined in the text. 


\section{Chapter 5}

\section{Analysis and Results}

This chapter describes in detail the analysis procedure and presents final results. Given first are the event selection criteria designed to distinguish a lepton signal from conventional backgrounds. Estimates of remaining contamination from conventional background processes are derived from Monte Carlo studies. These estimates include explicit corrections for inaccuracies in simulating the Mark II detector. After these corrections are applied, the background estimates are compared to what is observed in the data and the agreement evaluated. From the comparison and from further Monte Carlo study of expected signal rates, limits on close-mass leptons are derived. Finally, a summary of these results is given, and related, future searches at higher energies are discussed.

For.the purpose of illustration, three examples of close-mass leptons have been singled out. In many of the figures given in this chapter, distributions in certain variables will be presented for these three cases and compared with those expected from one or more backgrounds. Each case corresponds to a lepton mass difference of $400 \mathrm{MeV}$. The three examples are labelled $\mathrm{A}$ for $\left(\mathrm{M}_{\mathrm{L}}=2 \mathrm{GeV}, \mathrm{M}_{\nu_{\mathrm{L}}}=1.6 \mathrm{GeV}\right)$; $B$ for $\left(M_{L}=6 \mathrm{GeV}, M_{\nu_{L}}=5.6 \mathrm{GeV}\right)$; and $\mathrm{C}$ for $\left(\mathrm{M}_{\mathrm{L}}=10 \mathrm{GeV}, \mathrm{M}_{\nu_{\mathrm{L}}}=9.6 \mathrm{GeV}\right)$.

\subsection{Event Selection}

\subsubsection{Charged track requirements}

Since the aim of this work is to explore very low mass differences, where multi- 
particle decays are forbidden or highly suppressed by the available phase space, one can safely require there be only two charged particles in an event. This condition incurs a tiny loss in efficiency and greatly simplifies subsequent analysis. Therefore, the first requirement imposed is that there be exactly two charged tracks reconstructed in the central drift chambers. In order to ensure very high trigger efficiency and thus reduce dependence upon unreliable Monte Carlo trigger simulation, each track must also satisfy fairly stringent requirements:

1) The track momentum must make an angle greater than $45^{\circ}$ with respect to either beam direction.

2) The momentum transverse to the beam $\left(p_{\perp}\right)$ must be greater than $150 \mathrm{MeV}$.

3) There must be a signal from both photomultiplier tubes of the TOF counter in the track's projected path. In addition, the measured flight time must be in the range $0-12$ ns.

4) The track must have at least 10 associated drift chamber signals (out of a possible 23 ), and at least one of those signals must come from one of the four inner layers of the vertex chamber.

5) The $\chi^{2}$ per degree of freedom calculated from the helical track fit to the drift chamber signals must be less than 5 .

6) As described in chapter 4, the impact parameter of the track in the transverse plane with respect to the beam collision point must be less than $5 \mathrm{~cm}$, and the variable $X_{a}$ must be in the range from -.06 to +.10 .

In addition to these largely fiducial and track quality requirements, the tracks must have a total measured momentum less than $4 \mathrm{GeV}$, and any liquid argon calorimeter energy associated with the charged track must also be less than $4 \mathrm{GeV}$. These requirements tend to select events with low visible energy, filtering out backgrounds from radiative tau pair production and mismeasured radiative Bhabha events. Figure 5.1. shows the distribution in the reconstructed momenta ( 2 entries/event) for the radiative tau background and for the three sample heavy lepton cases. 


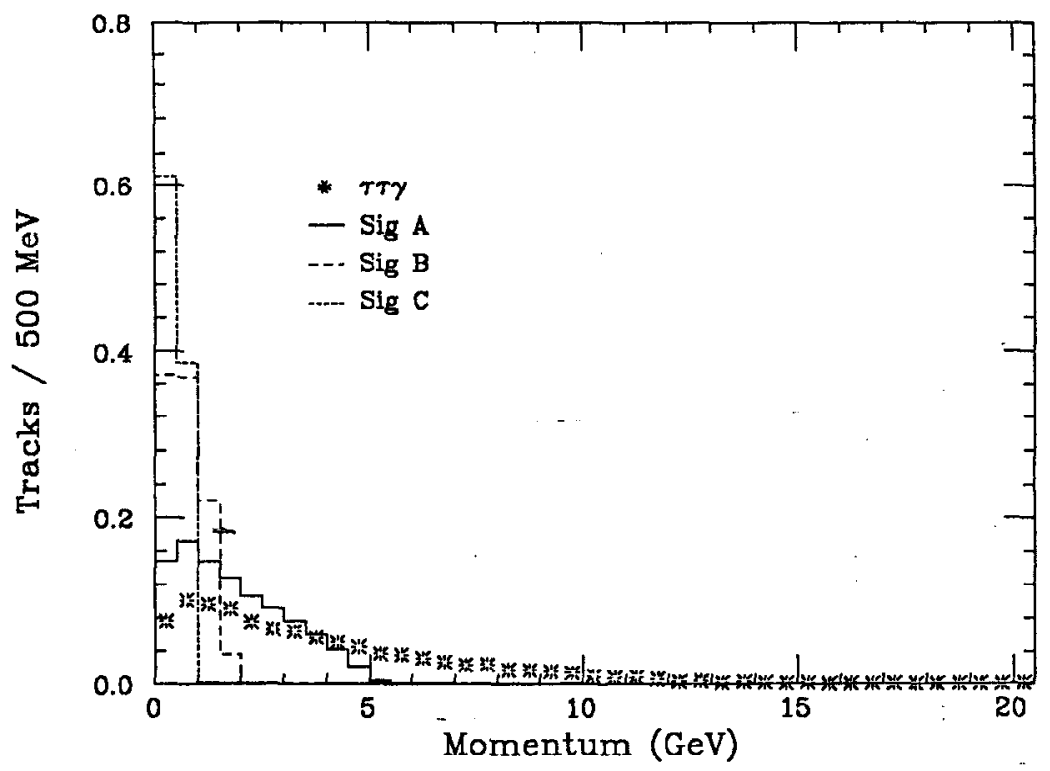

Figure 5.1. Distribution in reconstructed charged track momenta ( 2 entries/event) for the radiative tau background and for the exemplary signals $\mathrm{A}, \mathrm{B}$, and $\mathrm{C}$.

\subsubsection{Tagging photon requirements}

To reduce backgrounds from $\pi^{0}$ decays, the tagging photon must be energetic and isolated both from other neutrals and from the charged tracks. In addition, to ensure good efficiency and, more important, to ensure accurate Monte Carlo simulation of calorimetry response, the photon must satisfy stringent fiducial conditions. The criteria imposed are the following:

1) The measured energy must be at least $1 \mathrm{GeV}$

2) The photon polar angle $\theta_{\gamma}$ with respect to the beam must satisfy $\left|\cos \theta_{\gamma}\right| \leq 0.66$.

3) The photon's azimuth direction must be at least $3^{\circ}$ away from the center of the nearest crack between calorimeter modules.

4) The total reconstructed energy deposition in the calorimeter within $30^{\circ}$ of the photon must be less than $150 \mathrm{MeV}$. 
5) No reconstructed neutral in the calorimeter within $90^{\circ}$ of the photon can combine with it to give an invariant mass consistent with a $\pi^{0}$ or $\eta$, where consistent means

$$
\left|\mathrm{M}_{\gamma_{1} \gamma_{2}}-\mathrm{M}_{\pi^{0}, \eta}\right| \leq \delta \mathrm{M}
$$

and where for the $\eta$ condition, the other photon must have at least $150 \mathrm{MeV}$ energy. The mass error is approximated by

$$
\delta \mathrm{M}(\mathrm{GeV}) \approx \frac{1}{2}(0.15) \sqrt{\frac{1}{\mathrm{E}_{\gamma_{1}}}+\frac{1}{\mathrm{E}_{\gamma_{2}}}} \mathrm{M}_{\gamma_{1} \gamma_{2}}
$$

6) The angle between the photon and nearest charged track momentum must be at least $45^{\circ}$.

In the case that more than one photon in an event satisfies the tagging requirements, the photon with the least total nearby (within $30^{\circ}$ ) neutral calorimetry energy is taken as the tag. If more than one has no nearby neutral energy, the most cncrgctic is taken as the tag. Figure 5.2. shows the distributions in nearby energy for the radiative tau background and for the exemplary heavy lepton signals.

\subsubsection{Event topology requirements}

At this stage in the analysis, additional requirements, designed to suppress various backgrounds, are imposed on candidate events. Because charged tracks close to one another may induce a signal on the same drift chamber wire, or on wires close enough to create confusion during track reconstruction, cuts are imposed on the opening angle between the two charged tracks. The opening angle must be at least 100 mrad in the transverse plane. In addition, the opening angle in 3 dimensions must be at least 20 degrees; this latter requirement suppresses not only events with unreliable tracking, but also the substantial background from inital state radiation in tau pair production and hadronic events, where the photon has nearly the beam energy. 


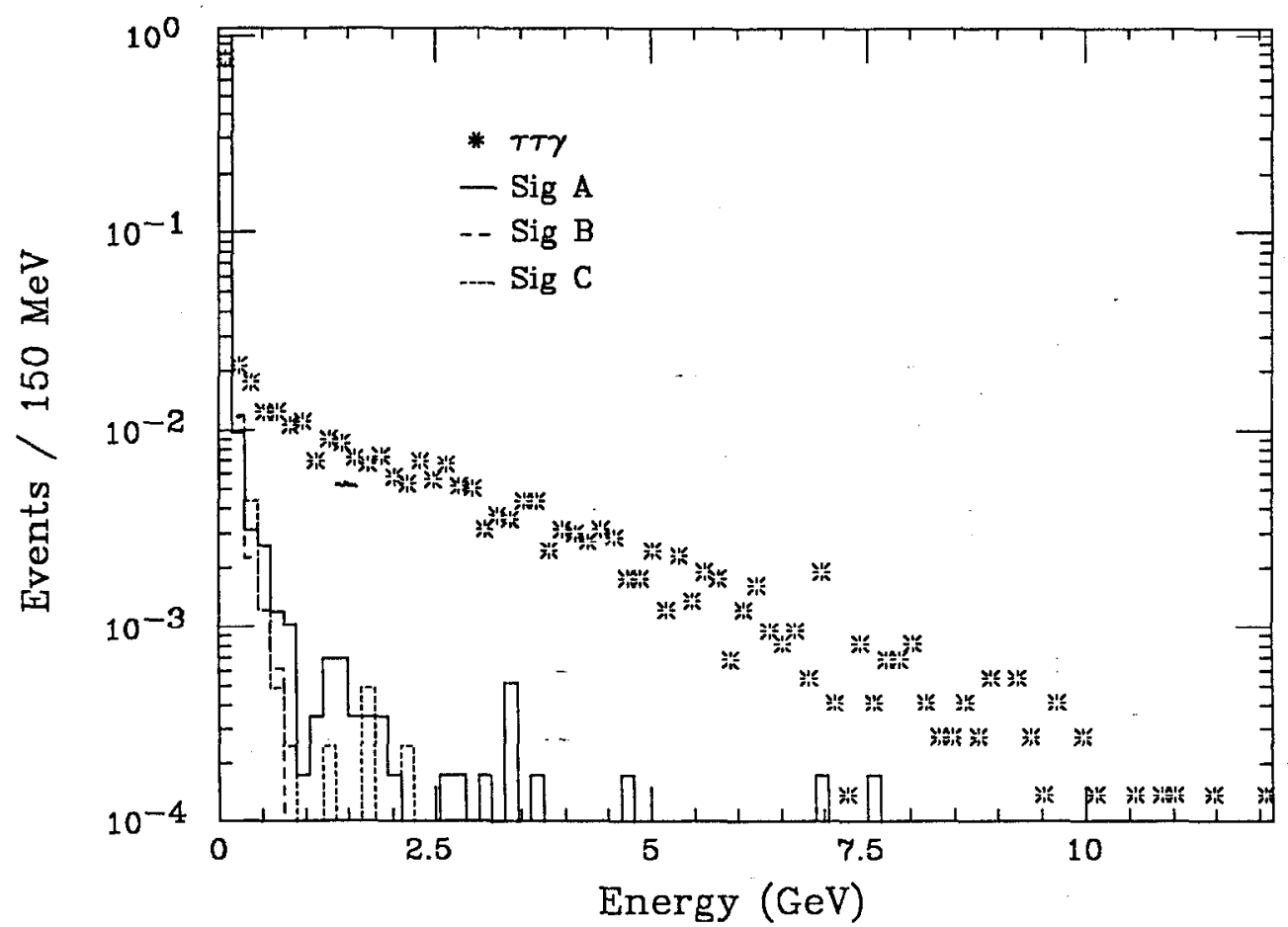

Figure 5.2. Distributions in total neutral energy near(within $30^{\circ}$ ) to the tagging photon for the radiative tau background and for the exemplary signals $\mathrm{A}, \mathrm{B}$, and $\mathrm{C}$.

Upper limits too are placed on the 2- and 3-dimensional opening angles between the charged tracks. For reasons discussed in the next section, events can occur where two charged particles are produced through a two-photon process, while an unrelated shower is measured in the calorimetry. In such a case, the two tracks will in general be coplanar, that is, they will appear back-to-back in the transverse plane, although their longitudinal components along the beam direction may be quite different. This arises because, as described in chapter 1, two-photon processes have an enormous cross section when the electron and positron are only slightly deflected from their original directions. This implies that they do not carry much transverse momentum, so that particles visible in the central detector must to a good approximation conserve transverse momentum. Consequently, one can cut on the net measured transverse momentum of all detected particles, requiring it be greater than a certain value in 
order to suppress two-photon processes. In the case of two charged particles, however, a better variable is the angle between the charged particles in the transverse plane, since particle directions are better-measured than momentum magnitudes. In this analysis, the two charged particles must have an acoplanarity angle(deviation from $\pi$ of transverse opening angle) greater than 20 mrad. In order to suppress further the backgrounds from radiative tau production and badly mismeasured radiative Bhabha events, the opening angle between the charged tracks in three dimensions must be no greater than $160^{\circ}$. The distribution in this angle is shown in fig. 5.3 for the radiative tau background and for the exemplary signal cases.

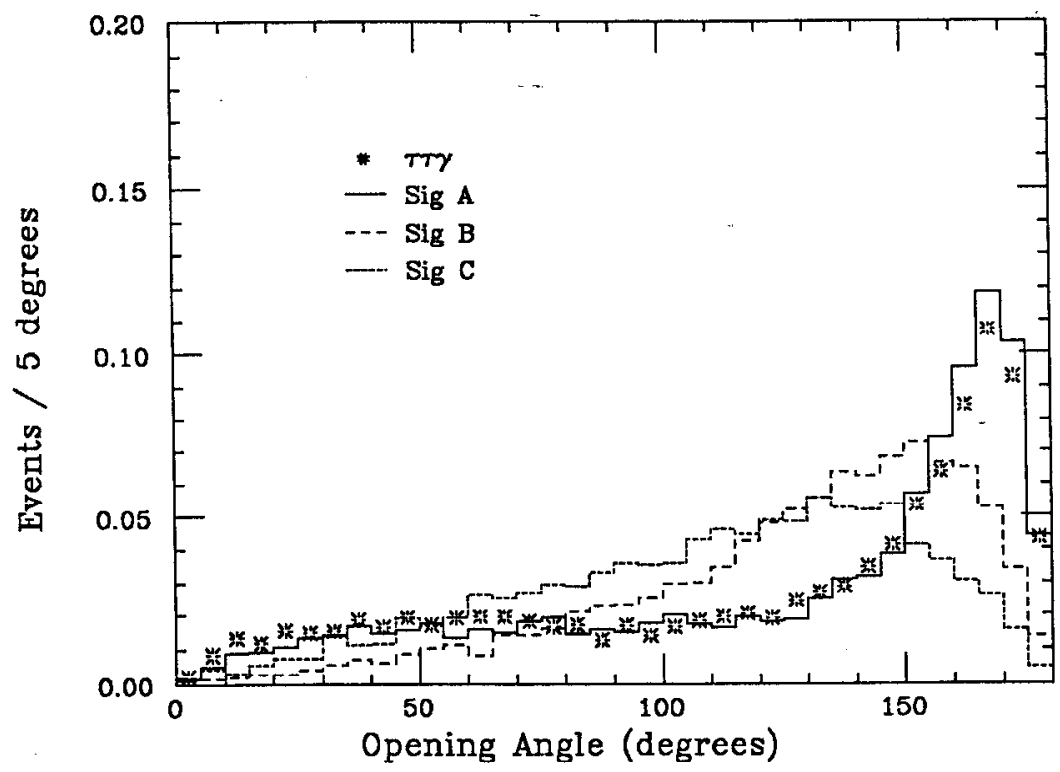

Figure 5.3. Distribution in the opening angles between charged tracks for radiative tau background and signal examples $\mathrm{A}, \mathrm{B}$, and C.

In order to suppress radiative tau pair production accompanied by decays involving one or more $\pi^{0} \mathrm{~s}$, the total neutral energy of the event, excluding the contribution from the tagging photon, must be less than $2 \mathrm{GeV}$. The distribution in energy is plotted in fig. 5.4 for radiative tau events and for the exemplary signal cases.

The electron and positron in a two-photon process can carry away considerable longitudinal momentum along the beam direction, without absorbing much transverse 


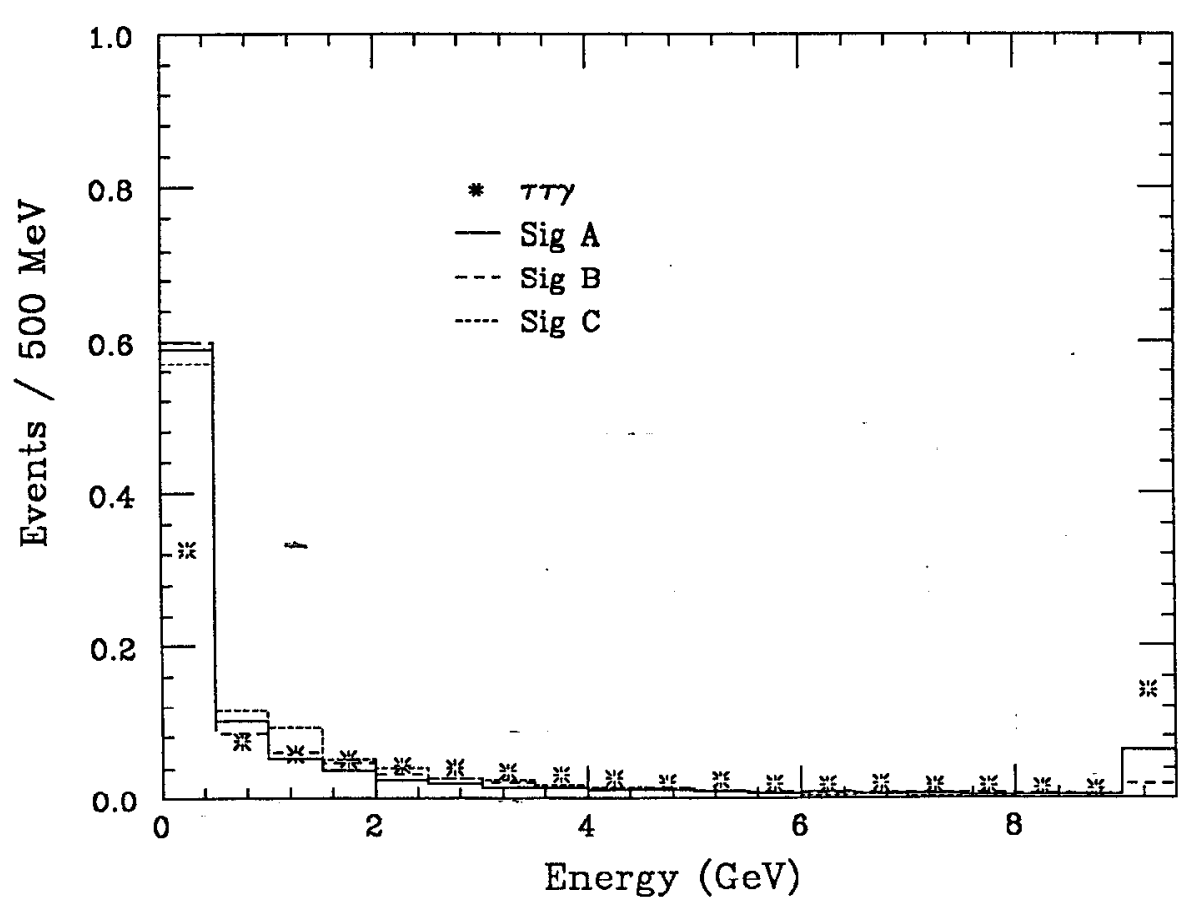

Figure 5.4. Distribution in total neutral energy detected in the liquid argon calorimeter, excluding the tagging photon, for radiative tau background and for signal cases $\mathrm{A}, \mathrm{B}$, and $\mathrm{C}$.

momentum. From momentum conservation, this means the detected particles taken together can also have a longitudinal momentum much greater than their transverse component. To reduce such backgrounds, it is required that the transverse component be greater than the longitudinal component, that is, the direction of the net detected momentum must make an angle greater than $45^{\circ}$ with respect to the beam. This requirement suppresses not only two-photon backgrounds, but also events with hard initial state radiation along the beam direction.

Similarly, the total momentum of the tagging photon, the two charged tracks, and any additional neutral showers in the calorimeter is required to have a transverse component greater than $1 \mathrm{GeV}$. Note that in many cases, it is the tagging photon that contributes most to the transverse momentum, since the charged particles often have low momenta.

This last cut removes nearly all of the remaining conventional two-photon back- 
grounds, since two-photon backgrounds fall off very rapidly with transverse momentum. An exception to this general rule occurs when a particle with large transverse momentum goes undetected. For example, photons that miss the active regions of the calorimetry can come from $\pi^{0}$ decays in the process

$$
\begin{aligned}
& \mathrm{e}^{+} \mathrm{e}^{-} \rightarrow \mathrm{e}^{+} \mathrm{e}^{-} \rho^{+} \rho^{-} \\
& \rho^{+} \rightarrow \pi^{+}\left[\pi^{0} \rightarrow \gamma \gamma\right] \\
& \rho^{-} \rightarrow \pi^{-}\left[\pi^{0} \rightarrow \gamma \gamma\right]
\end{aligned}
$$

In addition, one pair of pions can come from a charged rho and the other from continuum production, or all four pions can come from continuum production. Typically, the most energetic detected photon will tag the event, and another will escape through either a crack in azimuth between liquid argon modules or at a forward angle low enough to miss the liquid argon, but high enough to carry away substantial transverse momentum. Ideally, some of these events could be identified from information in the end cap calorimeters, but as discussed in the previous chapter, the poor resolution and efficiency of the end caps make them unsuitable. To help remove such events, it is required that the azimuth of the event's missing momentum be at least $3^{\circ}$ away from the center of the nearest crack between liquid argon modules, the same cut imposed earlier on the tagging photon's azimuth.

\subsubsection{Event veto conditions}

In addition to the selection cuts described so far, there are other conditions in the detector that may indicate background events. One of these is energy deposited in the SAT calorimeter. Large measured SAT energy may be due to a deflected beam electron, suggesting a large q-squared two-photon process(the probability of two different electron-positron interactions occurring during the same beam crossing is negligible). Such events cause concern because an electron deflected by a large angle can carry away enough transverse momentum to ensure that the remaining particles seen in the 
central detector pass the $1 \mathrm{GeV}$ cut on missing transverse momentum. Unfortunately, there is a significant background due to photons from synchrotron radiation induced by the bending magnets of the PEP ring. Figure 5.5. shows the distribution in total measured SAT energy for events where two back-to-back high-energy electrons are detected by the central drift chamber and liquid argon calorimeter. This detected energy is mainly a measure of backgrounds- due to synchrotron radiation, although other beam-related backgrounds and initial state radiation contribute also. From this distribution, it is clear that demanding the total energy be very small would incur unacceptable loss in efficiency. For this analysis, a cut is made at $8 \mathrm{GeV}$, leading to an inefficiency of $0.7 \%$.

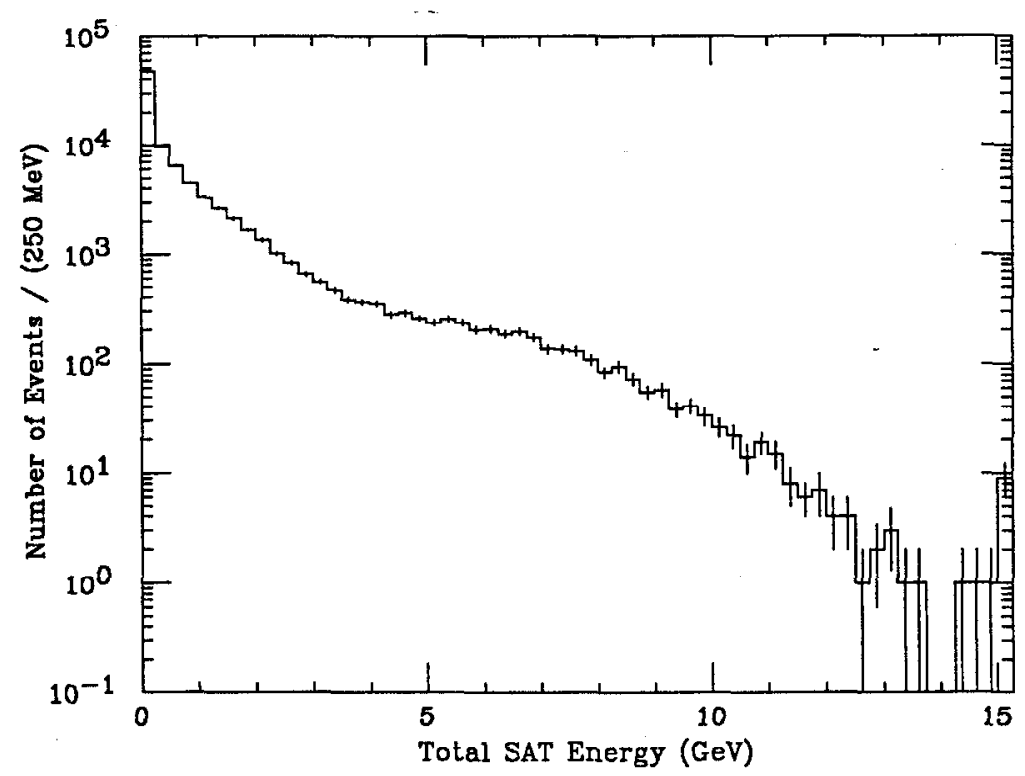

Figure 5.5. Distribution in total measured SAT energy for Bhabha events where the electrons are tagged in the central detector. This is mainly a measure of noise due to beam-induced backgrounds.

Additional SAT information comes from a scintillator called the gross defining counter, placed in front of each calorimeter. The scintillator produces an electronic latch if it detects the passage of a charged particle. By requiring the presence of a scintillator latch, one can again examine SAT energy deposition, with the hope of distinguishing backgrounds due to synchrotron radiation and other noise from that due 
to deflected electrons and positrons. Figure 5.6 shows the distribution in SAT energy for central detector Bhabha events when a signal from the gross defining counter is present. Clearly, the backgrounds are severely suppressed by the coincidence requirement, allowing a cut at a measured energy as low as $200 \mathrm{MeV}$, with only a $3.9 \%$ loss in signal efficiency.

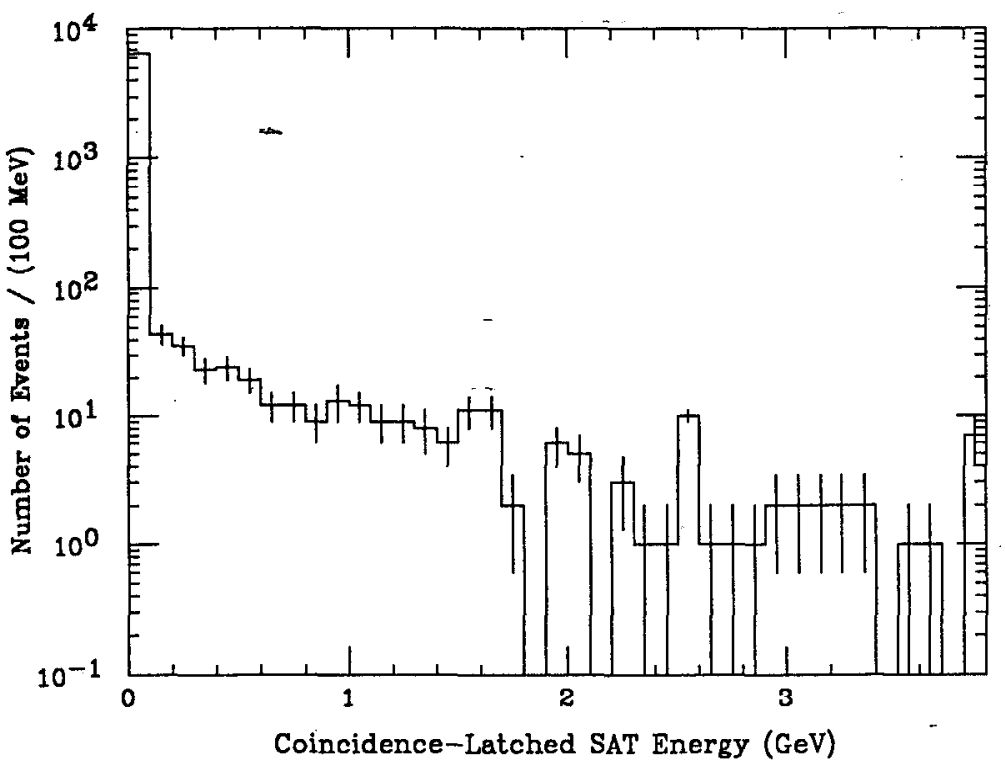

Figure 5.6. Distribution in SAT energy for Bhabha events where the electrons are tagged in the central detector and where there is a simultaneous signal in the gross defining counter. This is a a measure of backgrounds due to beam electrons scattered into the SAT calorimeter system.

Another background, quite peculiar to this analysis, is that of a cosmic ray muon inducing an electromagnetic shower in the liquid argon calorimeter, in coincidence with a electron-positron interaction that produces two charged particles in the detector. The cosmic ray shower is reconstructed and misinterpreted as a photon by conventional analysis programs because, usually, there is no charged track leading from the beam collision point to the shower region. Figure 5.7 shows a dramatic example. The signals in the left outer layers of the muon layers indicate the entrance of a cosmic ray muon that then produces a shower in the calorimeter through collision with an electron in the calorimeter material. The "knock-on" electron quickly 
bremsstrahlungs in the calorimeter, initiating the detected shower. In this event, not all of the shower is contained by the calorimeter; some high energy electrons penetrate to the drift chamber region leaving clear tracks. Because the standard event reconstruction software has been optimized to identify charged particles originating from the beam collision point, these stray electrons from the shower arc missed. Extrapolating along the muon direction, one can see the straight trail of drift chamber signals it leaves behind, a small energy deposition in the bottom right calorimeter module due to ionization, and some additional signals in the right muon wall. At the same time, two low momentum particles are produced by an electron-positron interaction at the beam collision point.

$$
\begin{aligned}
& \text { RUN } 11388 \text { REC } 4854 E=29.00 \quad \text { 2 PRONG OTHER E-E } \\
& \text { TRIGGER } 04 \mathrm{~A} S
\end{aligned}
$$

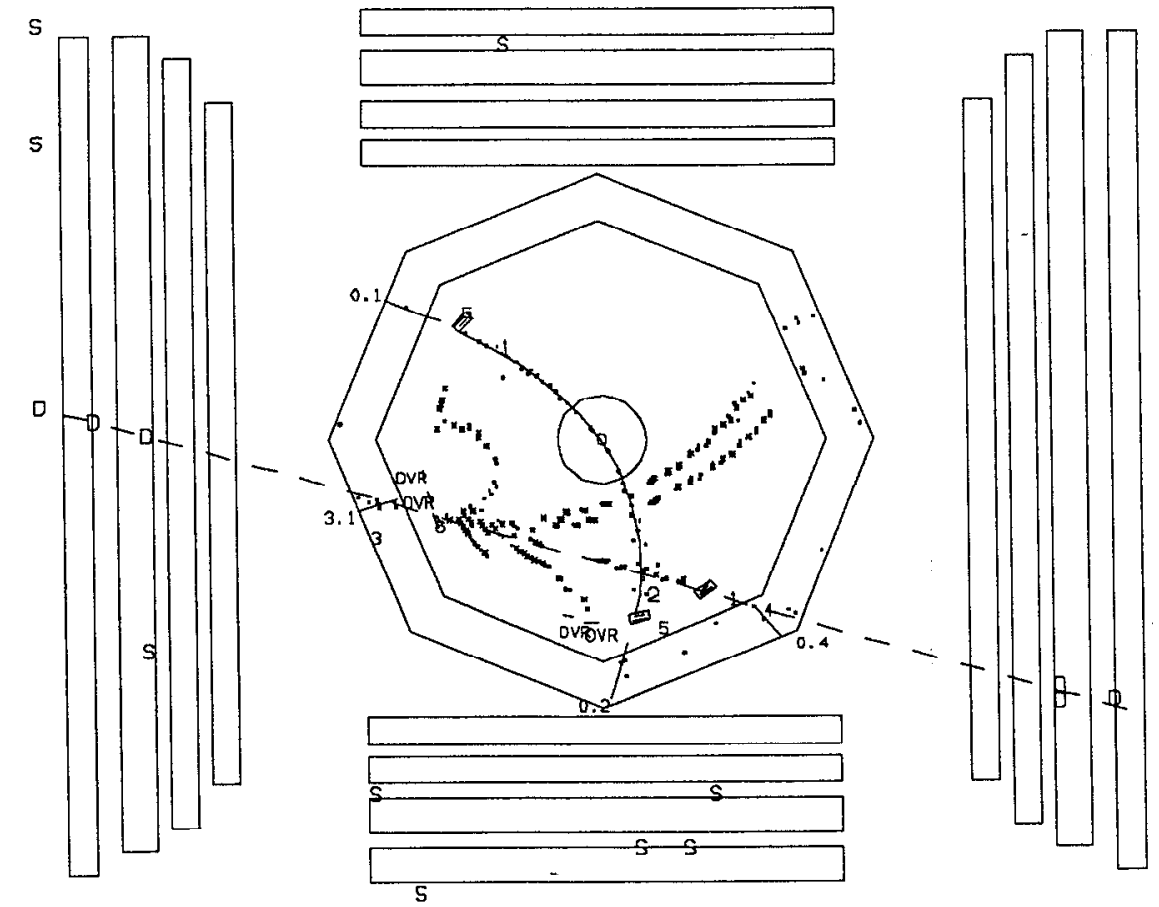

Figure 5.7. Event plot of coincidence between cosmic ray shower and two-photon production of a charged pair (probably electron positron pair). The dashed line indicates a track found by the cosminic muon rejection algorithm.

In principle, such events can simply be discarded upon inspection, but there is a 
danger in this approach. Most of the cosmic coincidences are not so clear-cut, and the criteria that one uses are inevitably somewhat subjective. This leads to the problem of calculating efficiencies for both signal events and conventional backgrounds. Some small fraction of either type of event may appear to have the same characteristics expected from the cosmic ray coincidences and thus be rejected. In principle, one can measure the loss by inspecting Monte Carlo simulated events and simply counting the number that look like the cosmic events. There are two problems with this approach, however. The first is that the Monte Carlo may not simulate the detector adequately. For example, there may in reality be occasional coherent noise in the muon layer electronics that would cause spurious signals to appear in a straight line, thus faking the passage of a muon that points in the direction of the tagging photon. Another problem, less serious but more difficult to assess, is bias in the inspection procedure. When examining Monte Carlo events that one knows not to be a cosmic coincidence, one may find reaons to reject the cosmic hypothesis more readily than one would for true data events.

To avoid these drawbacks of event inspection, algorithms have been devised to reject cosmic coincidences based on objective criteria that can be quantified. The quantification, in the form of computer algorithms, has the considerable advantage of allowing a direct and objective measurement of inefficiency for signal and conventional backgrounds from the data itself, instead of relying solely upon Monte Carlo simulation. Three distinct algorithms are applied. The first is based on the characteristics of the tagging photon's shower. The second and third look for evidence of the cosmic muon's passage through other parts of the detector, one by examining signals from the muon system layers, the other by examining drift chamber signals.

The first algorithm takes advantage of the fact that, in general, the electromagnetic shower induced by the knock-on electron is not consistent with the shower induced by a photon originating from the beam collision point. Typically, electromagnetic showers whether originating from photon conversions or bremsstrahlung of 
electrons, tend to have a fairly well defined shower axis about which the shower is approximately cylindrically symmetric. ${ }^{58}$ In addition, the length of the shower along the original particle's motion is greater than the shower's transverse size. This property is exploited in the standard reconstruction program for the liquid argon calorimeter. When combining energies from neighboring strips in a layer, the program limits the number of strips assigned to a single electron or photon shower, allowing an easier separation of two nearby photons, such as occur in the decay of an energetic $\pi^{0}$. This is a safe procedure, because nearly all photons of interest originate from near the origin, which means their shower axes are perpindicular to the layer of strips.

For a shower originating from a cosmic ray, however, the shower axis direction will generally have a component parallel to the layer; therefore, there may be more strips than expected in a given layer. In such a situation, the standard reconstruction program includes only the most energetic strips in a given layer. The rest are ignored unless there are enough different layers to make a second photon candidate. (If a second nearby photon is found, and it has an energy greater than $150 \mathrm{MeV}$, the event is discarded, as described earlier.) The first cosmic rejection algorithm looks for the extra, unassociated strips in the vicinity of the tagging photon. Specifically, the program considers the energy of all unassigned strips within $30^{\circ}$ of the tagging photon in three projections along the directions of the F, T, and U layers. The total energy is computed and a subtraction made for random noise $(1.1 \mathrm{MeV}$ per channel). The distribution in the remainder, that is, the excess unassociated energy, is shown in fig. 5.8 for a sample of radiative Bhabha events. From this figure, a cut at $+20 \mathrm{MeV}$ appears reasonable. The event discussed earlier (see fig. 5.7) yields an excess unassociated energy of $32 \mathrm{MeV}$. Note that there is a scaling factor of about 10 between raw deposited energy as measured by the strips and the actual total energy of an electromagnetic shower with shower axis perpindicular to the calorimeter layers. Therefore, a $20 \mathrm{MeV}$ excess would correspond approximately to $200 \mathrm{MeV}$ true energy, if the shower were due to a particle originating from the origin. From the radiative 
Bhabha sample, a cut at $20 \mathrm{MeV}$ yields an efficiency for preserving signal events estimated at $98.7 \pm 0.3 \%$.

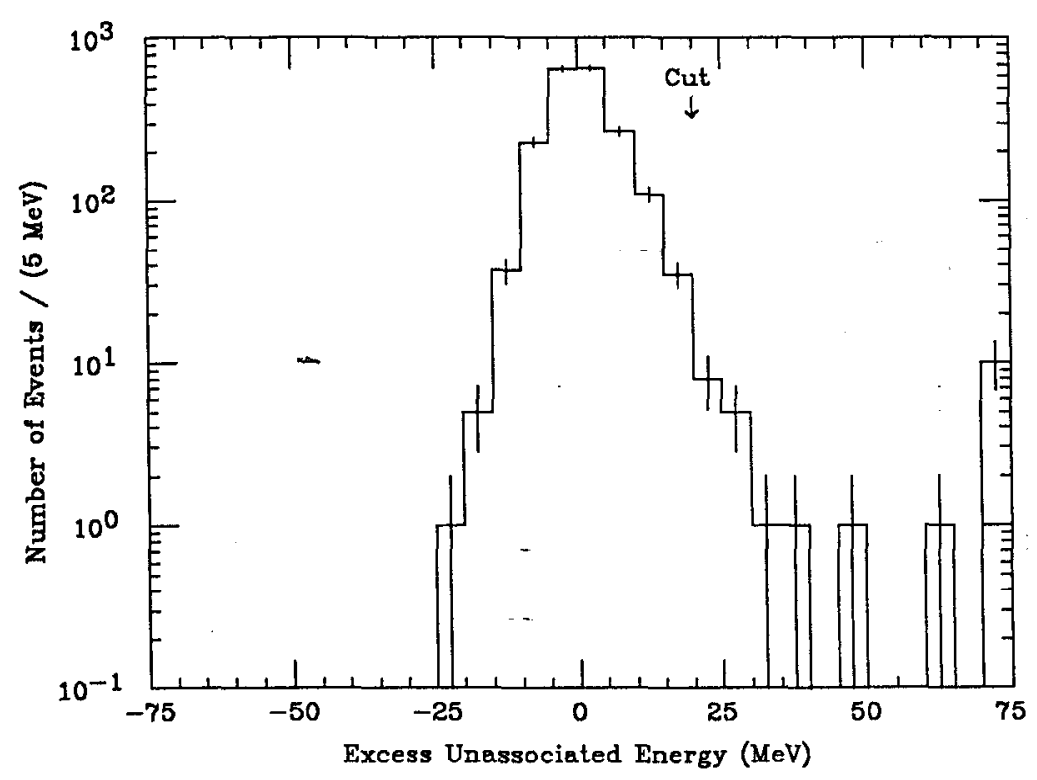

Figure 5.8. Distribution in excess unassociated energy deposition in the liquid argon calorimeter in the vicinity of photons from radiative Bhabha events. The last bin shown includes overflow events.

The second cosmic rejection algorithm is more straightforward, in that it simply looks for an alignment of muon layer hits with the tagging photon. In each of the four muon system walls, the wires in the three outer proportional tube layers lie parallel to the beam direction, allowing a direct measurement of the $\mathrm{x}$ - and $\mathrm{y}$-coordinates of a muon track. The wires in the inner layer are perpindicular to the beam direction, allowing a measure of the $\mathrm{z}$ - and either the $\mathrm{x}$ - or $\mathrm{y}$-coordinate of the muon track. The algorithm considers all muon chamber signals that have not already been assigned to charged tracks originating from the origin. All combinations in which there is a hit in each of the three outer layers of a given muon wall are examined. If three hits in three different layers are found to be aligned in the $x-y$ plane within errors(taken to be half the distance between wires in a layer), if the extrapolation of that line passes within 2 strips of the tagging photon in any calorimeter layer, and if there is a signal somewhere in the inner layer of the original muon wall, the event is rejected. The 
dashed line in fig. 5.7 is a fit found by the muon rejection algorithm.

Figure 5.9 shows another event rejected by the muon algorithm. This time, the muon enters from above, induces an electromagnetic shower in the upper right calorimeter, crosses the central drift chamber, before exiting through the the lower left calorimeter module, where it deposits a small amount of energy through ionization. The fact that the extrapolated line derived from the muon layer signals does not quite match the drift chamber signals visible in the plot suggests that the cosmic muon was deflected slightly in its collision with the knock-on electron in the calorimeter.

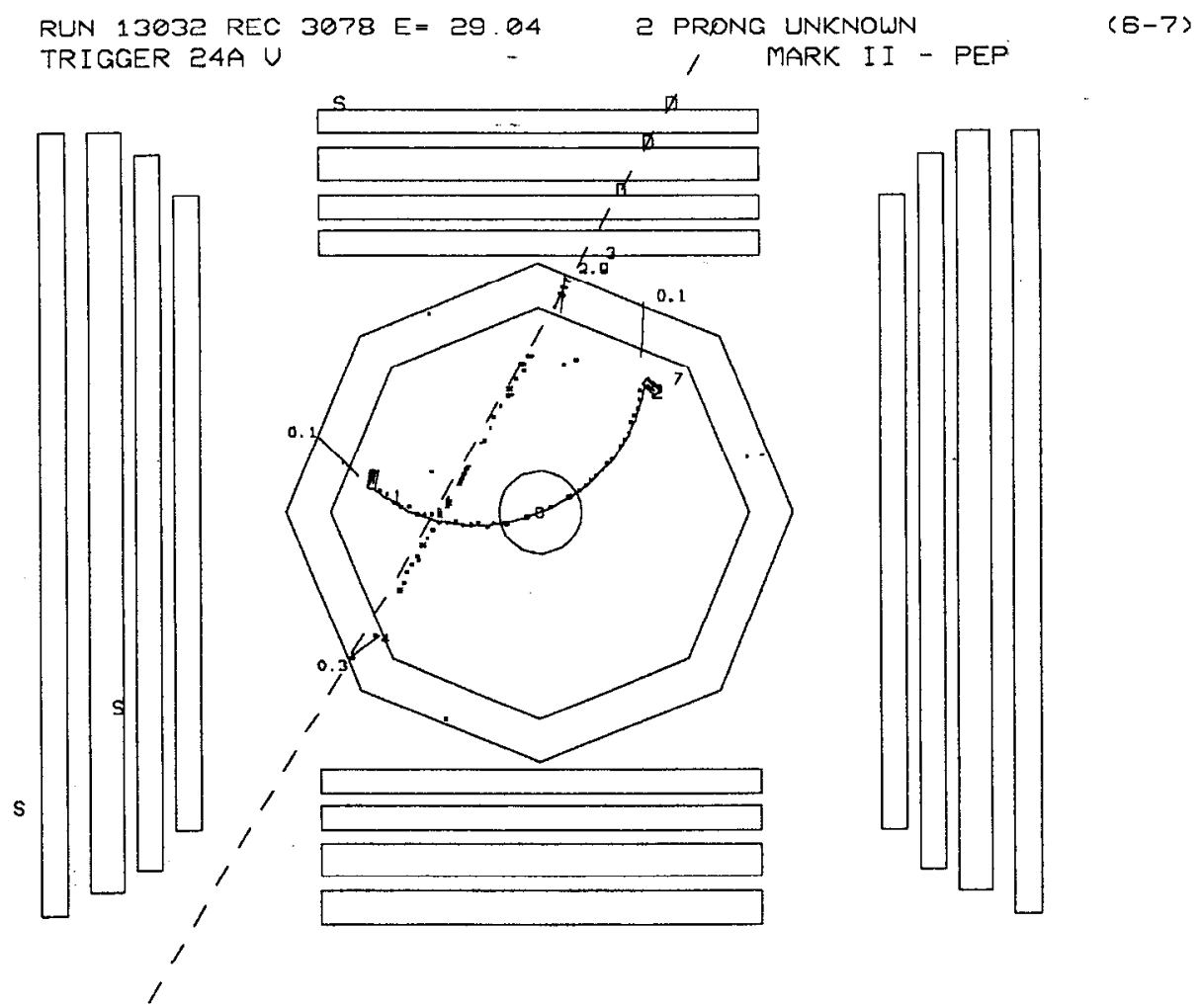

Figure 5.9. Event plot of another coincidence between cosmic ray shower and two-photon process. This event is rejected by the muon finding algorithm.

Note that in both this event and the one in fig. 5.7, the two charged tracks originating from the beam collision are nearly coplanar, i.e., back-to-back in the $x-y$ plane, as they leave the beam region. Their transverse momenta very nearly cancel 
each other. The chances are high that both events are from the two-photon process $\mathrm{e}^{+} \mathrm{e}^{-} \rightarrow \mathrm{e}^{+} \mathrm{e}^{-} \mathrm{e}^{+} \mathrm{e}^{-}$, although it is difficult to verify this because the low energies of the tracks preclude reliable identification from calorimeter energy. This high coplanarity is the motivation for the $20 \mathrm{mrad}$ cut described in an earlier section for eliminating events with false photon tags. To measure the inefficiency for signal introduced by the muon rejection algorithm, radiative Bhabha and muon pair events have been examined, along with hadronic events. The fraction of true beam-produced events rejected by the algorithm is estimated to be about $0.1 \%$.

The third cosmic rejection algorithm searches for unassigned drift chamber signals aligned with the tagging photon. Nearby clusters of drift chamber signals are identified and, if possible, extended, while requiring consistency with a straight line in the $x-y$ plane. Relatively large errors are assigned to each wire position since no correction is made for the tilt of the stereo layer wires, and since drift distances are ignored. In addition, signals from the vertex chamber are ignored because of significant coherent noise in those layers. A cosmic track candidate must have as many drift chamber signals as there are drift chamber layers in the track's path. This does not require $100 \%$ efficiency per layer, as it might appear, because most of the tracks pass through at least one layer with a direction somewhat parallel to that layer of wires, inducing more than one signal in that layer. In the case of a track intersecting a layer tangentially, more than 10 wires may record a signal. Unfortunately, this is also a property of coherent electronic noise due to crosstalk. Therefore, an additional requirement is that the track have at least one signal in at least half of the eligible drift chamber layers.

As in the muon rejection algorithm, if such a cosmic track candidate passes within two strips of the tagging photon in any calorimeter layer, the event is rejected. The event in fig. 5.9 that was rejected by the muon algorithm is also rejected by the drift chamber signal algorithm. Figure 5.10 shows the same event, where this time the dashed line indicates the fit found by drift chamber signal algorithm. Again, there 
is evidence that the muon was deflected slightly during its passage through the top calorimeter module, since the extrapolated drift chamber track slightly misses the muon chamber signals. The inefficiency of the algorithm has been estimated from the data with the same samples of radiative Bhabha, radiative muon pair and hadronic events used abovc. From these events, a conservative measure of the inefficiency (dominated by failing hadronic events) is $\theta .7 \pm 0.3 \%$. Coherent noise in the outer layers of the drift chamber and "splashback" particles from the interaction of beamproduced particles in the TOF or magnetic coil account for most of the loss.

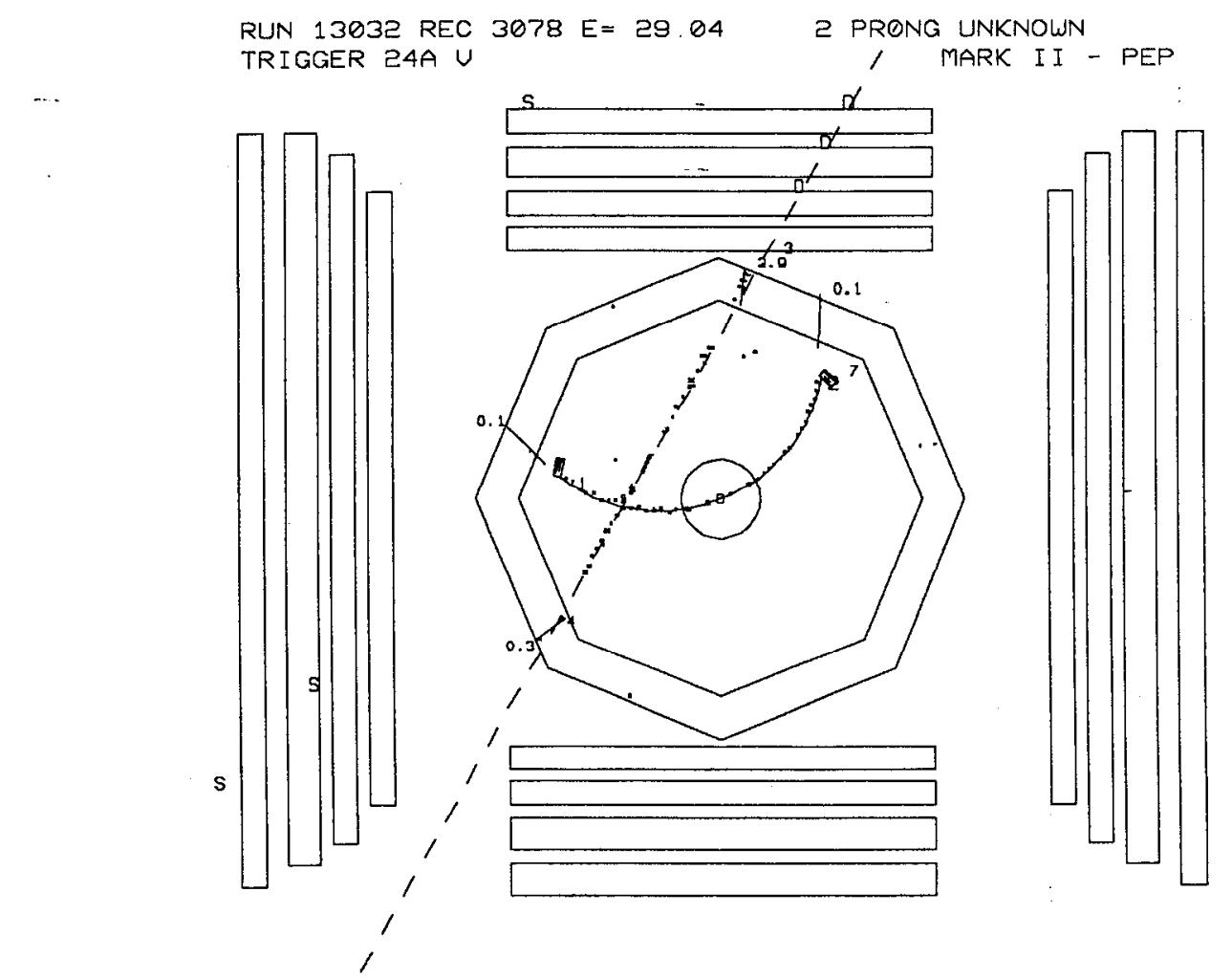

Figure 5.10. Plot of same event shown in fig. 5.9, except that the dashed line now indicates the track found from fitting the drift chamber signals.

One point should be addressed concerning the objectivity of these algorithms. Some subjectivity cannot be avoided in devising a rejection scheme. At some point, one must choose particular cuts that preserve what one considers to be true beamproduced events and reject those from cosmic ray coincidences. To avoid direct bias, 
one chooses a different sample of events from the signal events, such as radiative Bhabhas, with which to refine the algorithm and measure inefficiency. Nevertheless, one relies ultimately upon inspection ("hand-scanning"). It is inaccurate to say that subjectivity has been eliminated; one has merely found a way to measure with reasonable accuracy the effect of that subjectivity.

\subsection{Background Estimation}

Because the selection cuts described at the beginning of this chapter tend to find events with large missing energy and missing transverse momentum, not many conventional processes contribute background events. The polar angle and energy requirements on the tagging photon suppress enormously most two-photon processes, the isolation criteria suppress final state radiation and $\pi^{0}$ decay backgrounds, and the event momentum requirements suppress events with undetected particles emitted at forward angles. The remaining two-photon backgrounds are almost completcly eliminated by the requirement of missing transverse momentum.

Therefore, the remaining backgrounds are primarily those in which a particle passes through the central detector undetected. Because of the high tracking efficiency, this necessarily implies an escaped neutral particle. In the case of the signal events, of course, there are at least two neutrinos or anti-neutrinos in each event. Not surprisingly, the largest conventional background comes from the similar process of radiative tau production and decay, where again, there are at least two neutrinos missing. An additional background comes from tau pairs produced through twophoton production where the tagging photon is from an unresolved $\pi^{0}$ decay, mainly produced in $\tau \rightarrow \rho \nu_{\tau}$ decays. Mentioned earlier was the background from two-photon production of $\pi^{+} \pi^{0} \pi^{-} \pi^{0}$, in which most of the energy of one of the $\pi^{0}$ 's is lost to a photon that exits the detector through gaps in the calorimetry acceptance. The fourth background found to be non-negligible is from hard radiative hadronic events where much of the energy is lost to a very energetic photon emitted along the direc- 
tion of the beam and to undetected particles in the forward regions, particles which roughly balance the longitudinal momentum of the initial state photon. In such a case, where only two charged particles are detected in the drift chamber and a photon from $\pi^{0}$ decay forms the event tag, an undetected neutron or anti-neutron can provide enough missing transverse momentum to satisfy selection criteria.

\subsubsection{Radiative $\tau$ background}

The background from radiative tau pairs is estimated with the same Monte Carlo program(LULEPT) used for simulating signal events. Details concerning production and decay simulation can be found in Appendix A. Briefly, the program simulates most significant tau decays with approximately the correct branching ratios, although because of the one-prong discrepancy in exclusive and inclusive branching ratios, an additional correction must be applied event-by-event to both signal and radiative tau simulations. These corrections are given in the appendix. In addition, because of the well-known one-prong discrepancy, ${ }^{59}$ a systematic error of $7 \%$ is assumed for predictions from radiative tau backgounds. The background from non-radiative annihilation production of tau pairs, where the tagging photon originates from a $\pi^{0}$ decay associated with $\tau \rightarrow \rho \nu_{\tau}$ is found to be negligible, because of the isolation requirements on the photon.

\subsubsection{Two-photon $\tau$ pair production}

The background from two-photon production of tau pairs has been simulated with two different Monte Carlo programs. One, called GGDEPA, ${ }^{60}$ is based on the double equivalent photon approximation ${ }^{61}$ and has been used in analyses of various two-photon processes with the Mark II detector. It simulates the diagram in fig. $1.9 \mathrm{a}$ (with $\tau$ substituted for $\mu$ ) in the approximation that the two photons have invariant mass-squares of zero and that the cross section for producing the tau pairs can be factorized into a "luminosity function", which describes the production of the virtual photons, times the cross section for producing tau pairs through annihilation of two 
real photons. This approximation neglects quantum mechanical effects arising from interference, but it allows rapid, efficient computer simulation. The error in this approximation varies from about $10 \%$ to $40 \%,{ }^{62}$ depending on selection criteria. A conservative assumption used in this analysis is $30 \%$. A relatively small sample of events simulated with the slower Monte Carlo gives a background estimate consistent with the GGDEPA prediction, although the statistics are quite limited.

\subsubsection{Two-photon production of four pions}

The background from two-photon-produced four-pion events is also simulated with the GGDEPA program. Two-photon production of hadronic states is poorly understood. The validity of the double equivalent photon approximation is difficult to assess in a region where the production of hadrons through parton-like quarks is questionable. There is reason to believe that the Vector Dominance Model, in which the virtual photons mix quantum mechanically with neutral vector mesons(primarily the $\rho^{0}$ ), is more appropriate in describing two-photon production of hadrons at low momentum transfers. ${ }^{63}$ Therefore, the systematic error on the estimate of this background is assumed to be quite large.

To normalize the background estimate from Monte Carlo simulation, experimental measurements by the JADE and ARGUS collaborations ${ }^{64,65}$ have been used, along with the Low approximation. ${ }^{66}$ This approximation assumes

$$
\sigma\left(\mathrm{e}^{+} \mathrm{c}^{-} \rightarrow \mathrm{e}^{+} \mathrm{e}^{-} \mathrm{X}\right) \approx 2\left(\frac{\alpha}{\pi}\right)^{2}\left[\ln \left(\frac{\mathrm{E}_{\mathrm{B}}}{\mathrm{m}_{\mathrm{e}}}\right)\right]^{2} \int_{\mathrm{S}_{\text {thresh }}}^{4 \mathrm{E}_{\mathrm{B}}^{2}} \frac{d s^{\prime}}{s^{\prime}} \sigma_{\gamma \gamma \rightarrow \mathrm{X}}\left(s^{\prime}\right) f_{\mathrm{Low}}\left(\frac{\sqrt{s^{\prime}}}{2 \mathrm{E}_{\mathrm{B}}}\right)
$$

where $S_{\text {thresh }}$ is the minimum invariant mass squared for producing system $\mathrm{X}$, and the Low function is defined as

$$
f_{\text {Low }}(x)=\left(2+x^{2}\right)^{2} \ln \left(\frac{1}{x}\right)-\left(1-x^{2}\right)\left(3+x^{2}\right)
$$


From the ARGUS and JADE measurements, a functional form for the cross section for two-photon anhihilation into $\pi^{+} \pi^{0} \pi^{-} \pi^{0}$ is taken to be

$$
\sigma\left(\gamma \gamma \rightarrow \pi^{+} \pi^{0} \pi^{-} \pi^{0}\right)=70 \mathrm{nb} \frac{\left(\Gamma_{0} / 2\right)^{2}}{\left(\mathrm{M}_{\rho \rho}-\mathrm{M}_{0}\right)^{2}+\left(\Gamma_{0} / 2\right)^{2}}
$$

where the nominal mass and width are taken as

$$
\mathrm{M}_{0}=2.0 \mathrm{GeV} \quad \Gamma_{0}=0.90 \mathrm{GeV}
$$

For the purposes of this analysis, $S_{\text {thresh }}$ can be taken as $(1.5 \mathrm{GeV})^{2}$, yielding a lotal cross section $\sigma\left(\mathrm{e}^{+} \mathrm{e}^{-} \rightarrow \mathrm{e}^{+} \mathrm{e}^{-} \pi^{+} \pi^{0} \pi^{-} \pi^{0}\right)=105 \mathrm{nb}$. Because of uncertainties in the applicability of the double equivalent photon approximation in event simulation and in use of the Low approximation, a conservative systematic error of $50 \%$ is assumed for Monte Carlo rate predictions for $\mathrm{e}^{+} \mathrm{e}^{-} \rightarrow \mathrm{e}^{+} \mathrm{e}^{-} \pi^{+} \pi^{0} \pi^{-} \pi^{0}$.

\subsubsection{Hadronic event production}

Accurately predicting backgrounds from hadronic events where a neutral hadron escapes detection is quite hard. One reason is that the LUND Monte Carlo Program ${ }^{42}$ used to simulate quark-antiquark production and subsequent "hadronization" has not been verified to the level of accuracy necessary in treating events with only two detected charged tracks. Another reason is that simulation of neutral hadron interactions in the Mark II calorimeter has not been implemented with the required accuracy. Especially troubling is that neutron interaction simulations cannot be directly checked with the data. For these reasons, the estimate to be presented for hadronic background is not used in setting limits on new heavy lepton production. This is a conservative approach, since inclusion of additional backgrounds can only improve derived upper limits. 


\subsection{Efficiency Corrections for Detector Simulation}

The detector simulation of the Monte Carlo program does not always reproduce the backgrounds actually seen in the data. In some cases this is because of inadequate modelling of detector geometry; in others, the program does not account for backgrounds that are independent of the primary electron-positron interaction.

The major corrections for incorrect detector response simulation involve the timeof-flight scintillator counters, the liquid argon calorimeter, and noise in the small angle tagger. As described in chapter 4 , there is a loss in efficiency due to cracks in azimuth between the counters. The efficiency per track for Bhabha electrons of the TOF latch (necessary for charged track secondary trigger) is $98.7 \%$. The Monte Carlo predicts a value of essentially $100 \%$. Since both charged tracks in an event must induce a latched TOF signal, the total trigger efficiency is the square of the efficiency per track, yielding $97.4 \%$. The efficiencies are independent in signal events because angular correlations in azimuth between the two tracks are much broader than the extremely narrow $\left(\approx 0.25^{\circ}\right)$ gaps in acceptance. Also mentioned in chapter 4 was an additional loss in TOF efficiency for very low momentum tracks, due to inflight hadron decays and to material in front of the counters near each end. The loss from decays is simulated properly and requires no correction, but the slight loss at the counter ends leads to an unsimulated inefficiency in the range $0.2-0.4 \%$ when averaged over $|\cos \theta \leq 0.7|$ for hadrons with momentum less than $800 \mathrm{MeV}$. To account for these inaccuracies in TOF simulation, an efficiency correction of $97.0 \pm 0.5 \%$ is applied to all Monte Carlo estimates.

Monte Carlo simulation of the liquid argon calorimeter has been tested with electrons from $\mathrm{e}^{+} \mathrm{e}^{-} \rightarrow \mathrm{e}^{+} \mathrm{e}^{-} \mathrm{e}^{+} \mathrm{e}^{-}$in the data, as described in chapter 4. It was found that an algorithm that slightly smears the reconstructed Monte Carlo-produced shower improves agreement with efficiencies derived from the data. The remaining discrepancy constitutes a $0.7 \%$ efficiency loss not modelled by the Monte Carlo for photon energies in the range $0.8-2.0 \mathrm{GeV}$ with a distribution falling like $1 / \mathrm{E}_{\gamma}$. 
One failing of this efficiency study is that it tests Monte Carlo simulation of calorimeter response to electron showers, not to photon showers. It is difficult to measure directly and accurately the calorimeter response to low energy photons at PEP beam energies. One can kinematically fit planar radiative Bhabha events, using only the directions of the electrons and photon, to determine the photon energy. In principle, this allows one to directly compare reconstructed photon energy with the "true" value. In practice, however, with the integrated luminosity available to this analyis, one cannot obtain a sufficient number of low-energy photon candidates with energies determined accurately by the fit. The derived efficiency cannot be measured to better than a few percent for any narrow range in photon energy.

A photon response study ${ }^{51}$ was performed many years ago on Mark II data taken at SPEAR on the $\mathrm{J} / \psi$ resonance $(\sqrt{\mathrm{s}}=3.1 \mathrm{GeV})$, using the decay $\psi \rightarrow \pi^{+} \pi^{-} \pi^{0}$. In this technique, the momenta of the two charged pions and that of one photon from the $\pi^{0}$ decay are measured. These momenta overconstrain the second photon's direction and encrgy, allowing a kinematic fit to detcrmine their-values, thus providing a direct measurement of photon efficiency and resolution. The agreement between data and Monte Carlo was found to be quite good. Because the shower reconstruction algorithms were somewhat modified after the Mark II was moved to PEP, the study has been redone for this analysis, using a small data sample from SPEAR that was still available. Qualitatively, the results agree with the previous study, but again the accuracy of the Monte Carlo cannot be verified to better than a few percent. None of these studies indicate any measurable discrepancy in photon shower simulation between Monte Carlo and data, and comparison of Monte Carlo generated electrons and photons give efficiencies that also agree to within a few percent. As a quite conservative estimate then, it is assumed that the difference between electron and photon initiated showers gives rise to an uncertainty in photon detection efficiency of $3 \%$.

Another small inefficiency arises from noise in module 8 of the liquid argon 
calorimeter (azimuth from $315^{\circ}$ to $360^{\circ}$ ). Towards the end of PEP data-taking, the electronics gain for that module was reduced, as a test in preparation for SLC running where the previous gain values would have led to channel saturation for the highest shower energies. After the gain change and necessary associated changes to pedestals and other calibration constants, the relative noise in the module increased substantially. As a result, for about the last $50 \mathrm{pb}^{-1}$ of data, false energy depositions in module 8 occasionally mislead the shower reconstruction programs into associating the wrong $\mathrm{F}$ and $\mathrm{T}$ layers together. This not only causes the shower to be placed in the wrong location, but it also degrades the energy resolution. The resulting inefficiency has been measured with the $\mathrm{e}^{+} \mathrm{e}^{-} \rightarrow \mathrm{e}^{+} \mathrm{e}^{-} \mathrm{e}^{+} \mathrm{e}^{-}$electron sample and found to be about $0.4 \%$. In summary, the efficiency correction for inaccuracy of calorimeter simulation. is taken to be $98.9 \pm 3.0 \%$.

Background rejection based on energy deposited in the SAT calorimeter was discussed earlier in this chapter. The inefficiency due to these cuts is estinated to be $4.6 \pm 0.6 \%$, a loss not simulated by the standard Monte Carlo program.

Each of the algorithms that reject cosmic ray coincidences incurs a small ineffciency. To be conservative, the correlations among the inefficiencies are ignored and the three efficiency corrections simply multiplied together. The result is a correction factor of $97.9 \pm 0.5 \%$.

All of the efficiency corrections for inadequate detector simulation are shown in Table 5.1. The errors are added in quadrature and the efficiency factors multiplied together to give $89 \pm 3 \%$.

\subsection{Comparison of Data to Background Estimate}

When the event selection criteria described earlier in the chapter are applied to the data $\left(104 \mathrm{pb}^{-1}\right)$, including the various veto conditions, only 14 events remain. When the same criteria are applied to the first three background samples (normalized to the data's integrated luminosity) and all efficiency corrections applied, a total of 
Table 5.1. Given below are efficiency corrections due to inaccurate Monte Carlo simulation of detector response and occupancies.

\begin{tabular}{|l|c|}
\hline Time-of-Flight scintillators & $.970 \pm .005$ \\
\hline Lead-Liquid Argon Calorimeter & $.989 \pm .031$ \\
\hline Small Angle Tagger (Calorimeter) & $.954 \pm .006$ \\
\hline Cosmic Ray Coincidence Algorithms & $.979 \pm .005$ \\
\hline \multicolumn{1}{|c|}{ Total (Detector simulation) } & $.89 \pm .03$ \\
\hline
\end{tabular}

12.3 \pm 1.7 events survive, as shown in Table 5.2. The errors given are systematic; they do not include statistical fluctuations from these average expected values, which dominate the uncertainty in the number of events actually observed. No error is given for the radiative hadronic background estimate for the reasons mentioned earlier.

Table 5.2. Given are final estimates for contributions from the four main backgrounds described earlier. Values are normalized to $104 \mathrm{pb}^{-1}$ of integrated luminosity. Errors are systematic only.

\begin{tabular}{|l|c|}
\hline $\mathrm{e}^{+} \mathrm{e}^{-} \rightarrow \tau^{+} \tau^{-} \gamma$ & $9.7 \pm 1.4$ \\
\hline $\mathrm{e}^{+} \mathrm{e}^{-} \rightarrow \mathrm{e}^{+} \mathrm{e}^{-} \tau^{+} \tau^{-}$ & $2.3 \pm 1.0$ \\
\hline $\mathrm{e}^{+} \mathrm{e}^{-} \rightarrow \mathrm{e}^{+} \mathrm{e}^{-} \pi^{+} \pi^{0} \pi^{-} \pi^{0}$ & $0.3 \pm 0.2$ \\
\hline $\mathrm{e}^{+} \mathrm{e}^{-} \rightarrow \mathrm{e}^{+} \mathrm{e}^{-} \mathrm{q} \overline{\mathrm{q}}$ & $1.7 \pm ?$ \\
\hline \multicolumn{1}{|c|}{ Total } & $12.3 \pm 1.7$ \\
\hline
\end{tabular}

In total number, the background estimates are consistent with what is observed in the data. To assess whether this agreement can be trusted, one must examine various distributions. Figure 5.11 shows the distribution in tagging photon energy for data and background. Within the limited statistics, the distributions agree fairly well in shape and normalization. The invariant mass of the two charged particles together(assumed pions) is plotted in fig. 5.12. Again, the agreement is good. Fig- 
ure 5.13 shows the invariant mass of the photon combined with each charged track (2 entries per event). Figure 5.14 shows the distribution in total energy for the photon and two charged particles; the dominance of the photon energy in the total is seen in the resemblance between figures 5.11 and 5.14. This can also be seen directly in fig. 5.15, which shows the distribution in momenta of the charged tracks. Note that one event selection requirement is that each charged particle momentum be less than $4 \mathrm{GeV}$.

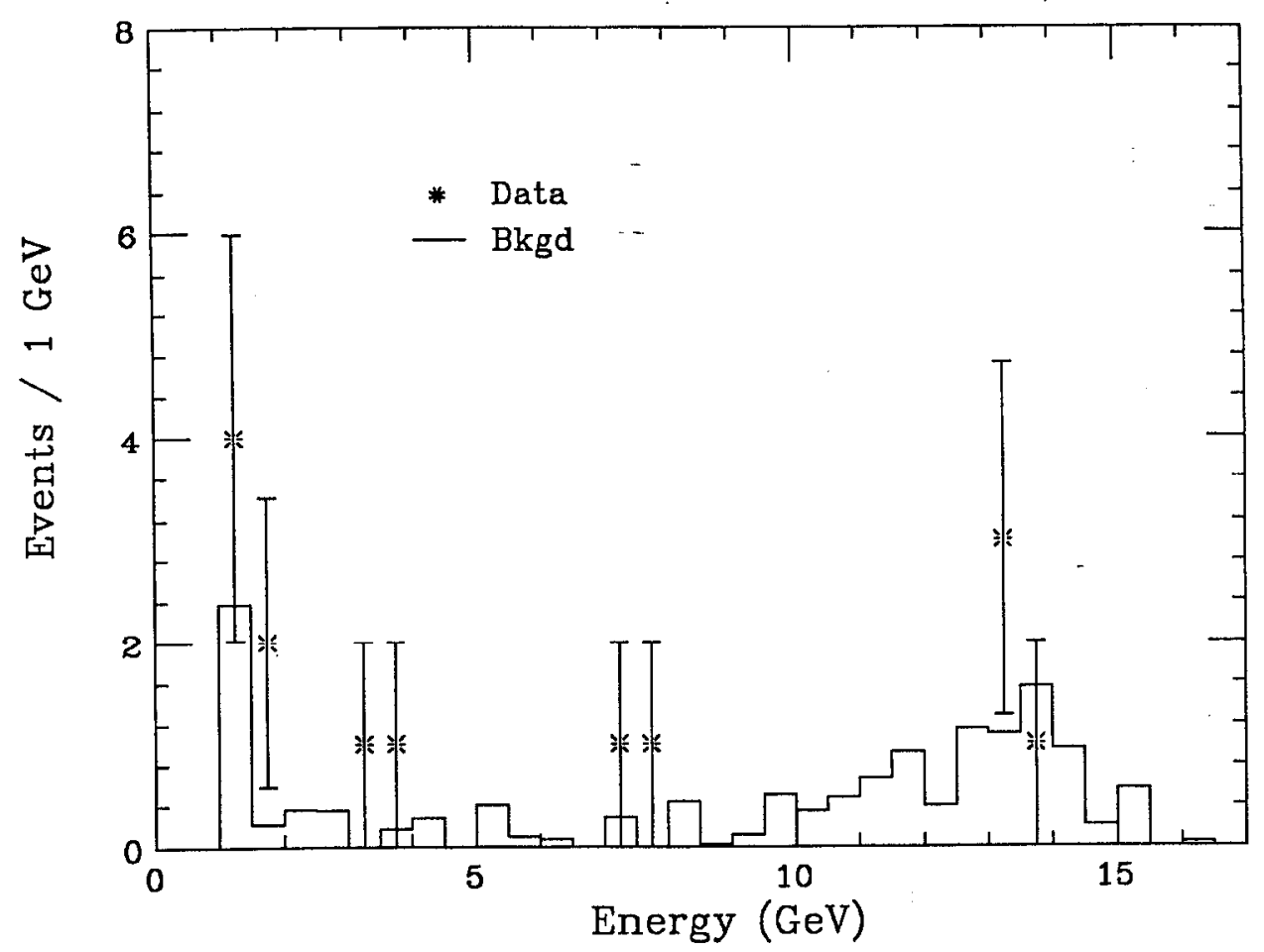

Figure 5.11. Distribution in energy of tagging photon for data and estimated backgrounds.

As discussed in the first chapter, electron identification cannot be used for event selection, given the typical charged particle momenta of surviving events. Muon identification is even less reliable. But there is enough information available to allow statistical separation of electrons from pions. For tracks with energies greater than several hundred $\mathrm{MeV}$, the calorimeter energy deposition will be higher on average for electrons than for hadrons. The energy deposition patterns (size and shape) 


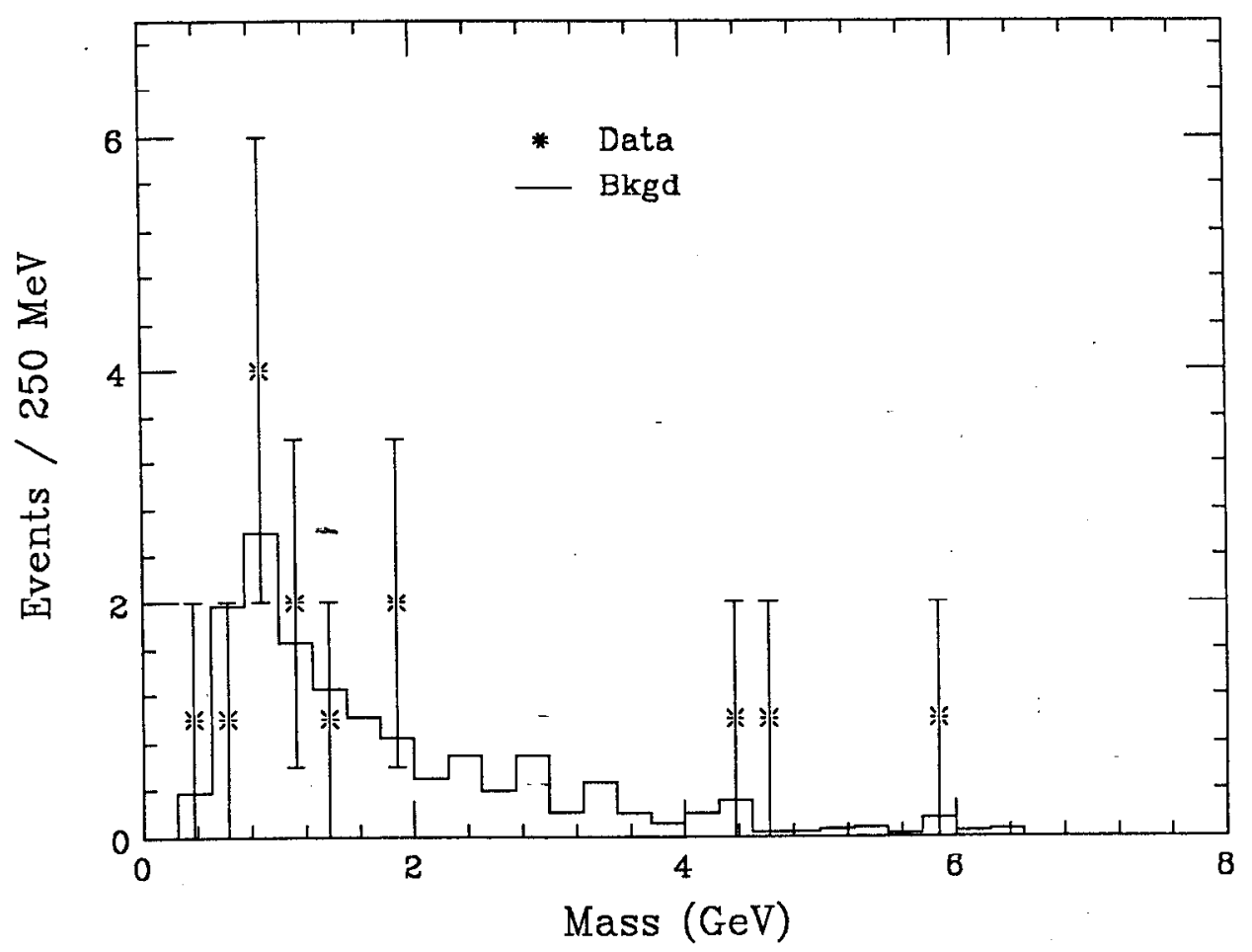

Figure 5.12. Distribution in invariant mass of the two charged particles taken together for data and estimated backgrounds.

for electrons and hadrons also differ on average. In addition, for the lowest energy tracks, times measured by the TOF scintillators can statistically distinguish electrons from hadrons. These differences have been exploited by a sorting program, known as LAESEP,${ }^{67}$ that identifies electron and pion candidates with $60-90 \%$ efficiency and with misidentification probabilities less than $15 \%$ for momenta of interest here. The algorithm has been applied to surviving events in the data and to Monte Carlo background samples. As shown in Table 5.3, the agreement in electron/pion combinations is quite good. The large number of events with one or more ambiguous tracks confirms, however, that it would be unwise to select events based upon electron and pion identification.

\subsection{Limits on a New Heavy Lepton Doublet}

From the previous section, it is clear that no signal for new heavy lepton production emerges from the data. The next question is how well one can exclude the 


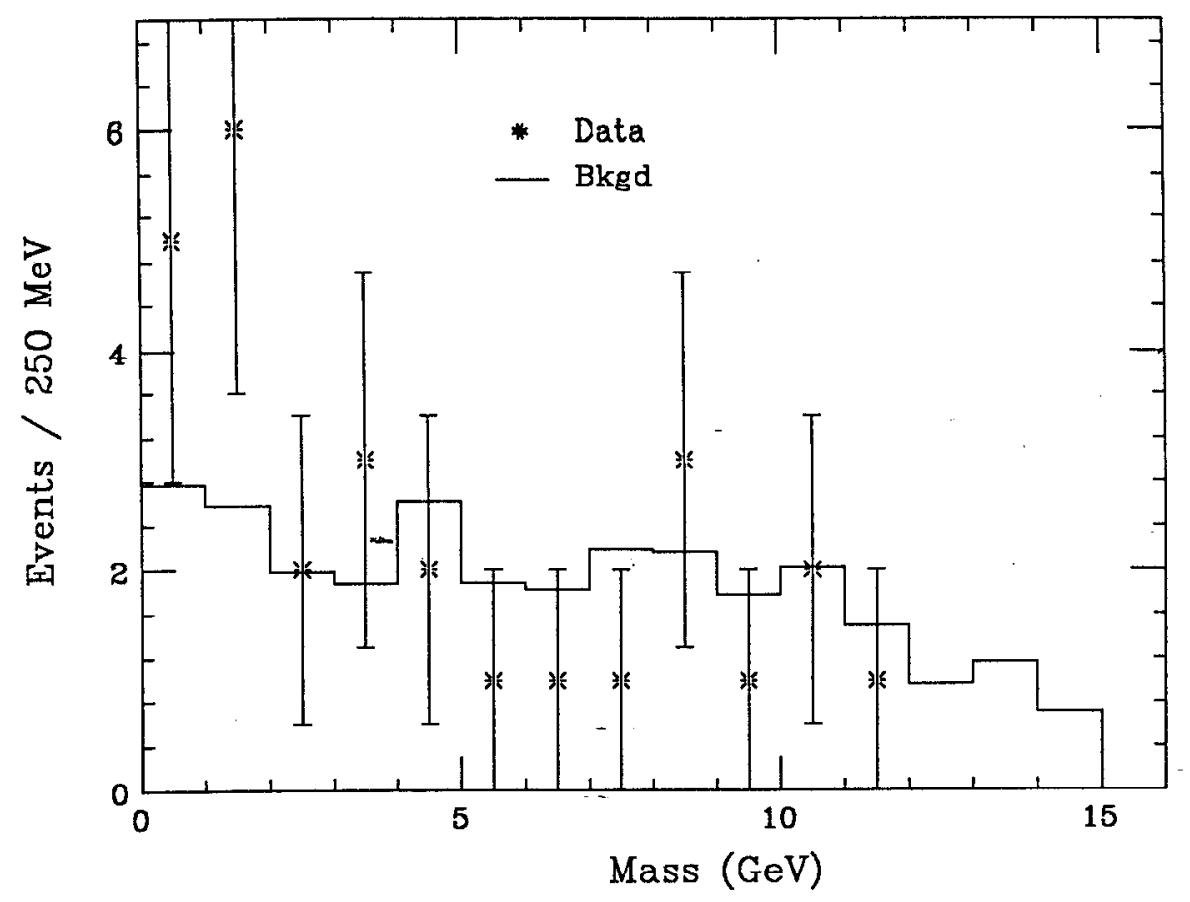

Figure 5.13. Distribution in invariant mass of charged-neutral combinations (two entries per event) for data and estimated backgrounds.

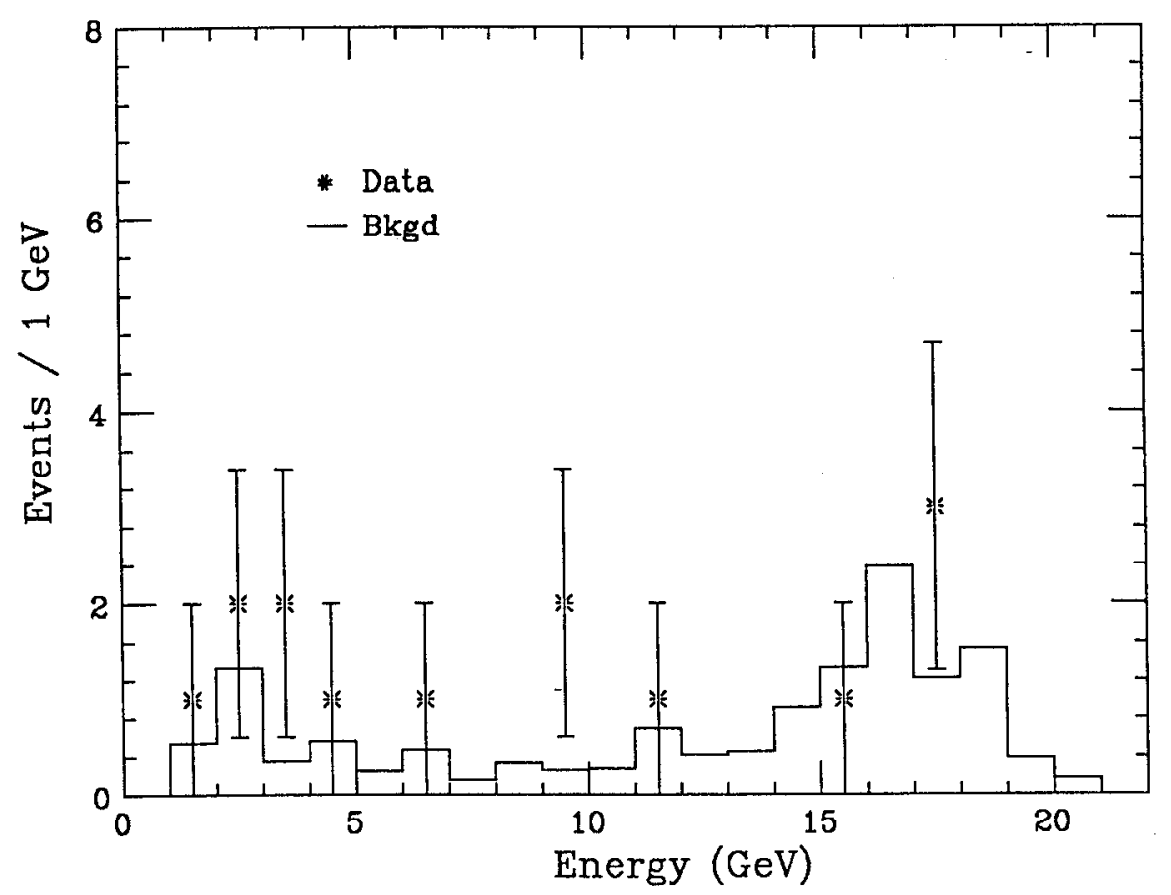

Figure 5.14. Distribution in total energy of tagging photon and two charged tracks for data and estimated backgrounds. 


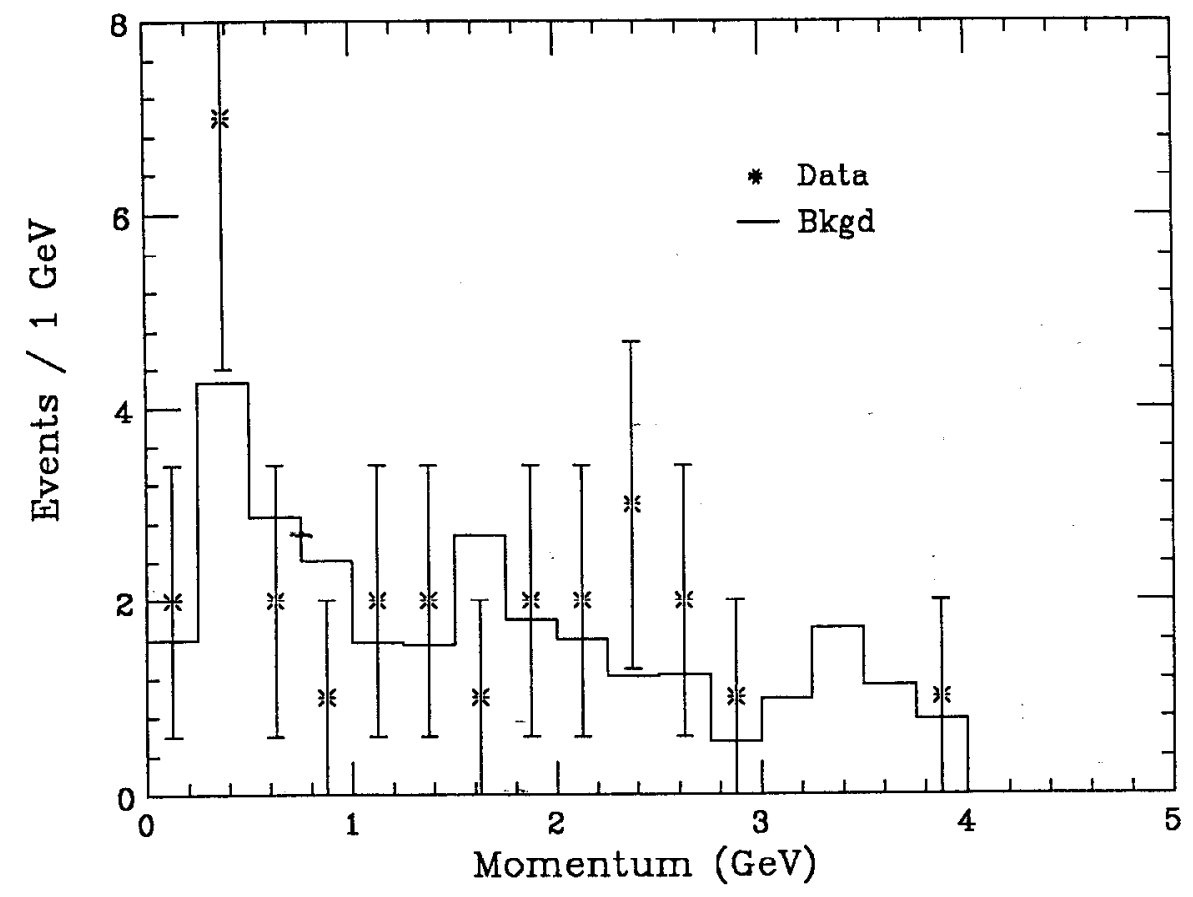

Figure 5.15. Distribution in single charged track momentum (two entries per event) for data and estimated backgrounds.

Table 5.3. Shown is the distribution in electron/pion candidates for data and (background) events surviving all cuts.

\begin{tabular}{|l|c|c|c|}
\hline & \multicolumn{3}{|c|}{ Particle Identity } \\
& Electron & Pion & Ambiguous \\
\hline Electron & $1(0.7)$ & $1(2.6)$ & $2(1.0)$ \\
\hline Pion & - & $4(3.3)$ & $4(3.1)$ \\
\hline Ambigous & - & - & $2(1.5)$ \\
\hline
\end{tabular}

existence of heavy leptons with these results. As a first step, one can add the heavy lepton distributions expected from the exemplary signal cases $\mathrm{A}-\mathrm{C}$, defined earlier, to the background distributions in figures 5.11-5.15. The results are shown in figures 5.16-5.20, From these distributions, it is clear simply from the integrated areas that all three exemplary cases can be safely excluded.

In general, one can determine the confidence level of exclusion in many different 


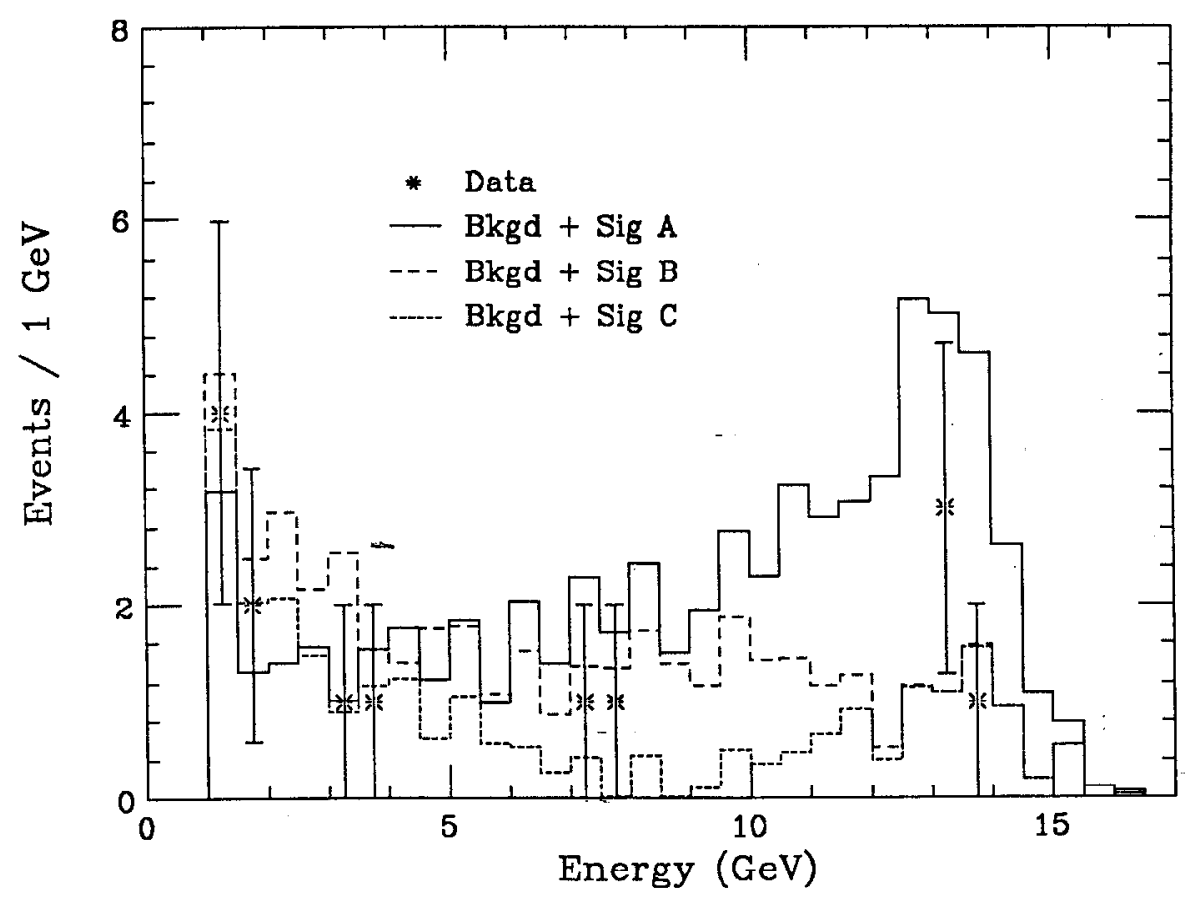

Figure 5.16. Distribution in energy of tagging photon for data and estimated backgrounds plus signals $\mathrm{A}, \mathrm{B}$, and C.

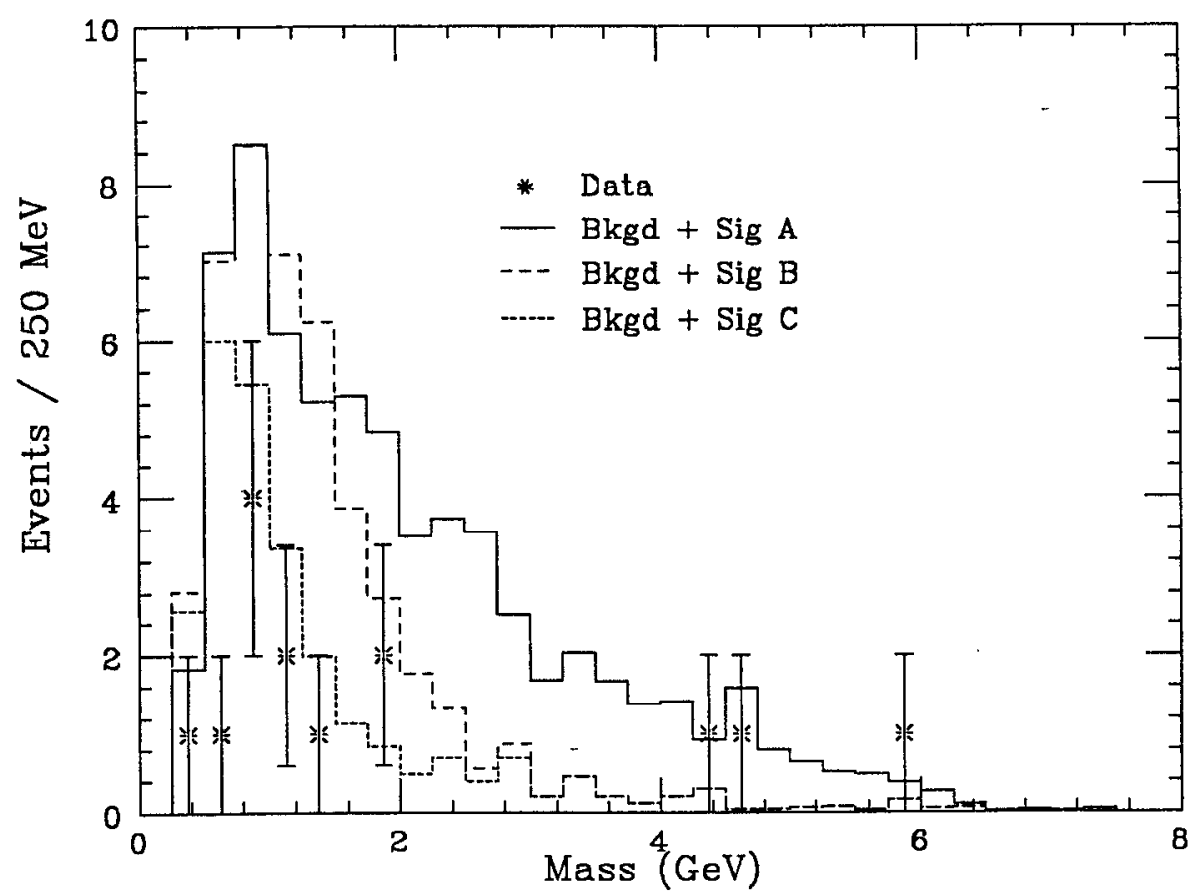

Figure 5.17. Distribution in invariant mass of the two charged particles taken together for data and estimated backgrounds plus signals $\mathrm{A}, \mathrm{B}$, and $\mathrm{C}$. 


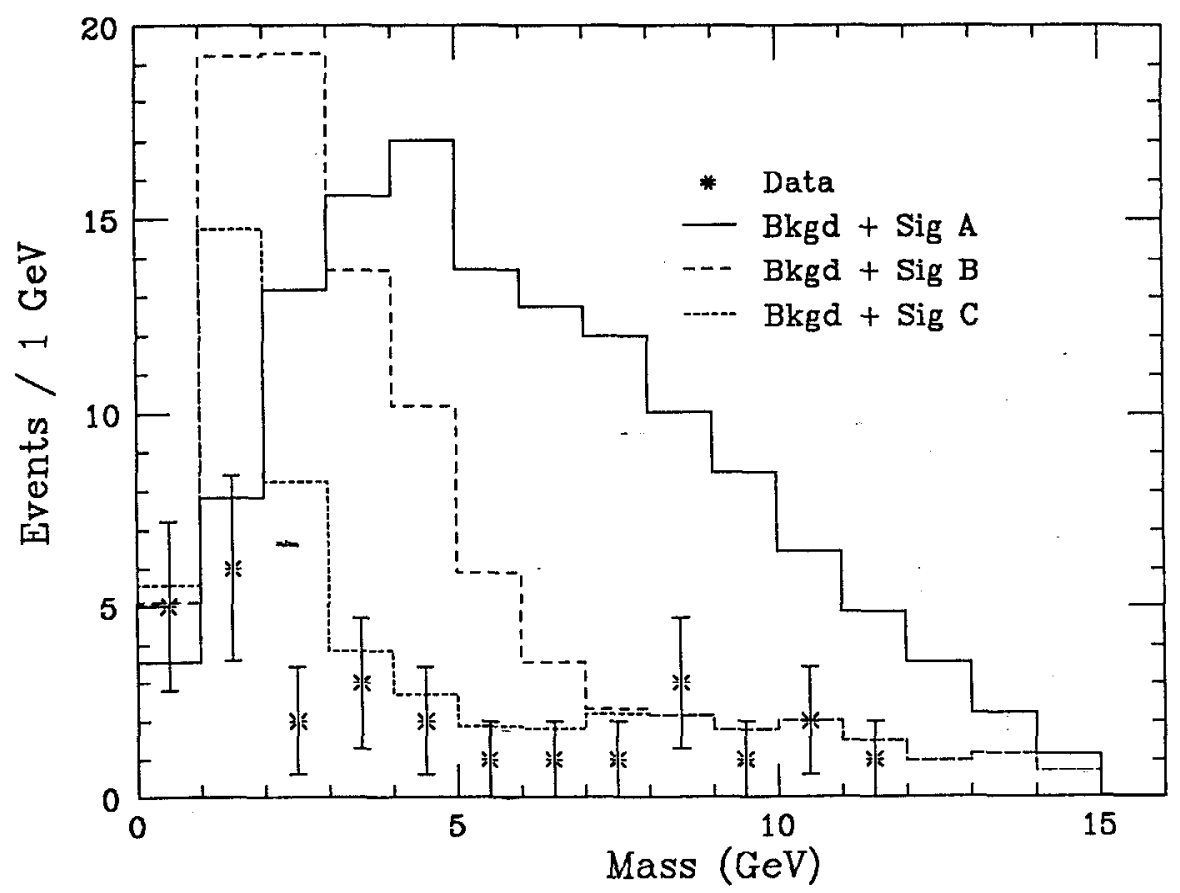

Figure 5.18. Distribution in invariant mass of charged-neutral combinations (two entries per event) for data and estimated backgrounds plus signals $\mathrm{A}, \mathrm{B}$, and $\mathrm{C}$.

ways. For example, one can compare both the shapes and total numbers of events seen in the data to that expected from the sum of backgrounds and hypothetical signal for one or more of the distributions shown in figures 5.16-5.20. The approach taken in this analysis, which is simpler and more conservative, is to compare only total numbers of events. When the total number of surviving events is as low as occurs in this analysis, it is necessary to apply techniques based on Poisson statistics. In the simplest case, the systematic errors on background and signal estimates are negligible compared to the expected statistical fluctuations on the expected value of their sum. The confidence level of excluding the signal is then taken as ${ }^{68}$

$$
\text { C.L. } \equiv 1-\frac{\sum_{n=0}^{N_{D}} \frac{\lambda_{T}^{n}}{n !} e^{-\lambda_{T}}}{\sum_{n=0}^{N_{D}} \frac{\lambda_{B}^{n}}{n !} e^{-\lambda_{B}}}
$$




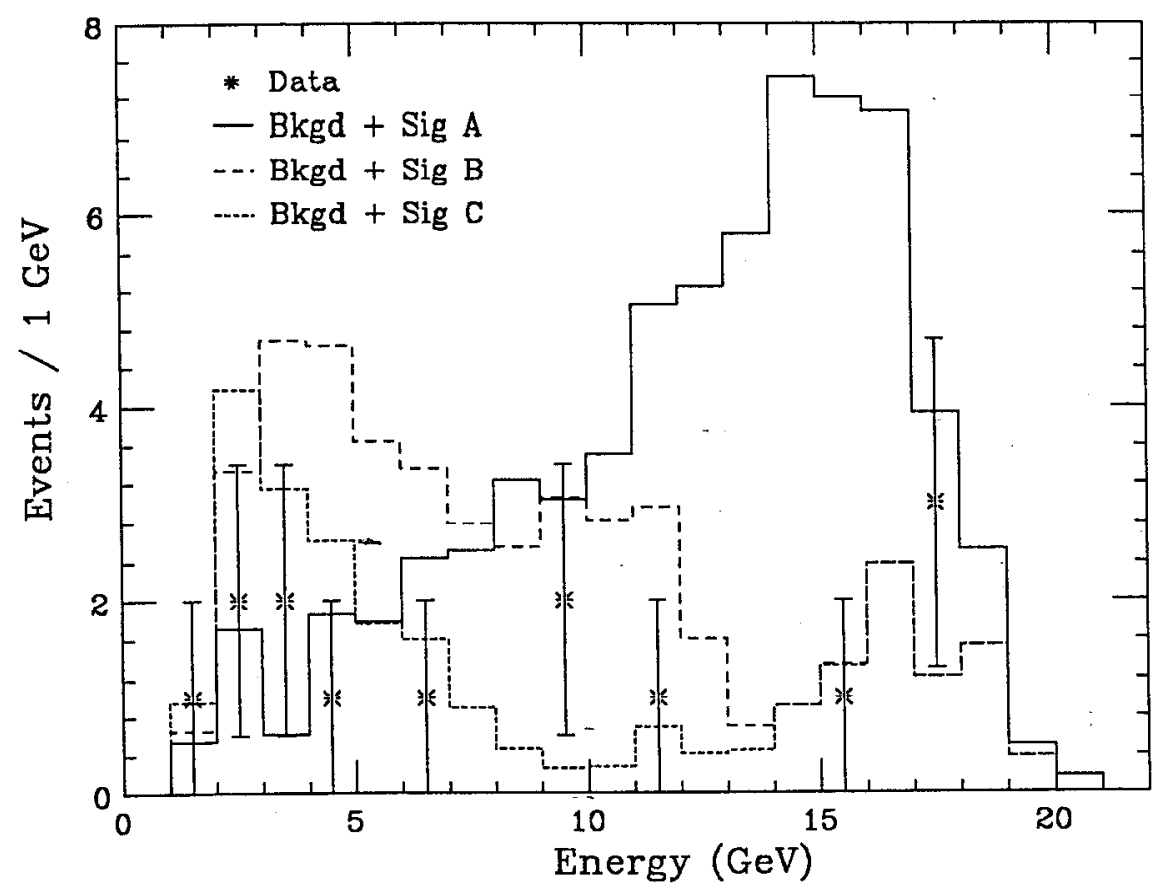

Figure 5.19. Distribution in total energy of tagging photon and two charged tracks for data and estimated backgrounds plus signals A, $\mathrm{B}$, and $\mathrm{C}$.

where

$$
\begin{aligned}
N_{\mathrm{D}} & \equiv \text { Number of events observed in data } \\
\lambda_{\mathrm{B}} & \equiv \text { Number of events expected from background } \\
\lambda_{\mathrm{S}} & \equiv \text { Number of events expected from signal } \\
\lambda_{\mathrm{T}} & \equiv \lambda_{\mathrm{B}}+\lambda_{\mathrm{S}}
\end{aligned}
$$

This definition of confidence level has the virtue that it explicitly takes into account the knowledge that the number of background events in the sample has been measured to be less than or equal to $N_{D}$. Put another way, the confidence level for an upper limit on $\lambda_{\mathrm{S}}$ is one minus the probability that the average expected background $\lambda_{\mathrm{B}}$ plus $\lambda_{S}$ would give rise to a fluctuation down to 14 or less, given that we already know that the background has fluctuated that low. This distinction is most important when an estimated background is much greater than the number of events observed.

In this analysis, statistical fluctuations away from the average expected sum of 


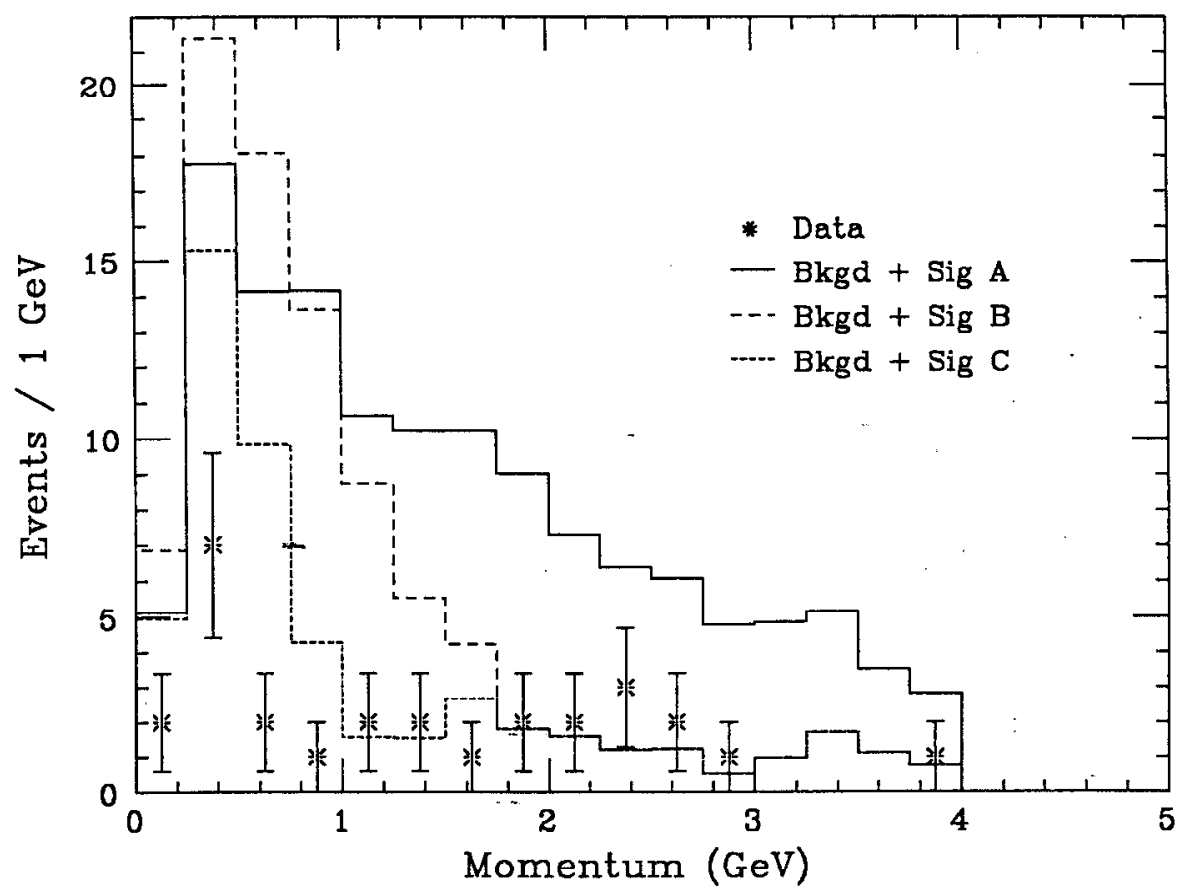

Figure 5.20. Distribution in single charged track momentum (two entries per event) for data and estimated backgrounds plus signals $\mathrm{A}, \mathrm{B}$, and $\mathrm{C}$.

background plus signal do dominate the error, but systematic errors on those expected values cannot be completely neglected. The approach taken here for treating the systematic error is to assume that if the calculated expected value of a rate is $\lambda \pm \sigma_{\lambda}$, then the differential probability that the true value $\lambda_{\text {true }}$ would lead to the calculated value is given by a Gaussian distribution:

$$
\mathrm{P}_{\text {Gauss }}\left(\lambda_{\text {true }} \rightarrow \lambda\right)=\frac{1}{\sqrt{2 \pi} \sigma_{\lambda}} \exp \frac{-\left(\lambda-\lambda_{\text {true }}\right)^{2}}{2 \sigma_{\lambda}^{2}}
$$

Strictly speaking, the value of $\sigma_{\lambda}$ should depend on the value of $\lambda_{\text {true }}$, but for small $\sigma_{\lambda}$, the distinction is unimportant. The differential probability that the true value $\lambda_{\text {true }}$ would simultaneously lead to a Monte Carlo prediction of $\lambda$ and an observed number of 14 or less in the data is then taken to be

$$
\left.\mathrm{P}_{\text {joint }}\left(\lambda_{\text {true }} ; \lambda, \mathrm{N}_{\mathrm{D}} \leq 14\right]\right)=\mathrm{P}_{\text {Gauss }}\left(\lambda_{\text {true }} \rightarrow \lambda\right) \times \mathrm{P}_{\text {Poisson }}\left(\lambda_{\text {true }} \rightarrow\left[\mathrm{N}_{\mathrm{D}} \leq 14\right]\right)
$$


If one assumes that, aside from the Monte Carlo prediction, there is no a priori preference for any value of $\lambda_{\text {true }}$, then the total probability that one would estimate a value $\lambda$ and observe $N_{D}$ or fewer events is the integral of this expression over all physical values of $\lambda_{\text {true }}$ :

$$
\mathrm{P}\left(\lambda, \mathrm{N}_{\mathrm{D}}\right)=\frac{1}{\sqrt{2 \pi} \sigma_{\lambda}} \int_{0}^{\infty} d \lambda^{\prime} \exp \left\{-\frac{\left(\lambda^{\prime}-\lambda\right)^{2}}{2 \sigma_{\lambda}^{2}}-\lambda^{\prime}\right\} \sum_{i=0}^{\mathrm{N}_{\mathrm{D}}} \frac{\left(\lambda^{\prime}\right)^{i}}{i !}
$$

For illustration, the hypothesis that the background $\left(\lambda=12.3 ; \sigma_{\lambda}=1.7\right)$ would produce 14 or fewer events in the data yields the probability $73 \%$, indicating reasonable consistency between data and background, as one would expect if there were no heavy lepton signal. If this probability were very close to unity, one would suspect the presence of unexplained backgrounds or of a signal. If the number were very small, on the other hand, one would suspect the background had been overestimated. If an experiment is repeated many times, one expects an average probability near $50 \%$.

The confidence level with which one can exclude a given signal hypothesis is $1-\mathrm{P}\left(\lambda_{\mathrm{T}}\right) / \mathrm{P}\left(\lambda_{\mathrm{B}}\right)$, where $\mathrm{P}$ is calculated from eqn. 5.1. Using this technique, one finds for the exemplary cases A, B, and C, confidence levels for exclusion of $>99.9 \%$, $>99.9 \%$, and $95.2 \%$, respectively.

In order to confidently exclude a region in the $M_{L}-M_{\nu_{L}}$ plane, as in fig. 1.6, it is necessary to generate many Monte Carlo samples at different points in the plane. A confidence level for excluding each point can be calculated, allowing an interpolation between points to find contours of exclusion at a particular confidence level, such as $95 \%$. In generating samples, one must take care to assure closely spaced points where the confidence level varies rapidly, in order to minimize error due to the interpolation. Figure 5.21 shows the 86 points generated for this analysis. The high density of points along the bottom and left reflect rapid variations in confidence levels calculated in this region.

Tables 5.4, 5.5, 5.6, and 5.7, shows the numbers of events predicted from the 


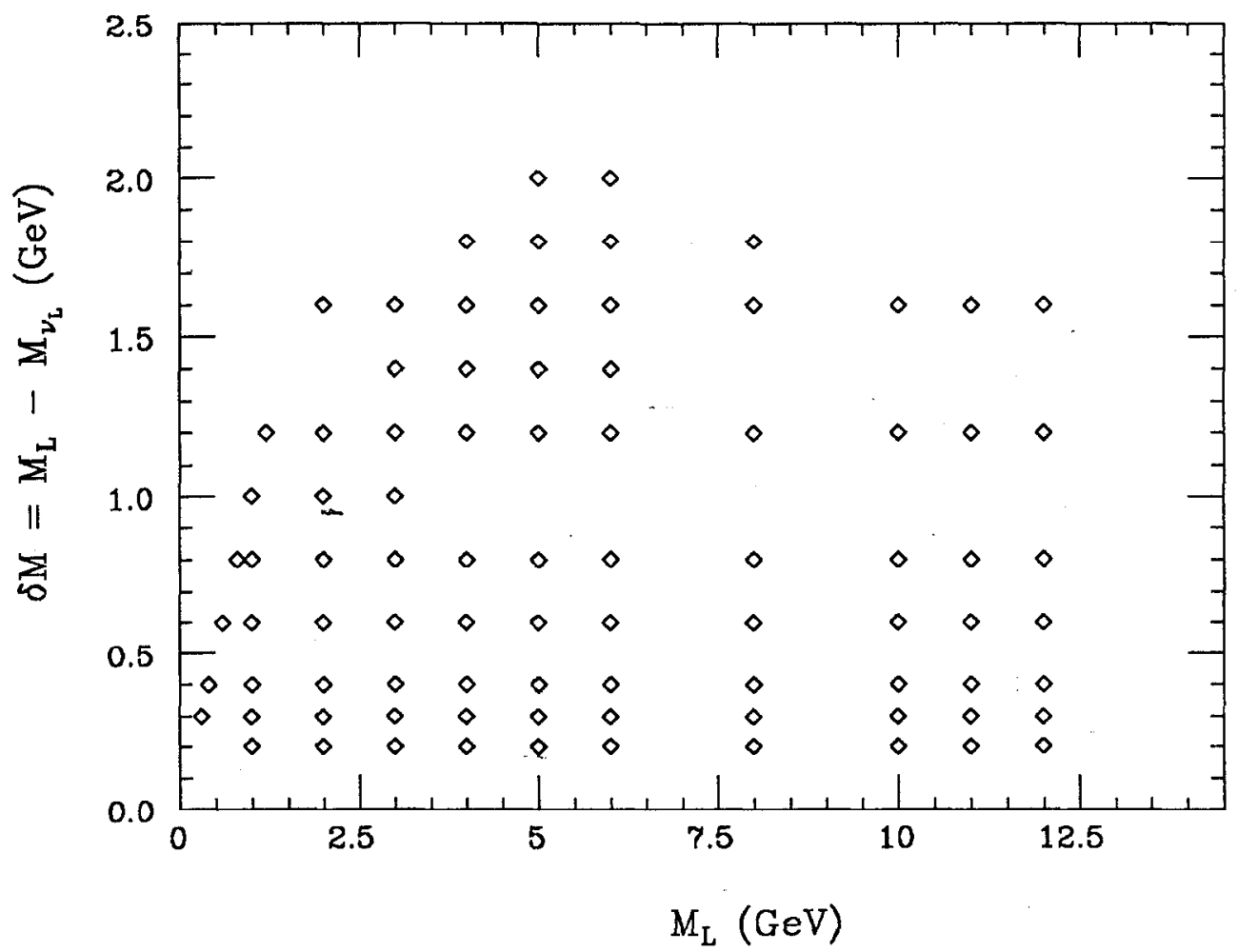

Figure 5.21. Distribution of Monte Carlo event samples in plane of mass difference vs charged mass.

Monte Carlo simulation (including all efficiency corrections) with the estimated errors. The last column gives the confidence level with which the lepton doublet can be excluded. From these numbers, one can interpolate between points to get confidence level contours. Empirically, it is found that interpolation in the variable $\ln (1-$ C.L.) is reliable, insofar that it varies approximately linearly with both mass difference and charged lepton mass in the neighborhood of the exclusion contours. The procedure used here is to interpolate along horizontal(fixed mass difference) and vertical(fixed charged mass) lines to find points that lie on the contour of $95 \%$ confidence level. There are additional interpolations along the curve defined by zero neutrino mass.

Once this set of points has been determined, circle fits are made to find the contour segments between points. For example, assume H, I, J, and $\mathrm{K}$ are sequential points along the contour. To find the segment between I and $\mathrm{J}$, two circle fits are 
Table 5.4. Average expected signal events and confidence levels of exclusion for Monte Carlo samples with mass differences of 0.2 and $0.4 \mathrm{GeV}$. Estimated errors on average expected numbers of events are systematic only; they do not include statistical fluctuations in observed numbers of events.

\begin{tabular}{|c|c|c|c|}
\hline$\delta \mathrm{M}(\mathrm{GeV})$ & $\mathrm{M}_{\mathrm{L}}(\mathrm{GeV})$ & Number of Events & C.L. $(\%)$ \\
\hline 0.2 & 1.0 & $6.5 \pm 1.1$ & 74.3 \\
\hline 0.2 & 2.0 & $11.8 \pm 1.9$ & 95.6 \\
\hline 0.2 & -3.0 & $11.2 \pm 1.8$ & 94.5 \\
\hline 0.2 & 4.0 & $10.0 \pm 1.6$ & 91.6 \\
\hline 0.2 & 5.0 & $-8.5 \pm 1.3$ & 86.4 \\
\hline 0.2 & 6.0 & ․ $7.8 \pm 1.2$ & 82.9 \\
\hline 0.2 & 8.0 & $4.4 \pm 0.7$ & 55.1 \\
\hline 0.2 & 10.0 & $2.2 \pm 0.4$ & 27.9 \\
\hline 0.2 & 11.0 & $1.1 \pm 0.2$ & 14.2 \\
\hline 0.2 & 12.0 & $0.6 \pm 0.1$ & 7.3 \\
\hline 0.3 & 0.3 & $0.5 \pm 0.2$ & 5.8 \\
\hline 0.3 & 1.0 & $24.2 \pm 3.5$ & $>99.9$ \\
\hline 0.3 & 2.0 & $42.8 \pm 6.2$ & $>99.9$ \\
\hline 0.3 & 3.0 & $36.0 \pm 5.2$ & $>99.9$ \\
\hline 0.3 & 4.0 & $33.5 \pm 4.9$ & $>99.9$ \\
\hline 0.3 & 5.0 & $29.0 \pm 4.2$ & $>99.9$ \\
\hline 0.3 & 6.0 & $25.2 \pm 3.7$ & $>99.9$ \\
\hline 0.3 & 8.0 & $16.3 \pm 2.4$ & 99.3 \\
\hline 0.3 & 10.0 & $8.6 \pm 1.3$ & 86.9 \\
\hline 0.3 & 11.0 & $6.0 \pm 0.9$ & 70.8 \\
\hline 0.3 & 12.0 & $3.6 \pm 0.5$ & 46.2 \\
\hline
\end{tabular}


performed. The circle(A) defined by points $\mathrm{H}, \mathrm{I}$, and $\mathrm{J}$ is determined, along with the circle(B) defined by $\mathrm{I}, \mathrm{J}$, and $\mathrm{K}$. In general, the two segments do not coincide exactly, but are close. To resolve the ambiguity, a weighted average of points along circles $\mathrm{A}$ and $B$ is taken, where the weights depend upon nearness to the endpoints $I$ and $J$, and upon the radii of curvature of the two circles. Specifically, for points. on the segment close to $\mathrm{I}$, circle $\mathrm{A}$ is given more weight than is circle $\mathrm{B}$. In addition, weighting the circle contributions according to their radii of curvature produces a smoother curve, avoiding rapid, unphysical variations.

The resulting contour for $95 \%$ confidence level is shown in fig. 5.22. As discussed in chapter 3 , the phase space suppression for producing very heavy leptons in association with an energetic photon severely limits this analysis for lepton masses greater than about $10 \mathrm{GeV}$. Along the bottom of the contour, at low mass differences, the total decay width becomes small enough that long lifetimes lead to reconstructed charged tracks with large impact parameters, for which trigger and reconstruction efficiencies are poor. At the bottom left, where the charged mass is very small, the sensitivity to very small mass differences is reduced because the visible energies in such cases can be substantial, leading to charged particles with momenta greater than the allowed $4 \mathrm{GeV}$.

One can construct an exclusion contour for $99 \%$ confidence level in exactly the same way. Figure 5.23 shows both the $95 \%$ and $99 \%$ c.l. contours, plotted logarithmically in mass difference. Because this analysis has been optimized to explore the region of very low mass difference, where previous experiments have been insensitive, increasing the required confidence level leads to rapid loss in the region excluded along the top of the contour where mass differences and therefore visible energies are relatively large. Along the bottom, however, the contours recede slowly with increasing confidence level, indicating that confidence level falls off very rapidly with decreasing mass difference. This is easily understood: the long charged lepton lifetimes associated with low mass differences are approximately inversely proportional to the cube 
Table 5.5. Confidence levels for exclusion of Monte Carlo heavy lepton samples with $(\delta \mathrm{M}=0.4,0.6)$

\begin{tabular}{|c|c|c|c|}
\hline$\delta \mathrm{M}(\mathrm{GeV})$ & $\mathrm{M}_{\mathrm{L}}(\mathrm{GeV})$ & Number of Events & C.L. (\%) \\
\hline 0.4 & 0.4 & $1.9 \pm 0.4$ & 24.9 \\
\hline 0.4 & 1.0 & $32.8 \pm 4.5$ & $>99.9$ \\
\hline 0.4 & 2.0 & $52.2 \pm 7.2$ & $>99.9$ \\
\hline 0.4 & -3.0 & $50.3 \pm 6.9$ & $>99.9$ \\
\hline 0.4 & 4.0 & $44.9 \pm 6.2$ & $>99.9$ \\
\hline 0.4 & 5.0 & $36.3 \pm 5.0$ & $>99.9$ \\
\hline 0.4 & 6.0 & $30.7 \perp 4.3$ & $>99.9$ \\
\hline 0.4 & 8.0 & $19.6 \pm 2.8$ & 99.8 \\
\hline 0.4 & 10.0 & $11.6 \pm 1.6$ & 95.5 \\
\hline 0.4 & 11.0 & $7.2 \pm 1.0$ & 79.4 \\
\hline 0.4 & 12.0 & $4.1 \pm 0.6$ & 51.9 \\
\hline 0.6 & 0.6 & $13.2 \pm 1.9$ & 97.5 \\
\hline 0.6 & 1.0 & $26.4 \pm 3.4$ & $>99.9$ \\
\hline 0.6 & 2.0 & $45.6 \pm 5.8$ & $>99.9$ \\
\hline 0.6 & 3.0 & $49.5 \pm 6.3$ & $>99.9$ \\
\hline 0.6 & 4.0 & $49.0 \pm 6.3$ & $>99.9$ \\
\hline 0.6 & 5.0 & $38.2 \pm 4.9$ & $>99.9$ \\
\hline 0.6 & 6.0 & $33.7 \pm 4.3$ & $>99.9$ \\
\hline 0.6 & 8.0 & $20.8 \pm 2.7$ & $>99.9$ \\
\hline 0.6 & 10.0 & $11.9 \pm 1.5$ & 96.1 \\
\hline 0.6 & 11.0 & $7.5 \pm 1.0$ & 81.7 \\
\hline 0.6 & 12.0 & $3.8 \pm 0.5$ & 49.0 \\
\hline
\end{tabular}


of the mass difference in this region. Consequently, the number of signal events that pass the $5 \mathrm{~cm}$ charged track impact parameter cut falls rapidly as one explores mass differences below $250 \mathrm{MeV}$.

\subsection{Conclusions and Outlook}

No evidence for the existence of a new close-mass lepton doublet has emerged from this work, permitting limits to be placed on allowed combinations of charged and neutral lepton masses. The novel requirement of an isolated photon has allowed exploration of extreme close-mass doublets previously inaccessible to experiment. The major limitation in exploring lower mass differences has been dependence upon a charged particle trigger. More progress could be made in future experiments with a trigger sensitive to the tagging photon itself. There would be technical difficulties in implementing such a trigger; backgrounds from cosmic rays and beam-associated backgrounds could be substantial, depending upon the detector and upon the accelerator supplying the electron and positron bearns. More difficulty would come from reconstructing the charged particle tracks, requiring sophisticated algorithms to detect and correctly interpret in-flight charged lepton decays. The rewards for such work would be the ability to detect the existence of not only close-mass leptons, but of general exotic processes characterized by very low visible energy due to undetected heavy particles.

The prospects for soon completely excluding close-mass leptons with masses below about $45 \mathrm{GeV}$ are quite good. Upcoming electron-positron experiments at centerof-mass energies equal to the $Z^{0}$ mass should allow determination of the total decay width of the $Z^{0}$ with sufficient accuracy to determine whether the $Z^{0}$ decays to various types of undiscovered particles. In particular, a new close-mass doublet with less than half the mass of the $\mathrm{Z}^{0}$ would increase the width because of the two decay modes $\mathrm{Z}^{0} \rightarrow \mathrm{L}^{+} \mathrm{L}^{-}$and $\mathrm{Z}^{0} \rightarrow \nu_{\mathrm{L}} \bar{\nu}_{\mathrm{L}}$. If experiments find that the total decay width is correctly predicted by the standard model with 3 generations, then it is straightfor- 
Table 5.6. Confidence levels for exclusion of Monte Carlo heavy lepton samples with $(\delta \mathrm{M}=0.8,1.0,1.2)$

\begin{tabular}{|c|c|c|c|}
\hline$\delta \mathrm{M}(\mathrm{GeV})$ & $\mathrm{M}_{\mathrm{L}}(\mathrm{GeV})$ & Number of Events & C.L. (\%) \\
\hline 0.8 & 0.8 & $16.5 \pm 2.2$ & 99.4 \\
\hline 0.8 & 1.0 & $17.9 \pm 2.3$ & 99.7 \\
\hline 0.8 & 2.0 & $30.7 \pm 4.0$ & $>99.9$ \\
\hline 0.8 & -3.0 & $37.2 \pm 4.9$ & $>99.9$ \\
\hline 0.8 & 4.0 & $40.5 \pm 5.4$ & $>99.9$ \\
\hline 0.8 & 5.0 & $34.0 \pm 4.6$ & $>99.9$ \\
\hline 0.8 & 6.0 & $28.6 \pm 3.9$ & $>99.9$ \\
\hline 0.8 & 8.0 & $19.7 \pm 2.7$ & 99.8 \\
\hline 0.8 & 10.0 & $10.6 \pm 1.4$ & 93.6 \\
\hline 0.8 & 11.0 & $6.6 \pm 0.9$ & 75.8 \\
\hline 0.8 & 12.0 & $3.6 \pm 0.5$ & 46.5 \\
\hline 1.0 & 1.0 & $13.8 \pm 1.2$ & 98.4 \\
\hline 1.0 & 2.0 & $20.9 \pm 2.0$ & $>99.9$ \\
\hline 1.0 & 3.0 & $28.1 \pm 2.7$ & $>99.9$ \\
\hline 1.2 & 1.2 & $12.8 \pm 1.7$ & 97.1 \\
\hline 1.2 & 2.0 & $17.9 \pm 2.5$ & 99.6 \\
\hline 1.2 & 3.0 & $25.8 \pm 3.5$ & $>99.9$ \\
\hline 1.2 & 4.0 & $27.8 \pm 3.7$ & $>99.9$ \\
\hline 1.2 & 5.0 & $25.3 \pm 3.5$ & $>99.9$ \\
\hline 1.2 & 6.0 & $24.1 \pm 3.3$ & $>99.9$ \\
\hline 1.2 & 8.0 & $17.1 \pm 2.4$ & 99.5 \\
\hline 1.2 & 10.0 & $9.2 \pm 1.3$ & 89.2 \\
\hline 1.2 & 11.0 & $6.0 \pm 0.8$ & 70.4 \\
\hline 1.2 & 12.0 & $3.1 \pm 0.4$ & 40.3 \\
\hline
\end{tabular}


Table 5.7. Confidence levels for exclusion of Monte Carlo heavy lepton samples with $(\delta \mathrm{M}=1.4,1.6,1.8,2.0)$

\begin{tabular}{|c|c|c|c|}
\hline$\delta \mathrm{M}(\mathrm{GeV})$ & $\mathrm{M}_{\mathrm{L}}(\mathrm{GeV})$ & Number of Events & C.L. $(\%)$ \\
\hline 1.4 & 3.0 & $17.8 \pm 1.6$ & 99.8 \\
\hline 1.4 & 4.0 & $20.9 \pm 1.9$ & $>99.9$ \\
\hline 1.4 & 5.0 & $21.1 \pm 1.9$ & $>99.9$ \\
\hline $1.4-$ & 6.0 & $18.8 \pm 2.5$ & 99.8 \\
\hline 1.6 & 2.0 & $12.4 \pm 1.7$ & 96.7 \\
\hline 1.6 & 3.0 & $13.9 \pm 1.8$ & 98.2 \\
\hline 1.6 & 4.0 & $15.8 \pm 2.1$ & 99.2 \\
\hline 1.6 & 5.0 & $15.5 \pm 2.1$ & 99.0 \\
\hline 1.6 & 6.0 & $15.7 \pm 2.0$ & 99.1 \\
\hline 1.6 & 8.0 & $12.1 \pm 1.6$ & 96.4 \\
\hline 1.6 & 10.0 & $7.3 \pm 1.0$ & 80.2 \\
\hline 1.6 & 11.0 & $4.7 \pm 0.6$ & 58.2 \\
\hline 1.6 & 12.0 & $2.5 \pm 0.3$ & 32.4 \\
\hline 1.8 & 4.0 & $12.5 \pm 1.1$ & 97.2 \\
\hline 1.8 & 5.0 & $14.0 \pm 1.8$ & 98.2 \\
\hline 1.8 & 6.0 & $14.0 \pm 1.8$ & 98.3 \\
\hline 1.8 & 8.0 & $10.3 \pm 1.4$ & 92.8 \\
\hline 2.0 & 5.0 & $11.1 \pm 1.0$ & 95.0 \\
\hline 2.0 & 6.0 & $10.1 \pm 0.9$ & 92.8 \\
\hline
\end{tabular}

ward to exclude a fourth generation lepton doublet. On the other hand, if the width is larger than predicted, determining the nature of implied new particle(s) could be a challenge.

If the new process were hidden in two-photon backgrounds because of large miss- 


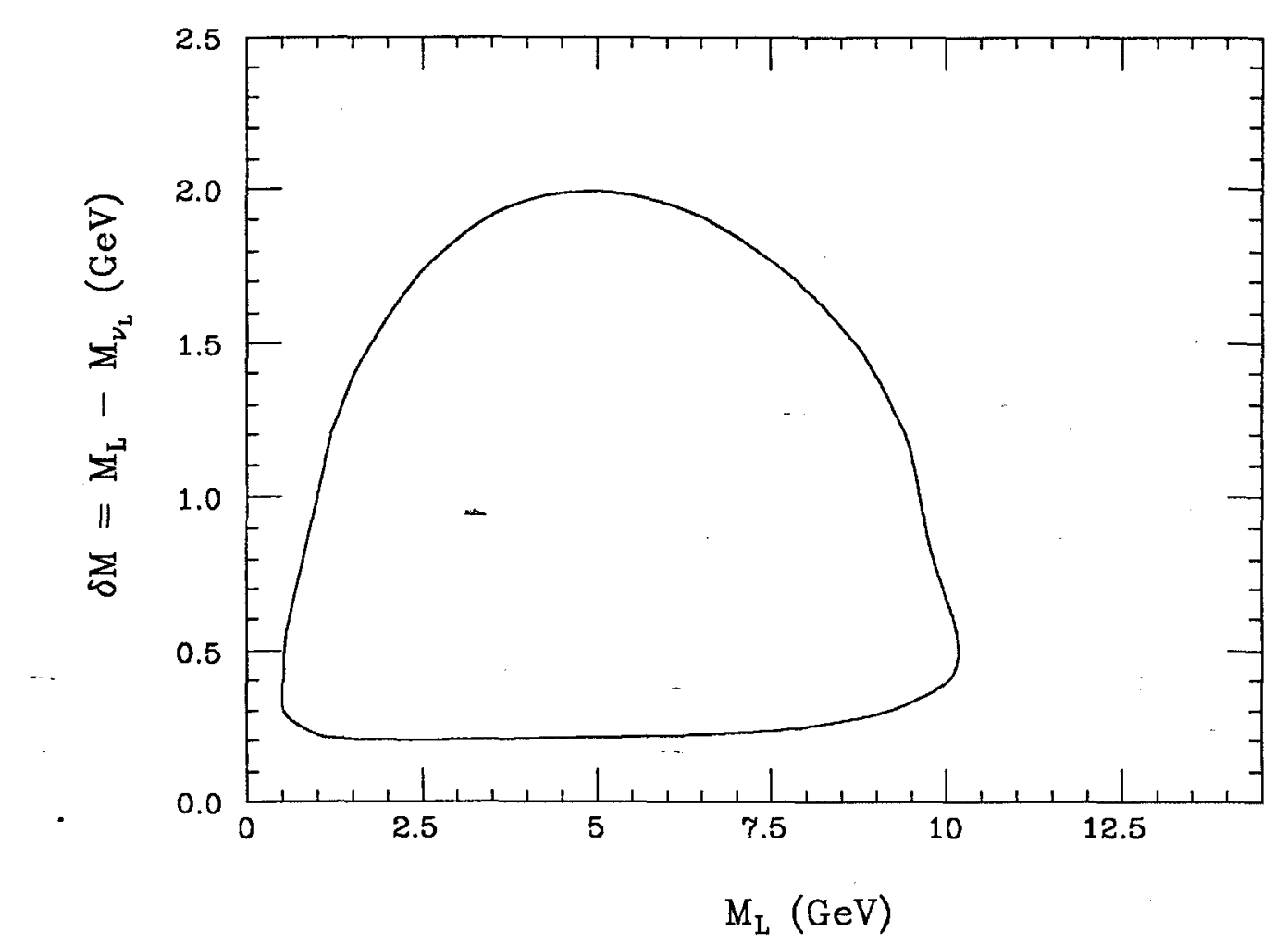

Figure 5.22. Exclusion contour at $95 \%$ confidence level

ing energy, as in the case of close-mass doublets, it might be difficult to extract the signal. In such a case, photon-tagging could again become useful. A promising approach would be that proposed for neutrino counting with photon-tagging, ${ }^{69}$ that is, to collide the electron-positron beams together at a center-of-mass energy several $\mathrm{GeV}$ above the $\mathrm{Z}^{0}$ resonance. For processes associated with the virtual $\mathrm{Z}^{0}$ channel, this produces a photon energy spectrum that reflects the $\mathrm{Z}^{0}$ resonance, as shown in fig. 5.24. For the figure, a $Z^{0}$ mass of $93 \mathrm{GeV}$ and a center-of-mass energy of 97 $\mathrm{GeV}$ have been assumed. The histograms are calculations of the radiatively corrected differential cross sections for the four charged lepton masses shown.* Therefore, one signal would be very low energy events with a tagging photon spectrum like that

* The radiative corrections to the bremsstrahlung process have been calculated with a Monte Carlo program ${ }^{70}$ that uses a structure function formalism and an evolution algorithm to simulate multiple photon emission. My thanks to Giovanni Bonvicini for providing a preliminary version of the program. 


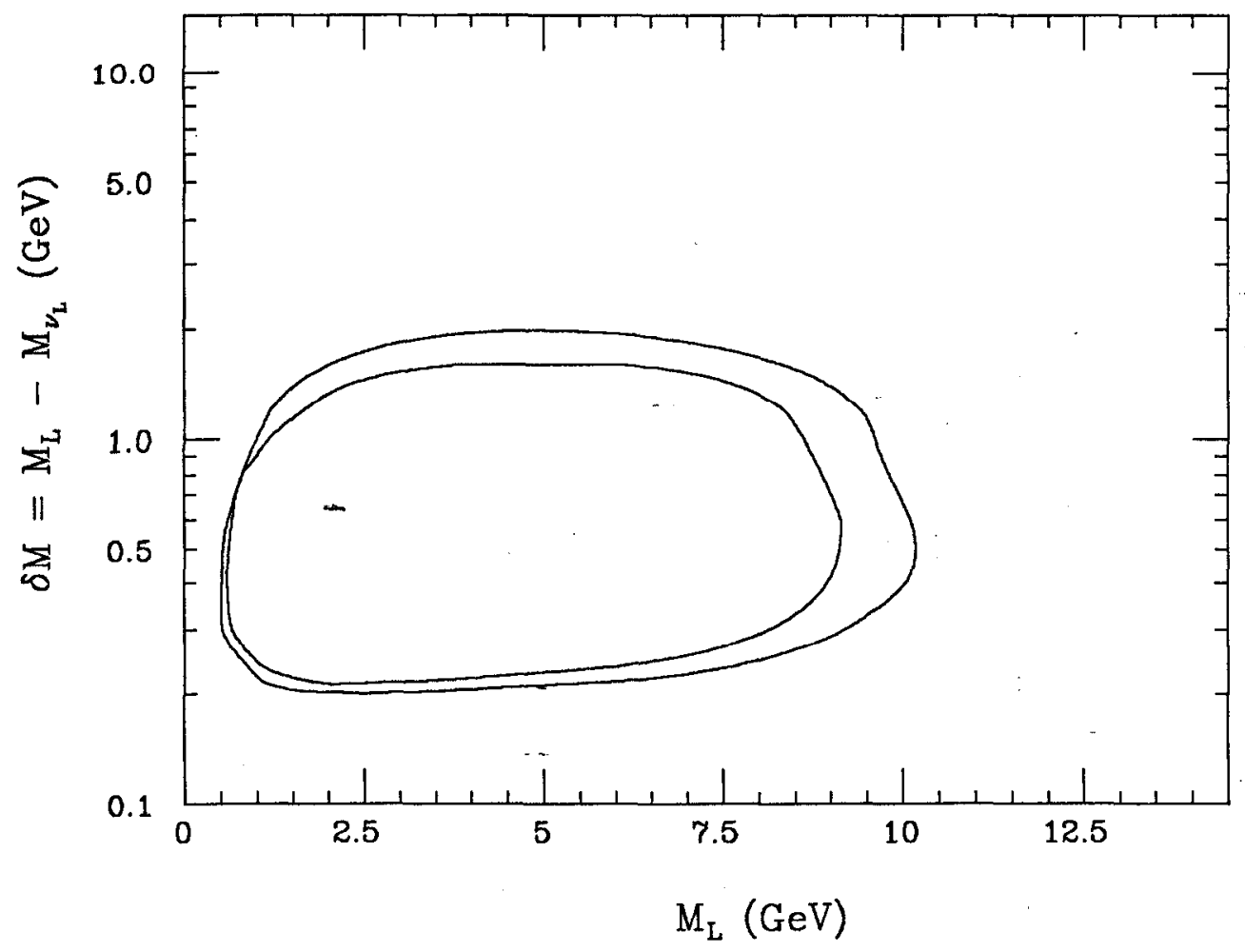

Figure 5.23. Exclusion contours at $95 \%$ and $99 \%$ confidence levels.

depicted. The tagging photon requirement would still suppress two-photon backgrounds, which are less important at the $\mathrm{Z}$ resonance, and remaining backgrounds could be removed with additional selection cuts, such as those described in this chapter. Hopefully, nature is not as perverse as to give us fourth generation leptons so difficult to find and identify, but if she should, radiative tagging provides a powcrful tool. 


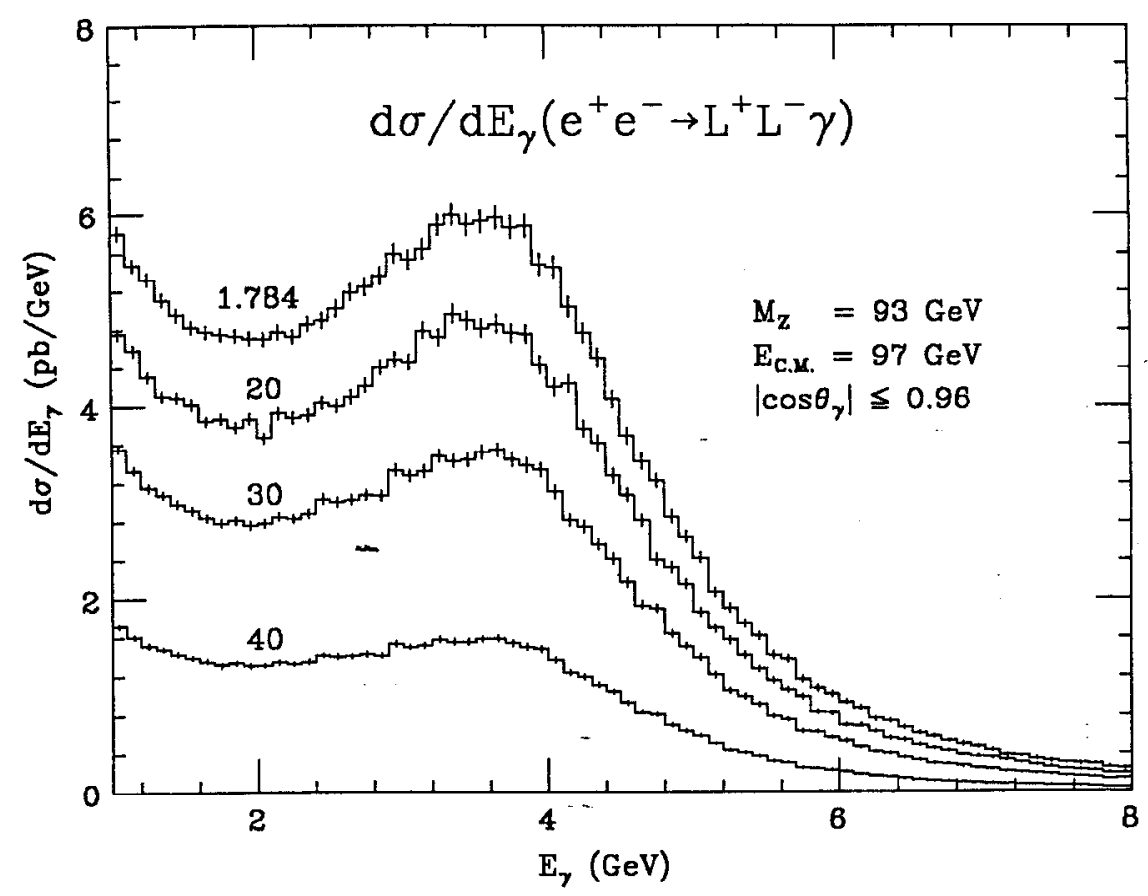

Figure 5.24. Photon energy spectrum expected for charged lepton production at a center-of-mass energy $4 \mathrm{GeV}$ above the $Z^{0}$ mass. 


\section{Appendix A}

\section{Lepton Production and Decay Simulation}

To estimate the efficiency for detecting a new heavy sequential lepton, one must -accurately simulate the lepton's production and decay. For this analysis, the simulation was performed with a FORTRAN program called LULEPT, written by David Stoker. ${ }^{71}$ Because the analysis depends critically on the accuracy of the LULEPT simulation, this appendix describes in some detail the formulas LULEPT uses and the assumptions it makes.

The algorithm accounts for annihilation production through both the $\gamma$ and $\mathrm{Z}^{0}$ channels, including interference terms. Lowest order initial state radiation from the electron and positron is also simulated, as described in chapter 3. Spin effects, including the spin-spin correlation, are treated properly in production and decay calculations. LULEPT explicitly simulates the following decays:

$$
\mathrm{L}^{-} \rightarrow \nu_{\mathrm{L}} \mathrm{e}^{-} \bar{\nu}_{\mathrm{e}}
$$

$$
\mathrm{L}^{-} \rightarrow \nu_{\mathrm{L}} \mu^{-} \bar{\nu}_{\mu}
$$

$$
\mathrm{L}^{-} \rightarrow \nu_{\mathrm{L}} \pi^{-}
$$

$$
\mathrm{L}^{-} \rightarrow \nu_{\mathrm{L}} \rho^{-}
$$

$$
\mathrm{L}^{-} \rightarrow \nu_{\mathrm{L}} \mathrm{K}^{-}
$$

$$
\mathrm{L}^{-} \rightarrow \nu_{\mathrm{L}} \mathrm{K}^{*-}
$$

$$
\mathrm{L}^{-} \rightarrow \nu_{\mathrm{L}} \mathrm{a}_{1}^{-}
$$


[8]

$[9]$

$[10]$

$$
\begin{array}{ll}
\mathrm{L}^{-} \rightarrow \nu_{\mathrm{L}} \overline{\mathrm{u}} \mathrm{d} & \left(\mathrm{M}_{\mathrm{L}-}>\mathrm{M}_{\nu_{\mathrm{L}}}+1.275 \mathrm{GeV}\right) \\
\mathrm{L}^{-} \rightarrow \nu_{\mathrm{L}} \overline{\mathrm{cs}} & \left(\mathrm{M}_{\mathrm{L}-}>\mathrm{M}_{\nu_{\mathrm{L}}}+2.0 \mathrm{GeV}\right) \\
\mathrm{L}^{-} \rightarrow \nu_{\mathrm{L}} \tau^{-} \bar{\nu}_{\tau} & \left(\mathrm{M}_{\mathrm{L}}>\mathrm{M}_{\nu_{\mathrm{L}}}+1.784 \mathrm{GeV}\right)
\end{array}
$$

where $M_{L}$ and $M_{\nu_{L}}$ are the charged and neutral lepton masses.

Depending on the mass difference between $\mathrm{L}^{-}$and $\nu_{\mathrm{L}}$, some of these processes may be forbidden by kinematics. The partial widths are fairly straightforward to calculate, except for reactions[8-9], which require a fragmentation scheme to convert the initial quarks into visible hadrons. This fragmentation is performed by the LUND Monte Carlo program. ${ }^{42}$

LULEPT was designed to allow arbitrary admixtures of $(\mathrm{V}-\mathrm{A})$ and $(\mathrm{V}+\mathrm{A})$ couplings at the $\mathrm{L} \nu_{\mathrm{L}} \mathrm{W}$ vertex. The program also allows longitudinal polarization of the initial electrons and positrons. For this analysis, however, the current is assumed to be pure $(\mathrm{V}-\mathrm{A})$, and initial longitudinal polarization zero.

\section{A.1 Production Simulation}

In general, the differential cross section for $\mathrm{e}^{+} \mathrm{e}^{-}$annihilation into two spin $\frac{1}{2}$ charged leptons is

$$
d \sigma=d \sigma_{0}\left(1+\mathrm{c}_{\mu} \mathrm{S}^{\mu}+\overline{\mathrm{c}}_{\mu} \overline{\mathrm{S}}^{\mu}+\mathrm{c}_{\mu \nu} \mathrm{S}^{\mu} \overline{\mathrm{S}}^{\nu}\right)
$$

where $d \sigma_{0}$ is the spin-averaged differential cross section, and $\mathrm{S}$ and $\overline{\mathrm{S}}$ are 4 -vectors describing the spin of the $\mathrm{L}^{-}$and the $\mathrm{L}^{+}$particles:

$$
\left.S=\dot{S}_{0}, \vec{S}_{L^{-}}\right)
$$

In the rest frame of either lepton, the time component of its spin 4-vector vanishes:

$$
S^{\prime}=(0, \hat{S})
$$


The differential decay rates for the two leptons are in general

$$
\begin{aligned}
& d \Gamma_{\mathrm{L}^{-}}=d \Gamma_{\mathrm{L}-}^{0}\left(1+\mathrm{b}_{\mu} \mathrm{S}^{\mu}\right) \\
& d \Gamma_{\mathrm{L}^{+}}=d \Gamma_{\mathrm{L}+}^{0}\left(1+\overline{\mathrm{b}}_{\mu} \overline{\mathrm{S}}^{\mu}\right)
\end{aligned}
$$

where $\mathrm{P}_{\mathrm{L}^{-}}, \mathrm{P}_{\mathrm{L}^{+}}$are the 4-momenta of the heavy leptons, where $\mathrm{b} \cdot \mathrm{P}_{\mathrm{L}^{-}}=\overline{\mathrm{b}} \cdot \mathrm{P}_{\mathrm{L}^{+}}=0$, and where $d \Gamma^{0}$ is the spin-averaged decay width for each lepton. It can be shown ${ }^{72}$ that these equations lead-to a combined differential cross section for production and decay given by

$$
d \sigma=4 d \sigma_{0}\left(1-\mathrm{c}_{\mu} \mathrm{b}^{\mu}-\overline{\mathrm{c}}_{\boldsymbol{\mu}} \overline{\mathrm{b}}^{\mu}+\mathrm{c}_{\mu \nu} \mathrm{b}^{\mu} \overline{\mathrm{b}}^{\nu}\right) \frac{d \Gamma_{\mathrm{L}}-d \Gamma_{\mathrm{L}}+}{\Gamma_{\mathrm{L}}-\Gamma_{\mathrm{L}}+}
$$

where $4 d \sigma_{0}$ is the total cross section summed over final state charged lepton spins. From this expression it is clear that production and decay calculations cannot be factorized. Not only are the spins of the leptons correlated, and thus the kinematics of their decay products correlated, but the differential cross sections itself depends on that correlation.

Following the notation of $\mathrm{Ward}^{72}$, one can write

$$
\frac{d \sigma\left(\mathrm{e}^{+} \mathrm{e}^{-} \rightarrow \mathrm{L}^{-} \mathrm{L}^{+}\right)}{d \Omega}=\frac{\alpha^{2}}{16 \mathrm{~s}} \beta|\mathrm{F}|^{2}
$$

where $d \Omega$ is the differential solid angle element of $\mathrm{L}^{-}$in the $\mathrm{e}^{+} \mathrm{e}^{-}$c.m. frame, and $\beta$ gives the magnitude of each lepton's velocity:

$$
\beta=\frac{\left|\overrightarrow{\mathrm{p}}_{\mathrm{L}}\right|}{\mathrm{E}_{\mathrm{L}-}}=\frac{\left|\overrightarrow{\mathrm{p}}_{\mathrm{L}+}\right|}{\mathrm{E}_{\mathrm{L}+}}
$$

$|\mathrm{F}|^{2}$ is defined by

$$
|\mathrm{F}|^{2}=\mathrm{C}+\mathrm{C}_{\mu} \mathrm{S}^{\mu}+\overline{\mathrm{C}}_{\mu} \overline{\mathrm{S}}^{\mu}+\mathrm{C}_{\mu \nu} \mathrm{S}^{\mu} \overline{\mathrm{S}}^{\nu}
$$


The coefficients are given by

$$
\begin{aligned}
& \mathrm{C}=\mathrm{e}_{\mathrm{f}}^{2}\left(1+\cos ^{2} \theta+\frac{\sin ^{2} \theta}{\gamma^{2}}\right) \\
& +\frac{\mathrm{e}_{\mathrm{f}}}{\sin ^{2} \theta_{\mathrm{W}} \cos ^{2} \theta_{\mathrm{W}}} \frac{\mathrm{s}\left(\mathrm{s}-\mathrm{M}_{\mathrm{Z}}^{2}\right)}{\left(\mathrm{s}-\mathrm{M}_{\mathrm{Z}}^{2}\right)^{2}+\mathrm{M}_{\mathrm{Z}}^{2} \Gamma_{\mathrm{Z}}^{2}} \\
& \times\left\{-2 \mathrm{v}_{\mathbf{f}} \mathrm{v}_{\mathrm{e}}\left(1+\cos ^{2} \theta+\frac{\sin ^{2} \theta}{\gamma^{2}}\right)+\mathrm{a}_{\mathfrak{f}} \beta \cos \theta\right\} \\
& +\frac{1}{\sin ^{4} \theta_{\mathrm{W}} \cos ^{4} \theta_{\mathrm{W}}} \frac{\mathrm{s}^{2}}{\left(\mathrm{~s}-\mathrm{M}_{\mathrm{Z}}^{2}\right)^{2}+\mathrm{M}_{\mathrm{Z}}^{2} \Gamma_{\mathrm{Z}}^{2}} \\
& \times\left\{\left(v_{\mathrm{e}}^{2}+\frac{1}{16}\right)\left[\left(\mathrm{v}_{\mathrm{f}}^{2}+\mathrm{a}_{\mathrm{f}}^{2}\right)\left(1+\beta^{2} \cos ^{2} \theta\right)+\frac{1}{\gamma^{2}}\left(\mathrm{v}_{\mathrm{f}}^{2}-\mathrm{a}_{\mathrm{f}}^{2}\right)\right]\right. \\
& \left.-2 \mathrm{v}_{\mathrm{f}} \mathrm{a}_{\mathrm{f}} \mathrm{v}_{\mathrm{e}} \beta \cos \theta\right\} \\
& \mathrm{C}_{\mu}=\frac{\mathrm{e}_{\mathrm{f}}}{\sin ^{2} \theta_{\mathrm{W}} \cos ^{2} \theta_{\mathrm{W}}} \frac{2 \mathrm{M}_{\mathrm{L}}}{\left(\mathrm{s}-\mathrm{M}_{\mathrm{Z}}^{2}\right)^{2}+\mathrm{M}_{\mathrm{Z}}^{2} \Gamma_{\mathrm{Z}}^{2}} \\
& \times\left\{\left(\mathrm{s}-\mathrm{M}_{\mathrm{Z}}^{2}\right)\left[\mathrm{v}_{\mathrm{f}}\left(\mathrm{p}_{\mathrm{e}}-\mathrm{p}_{\overline{\mathrm{e}}}\right)_{\mu}+2 \mathrm{a}_{\mathrm{f}} \mathrm{v}_{\mathrm{e}}\left\{\left(\mathrm{p}_{\mathrm{e}}+\mathrm{p}_{\overline{\mathrm{e}}}\right)_{\mu}-\beta \cos \theta\left(\mathrm{p}_{\mathrm{e}}-\mathrm{p}_{\overline{\mathrm{e}}}\right)_{\mu}\right\}\right]\right. \\
& \left.-\frac{2 \mathrm{M}_{\mathrm{Z}} \Gamma_{\mathrm{Z}}}{s} \mathrm{a}_{\mathrm{f}} \epsilon_{\mu}{ }^{\alpha \beta \gamma} \mathrm{p}_{\bar{e}_{\alpha}} \mathrm{p}_{\boldsymbol{\beta} \beta} \mathrm{p}_{\mathrm{e}_{\gamma}}\right\} \\
& +\frac{1}{\sin ^{4} \theta_{\mathrm{W}} \cos ^{4} \theta_{\mathrm{W}}} \frac{\mathrm{s}^{2}}{\left(\mathrm{~s}-\mathrm{M}_{\mathrm{Z}}^{2}\right)^{2}+\mathrm{M}_{\mathrm{Z}}^{2} \Gamma_{\mathrm{Z}}^{2}} \frac{1}{4 \mathrm{M}_{\mathrm{L}} \gamma^{2}} \\
& \times\left\{-4 \mathrm{v}_{\mathrm{f}} \mathrm{a}_{\mathrm{f}}\left(\mathrm{v}_{\mathrm{e}}^{2}+\frac{1}{16}\right)\left[\left(\mathrm{p}_{\mathrm{e}}+\mathrm{p}_{\overline{\mathrm{e}}}\right)_{\mu}-\beta \cos \theta\left(\mathrm{p}_{\mathrm{e}}-\mathrm{p}_{\overline{\mathrm{e}}}\right)_{\mu}\right]\right. \\
& \left.-2 \mathrm{v}_{\mathrm{e}}\left[\mathrm{v}_{\mathrm{f}}^{2}\left(\mathrm{p}_{\mathrm{e}}-\mathrm{p}_{\overline{\mathrm{e}}}\right)_{\mu}-\mathrm{a}_{\mathrm{f}}^{2} \beta \cos \theta\left(\mathrm{p}_{\mathrm{e}}+\mathrm{p}_{\overline{\mathrm{e}}}\right)_{\mu}\right]\right\} \\
& \overline{\mathrm{C}}_{\mu}=\frac{\mathrm{e}_{\mathrm{f}}}{\sin ^{2} \theta_{\mathrm{W}} \cos ^{2} \theta_{\mathrm{W}}} \frac{2 \mathrm{M}_{\mathrm{L}}}{\left(\mathrm{s}-\mathrm{M}_{\mathrm{Z}}^{2}\right)^{2}+\mathrm{M}_{\mathrm{Z}}^{2} \Gamma_{\mathrm{Z}}^{2}} \\
& \times\left\{\left(\mathrm{s}-\mathrm{M}_{\mathrm{Z}}^{2}\right)\left[\mathrm{v}_{\mathrm{f}}\left(\mathrm{p}_{\mathrm{e}}-\mathrm{p}_{\overline{\mathrm{e}}}\right)_{\mu}-2 \mathrm{a}_{\mathrm{f}} \mathrm{v}_{\mathrm{e}}\left\{\left(\mathrm{p}_{\mathrm{e}}+\mathrm{p}_{\overline{\mathrm{e}}}\right)_{\mu}+\beta \cos \theta\left(\mathrm{p}_{\mathrm{e}}-\mathrm{p}_{\overline{\mathrm{e}}}\right)_{\mu}\right\}\right]\right. \\
& \left.+\frac{2 \mathrm{M}_{\mathrm{Z}} \Gamma_{\mathrm{Z}}}{s} \mathrm{a}_{\mathrm{f}} \epsilon_{\mu}{ }^{\alpha \beta \gamma} \mathrm{p}_{\bar{e}_{\alpha}} \mathrm{p}_{\overline{\mathrm{f}} \beta} \mathrm{p}_{\mathrm{e}_{\gamma}}\right\} \\
& +\frac{1}{\sin ^{4} \theta_{\mathrm{W}} \cos ^{4} \theta_{\mathrm{W}}} \frac{\mathrm{s}^{2}}{\left(\mathrm{~s}-\mathrm{M}_{\mathrm{Z}}^{2}\right)^{2}+\mathrm{M}_{\mathrm{Z}}^{2} \Gamma_{\mathrm{Z}}^{2}} \frac{1}{4 \mathrm{M}_{\mathrm{L}} \gamma^{2}} \\
& \times\left\{4 \mathrm{v}_{\mathrm{f}} \mathrm{a}_{\mathrm{f}}\left(\mathrm{v}_{\mathrm{e}}^{2}+\frac{1}{16}\right)\left[\left(\mathrm{p}_{\mathrm{e}}+\mathrm{p}_{\overline{\mathrm{e}}}\right)_{\mu}+\beta \cos \theta\left(\mathrm{p}_{\mathrm{e}}-\mathrm{p}_{\overline{\mathrm{e}}}\right)_{\mu}\right]\right. \\
& \left.-2 \mathrm{v}_{\mathrm{e}}\left[\mathrm{v}_{\mathrm{f}}^{2}\left(\mathrm{p}_{\mathrm{e}}-\mathrm{p}_{\overline{\mathrm{e}}}\right)_{\mu}+\mathrm{a}_{\mathrm{f}}^{2} \beta \cos \theta\left(\mathrm{p}_{\mathrm{e}}+\mathrm{p}_{\overline{\mathrm{e}}}\right)_{\mu}\right]\right\}
\end{aligned}
$$




$$
\begin{aligned}
& C_{\mu \nu}=e_{\mathrm{f}}^{2}\left\{\beta^{2} \sin ^{2} \theta \mathrm{g}_{\mu \nu}-\frac{4}{s}\left(\mathrm{p}_{\mathrm{e}_{\mu}} \mathrm{p}_{\overline{\mathrm{e}}_{\nu}}+\mathrm{p}_{\mathrm{e}_{\nu}} \mathrm{p}_{\overline{\mathrm{e}}_{\mu}}\right)-\frac{4 \beta \cos \theta}{s}\left(\mathrm{p}_{\mathrm{e}_{\mu}} \mathrm{p}_{\overline{\mathrm{e}}_{\nu}}-\mathrm{p}_{\mathrm{e}_{\nu}} \mathrm{p}_{\overline{\mathrm{e}}_{\mu}}\right)\right\} \\
& +\frac{e_{f}}{\sin ^{2} \theta_{W} \cos ^{2} \theta_{W}} \frac{1}{\left(s-M_{Z}^{2}\right)^{2}+M_{Z}^{2} \Gamma_{Z}^{2}} \\
& \times\left\{( \mathrm { s } - \mathrm { M } _ { \mathrm { Z } } ^ { 2 } ) \left[-2 \mathrm{v}_{\mathrm{f}} \mathrm{v}_{\mathrm{e}} s \beta^{2} \sin ^{2} \theta \mathrm{g}_{\mu \nu}+8 \mathrm{v}_{\mathrm{f}} \mathrm{v}_{\mathrm{e}}\left(\mathrm{p}_{\mathrm{e}_{\mu}} \mathrm{p}_{\overline{\mathrm{e}}_{\nu}}+\mathrm{p}_{\mathrm{e}_{\nu}} \mathrm{p}_{\overline{\mathrm{e}}_{\mu}}\right)\right.\right. \\
& \left.+\left(\frac{1}{\gamma^{2}} a_{f}+8 v_{f} v_{e} \beta \cos \theta\right)\left(p_{e_{\mu}} p_{\bar{e}_{\nu}}-p_{e_{\nu}} p_{\bar{e}_{\mu}}\right)\right] \\
& +4 \mathrm{M}_{\mathrm{Z}} \bar{\Gamma}_{\mathrm{Z}} a_{\mathrm{f}} \mathrm{v}_{\mathrm{e}}\left[\epsilon_{\mu \nu}{ }^{\alpha \beta} \mathrm{p}_{\mathrm{f} \alpha} \mathrm{p}_{\overline{\mathrm{f}} \beta}-\beta \cos \theta \epsilon_{\mu \nu}{ }^{\alpha \beta}\left(\mathrm{p}_{\mathrm{e}}-\mathrm{p}_{\overline{\mathrm{e}}}\right)_{\alpha} \mathrm{p}_{\overline{\mathrm{f}} \beta}\right. \\
& +\frac{1}{\mathrm{~s}}\left(\mathrm{p}_{\mathrm{e}}-\mathrm{p}_{\overline{\mathrm{e}}}\right)_{\mu} \epsilon_{\nu}{ }^{\alpha \beta \gamma} \\
& \left.\left.\times\left(\mathrm{p}_{\mathrm{e}}-\mathrm{p}_{\overline{\mathrm{e}}}\right)_{\alpha}\left(\mathrm{p}_{\mathrm{e}}+\mathrm{p}_{\overline{\mathrm{e}}}\right)_{\beta}\left(\mathrm{p}_{\mathrm{f}}-\mathrm{p}_{\overline{\mathrm{f}}}\right)_{\gamma}\right]\right\} \\
& +\frac{1}{\sin ^{4} \theta_{\mathrm{W}} \cos ^{4} \theta_{\mathrm{W}}} \frac{\mathrm{s}}{\left(\mathrm{s}-\mathrm{M}_{\mathrm{Z}}^{2}\right)^{2}+\mathrm{M}_{\mathrm{Z}}^{2} \Gamma_{\mathrm{Z}}^{2}} \\
& \times\left\{\left(\mathrm{v}_{\mathrm{f}}^{2}-\mathrm{a}_{\mathrm{f}}^{2}\right)\left(\mathrm{v}_{\mathrm{e}}^{2}+\frac{1}{16}\right) \mathrm{s} \beta^{2} \sin ^{2} \theta \mathrm{g}_{\mu \nu}\right. \\
& -4\left(\mathrm{v}_{\mathrm{f}}^{2}-\beta^{2} \mathrm{a}_{\mathrm{f}}^{2}\right)\left(\mathrm{v}_{\mathrm{e}}^{2}+\frac{1}{16}\right)\left(\mathrm{p}_{\mu} \mathrm{p}_{\overline{\mathrm{e}}_{\nu}}+\mathrm{p}_{\mathrm{e}_{\nu}} \mathrm{p}_{\overline{\mathrm{e}}_{\mu}}\right)
\end{aligned}
$$

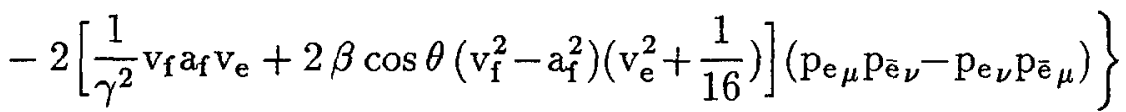

where $e_{f}$ is the lepton charge $\left(e_{f}=-1\right), v_{e}$ is the vector coupling of the electron, $a_{f}$ and $v_{f}$ are the axial and vector couplings of the lepton. $M_{Z}$ and $\Gamma_{Z}$ are the mass and total width of the $\mathrm{Z}$ boson, as given in chapter 3 . In this analysis, it is assumed $\mathrm{a}_{\mathrm{e}}=\mathrm{a}_{\mathrm{f}}=-\frac{1}{4} ; \mathrm{v}_{\mathrm{e}}=\mathrm{v}_{\mathrm{f}}=-\frac{1}{4}+\sin ^{2} \theta_{\mathrm{W}} ;$ and $\sin ^{2} \theta_{\mathrm{W}}=0.22$. The Lorentz boost factor is $\gamma=1 / \sqrt{1-\beta^{2}}$. The four-momenta, $\mathrm{p}_{\mathrm{e}}, \mathrm{p}_{\overline{\mathrm{e}}}, \mathrm{p}_{\mathrm{f}}$, and $\mathrm{p}_{\overline{\mathrm{f}}}$, refer to the electron, positron, $\mathrm{L}^{-}$, and $\mathrm{L}^{+}$, respectively, where $\theta$ is the angle between the electron and $\mathrm{L}^{-}$. The antisymmetric tensor $\epsilon_{\alpha \beta \gamma \delta}$ is defined by

$$
\epsilon_{\alpha \beta \gamma \delta}=\left\{\begin{aligned}
1 & \text { even permutation of }[\alpha=\mathrm{t}, \beta=\mathrm{x}, \gamma=\mathrm{y}, \delta=\mathrm{z}] \\
-1 & \text { odd permutation of }[\alpha=\mathrm{t}, \beta=\mathrm{x}, \gamma=\mathrm{y}, \delta=\mathrm{z}] \\
0 & \text { otherwise }
\end{aligned}\right.
$$

where $t, x, y$, and $z$ are the time and space coordinates. 
One needs to evaluate eqn. A.2 in some reference frame. The most convenient frame in which to do this is the $\mathrm{e}^{+} \mathrm{e}^{-}$c.m. frame. A coordinate system is defined in the $\mathrm{e}^{+} \mathrm{e}^{-}$c.m. frame (see fig. A.1) where the $\mathrm{L}^{-}$direction is along the positive $\mathrm{z}$-axis, and the initial electron direction is rotated an angle $\theta$ away from the $\mathrm{z}$-axis in the $x-z$ plane. In each heavy lepton's rest frame one has

$$
\begin{array}{r}
\mathrm{S}^{\prime}=\left(0, \hat{\mathrm{S}}^{\prime}\right) \\
-\quad \overline{\mathrm{S}}^{\prime}=\left(0, \hat{\overline{\mathrm{S}}}^{\prime}\right)
\end{array}
$$

which means that in the $\mathrm{e}^{+} \mathrm{e}^{-}$c.m. frame,

$$
\begin{aligned}
& \mathrm{S}=\left(\gamma \beta \mathrm{S}_{\mathrm{z}}^{\prime}, \mathrm{S}_{\mathrm{x}}^{\prime}, \mathrm{S}_{\mathrm{y}}^{\prime}, \gamma \mathrm{S}_{\mathrm{z}}^{\prime}\right) \\
& \overline{\mathrm{S}}=\left(-\gamma \beta \overline{\mathrm{S}}_{\mathrm{z}}^{\prime}, \overline{\mathrm{S}}_{\mathrm{x}}^{\prime}, \overline{\mathrm{S}}_{\mathrm{y}}^{\prime}, \gamma \overline{\mathrm{S}}_{\mathrm{z}}^{\prime}\right)
\end{aligned}
$$

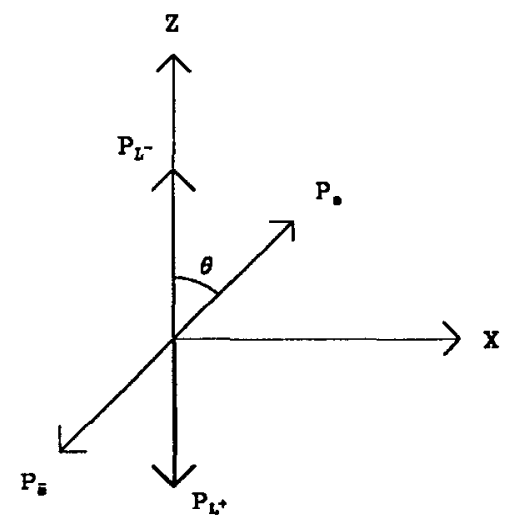

Figure A.1. The coefficients $\mathrm{C}_{\mathrm{i}}^{\prime}$ and $\mathrm{C}_{\mathrm{ij}}^{\prime}$ are evaluated in the c.m. frame of the $\mathrm{L}^{-}-\mathrm{L}^{+}$pair where $\theta$ is the angle between the $\mathrm{L}^{-}$and initial $\mathrm{e}^{-}$directions.

For PEP energies, one has $\mathrm{M}_{e} \ll \mathrm{E}$, allowing the approximation:

$$
\begin{aligned}
& \mathrm{p}_{\mathrm{e}}=(\mathrm{E}, \mathrm{E} \sin \theta, 0, \mathrm{E} \cos \theta) \\
& \mathrm{p}_{\overline{\mathrm{e}}}=(\mathrm{E},-\mathrm{E} \sin \theta, 0,-\mathrm{E} \cos \theta)
\end{aligned}
$$


while

$$
\begin{aligned}
& \mathrm{p}_{\mathrm{f}}=(\mathrm{E}, 0,0, \beta \mathrm{E}) \\
& \mathrm{p}_{\overline{\mathrm{f}}}=(\mathrm{E}, 0,0,-\beta E)
\end{aligned}
$$

One can define new coefficients, $\mathrm{C}_{\mathrm{i}}^{\prime}, \overline{\mathrm{C}}_{\mathrm{i}}^{\prime}$, and $\mathrm{C}_{\mathrm{ij}}^{\prime}$, which specify the correlation between the rest-frame components of the two leptons:

$$
\begin{gathered}
\sum_{i=\mathrm{x}, \mathrm{y}, \mathrm{z}} \mathrm{C}_{\mathrm{i}}^{\prime} \mathrm{S}_{\mathrm{i}}^{\prime} \equiv \mathrm{C}_{\mu} \mathrm{S}^{\mu} \\
\sum_{i=\mathrm{x}, \mathrm{y}, \mathrm{z}} \overline{\mathrm{C}}_{\mathrm{i}}^{\prime} \overline{\mathrm{S}}_{\mathrm{i}}^{\prime} \equiv \overline{\mathrm{C}}_{\mu} \overline{\mathrm{S}}^{\mu} \\
\sum_{i, j=\mathrm{x}, \mathrm{y}, \mathrm{z}} \mathrm{C}_{\mathrm{ij}}^{\prime} \mathrm{S}_{\mathrm{i}}^{\prime} \overline{\mathrm{S}}_{\mathrm{j}}^{\prime} \equiv \mathrm{C}_{\mu \nu} \mathrm{S}^{\mu} \overline{\mathrm{S}}^{\nu}
\end{gathered}
$$

Explicit sums over $i$ and $j$ are denoted to indicate that all metric factors have been removed from the left hand side of eqn. A.9. Evaluating these new coefficients, taking care to include the appropriate factors from the metric matrix $\mathrm{g}_{\mu \nu}$, one gets:

$$
\begin{array}{ll}
\mathrm{C}_{\mathrm{x}}^{\prime}=-\mathrm{C}_{\mathrm{x}} & \overline{\mathrm{C}}_{\mathrm{x}}^{\prime}=-\overline{\mathrm{C}}_{\mathrm{x}} \\
\mathrm{C}_{\mathrm{y}}^{\prime}=-\mathrm{C}_{\mathrm{y}} & \overline{\mathrm{C}}_{\mathrm{y}}^{\prime}=-\overline{\mathrm{C}}_{\mathrm{y}} \\
\mathrm{C}_{\mathrm{z}}^{\prime}=-\gamma\left[\mathrm{C}_{\mathrm{z}}-\beta \mathrm{C}_{\mathrm{t}}\right] & \overline{\mathrm{C}}_{\mathrm{z}}^{\prime}=-\gamma\left[\overline{\mathrm{C}}_{\mathrm{z}}+\beta \overline{\mathrm{C}}_{\mathrm{t}}\right] \\
\mathrm{C}_{\mathrm{xx}}^{\prime}=\mathrm{C}_{\mathrm{xx}} & \mathrm{C}_{\mathrm{yx}}^{\prime}=\mathrm{C}_{\mathrm{yx}} \\
\mathrm{C}_{\mathrm{xy}}^{\prime}=\mathrm{C}_{\mathrm{xy}} & \mathrm{C}_{\mathrm{yy}}^{\prime}=\mathrm{C}_{\mathrm{yy}} \\
\mathrm{C}_{\mathrm{xz}}^{\prime}=\gamma\left[\mathrm{C}_{\mathrm{xz}}+\beta \mathrm{C}_{\mathrm{xt}}\right] & \mathrm{C}_{\mathrm{yz}}^{\prime}=\gamma\left[\mathrm{C}_{\mathrm{yz}}+\beta \mathrm{C}_{\mathrm{yt}}\right] \\
\mathrm{C}_{\mathrm{zx}}^{\prime}=\gamma\left[\mathrm{C}_{\mathrm{zx}}-\beta \mathrm{C}_{\mathrm{tx}}\right] & \mathrm{C}_{\mathrm{zy}}^{\prime}=\gamma\left[\mathrm{C}_{\mathrm{zy}}-\beta \mathrm{C}_{\mathrm{ty}}\right] \\
& \\
\mathrm{C}_{\mathrm{zz}}^{\prime}=\gamma^{2}\left[\mathrm{C}_{\mathrm{zz}}-\beta\left(\mathrm{C}_{\mathrm{tz}}-\mathrm{C}_{\mathrm{zt}}\right)-\beta^{2} \mathrm{C}_{\mathrm{tt}}\right]
\end{array}
$$


Explicitly, these become:

$$
\begin{aligned}
\mathrm{C}_{\mathrm{x}}^{\prime}=\overline{\mathrm{C}}_{\mathrm{x}}^{\prime}= & \mathrm{A}\left(\mathrm{s}-\mathrm{M}_{\mathrm{Z}}^{2}\right)\left(\frac{1}{\gamma}\right)\left\{2 \mathrm{a}_{\mathrm{f}} \mathrm{v}_{\mathrm{e}} \beta \cos \theta-\mathrm{v}_{\mathrm{f}}\right\} \\
& +\mathrm{B}\left\{\mathrm{v}_{\mathrm{e}} \mathrm{v}_{\mathrm{f}}^{2}-2 \mathrm{v}_{\mathrm{f}} \mathrm{a}_{\mathrm{f}}\left(\mathrm{v}_{\mathrm{e}}^{2}+\frac{1}{16}\right) \beta \cos \theta\right\} \frac{1}{\gamma} \sin \theta \\
\mathrm{C}_{\mathrm{y}}^{\prime}=\overline{\mathrm{C}}_{\mathrm{y}}^{\prime}= & \mathrm{AM} \mathrm{M}_{\mathrm{Z}} \Gamma_{\mathrm{Z}}\left(\frac{1}{2 \gamma}\right) \mathrm{a}_{\mathrm{f}} \beta \sin \theta \\
\mathrm{C}_{\mathrm{z}}^{\prime}=\overline{\mathrm{C}}_{\mathrm{z}}^{\prime}= & \mathrm{A}\left(\mathrm{s}-\mathrm{M}_{\mathrm{Z}}^{2}\right)\left\{2 \mathrm{a}_{\mathrm{f}} \mathrm{v}_{\mathrm{e}} \beta\left(1+\cos ^{2} \theta\right)-\mathrm{v}_{\mathrm{f}} \cos \theta\right\} \\
& +\mathrm{B}\left\{\mathrm{v}_{\mathrm{e}}\left(\mathrm{v}_{\mathrm{f}}^{\bar{z}}+\beta^{2} \mathrm{a}_{\mathrm{f}}^{2}\right) \cos \theta-2 \mathrm{v}_{\mathrm{f}} \mathrm{a}_{\mathrm{f}}\left(\mathrm{v}_{\mathrm{e}}^{2}+\frac{1}{16}\right) \beta\left(1+\cos ^{2} \theta\right)\right\}
\end{aligned}
$$

The diagonal terms of $\mathrm{C}_{\mathrm{ij}}^{\prime}$ become:

$$
\begin{aligned}
\mathrm{C}_{\mathrm{xx}}^{\prime}= & \mathrm{e}_{\mathrm{f}}^{2}\left\{\left(1+\frac{1}{\gamma^{2}}\right) \sin ^{2} \theta\right\} \\
& +\mathrm{A}\left(\mathrm{s}-\mathrm{M}_{\mathrm{Z}}^{2}\right)\left\{-2\left(1+\frac{1}{\gamma^{2}}\right) \mathrm{v}_{\mathrm{f}} \mathrm{v}_{\mathrm{e}} \sin ^{2} \theta\right\} \\
& +\mathrm{B}\left\{\left(\mathrm{v}_{\mathrm{f}}^{2}-\mathrm{a}_{\mathrm{f}}^{2}\right)+\frac{1}{\gamma^{2}}\left(\mathrm{v}_{\mathrm{f}}^{2}+\mathrm{a}_{\mathrm{f}}^{2}\right)\right\}\left(\mathrm{v}_{\mathrm{e}}^{2}+\frac{1}{16}\right) \sin ^{2} \theta \\
\mathrm{C}_{\mathrm{yy}}^{\prime}= & \mathrm{e}_{\mathrm{f}}^{2}\left\{-\beta^{2} \sin ^{2} \theta\right\} \\
& +\mathrm{A}\left(\mathrm{s}-\mathrm{M}_{\mathrm{Z}}^{2}\right) 2 \mathrm{v}_{\mathrm{f}} \mathrm{v}_{\mathrm{e}} \beta^{2} \sin ^{2} \theta \\
& +\mathrm{B}\left\{-\left(\mathrm{v}_{\mathrm{f}}^{2}-\mathrm{a}_{\mathrm{f}}^{2}\right)\left(\mathrm{v}_{\mathrm{e}}^{2}+\frac{1}{16}\right) \beta^{2} \sin ^{2} \theta\right\} \\
\mathrm{C}_{\mathrm{zz}}^{\prime}= & \mathrm{e}_{\mathrm{f}}^{2}\left\{1+\cos ^{2} \theta-\frac{\sin ^{2} \theta}{\gamma^{2}}\right\} \\
& +\mathrm{A}\left(\mathrm{s}-\mathrm{M}_{\mathrm{Z}}^{2}\right)\left\{\mathrm{a}_{\mathrm{f}} \beta \cos \theta-2 \mathrm{v}_{\mathrm{f}} \mathrm{v}_{\mathrm{e}}\left[\beta^{2}+\left(1+\frac{1}{\gamma^{2}}\right) \cos ^{2} \theta\right]\right\} \\
& +\mathrm{B}\left\{\left(\mathrm{v}_{\mathrm{e}}^{2}+\frac{1}{16}\right)\left[\left\{\left(\mathrm{v}_{\mathrm{f}}^{2}+\mathrm{a}_{\mathrm{f}}^{2}\right)+\frac{1}{\gamma^{2}}\left(\mathrm{v}_{\mathrm{f}}^{2}-\mathrm{a}_{\mathrm{f}}^{2}\right)\right\} \cos ^{2} \theta+\left(\mathrm{v}_{\mathrm{f}}^{2}+\mathrm{a}_{\mathrm{f}}^{2}\right) \beta^{2}\right]\right. \\
& \left.\quad \quad-2 \mathrm{v}_{\mathrm{f}} \mathrm{a}_{\mathrm{f}} \mathrm{v}_{\mathrm{e}} \beta \cos \theta\right\}
\end{aligned}
$$


The off-diagonal terms of $\mathrm{C}_{\mathrm{ij}}^{\prime}$ become:

$$
\begin{aligned}
\mathrm{C}_{\mathrm{xy}}^{\prime}=\mathrm{C}_{\mathrm{yx}}^{\prime}= & \mathrm{A}\left[2 \mathrm{M}_{\mathrm{z}} \Gamma_{\mathrm{z}} \mathrm{a}_{\mathrm{f}} \mathrm{v}_{\mathrm{c}} \beta \sin ^{2} \theta\right] \\
\mathrm{C}_{\mathrm{yz}}^{\prime}=\mathrm{C}_{\mathrm{zy}}^{\prime}= & \mathrm{A}\left[2 \mathrm{M}_{\mathrm{Z}} \Gamma_{\mathrm{z}}\left(\frac{1}{\gamma}\right) \mathrm{a}_{\mathrm{f}} \mathrm{v}_{\mathrm{e}} \beta \sin \theta \cos \theta\right] \\
\mathrm{C}_{\mathrm{xz}}^{\prime}=\mathrm{C}_{\mathrm{zx}}^{\prime}= & \mathrm{e}_{\mathrm{f}}^{2}\left(\frac{1}{\gamma}\right) \sin 2 \theta \\
& +\mathrm{A}\left(\mathrm{s}-\mathrm{M}_{\mathrm{Z}}^{2}\right)\left(\frac{1}{\gamma}\right)\left[\frac{1}{2} \mathrm{a}_{\mathrm{f}} \beta-4 \mathrm{v}_{\mathrm{f}} \mathrm{v}_{\mathrm{e}} \cos \theta\right] \sin \theta \\
- & +\mathrm{B}\left(\frac{1}{\gamma}\right)\left\{\mathrm{v}_{\mathrm{f}}^{2}\left(\mathrm{v}_{\mathrm{e}}^{2}+\frac{1}{16}\right) \sin 2 \theta-\mathrm{v}_{\mathrm{f}} \mathrm{a}_{\mathrm{f}} \mathrm{v}_{\mathrm{e}} \beta \sin \theta\right\}
\end{aligned}
$$

where

$$
\begin{aligned}
& \mathrm{A} \equiv \frac{\mathrm{e}_{\mathrm{f}}}{\sin ^{2} \theta_{\mathrm{W}} \cos ^{2} \theta_{\mathrm{W}}} \cdot \frac{\mathrm{s}}{\left(\mathrm{s}-\mathrm{M}_{\mathrm{Z}}^{2}\right)^{2}+\mathrm{M}_{\mathrm{Z}}^{2} \Gamma_{\mathrm{Z}}^{2}} \\
& \mathrm{~B} \equiv \frac{1}{\sin ^{4} \theta_{\mathrm{W}} \cos ^{4} \theta_{\mathrm{W}}} \cdot \frac{\mathrm{s}^{2}}{\left(\mathrm{~s}-\mathrm{M}_{\mathrm{Z}}^{2}\right)^{2}+\mathrm{M}_{\mathrm{Z}}^{2} \Gamma_{\mathrm{Z}}^{2}}
\end{aligned}
$$

\section{A.2 Event Generation Algorithm}

Before discussing the formulas used in simulating heavy lepton decay, it is useful to consider the general procedure for generating unweighted events. The sequence is as follows:

(1) Initial state radiation is produced according to the first line in eqn. 3.12 and according to eqn. 3.18 (the algorithm is contained in LUND code). The generated photon determines the Lorentz boost of the charged leptons in their own reducedenergy c.m. frame.

(2) Decay modes are chosen randomly and independently for the two leptons, based on the calculated total partial widths(to be discussed below).

(3) For each lepton, the energies of the decay products in the lepton rest frame are chosen randomly according to the appropriate spin-averaged differential partial widths.

(4) For each lepton, angles are chosen randomly and uniformly in phase space for the decay products of each lepton in that lepton's rest frame, ignoring spin-spin correlations for the moment. 
(5) The "polarimeter" vectors, $b$ and $\vec{b}$, defined by eqn. A.1, are calculated from the energies and directions of the decay products in each lepton rest frame. The polarimeter vectors determine the strength of the spin-spin correlations.

(6) The differcntial cross section weight

$$
\mathrm{W}_{\text {diff }} \equiv \frac{|\mathrm{F}|^{2}}{\mathrm{C}}
$$

is calculated and if $W_{\text {diff }}>r$, where $r$ is a random number in the range $[0,4]$, the event is accepted. Otherwise, the process begins again at step (4).

(7) -If any quarks are present, they must be "dressed" at this point into visible hadrons by the LUND program.

(8) All final state particles are boosted into the lab frame, and particles with finite lifetimes are allowed to decay and undergo the Mark II detector simulation.

\section{A.3 Decay Simulation}

In order to simulate lepton decay properly, one needs expressions for the differential partial decay widths for the various decay modes, including angular distributions dependent on the lepton's spin. Given below are the formulas used in determining the energy distributions of decay products, the polarimeter vectors, and the total partial widths, which determine the branching ratios.

\section{A.3.1 Fermionic decay (3-Body)}

The general expression for the decày of a heavy charged lepton into a neutral lepton and fermion-antifermion pair is given by ref. 72 :

$$
\begin{aligned}
d \Gamma\left(\mathrm{L}^{-} \rightarrow \nu_{\mathrm{L}} \overline{\mathrm{f}}_{1} \mathrm{f}_{2}\right)= & \frac{\mathrm{G}_{\mathrm{F}}^{2} \mathrm{M}_{\mathrm{L}}^{5}}{64 \pi^{5}} \frac{d \Omega_{\mathrm{f}_{2}} d \phi_{\overline{\mathrm{f}}_{1}-\mathrm{f}_{2}} d \mathrm{x}_{\mathrm{f}_{2}} d \mathrm{x}_{\overline{\mathrm{f}}_{1}}}{\left(1-\frac{\mathrm{M}_{\nu_{\mathrm{L}}}^{2}}{\mathrm{M}_{\mathrm{W}}^{2}}+\frac{\mathrm{M}_{\mathrm{L}}^{2}}{\mathrm{M}_{\mathrm{W}}^{2}}\left(1-\mathrm{x}_{\overline{\mathrm{f}}_{1}}-\mathrm{x}_{\mathrm{f}_{2}}\right)\right)^{2}+\frac{\Gamma_{\mathrm{W}}^{2}}{\mathrm{M}_{\mathrm{W}}^{2}}} \\
& \times\left(1+\mathrm{y}_{\overline{\mathrm{f}}_{1}}^{2}-\mathrm{y}_{\mathrm{f}_{2}}^{2}-\mathrm{y}_{\nu_{\mathrm{L}}}^{2}-\mathrm{x}_{\overline{\mathrm{f}}_{1}}\right)\left[\mathrm{x}_{\overline{\mathrm{f}}_{1}}-\frac{\mathrm{S}_{\mathrm{L}} \cdot \mathrm{p}_{\overline{\mathrm{f}}_{1}}}{\frac{1}{2} \mathrm{M}_{\mathrm{L}}}\right]
\end{aligned}
$$


where (V-A) coupling has been assumed and $\mathrm{W}$ propagator effects have been included. In this equation, $\mathrm{x}_{\mathrm{i}} \equiv 2 \mathrm{E}_{\mathrm{i}} / \mathrm{M}_{\mathrm{L}}$, and $\mathrm{y}_{\mathrm{i}} \equiv \mathrm{M}_{\mathrm{i}} / \mathrm{M}_{\mathrm{L}}$, while $\mathrm{M}_{\mathrm{W}}$ and $\Gamma_{\mathrm{W}}$ are the mass and total width of the $\mathrm{W}$ boson. In this analysis, the values $\mathrm{M}_{\mathrm{W}}=83 \mathrm{GeV}$ and $\Gamma_{\mathrm{W}}=3 \mathrm{GeV}$ are assumed. From the differential width, one can read off the polarimeter vector:

$$
\overrightarrow{\mathrm{b}}=\frac{\overrightarrow{\mathrm{p}}_{\overline{\mathrm{f}}_{1}}}{\frac{1}{2} \mathrm{M}_{\mathrm{L}} \mathrm{x}_{\overline{\mathrm{f}}_{1}}}=\frac{\overrightarrow{\mathrm{p}}_{\overline{\mathrm{f}}_{1}}}{\mathrm{E}_{\overline{\mathrm{f}}_{1}}}=\vec{\beta}_{\overline{\mathrm{f}}_{1}}
$$

Integrating the differential width over the available phase space with the assumption $\mathrm{M}_{\overline{\mathrm{f}}_{1}}=0$, one finds the following total partial width ${ }^{73}$ :

$$
\begin{aligned}
& \Gamma\left(L^{-} \rightarrow \nu_{L} \bar{f}_{1} f_{2}\right)=\frac{G_{F}^{2} M_{L}^{5}}{192 \pi^{3}}\left\{\frac{1}{2}\left[2-3 a^{3}-a^{2}+(5 b-14) a-13 b\right] \mathbf{r}\right. \\
& -\frac{3}{2}\left[a^{4}-2(b+2) a^{2}+b^{2}-4 b\right] L_{1} \\
& \left.+12 \mathrm{a} \sqrt{\mathrm{b}} \mathrm{L}_{2}\right\}
\end{aligned}
$$

where

$$
\begin{array}{rlrl}
\mathrm{a} & \equiv \mathrm{y}_{\mathrm{f}_{2}}^{2}+\mathrm{y}_{\nu_{\mathrm{L}}}^{2} & \mathrm{~L}_{1} \equiv \ln \left[\frac{\mathrm{M}_{\mathrm{L}}^{2}(1-\mathrm{a}+\mathrm{r})}{2 \mathrm{M}_{\mathrm{f}_{2}} \mathrm{M}_{\nu_{\mathrm{L}}}}\right] \\
\mathrm{b} \equiv\left(\mathrm{y}_{\mathrm{f}_{2}}^{2}-\mathrm{y}_{\nu_{\mathrm{L}}}^{2}\right)^{2} & \mathrm{~L}_{2} \equiv \ln \left[\frac{\mathrm{M}_{\mathrm{L}}^{2}\left(\mathrm{a}-\mathrm{b}-\mathrm{b}^{\frac{1}{2}} \mathrm{r}\right)}{2 \mathrm{M}_{\mathrm{f}_{2}} \mathrm{M}_{\nu_{\mathrm{L}}}}\right] \\
\mathrm{r} \equiv(1-2 \mathrm{a}+\mathrm{b})^{\frac{1}{2}} &
\end{array}
$$

For the decay $\mathrm{L}^{-} \rightarrow \nu_{\mathrm{L}} \mathrm{e}^{-} \bar{\nu}_{\mathrm{e}}$, both $\mathrm{M}_{\overline{\mathrm{f}}_{1}}$ and $\mathrm{M}_{\mathrm{f}_{2}}$ can be neglected, in which case eqn. A.11 reduces to

$$
\Gamma\left(\mathrm{L}^{-} \rightarrow \nu_{\mathrm{L}} \mathrm{e}^{-} \bar{\nu}_{\mathrm{e}}\right)=\frac{\mathrm{G}_{\mathrm{F}}^{2} \mathrm{M}_{\mathrm{L}}^{5}}{192 \pi^{3}}\left[1-8 \mathrm{y}_{\nu_{\mathrm{L}}}^{2}+8 \mathrm{y}_{\nu_{\mathrm{L}}}^{6}-\mathrm{y}_{\nu_{\mathrm{L}}}^{8}-24 \mathrm{y}_{\nu_{\mathrm{L}}}^{4} \ln \mathrm{y}_{\nu_{\mathrm{L}}}\right]
$$

In the case of $M_{\nu_{L}}, M_{\bar{f}_{1}}=0$, eqn. A.11 reduces to

$$
\Gamma\left(L^{-} \rightarrow \nu_{L} \bar{f}_{1} f_{2}\right)=\frac{G_{F}^{2} M_{L}^{5}}{192 \pi^{3}}\left[1-8 y_{f_{2}}^{2}+8 y_{f_{2}}^{6}-y_{f_{2}}^{8}-24 y_{f_{2}}^{4} \ln y_{f_{2}}\right]
$$

If all final state fermions have negligible masses, eqn. A.11 reduces to the familiar expression

$$
\Gamma\left(\mathrm{L}^{-} \rightarrow \nu_{\mathrm{L}} \mathrm{e}^{-} \bar{\nu}_{\mathrm{e}}\right)=\frac{\mathrm{G}_{\mathrm{F}}^{2} \mathrm{M}_{\mathrm{L}}^{5}}{192 \pi^{3}}
$$


The $\tau$ particle provides a check on these latter expressions. From eqn. A.13 and eqn. A.14, one expects

$$
\frac{\mathrm{B}\left(\tau^{-} \rightarrow \nu_{\tau} \mu^{-} \bar{\nu}_{\mu}\right)}{\mathrm{B}\left(\tau^{-} \rightarrow \nu_{\tau} \mathrm{e}^{-} \bar{\nu}_{\mathrm{e}}\right)}=0.97
$$

which is consistent with the measured value $e^{68} 1.01 \pm 0.04$.

Equation A.10 is used to generate decay product momenta for $\mathrm{L}^{-} \rightarrow \nu_{\mathrm{L}} \mathrm{e} \cdot \bar{\nu}_{\mathrm{e}}$, $\mathrm{L}^{-} \rightarrow \nu_{\mathrm{L}} \mu^{-} \bar{\nu}_{\mu}, \mathrm{L}^{-} \rightarrow \nu_{\mathrm{L}} \tau^{-} \bar{\nu}_{\tau}, \mathrm{L}^{-} \rightarrow \nu_{\mathrm{L}} \overline{\mathrm{u}} \mathrm{d}$, and $\mathrm{L}^{-} \rightarrow \nu_{\mathrm{L}} \overline{\mathrm{c} s}$, where for the last two, fragmentation into explicitly simulated decays is disabled. Total partial widths are calculated from eqn. A.12 for $\mathrm{L}^{-} \rightarrow \nu_{\mathrm{L}} \mathrm{e}^{-} \bar{\nu}_{\mathrm{e}}$ and from eqn. A.11 for $\mathrm{L}^{-} \rightarrow \nu_{\mathrm{L}} \mu^{-} \bar{\nu}_{\mu}$ and $\mathrm{L}^{-} \rightarrow \nu_{\mathrm{L}} \tau^{-} \bar{\nu}_{\tau}$, but the total partial widths for the quark decays require special care and will be discussed below.

\section{A.3.2 Scalar decay (2-Body)}

LULEPT simulates two scalar decay modes; $\mathrm{L}^{-} \rightarrow \nu_{\mathrm{L}} \pi^{-}$and $\mathrm{L}^{-} \rightarrow \nu_{\mathrm{L}} \mathrm{K}^{-}$, explicitly. Calculation of their partial widths is somewhat phenomenological and depends on other, experimentally measured decay rates. For example, the decays $\mathrm{L}^{-} \rightarrow \nu_{\mathrm{L}} \pi^{-}$ and $\pi^{-} \rightarrow \mu^{-} \nu_{\mu}$ both involve the difficult-to-calculate decay constant $f_{\pi}$, which describes the coupling strength at the $\pi-\mathrm{W}$ vertex. It is straightforward to show that

$$
\frac{d \Gamma\left(\mathrm{L}^{-} \rightarrow \nu_{\mathrm{L}} \pi^{-}\right)}{d \Omega}=\frac{\mathrm{G}_{\mathrm{F}}^{2} \mathrm{f}_{\pi}^{2} \cos ^{2} \theta_{\mathrm{c}}}{64 \pi^{2}} \mathrm{M}_{\mathrm{L}}^{3}\left(1-\mathrm{y}_{\pi}^{2}\right)^{2}\left(\Lambda_{\pi}+\mathrm{B}_{\pi} \mathrm{S} \cdot \hat{\mathrm{p}}_{\pi}\right)
$$

where $\hat{\mathrm{p}}_{\pi} \equiv \mathrm{p}_{\pi} / \mathrm{M}_{\mathrm{L}}$ in the $\mathrm{L}^{-}$rest frame, and

$$
\begin{aligned}
& \mathrm{A}_{\pi} \equiv\left[1-\frac{\mathrm{y}_{\nu_{\mathrm{L}}}^{2}}{\left(1+\mathrm{y}_{\pi}\right)^{2}}\right]^{\frac{1}{2}}\left[1-\frac{\mathrm{y}_{\nu_{\mathrm{L}}}^{2}}{\left(1-\mathrm{y}_{\pi}\right)^{2}}\right]^{\frac{1}{2}}\left[1-\frac{\mathrm{y}_{\nu_{\mathrm{L}}}^{2}\left(2+\mathrm{y}_{\pi}^{2}-\mathrm{y}_{\nu_{\mathrm{L}}}^{2}\right)}{1-\mathrm{y}_{\pi}^{2}}\right] \\
& \mathrm{B}_{\pi} \equiv\left[1-\frac{\mathrm{y}_{\nu_{\mathrm{L}}}^{2}}{\left(1+\mathrm{y}_{\pi}\right)^{2}}\right]\left[1-\frac{\mathrm{y}_{\nu_{\mathrm{L}}}^{2}}{\left(1-\mathrm{y}_{\pi}\right)^{2}}\right]\left[1-\mathrm{y}_{\nu_{\mathrm{L}}}^{2}\right]
\end{aligned}
$$

This equation applies also to $\mathrm{L}^{-} \rightarrow \nu_{\mathrm{L}} \mathrm{K}^{-}$with $\cos \theta_{\mathrm{c}} \rightarrow \sin \theta_{\mathrm{c}}$ and $\mathrm{y}_{\pi} \rightarrow \mathrm{yK}_{\mathrm{K}}$. The polarimeter vector can be read off from eqn. A.15:

$$
\overrightarrow{\mathrm{b}}=-\frac{\mathrm{B}_{\pi}}{\mathrm{A}_{\pi}} \hat{\mathrm{p}}_{\pi}
$$


Integrating over the solid angle of the pion, one gets

$$
\begin{aligned}
\Gamma\left(\mathrm{L}^{-}\right. & \left.\rightarrow \nu_{\mathrm{L}} \pi^{-}\right)=\frac{\mathrm{G}_{\mathrm{F}}^{2} \mathrm{f}_{\pi}^{2} \cos ^{2} \theta_{\mathrm{c}}}{16 \pi} \mathrm{M}_{\mathrm{L}}^{3}\left(1-\mathrm{y}_{\pi}^{2}\right)^{2} \\
& \times\left[1-\frac{\mathrm{y}_{\nu_{\mathrm{L}}}^{2}}{\left(1+\mathrm{y}_{\pi}\right)^{2}}\right]^{\frac{1}{2}}\left[1-\frac{\mathrm{y}_{\nu_{\mathrm{L}}}^{2}}{\left(1-\mathrm{y}_{\pi}\right)^{2}}\right]^{\frac{1}{2}}\left[1-\frac{\mathrm{y}_{\nu_{\mathrm{L}}}^{2}\left(2+\mathrm{y}_{\pi}^{2}-\mathrm{y}_{\nu_{\mathrm{L}}}^{2}\right)}{1-\mathrm{y}_{\pi}^{2}}\right]
\end{aligned}
$$

In the case $M_{\nu_{\mathbf{L}}}=0$, this reduces to

$$
\Gamma\left(\mathrm{L}^{-} \leftrightarrow \nu_{\mathrm{L}} \pi^{-}\right)=\frac{\mathrm{G}_{\mathrm{F}}^{2} \mathrm{f}_{\pi}^{2} \cos ^{2} \theta_{\mathrm{c}}}{16 \pi} \mathrm{M}_{\mathrm{L}}^{3}\left(1-\mathrm{y}_{\pi}^{2}\right)^{2}
$$

The value of $\mathrm{f}_{\pi}$ can be extracted from the branching ratio $\mathrm{B}\left(\pi^{-} \rightarrow \mu^{-} \nu_{\mu}\right)$, the lifetime of the pion, $\tau_{\pi}$, and the following expression for the total partial width of the decay $\pi^{-} \rightarrow \mu^{-} \nu_{\mu}$ :

$$
\Gamma\left(\pi^{-} \rightarrow \mu^{-} \nu_{\mu}\right)=\frac{\mathrm{G}_{\mathrm{F}}^{2} \mathrm{f}_{\pi}^{2} \cos ^{2} \theta_{\mathrm{c}}}{8 \pi \tau_{x}} \mathrm{M}_{\pi} \mathrm{M}_{\mu}^{2}\left[1-\left(\frac{\mathrm{M}_{\mu}}{\mathrm{M}_{\pi}}\right)^{2}\right]^{2}
$$

One expects for the $\tau$ :

$$
\frac{\mathrm{B}\left(\tau^{-} \rightarrow \nu_{\tau} \pi^{-}\right)}{\mathrm{B}\left(\tau^{-} \rightarrow \nu_{\tau} \mathrm{e}^{-} \bar{\nu}_{\mathrm{e}}\right)}=\frac{96 \pi^{3}}{\tau_{\pi}}\left(\frac{1-\left(\frac{\mathrm{M}_{\pi}}{\mathrm{M}_{\tau}}\right)^{2}}{1-\left(\frac{\mathrm{M}_{\mu}}{\mathrm{M}_{\pi}}\right)^{2}}\right)^{2} \frac{\mathrm{B}\left(\pi^{-} \rightarrow \mu^{-} \nu_{\mu}\right)}{\mathrm{G}_{\mathrm{F}}^{2} \mathrm{M}_{\tau}^{2} \mathrm{M}_{\mu}^{2} \mathrm{M}_{\pi}}=0.604
$$

which is consistent with the measured value $0.580 \pm 0.065$. The decay $\tau^{-} \rightarrow \nu_{\tau} \mathrm{K}^{-}$ can be treated the same way, with the substitutions described above. Using measured values of $\mathrm{B}\left(\mathrm{K}^{-} \rightarrow \mu^{-} \nu_{\mu}\right)$, and of the kaon lifetime, one predicts:

$$
\frac{\mathrm{B}\left(\tau^{-} \rightarrow \nu_{\tau} \mathrm{K}^{-}\right)}{\mathrm{B}\left(\tau^{-} \rightarrow \nu_{\tau} \mathrm{e}^{-} \bar{\nu}_{\mathrm{e}}\right)}=0.0395
$$

which, again, is consistent with the measured value $.0385 \pm 0.0098$.

\section{A.3.3 Vector decay (2-Body)}

A similar 2-body decay can occur involving a vector meson. LULEPT treats three such decays explicitly: $\mathrm{L}^{-} \rightarrow \nu_{\mathrm{L}} \rho^{-}, \mathrm{L}^{-} \rightarrow \nu_{\mathrm{L}} \mathrm{K}^{*^{-}}$, and $\mathrm{L}^{-} \rightarrow \nu_{\mathrm{L}} \mathrm{a}_{1}^{-}$. The Feynman 
diagram for the general process, $\mathrm{L}^{-} \rightarrow \nu_{\mathrm{L}} \mathrm{V}^{-}$where $\mathrm{V}^{-}$is a vector meson is shown in fig. A.2. The coupling at the V-W vertex cannot be calculated a priori, but like the decay constant $f_{\pi}$, it can be determined empirically from other processes. From the Conserved Vector Current(CVC) Theorem, ${ }^{74}$ the coupling is related through a weak isospin rotation to the electromagnetic coupling to $\mathrm{V}^{0}$. For example, the width for $\tau^{-} \rightarrow \nu_{\tau} \rho^{-}$can be expressed in terms of the cross section for $e^{+} e^{-} \rightarrow \rho^{0} \rightarrow \pi^{+} \pi^{-}$. A fit of this cross section yields the prediction: ${ }^{74}$

$$
\frac{\Gamma\left(\tau^{-} \rightarrow \nu_{\tau} \rho^{-}\right)}{\Gamma\left(\tau^{-} \rightarrow \nu_{\tau} \mathrm{e}^{-} \bar{\nu}_{\mathrm{e}}\right)}=1.30 \pm 0.10
$$

which is consistent with the measured value $1.25 \pm 0.12$. Such good agreement gives one confidence that the analytic calculation of $\Gamma\left(\mathrm{L}^{-} \rightarrow \nu_{L} \rho^{-}\right)$can be trusted, at least in form. Similar techniques lead to expressions for $\Gamma\left(\mathrm{L}^{-} \rightarrow \nu_{\mathbf{L}} \mathrm{K}^{*^{-}}\right)$and $\Gamma\left(\mathrm{L}^{-} \rightarrow\right.$ $\left.\nu_{L} a_{1}^{-}\right)$, with larger uncertainties.

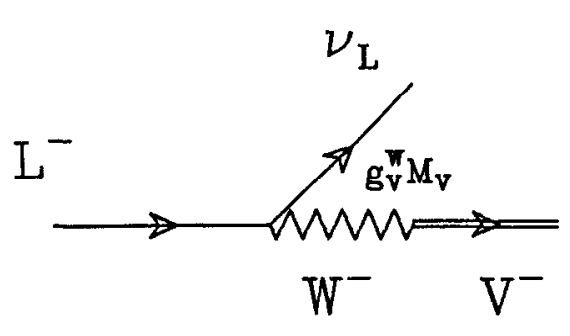

a)

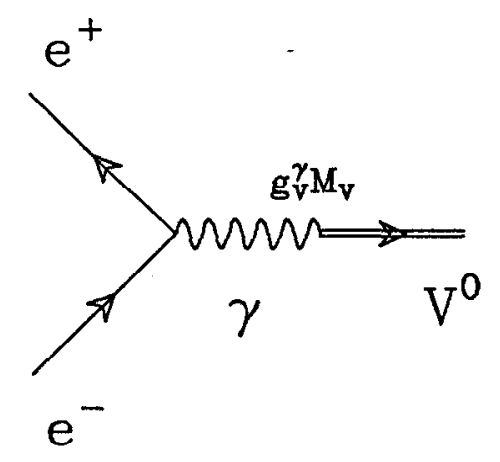

b)

Figure A.2. The CVC Theorem relates the coupling between the virtual $\mathrm{W}^{-}$and the $\mathrm{V}-$ in the decay $\mathrm{L}^{-} \rightarrow \nu_{\mathrm{L}} \mathrm{V}^{-}$to the coupling between the virtual $\gamma$ and the $\mathrm{V}^{0}$ in the process $\mathrm{e}^{+} \mathrm{e}^{-} \rightarrow \mathrm{V}^{0}$ through a weak isospin rotation.

The widths for these vector resonance decays have the same dependence on $M_{L}$, $M_{\nu_{L}}$, and on the appropriate values of $M_{v}$ and $\Gamma_{v}$. They differ, however, in their 
normalizations, which depend on measured coupling strengths. In principle, these normalizations can be found from processes such as $\mathrm{e}^{+} \mathrm{e}^{-} \rightarrow \rho^{0} \rightarrow \pi^{+} \pi^{-}$, as described above. But, as will be discussed below, LULEPT determines the normalizations factors by forcing agreement between ratios of observed branching fractions of the $\tau$ and the calculated values when $M_{L}$ is set to $M_{\tau}$ and $M_{\nu_{L}}$ set to zero.

This approach of forcing agreement with observation for the special case of $\mathrm{L}^{-} \equiv \tau^{-}$has the advantages of automatically producing the correct $\tau$ branching ratios when LULEPT is used to calculate conventional $\tau$ backgrounds, and of making the simulation slightly less sensitive to phenomenological assumptions.

.. In the narrow-width approximation, $\Gamma_{\mathrm{V}} \ll \mathrm{M}_{\mathrm{V}}$, the differential partial width for decay modes into vector mesons is

$$
d \Gamma\left(\mathrm{L}^{-} \rightarrow \nu_{\mathrm{L}} \mathrm{V}^{-}\right) \propto \mathrm{M}_{\mathrm{L}}^{3} \mathrm{M}_{\mathrm{v}}^{2}\left(1-\mathrm{y}_{\mathrm{v}}^{2}\right)^{2}\left(1+2 \mathrm{y}_{\mathrm{v}}^{2}\right)\left[\mathrm{A}_{\mathbf{v}}+\mathrm{B}_{\mathrm{v}}\left(\overrightarrow{\mathrm{S}}_{\mathrm{v}} \cdot \hat{\mathrm{p}}_{\mathrm{v}}\right)\right]
$$

where

$$
\begin{aligned}
& \mathrm{A}_{\mathrm{v}} \equiv\left[1-\frac{\mathrm{y}_{\nu_{\mathrm{L}}}^{2}}{\left(1+\mathrm{y}_{\mathrm{v}}\right)^{2}}\right]^{\frac{1}{2}}\left[1-\frac{\mathrm{y}_{\nu_{\mathrm{L}}}^{2}}{\left(1-\mathrm{y}_{\mathrm{v}}\right)^{2}}\right]^{\frac{1}{2}}\left[1-\frac{\mathrm{y}_{\nu_{\mathrm{L}}}^{2}\left(2-\mathrm{y}_{\mathrm{v}}^{2}-\mathrm{y}_{\nu_{\mathrm{L}}}^{2}\right)}{\left(1-\mathrm{y}_{\mathrm{v}}^{2}\right)^{2}\left(1+2 \mathrm{y}_{\mathrm{v}}^{2}\right)}\right] \\
& \mathrm{B}_{\mathrm{v}} \equiv \frac{1-2 \mathrm{y}_{\mathrm{v}}^{2}}{1+2 \mathrm{y}_{\mathrm{v}}^{2}}\left[1-\frac{\mathrm{y}_{\nu_{\mathrm{L}}}^{2}}{\left(1+\mathrm{y}_{\mathrm{v}}\right)^{2}}\right]\left[1-\frac{\mathrm{y}_{\nu_{\mathrm{L}}}^{2}}{\left(1-\mathrm{y}_{\mathrm{v}}\right)^{2}}\right]\left[1-\frac{\mathrm{y}_{\nu_{\mathrm{L}}}^{2}}{1-2 \mathrm{y}_{\mathrm{v}}^{2}}\right]
\end{aligned}
$$

and where $\hat{p}_{\mathbf{v}}$ is the unit vector in the direction of the $\mathrm{V}^{-}$in the $\mathrm{L}^{-}$rest frame. From eqn. A.18, the polarimeter vector can be read off:

$$
\vec{b}=\frac{B}{A} \hat{p}_{v}
$$

Integrating over the solid angle of $\mathrm{V}^{-}$gives the total partial width:

$$
\begin{aligned}
& \Gamma\left(\mathrm{L}^{-} \rightarrow \nu_{\mathrm{L}} \mathrm{V}^{-}\right) \propto \mathrm{M}_{\mathrm{L}}^{3} \mathrm{M}_{\mathrm{v}}^{2}\left(1-\mathrm{y}_{\mathrm{v}}^{2}\right)^{2}\left(1+2 \mathrm{y}_{\mathrm{v}}^{2}\right) \\
& \quad \times\left[1-\frac{\mathrm{y}_{\nu_{\mathrm{L}}}^{2}}{\left(1+\mathrm{y}_{\mathrm{v}}\right)^{2}}\right]^{\frac{1}{2}}\left[1-\frac{\mathrm{y}_{\nu_{\mathrm{L}}}^{2}}{\left(1-\mathrm{y}_{\mathrm{v}}\right)^{2}}\right]^{\frac{1}{2}}\left[1-\frac{\mathrm{y}_{\nu_{\mathrm{L}}}^{2}\left(2-\mathrm{y}_{\mathrm{v}}^{2}-\mathrm{y}_{\nu_{\mathrm{L}}}^{2}\right)}{\left(1-\mathrm{y}_{\mathrm{v}}^{2}\right)^{2}\left(1+2 \mathrm{y}_{\mathrm{v}}^{2}\right)}\right]
\end{aligned}
$$


In the case $M_{\nu_{L}}=0$, this reduces to

$$
\Gamma\left(\mathrm{L}^{-} \rightarrow \nu_{\mathrm{L}} \mathrm{V}^{-}\right) \propto \mathrm{M}_{\mathrm{L}}^{3} \mathrm{M}_{\mathrm{v}}^{2}\left(1-\mathrm{y}_{\mathrm{v}}^{2}\right)^{2}\left(1+2 \mathrm{y}_{\mathrm{v}}^{2}\right)
$$

For the modes $\mathrm{L}^{-} \rightarrow \nu_{\mathrm{L}} \mathrm{K}^{*^{-}}$and $\mathrm{L}^{-} \rightarrow \nu_{\mathrm{L}} \mathrm{a}_{1}^{-}$, the differential and total widths are calculated from eqn. A.18 and eqn. A.19. The decay $\mathrm{L}^{-} \rightarrow \nu_{\mathrm{L}} \rho^{-}$, however, warrants special treatment. The total width $\Gamma\left(\mathrm{L}^{-} \rightarrow \nu_{\mathrm{L}} \rho^{-}\right)$can be safely calculated from eqn. A.19, but as will be explained below, spin considerations demand a differential partial width that treats cxplicitly the final momenta of the $\pi^{-}$and the $\pi^{0}$.

\section{A.3.4 Vector decay (3-Body)}

When a spin $\frac{1}{2}$ lepton decays to a spin 1 particle and spin $\frac{1}{2}$ left-handed neutrino, only two of the three possible spin states are allowed for the vector particle. An extreme example illustrates this. Figure A.3 a shows a $\tau^{-}$with spin $+\frac{1}{2}$ decaying to a neutrino whose momentum is along the negative z-axis and to a $\rho^{-}$whose momentum is along the positive z-axis. Because the spin of the left-handed neutrino must be opposite to its direction of motion, its spin is $+\frac{1}{2}$. This requires the $\rho^{-}$to have spin $\mathrm{S}_{z}=0$. In fig. A.3 b, the $\tau^{-}$has spin $-\frac{1}{2}$. Again, the downward-going neutrino must have spin $+\frac{1}{2}$, but now the $\rho^{-}$must have spin -1 . In general, the $\rho^{-}$will not have a spin pointing in its own direction of motion in the $\tau^{-}$rest frame.

This constraint holds for any 2-body decay of the charged lepton into a vector meson and a left-handed neutrino, but its importance is greatest for vector particles that subsequently undergo 2-body decay, where spin effects will be strongest. Since the decay $\mathrm{L}^{-} \rightarrow \nu_{\mathrm{L}} \rho^{-}$involves the subsequent decay $\rho \rightarrow \pi^{-} \pi^{0}$ and since $\mathrm{B}\left(\mathrm{L}^{-} \rightarrow \nu_{\mathrm{L}} \rho^{-}\right)$can be substantial $(\approx 22 \%$ for the $\tau)$, the decay mode is treated differently from $\mathrm{L}^{-} \rightarrow \nu_{\mathrm{L}} \mathrm{K}^{*^{-}}$and $\mathrm{L}^{-} \rightarrow \nu_{\mathrm{L}} \mathrm{a}_{1}^{-}$.

In the approximation that $\mathrm{M}_{\pi^{-}} \approx \mathrm{M}_{\pi^{0}}$, the differential partial width can be written: ${ }^{75}$

$$
d \Gamma\left(\mathrm{L}^{-} \rightarrow \nu_{\mathrm{L}} \rho^{-}\right) \propto \frac{\left|\mathrm{F}_{\pi}\right|^{2}}{\mathrm{M}_{\mathrm{L}}}\left[\mathrm{A}_{\rho}+\mathrm{B}_{\rho_{\mu}} \mathrm{S}^{\mu}\right] d \mathrm{Lips}
$$




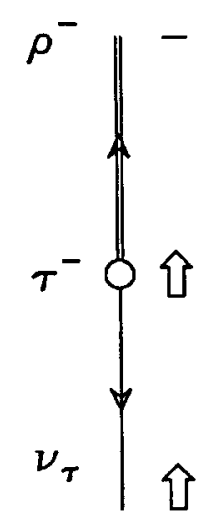

$-$

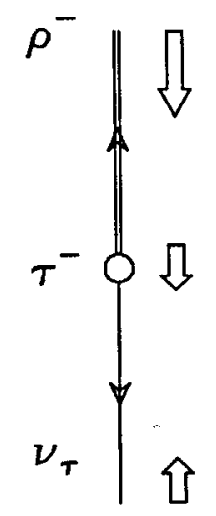

b)

Figure A.3. Quantizing angular momentum along the direction of the $\rho^{-}$in $\tau^{-} \rightarrow \nu_{\tau} \rho^{-}$reveals that the $\rho^{-}$can end up in only two of its three possible spin states.

where

$$
\begin{aligned}
\mathrm{A}_{\rho} & \equiv 2\left(\mathrm{p}_{\mathrm{L}} \cdot \mathrm{q}\right)\left(\mathrm{p}_{\nu_{\mathrm{L}}} \cdot \mathrm{q}\right)-\left(\mathrm{p}_{\mathrm{L}} \cdot \mathrm{p}_{\nu_{\mathrm{L}}}\right) \mathrm{M}_{\pi \pi}^{2} \\
\mathrm{~B}_{\rho}{ }^{\mu} & \equiv-\left[2 \mathrm{M}_{\mathrm{L}}\left(\mathrm{p}_{\nu_{\mathrm{L}}} \cdot \mathrm{q}\right) \mathrm{q}^{\mu}-\mathrm{M}_{\mathrm{L}} \mathrm{q}^{2} \mathrm{p}_{\nu_{\mathrm{L}}}^{\mu}\right] \\
\mathrm{q} & \equiv \mathrm{p}_{\pi^{-}}-\mathrm{p}_{\pi^{0}}
\end{aligned}
$$

and where $\left|F_{\pi}\right|^{2}$ depends on $M_{\pi \pi}^{2}$, the invariant mass squared of the two pions together, and is defined empirically by

$$
\sigma\left(\mathrm{e}^{+} \mathrm{e}^{-} \rightarrow \pi^{+} \pi^{-}\right)=\frac{\pi}{3} \frac{\alpha^{2}}{\mathrm{~s}}\left(1-\frac{4 \mathrm{M}_{\pi}^{2}}{\mathrm{~s}}\right)^{\frac{3}{2}}\left|\mathrm{~F}_{\pi}\right|^{2}
$$

To a good approximation,

$$
\left|\mathrm{F}_{\pi}\right|^{2} \propto \frac{1}{\left(\mathrm{M}_{\pi \pi}^{2}-\mathrm{M}_{\rho}^{2}\right)^{2}+\mathrm{M}_{\rho}^{2} \Gamma_{\rho}^{2}}
$$

The differential Lorentz invariant phase space volume element can be written

$$
d \text { Lips }=\frac{1}{2^{11} \pi^{5}} d \Omega_{\nu_{\mathrm{L}}} \frac{\lambda^{\frac{1}{2}}\left(\mathrm{M}_{\mathrm{L}}^{2}, \mathrm{M}_{\nu_{\mathrm{L}}}^{2}, \mathrm{M}_{\pi \pi}^{2}\right)}{\mathrm{M}_{\mathrm{L}}^{2}} d \mathrm{M}_{\pi \pi}^{2} d \Omega_{\pi^{0}}^{\prime} \frac{\lambda^{\frac{1}{2}}\left(\mathrm{M}_{\pi \pi}^{2}, \mathrm{M}_{\pi}^{2}, \mathrm{M}_{\pi}^{2}\right)}{\mathrm{M}_{\pi \pi}^{2}}
$$

where

$$
\lambda(x, y, z) \equiv x^{2}+y^{2}+z^{2}-2(x y+y z+x z)
$$


$d \Omega_{\nu_{\mathrm{L}}}$ is the solid angle element of the neutrino in the $\mathrm{L}^{-}$rest frame, and $d \Omega_{\pi^{0}}^{\prime}$ is the solid angle element of the $\pi^{0}$ in the $\pi^{-}-\pi^{0}$ rest frame. The polarimeter vector is easily read off:

$$
\overrightarrow{\mathrm{b}}=-\frac{\overrightarrow{\mathrm{B}}_{\rho}}{\mathrm{A}_{\rho}}
$$

\section{A.3.5 Quark decay (total partial width)}

Once a quark decay mode has been chosen, it is straightforward to determine the momenta of the quarks and of the neutrino from eqn. A.10 and then to fragment the quarks with the LUND program. What is not straightforward is to determine how often- to generate the quark decay modes, that is, to determine a reasonable total partial width.

The scheme used in LULEPT is to assume that the process $\mathrm{L}^{-} \rightarrow \nu_{\mathrm{L}}$ (hadrons) ${ }^{-}$ for $\mathrm{M}_{\text {(hadrons) }}>\mathrm{M}_{\mathrm{a}_{1}}$ is dominated by production of a virtual vector particle which materializes as visible hadrons. Invoking CVC as in the decay $\mathrm{L}^{-} \rightarrow \nu_{\mathrm{L}} \rho^{-}$considered above, one further assumes that the decay width for a particular value of $M_{\text {(hadrons) }}$ is proportional to the $\mathrm{e}^{+} \mathrm{e}^{-}$annihilation cross section into hadrons at $\mathrm{s}=\mathrm{M}_{\text {(hadrons) }}^{2}$.

Neglecting kinematic suppression near thresholds, one can approximate the total hadronic annihilation cross section by

$$
\sigma\left(\mathrm{e}^{+} \mathrm{e}^{-} \rightarrow \text { hadrons }\right)=\frac{4 \pi}{3} \frac{\alpha^{2}}{\mathrm{~s}} \mathrm{R}(\mathrm{s})
$$

where $R(s)$ depends on the sum of the squares of quark electric charges for those quarks light enough to be produced:

$$
R(s)=3 \sum_{i} Q_{i}^{2} \quad(i=u d s c b)
$$

for all $\mathrm{i}$ such that $4 \mathrm{M}_{\mathrm{i}}^{2}<\mathrm{s}$. The factor of 3 is present because the final state quarks can end up in any of three color singlet states. Just above strange quark threshold, $R=2$; above charm threshold, $R=3 \frac{1}{3}$; and above bottom threshold, $R=3 \frac{2}{3}$. 
Roughly, the prescription used by LULEPT to calculate the total non-resonant hadronic decay width is

$$
\left.\Gamma\left(\mathrm{L}^{-} \rightarrow \nu_{\mathrm{L}} \text { (hadrons) }\right)^{-}\right)=\mathrm{N}_{\text {tot }} \int_{\Lambda^{2}}^{\left(\mathrm{M}_{\mathrm{L}}-\mathrm{M}_{\nu_{\mathrm{L}}}\right)^{2}} \frac{\mathrm{R}(\mathrm{s})}{\mathrm{s}} \Gamma_{\mathrm{L}_{-\rightarrow \nu_{\mathrm{L}}} \mathrm{v}^{-}}(\mathrm{s}) d \mathrm{~s}
$$

where $\Gamma_{\mathrm{L}^{-} \rightarrow v_{\mathrm{L}} \mathrm{v}^{-}}(\mathrm{s})$ is calculated from eqn. A.19 with $\mathrm{M}_{\mathrm{v}}^{2}$ set equal to s. $\Lambda$ is approximately the mass of the $a_{1}$, and $N_{\text {tot }}$ is determined by the requirement that as $\mathrm{M}_{\mathrm{L}} \rightarrow \infty$, the hadronic partial width for ud production be three times the partial width for e- $\bar{\nu}_{\mathrm{e}}$ production. This constraint on $\mathrm{N}_{\text {tot }}$ is necessary because in the high "energy limit, one expects each of the four processes in fig. A.4 to have identical widths, as the exact nature of the quark fragmentation becomes unimportant. The precise value of $\Lambda$ is chosen so that the value of the integral gives a partial width consistent with the partial width of the corresponding measured decay modes of the $\tau$ when $\mathrm{M}_{\mathrm{L}}=1.784 \mathrm{GeV}$ and $\mathrm{M}_{\nu_{\mathrm{L}}}=0$. In other words, one requires

$$
\begin{aligned}
& \frac{\left.\Gamma\left(\tau^{-} \rightarrow \nu_{\tau} \text { (hadrons }\right)^{-}\right)}{\left.\Gamma\left(\tau^{-} \rightarrow \nu_{\tau} \text { (hadrons }\right)^{-}\right)+\sum_{\mathrm{i}} \Gamma\left(\tau^{-} \rightarrow \nu_{\tau}(\text { exclusivemode })_{\mathrm{i}}\right)} \\
& \quad=1-\sum_{\mathrm{i}} \mathrm{B}\left(\tau^{-} \rightarrow \nu_{\tau}(\text { (exclusive mode })_{\mathrm{i}}\right)
\end{aligned}
$$

where

$$
\begin{aligned}
\sum_{\mathrm{i}} \Gamma\left(\tau^{-} \rightarrow \nu_{\tau}(\text { exclusive mode })_{\mathrm{i}}\right)= & \Gamma\left(\tau^{-} \rightarrow \nu_{\tau} \mathrm{e}^{-} \bar{\nu}_{\mathrm{e}}\right)+\Gamma\left(\tau^{-} \rightarrow \nu_{\tau} \mu^{-} \bar{\nu}_{\mu}\right) \\
& +\Gamma\left(\tau^{-} \rightarrow \nu_{\tau} \pi^{-}\right)+\Gamma\left(\tau^{-} \rightarrow \nu_{\tau} \mathrm{K}^{-}\right) \\
& +\Gamma\left(\tau^{-} \rightarrow \nu_{\tau} \mathrm{a}_{1}^{-}\right)+\Gamma\left(\tau^{-} \rightarrow \nu_{\tau} \mathrm{K}^{*^{-}}\right) \\
& +\Gamma\left(\tau^{-} \rightarrow \nu_{\tau} \rho^{-}\right)
\end{aligned}
$$

The purely leptonic decays $\Gamma\left(\tau^{-} \rightarrow \nu_{\tau} \mathrm{e}^{-} \bar{\nu}_{\mathrm{e}}\right)$ and $\Gamma\left(\tau^{-} \rightarrow \nu_{\tau} \mu^{-} \bar{\nu}_{\mu}\right)$ are calculated exactly, and the remaining exclusive partial widths are constrained to obey

$$
\frac{\Gamma\left(\tau^{-} \rightarrow \nu_{\tau}(\text { exclusivemode })_{\mathrm{i}}\right)}{\Gamma\left(\tau^{-} \rightarrow \nu_{\tau} \mathrm{e}^{-} \bar{\nu}_{\mathrm{e}}\right)}=\frac{\mathrm{B}\left(\tau^{-} \rightarrow \nu_{\tau}(\text { exclusive mode })_{\mathrm{i}}\right)}{\mathrm{B}\left(\tau^{-} \rightarrow \nu_{\tau} \mathrm{e}^{-} \bar{\nu}_{\mathrm{e}}\right)}
$$


a)

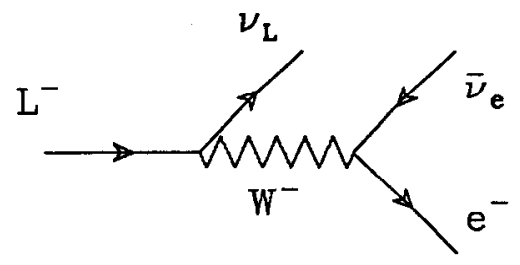

b)

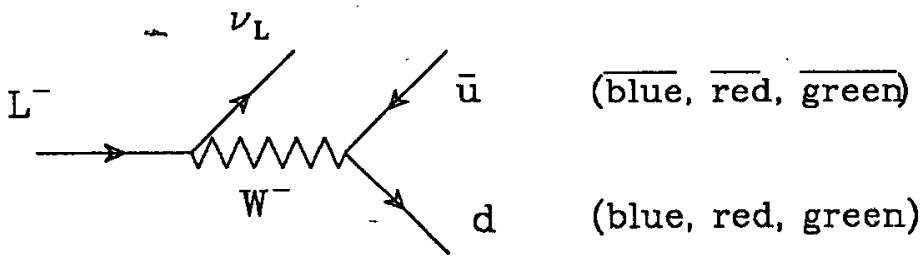

Figure A.4. In the limit $M_{L} \rightarrow-\infty$, the partial decay width for $\mathrm{L}^{-} \rightarrow \nu_{\mathbf{L}}$ (hadrons) ${ }^{-}$should be three times the partial width for $\mathrm{L}^{-} \rightarrow \nu_{\mathrm{L}} \mathrm{e}^{-} \bar{\nu}_{\mathrm{e}}$.

where the branching ratios are the experimentally measured values.

The total continuum hadronic width can be rewritten as*

$$
\begin{aligned}
\Gamma\left(\mathrm{L}^{-}\right. & \left.\left.\rightarrow \nu_{\mathrm{L}} \text { (hadrons }\right)^{-}\right)=\sum_{\mathrm{i}=1}^{2} 3\left(\frac{\mathrm{G}_{\mathrm{F}}^{2} \mathrm{M}_{\mathrm{L}}^{5}}{192 \pi^{3}}\right) \int_{\Lambda_{\mathrm{i}}^{2} / \mathrm{M}_{\mathrm{L}}^{2}}^{\left(\mathrm{M}_{\mathrm{L}}-\mathrm{M}_{\nu_{\mathrm{L}}}\right) / \mathrm{M}_{\mathrm{L}}^{2}} d \mathrm{y}_{\mathrm{v}}^{2} \\
& \times\left[\left(1-\mathrm{y}_{\mathrm{v}}^{2}\right)^{2}-2 \mathrm{y}_{\nu_{\mathrm{L}}}^{2}\left(1+\mathrm{y}_{\mathrm{v}}^{2}\right)+\mathrm{y}_{\nu_{\mathrm{L}}}^{4}\right]^{\frac{1}{2}}\left[1+\mathrm{y}_{\mathrm{v}}^{2}-2 \mathrm{y}_{\mathrm{v}}^{4}-2 \mathrm{y}_{\nu_{\mathrm{L}}}^{2}+\mathrm{y}_{\mathrm{v}}^{2} \mathrm{y}_{\nu_{\mathrm{L}}}^{2}+\mathrm{y}_{\nu_{\mathrm{L}}}^{4}\right]
\end{aligned}
$$

for each $\Lambda_{\mathrm{i}}<\mathrm{M}_{\mathrm{L}}-\mathrm{M}_{\nu_{\mathrm{L}}}$, where $\Lambda_{1}$ corresponds to the continuum cutoff(=1.0 GeV) and $\Lambda_{2}$ corresponds to the $\bar{c}$ s threshold $(=2.0 \mathrm{GeV})$. Normalization has been fixed so that as $\mathrm{M}_{\mathrm{L}} \rightarrow \infty, \Gamma\left(\mathrm{L}^{-} \rightarrow \nu_{\mathrm{L}} \overrightarrow{\mathrm{u}} \mathrm{d}\right) \rightarrow 3 \Gamma\left(\mathrm{L}^{-} \rightarrow \nu_{\mathrm{L}} \mathrm{e}^{-} \vec{\nu}_{\mathrm{e}}\right)$. Note that $\Lambda_{1}$ does not

* Because quark pair production through a charged W involves mass thresholds different from those appearing in production through a neutral photon, the simple prescription of substituting $R(s)$ into the integral is not really correct. In this calculation, Cabibbo mixing is neglected; that is, only $\overline{\mathrm{u} d}$ and $\overline{\mathrm{cs}}$ are considered. This approach is reasonable, since the $\bar{u}$ s threshold is well below $\Lambda_{1}$, and the correction due to $\overline{\mathrm{c}} \mathrm{d}$ production threshold being slightly below the $\overline{\mathrm{cs}}$ threshold will be small. These approximations, however, apply only to the total width calculation. The LUND program correctly simulates appropriate threshold and mixing effects once LULEPT has chosen to perform a hadronic continuum decay. 
correspond to the actual minimum $\mathrm{Q}\left(\mathrm{Q}_{\min }=1.275 \mathrm{GeV}\right)$ of continuum production simulated in LULEPT. It serves more as a free parameter to duplicate the correct $\tau$ branching ratio in a necessarily flawed method of hadronic decay simulation. The closeness of $\Lambda_{1}$ to $Q_{\min }$ is one measure of the validity of this scheme.

The integral can be evaluated analytically to give

$$
\begin{aligned}
\Gamma\left(\mathrm{L}^{-}\right. & \left.\left.\rightarrow \nu_{\mathrm{L}} \text { (hadrons }\right)^{-}\right)=\frac{\mathrm{G}_{\mathrm{F}}^{2} \mathrm{M}_{\mathrm{L}}^{5}}{8 \pi^{3}} \mathrm{y}_{\nu_{\mathrm{L}}}^{2} \\
& \times\left\{\mathrm{y}_{\nu_{\mathrm{L}}}^{2}\left[-\frac{2 \sin \theta_{\mathrm{i}}}{\cos ^{4} \theta_{\mathrm{i}}}-\frac{\sin \theta_{\mathrm{i}}}{\cos ^{2} \theta_{\mathrm{i}}}+3 \ln \left(\sec \theta_{\mathrm{i}}+\tan \theta_{\mathrm{i}}\right)\right]+2 \mathrm{y}_{\nu_{\mathrm{L}}}\left(1+\mathrm{y}_{\nu_{\mathrm{L}}}^{2}\right) \tan ^{3} \theta_{\mathrm{i}}\right\}
\end{aligned}
$$

where

$$
\cos \theta_{\mathrm{i}} \equiv \frac{2 \mathrm{y}_{\nu_{\mathrm{L}}}}{1+\mathrm{y}_{\nu_{\mathrm{L}}}^{2}-\mathrm{y}_{\Lambda_{\mathrm{i}}}^{2}} \quad \mathrm{y}_{\Lambda_{\mathrm{i}}} \equiv \frac{\Lambda_{\mathrm{i}}}{\mathrm{M}_{\mathrm{L}}}
$$

\section{A.3.6 Branching ratios}

As already discussed, total partial width calculations are normalized so as to agree with measured $\tau$ branching ratios when $\mathrm{M}_{\mathrm{L}}=\mathrm{M}_{\tau}$ and $-\mathrm{M}_{\nu_{\mathrm{L}}}=0$. A problem with this approach immediately presents itself. The measured inclusive branching ratio for 1-prong decays of the $\tau$ does not agree with the sum of measured exclusive 1-prong branching ratios. ${ }^{59}$ In other words, there appear to be "missing" decay modes, which make up $7 \pm 2 \%$ of the tau's total width. ${ }^{41}$ Despite much recent effort, ${ }^{41,76}$ the discrepancy remains unresolved.

Since LULEPT cannot simulate unknown missing decay modes, the discrepancy must be addressed. The approach chosen is to remove the discrepancy in the simulation by renormalizing exclusive $\tau$ branching ratios so that they sum to unity. A recent analysis of Mark II data, ${ }^{77}$ in which a simultaneous fit was performed on particle distributions from tagged $\tau$ decays, determined the most likely values for the known exclusive branching ratios. In that analysis, the tagging constrained the measured branching ratios to sum to unity. The branching ratios obtained from that measurement led unsurprisingly to values higher than world average for most decay 
modes, with the bulk of the original discrepancy ending up in the rho mode and in the 1-prong modes with more than one neutral pion.

LULEPT chooses branching ratios compatible with the Mark II measurement according to the following prescription. The widths for $\tau^{-} \rightarrow \nu_{\tau} \mathrm{e}^{-} \bar{\nu}_{\mathrm{e}}, \tau^{-} \rightarrow \nu_{\tau} \mu^{-} \bar{\nu}_{\mu}$, $\tau^{-} \rightarrow \nu_{\tau} \pi^{-}$, and $\tau^{-} \rightarrow \nu_{\tau} \mathrm{K}^{-}$are calculated exactly, as described above, where the lifetimes and appropriate branching ratios of charged pions and kaons are used. The ratio of $\mathrm{B}\left(\tau^{-} \rightarrow \nu_{\tau} \rho^{-}\right)$divided by $\mathrm{B}\left(\tau^{-} \rightarrow \nu_{\tau} \mathrm{e}^{-} \bar{\nu}_{\mathrm{e}}\right)$ is forced to agree with the Mark II value for the same ratio. The branching ratio for $\tau^{-} \rightarrow \nu_{\tau} \mathrm{K}^{*^{-}}$is chosen to be $\mathrm{B}\left(\tau^{-} \rightarrow \nu_{\tau} \rho^{-}\right)$times $\tan ^{2} \theta_{c}$. Since the $\mathrm{a}_{1}^{-}$is expected to decay predominantly to $\pi^{-} \pi^{+} \pi^{-}$and to $\pi^{-} \pi^{0} \pi^{0}$ in equal proportions, the branching ratio for $\mathrm{L}^{-} \rightarrow \nu_{\mathrm{L}} \mathrm{a}_{1}^{-}$ is chosen to be twice the Mark II value for $\mathrm{B}\left(\tau^{-} \rightarrow \nu_{\tau} \pi^{-} \pi^{+} \pi^{-}\right)$. The value for $\mathrm{B}\left(\tau^{-} \rightarrow \nu_{\tau} \pi^{-} \pi^{+} \pi^{-}\right)$is more reliable than the value for $\mathrm{B}\left(\tau^{-} \rightarrow \nu_{\tau} \pi^{-} \pi^{0} \pi^{0}\right)$ because of the difficulty in reconstructing multiphoton events. The branching ratio for $\mathrm{L}^{-} \rightarrow \nu_{\mathrm{L}} \overline{\mathrm{u}} \mathrm{d}$ is then chosen according to the algorithm described in the previous section. Once the branching ratios for the $\tau$ decay modes have been fixed, the branching ratios for arbitrary $\mathrm{M}_{\mathrm{L}}$ and $\mathrm{M}_{\nu_{\mathrm{L}}}$ are determined, except for $\mathrm{B}\left(\mathrm{L}^{-} \rightarrow \nu_{\mathrm{L}} \overline{\mathrm{s}} \mathrm{s}\right)$, which was discussed earlier. The partial width for $\mathrm{L}^{-} \rightarrow \nu_{\mathrm{L}} \tau^{-} \bar{\nu}_{\tau}$, when kinematically allowed, is calculated exactly, as for $\mathrm{L}^{-} \rightarrow \nu_{\mathrm{L}} \mathrm{e}^{-} \bar{\nu}_{\mathrm{e}}$ and $\mathrm{L}^{-} \rightarrow \nu_{\mathrm{L}} \mu^{-} \bar{\nu}_{\mu}$. Table A.1 shows the $\tau$ branching ratios assumed by LULEPT.

The fact that the missing decay modes have not been properly simulated requires that a correction be applied to any efficiencies calculated from Montc Carlo studics. Specifically, if a simulated event in which one lepton decays through mode $i$ and the other through mode $\mathrm{j}$ passes all selection cuts, then that event is weighted according to an efficiency correction $f_{i} \cdot f_{j}$ where

$$
\mathrm{f}_{\mathrm{i}} \equiv \frac{\mathrm{B}\left(\tau^{-} \rightarrow \nu_{\tau}(\text { mode })_{\mathrm{i}}\right)(\text { calculated })}{\mathrm{B}\left(\tau^{-} \rightarrow \nu_{\tau}(\text { mode })_{\mathrm{i}}\right)(\text { worldaverage })}
$$

World average values for $\tau$ branching ratios are taken from a review by Hitlin. ${ }^{78 *}$ The

* Reference 78 includes average values of $\mathrm{B}\left(\tau^{-} \rightarrow \nu_{\tau} \mathrm{e}^{-} \bar{\nu}_{\mathrm{e}}\right)$ and $\mathrm{B}\left(\tau^{-} \rightarrow \nu_{\tau} \mu^{-} \bar{\nu}_{\mu}\right)$ 
Table A.1. The following $\tau$ branching ratios are used to normalize the partial width calculations of LULEPT. Values are based on ref. 77 and on analytic calculations.

\begin{tabular}{|c|c|}
\hline Decay & B.R.(\%) \\
\hline$\tau^{-} \rightarrow \nu_{\tau} \mathrm{e}^{-} \bar{\nu}_{\mathrm{e}}$ & 19.1 \\
\hline$\tau^{-} \rightarrow \nu_{\tau} \mu^{-} \bar{\nu}_{\bar{\mu}}$ & 18.6 \\
\hline$\tau^{-} \rightarrow \nu_{\tau} \pi^{-}$ & 11.4 \\
\hline$\tau^{-} \rightarrow \nu_{\tau} \rho^{-}$ & 25.6 \\
\hline$\tau^{-} \rightarrow \nu_{\tau} \mathrm{K}^{-}$ & 0.7 \\
\hline$\tau^{-} \rightarrow \nu_{\tau} \mathrm{K}^{*^{-}}$ & 1.3 \\
\hline$\tau^{-} \rightarrow \nu_{\tau} \mathrm{a}_{1}^{-}$ & 13.0 \\
\hline$\tau^{-} \rightarrow \nu_{\tau} \overline{\mathrm{ud}}$ & 10.2 \\
\hline
\end{tabular}

two decay modes inaccessible to the $\tau$ lepton, $\mathrm{L}^{-} \rightarrow \nu_{\mathrm{L}} \overline{\mathrm{cS}}$ and $\mathrm{L}^{-} \rightarrow \nu_{\mathrm{L}} \tau^{-} \bar{\nu}_{\tau}$, are assigned an $\mathrm{f}_{\mathrm{i}}$ value equal to that for the mode $\mathrm{L}^{-} \rightarrow \nu_{\mathrm{L}} \mathrm{e}^{-} \vec{\nu}_{\mathrm{e}}$. Table A.2 gives the efficiency corrections applied to the simulated decay modes.

It is useful to examine the dependence of the calculated branching ratios on the values of $\mathrm{M}_{\mathrm{L}}$ and $\mathrm{M}_{\nu_{\mathrm{L}}}$. Plotted in fig. A.5 are calculated branching ratios as functions of the mass difference between the charged and neutral lepton for three values of the charged lepton's mass. These figures indicate that the branching ratios are far more sensitive to the mass difference than to the value of $M_{L}$, and that for $\delta \mathrm{M}>5 \mathrm{GeV}$, the width is dominated by the leptonic and hadronic continuum decay modes, whose branching ratios are insensitive to both $\mathrm{M}_{\mathrm{L}}$ and $\delta \mathrm{M}$. The curves shown begin at $\delta \mathrm{M}=250 \mathrm{MeV} / \mathrm{c}^{2}$ because of extremely rapid variations near the charged

where e- $\mu$ universality is imposed. Since the same imposition is made in LULEPT, these are the values used. The world average value for $\mathrm{B}\left(\tau^{-} \rightarrow \nu_{\tau} \mathrm{a}_{1}^{-}\right)$is chosen to be twice the average value for $\mathrm{B}\left(\tau^{-} \rightarrow \nu_{\tau} \pi^{-} \pi^{+} \pi^{-}\right)$. The average value for $\mathrm{B}\left(\tau^{-} \rightarrow \nu_{\tau} \overline{\mathrm{u}} \mathrm{d}\right)$ is determined as above by summing all exclusive modes not explicitly simulated by LULEPT. 
Table A.2. Efficiency corrections for LULEPT decay modes. Because of missing decay modes which cannot be simulated, branching ratios calculated by LULEPT are in general higher than the world average values, requiring a weighting correction be applied to LULEPT events passing selection cuts. The event weight correction is the product of the two decay mode corrections appropriate for the simulated event.

\begin{tabular}{|l|c|}
\hline \multicolumn{1}{|c|}{ Decay } & Correction \\
\hline $\mathrm{L}^{-} \rightarrow \nu_{\mathrm{L}} \mathrm{e}^{-} \bar{\nu}_{\mathrm{e}}$ & 0.96 \\
\hline $\mathrm{L}^{-} \rightarrow \nu_{\mathrm{L}} \mu^{-} \bar{\nu}_{\mu}$ & 0.95 \\
\hline $\mathrm{L}^{-} \rightarrow \nu_{\mathrm{L}} \pi^{-}$ & 0.95 \\
\hline $\mathrm{L}^{-} \rightarrow \nu_{\mathrm{L}} \rho^{-}$ & 0.87 \\
\hline $\mathrm{L}^{-} \rightarrow \nu_{\mathrm{L}} \mathrm{K}^{-}$ & 0.97 \\
\hline $\mathrm{L}^{-} \rightarrow \nu_{\mathrm{L}} \mathrm{K}^{*^{-}}$ & 1.22 \\
\hline $\mathrm{L}^{-} \rightarrow \nu_{\mathrm{L}} \mathrm{a}_{1}^{-}$ & 0.99 \\
\hline $\mathrm{L}^{-} \rightarrow \nu_{\mathrm{L}} \overline{\mathrm{u} d}$ & 0.74 \\
\hline $\mathrm{L}^{-} \rightarrow \nu_{\mathrm{L}} \overline{\mathrm{cs}}$ & 0.96 \\
\hline $\mathrm{L}^{-} \rightarrow \nu_{\mathrm{L}} \tau^{-} \bar{\nu}_{\tau}$ & 0.96 \\
\hline
\end{tabular}

pion mass. Figure A.6 shows a typical $\left(\mathrm{M}_{\mathrm{L}}=10 \mathrm{GeV}\right)$ magnified view for the range $0<\delta \mathrm{M}<4.0 \mathrm{GeV}$ in which individual decay modes are labelled clearly. The rapid drop of $\mathrm{B}\left(\mathrm{L}^{-} \rightarrow \nu_{\mathrm{L}} \mathrm{e}^{-} \bar{\nu}_{\mathrm{e}}\right)$ and rise from zero of $\mathrm{B}\left(\mathrm{L}^{-} \rightarrow \nu_{\mathrm{L}} \pi^{-}\right)$at the charged pion mass is magnified further in fig. A.7. 


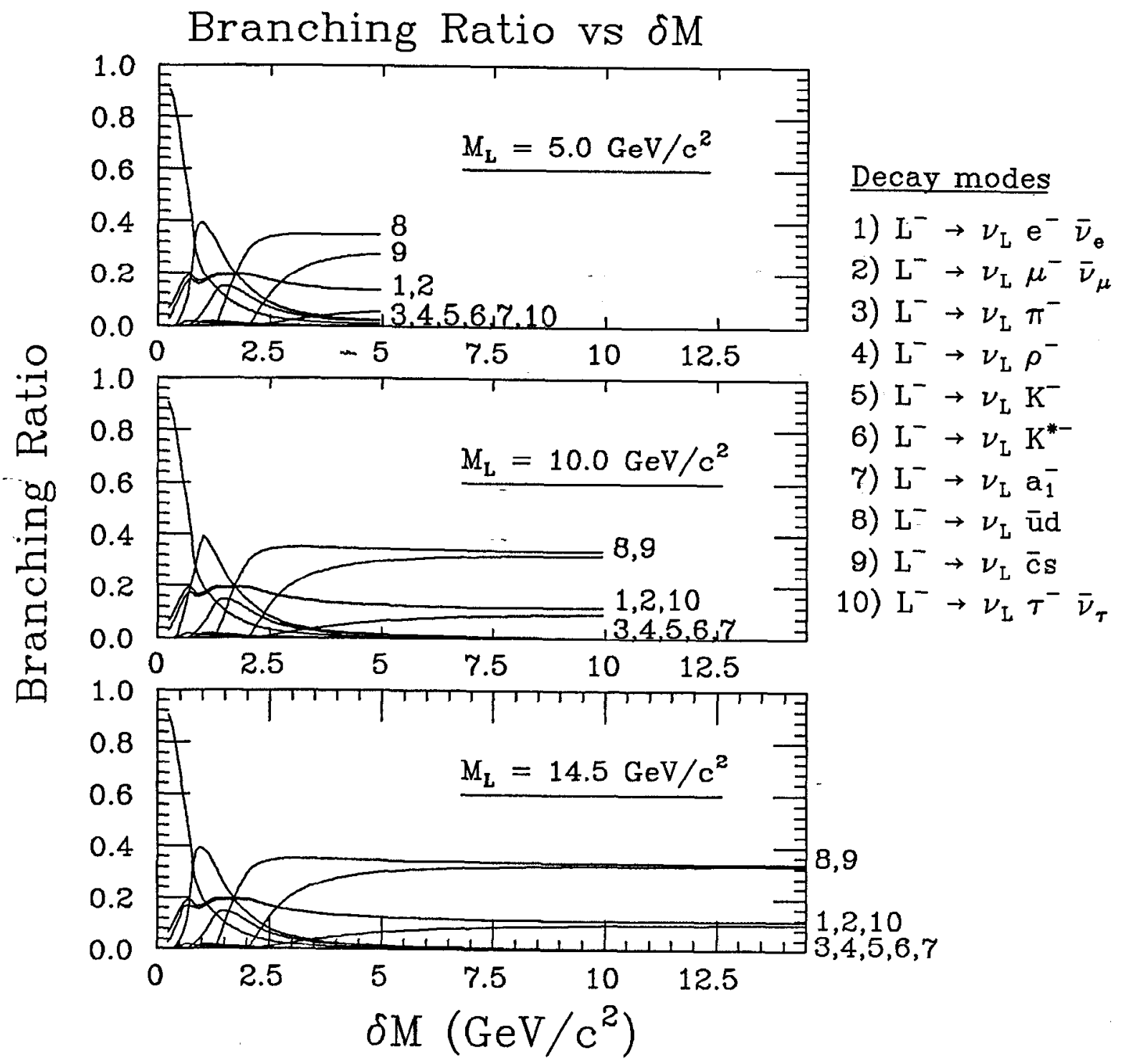

Figure A.5. Calculated branching ratios vs $M_{L}-M_{\nu_{L}}$ for three representative values of $M_{L}$. The branching ratios are quite sensitive to the mass difference when the difference is small and relatively insensitive to $\mathrm{M}_{\mathrm{L}}$ for fixed $\delta \mathrm{M}$. For large $\delta \mathrm{M}$, branching ratios are stable as the three-body leptonic and hadronic continuum widths approach their asymptotic forms. 


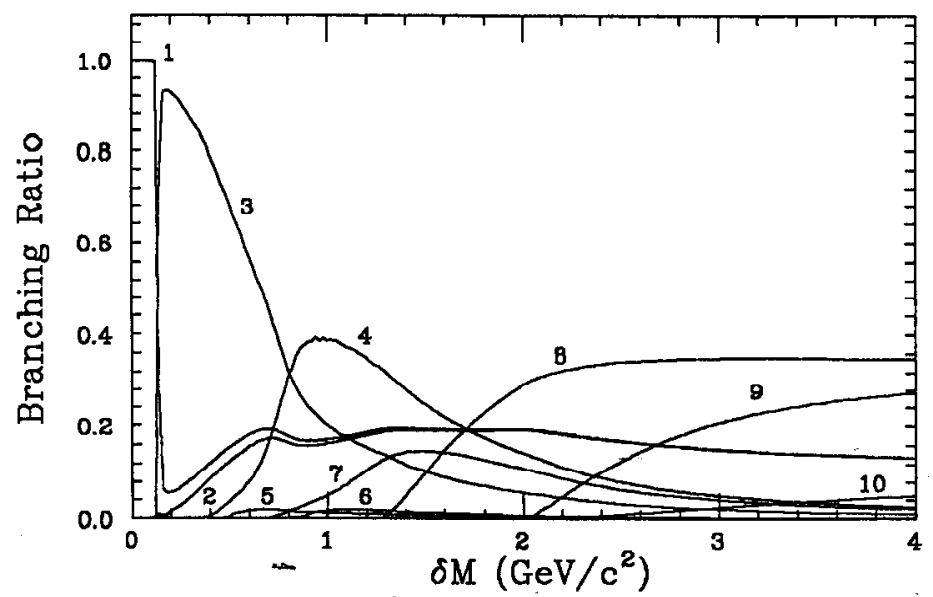
1) $\mathrm{L}^{-} \rightarrow \nu_{L} \mathrm{e}^{-} \bar{\nu}_{\mathrm{g}}$
2) $L^{-} \rightarrow \nu_{L} \mu^{-} \bar{\nu}_{\mu}$
3) $\mathrm{L}^{-} \rightarrow \nu_{\mathrm{L}} \pi^{-}$
4) $\mathrm{L}^{-} \rightarrow \nu_{\mathrm{L}} \rho^{-}$
5) $\mathrm{L}^{-} \rightarrow \nu_{\mathrm{L}} \mathrm{K}^{-}$
6) $\mathrm{L}^{-} \rightarrow \nu_{\mathrm{L}} \mathrm{K}^{*-}$
7) $\mathrm{L}^{-} \rightarrow \nu_{\mathrm{L}} \mathrm{a}_{1}^{-}$
9) $\mathrm{L}^{-} \rightarrow \nu_{\mathrm{L}} \overline{\mathrm{cs}}$
8) $\mathrm{L}^{-} \rightarrow \nu_{\mathrm{L}} \overline{\mathrm{u}} \mathrm{d}$
10) $\mathrm{L}^{-} \rightarrow \nu_{\mathrm{L}} \tau^{-}$

Figure A.6. Calculated branching ratios vs mass difference for $\mathrm{M}_{\mathrm{L}}=10.0 \mathrm{GeV}$.

B.R. vs $\delta \mathrm{M}\left(\mathrm{M}_{\mathrm{L}}=10.0 \mathrm{GeV} / \mathrm{c}^{2}\right)$
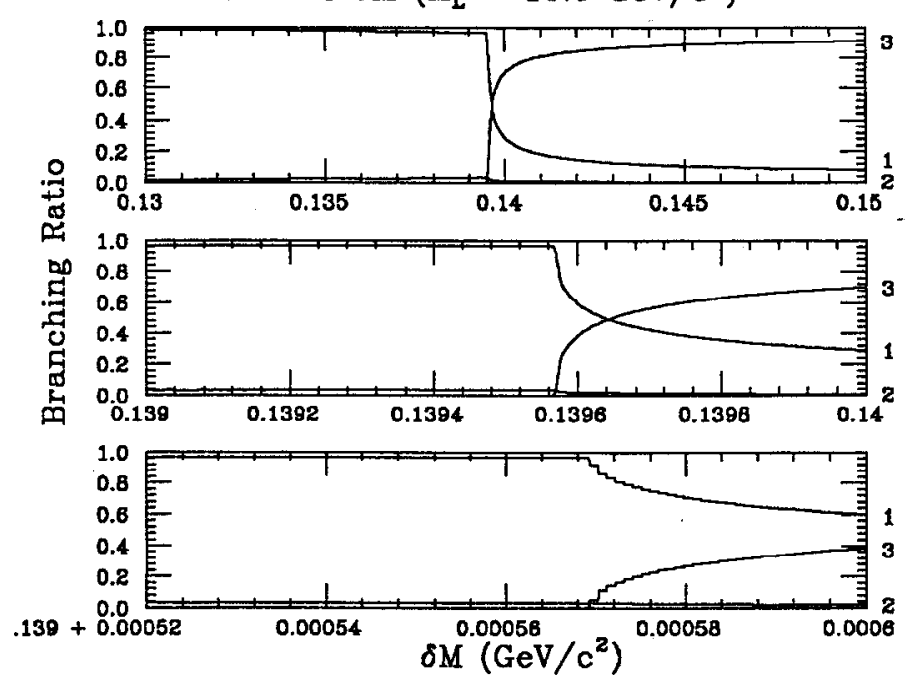
1) $\mathrm{L}^{-} \rightarrow \nu_{\mathrm{L}} \mathrm{e}^{-} \bar{\nu}_{\mathrm{e}}$
2) $\mathrm{L}^{-} \rightarrow \nu_{1} \mu^{-} \bar{\nu}_{\mu}$
3) $\mathrm{L}^{-} \rightarrow \nu_{\mathrm{L}} \pi^{-}$

Figure A.7. Calculated branching ratios vs mass difference for $\mathrm{M}_{\mathrm{L}}=10.0 \mathrm{GeV}$ at three magnifications of the mass scale. Once the mass difference exceeds the charged pion mass threshold, the pion decay width rapidly surpasses the electron width and remains dominant until mass differences comparable to the charged rho mass are reached. 


\section{References}

1. G. Altarelli, lecture given at Cargèse Summer Institute, 1985, CERN-TH-433385 .

2. M. Gell-Mann, P. Ramond, and R. Slansky, in Supergravity, ed. P. Van Nieuwenhuizen and D.Z. Freedman, North-Holland, Amsterdam, 1979; R.N. Mohapatra and G. Senjanovic, Phys. Rev. Lett. 44, 912 (1980).

3. M.L. Perl, in Proceedings of the XXIII International Conference on High Energy Physics, p. 596, Berkeley,..California, 1986.

4. C. Albajar et al., Phys. Lett. 185B, 241 (1987).

5. R.M. Barnett and H.E. Haber, Phys. Rev. D36, 2042 (1987).

6. G.N. Kim et al., to appear in Proceedings of the XXIV International Conference on High Energy Physics, Munich, 1988, KEK-PREPRINT-88-42.

7. I. Adachi et al., Phys. Rev. D37, 1339 (1988).

8. K. Abe et al., Phys. Rev. Lett. 61, 915 (1988).

9. D. Stoker et al.; to appear in Physical Review D, JHU-HEP-88-0301, ST,ACPUB-4590.

10. L. Mathis, Ph.D. thesis, University of California, Berkeley, LBL-25261 (1988).

11. J.B. Kogut, Rev. Mod. Phys. 55, 775 (1983).

12. M. Kobayashi and T. Maskawa, Prog. Theor. Phys.(Kyoto) 49, 652 (1973).

13. D.L. Burke, talk given at Theoretical Advanced Study Institute in Particle Physics, Santa Cruz, Calif., 1986.

14. C. Hearty et al., SLAC-PUB-4684 (1988).

15. W.T. Ford et al., Phys. Rev. D33, 3472 (1986). 
16. H.J. Behrend et al., DESY-88-052 (1988).

17. G. Steigman et al., Phys. Lett. 176B, 33 (1986).

18. J. Ellis, K. Enqvist, D.V. Nanopoulos, and S. Sarkar, Phys. Lett. 167B, 457 (1986).

19. R. Cowsik and J. McClelland, Phys. Rev. Lett. 29, 669 (1972).

20. B.W. Lee and S. Weinberg, Phys. Rev. Lett. 39, 165 (1977).

21. J.E. Gunn et al., Astrophys. J. 2231015 (1978).

22. H. Harari, in Proceeding of Neutrino Conference, Boston, Massachusetts, June, 1988.

23. S. Raby and G.B. West, Nuc. Phys. B292, 793 (1987).

24. R. Davis, B.T. Cleveland, and J.K. Rowley, in Proceedings, Intersections Between Particle and Nuclear Physics, Steamboat Springs, 1984.

25. Y. Totsuka, to appear in Proceedings of the XXIV International Conference on High Energy Physics, Munich, 1988, UT-ICR-181-88-27. .

26. J.R. Primack, D. Seckel, and B. Sadoulet, Ann. Rev. Nuc. Part. Sci. 38, (1988).

27. M.L. Perl et al., Phys. Rev. Lett. 35, 1489 (1975).

28. M. Nelson, Ph.D. thesis, University of California, Berkeley, LBL-16724 (1983).

29. W. Bartel et al., Z. Phys. 29C, 505 (1985).

30. A more detailed description of both the history of weak interaction theory and of our current understanding of it can be found in E.D. Commins and P.H. Bucksbaum, Weak Interaction of Leptons and Quarks, Cambridge, 1983, Cambridge University Press.

31. W. Pauli, Proc. VII Solvay Congress, Brussels, p. 324, Paris, 1933, GauthierVillars.

32. E. Fermi, Z. Phys. 88, 161 (1934). 
33. T.D. Lee and C.N. Yang, Phys. Rev. 104, 254 (1956).

34. C.S. Wu et al., Phys. Rev. 105, 1413 (1957).

35. R.P. Feynman and M. Gell-Mann, Phys. Rev. 109, 193 (1958).

36. J.D. Bjorken and S.D. Drell, it Relativistic Quantum Mechanics, New York, 1964, McGraw-Hill.

37. P.W. Higgs, Phys. Lett. 12, 132 (1964). P.W. Higgs, Phys. Lett. 13, 508 (1964). P.W. Higgs, Phys. Rev. 145, 1156 (1966).

38. S.L. Glashow, Nuc. Phys. B22, 579 (1961). S. Weinberg, Phys. Rev. Lett. 19, 1264 (1967). A. Salam, Nobel Symposium, No. 8, p. 367, ed. N. Svartholm, Stockholm, 1968 Almquist and-Wiksell.

39. M. Banner et al., Phys. Lett. 122B, 476 (1983).

40. P. Bagnaia et al., Phys. Lett. 129B, 130 (1983).

41. B. Barish, R. Stroynowski, Phys. Reports 157, 1 (1988).

42. T. Sjöstrand, Comp. Phys. Comm. 39, 347 (1986). A. Bäcker, Z. Phys. C12, 161 (1982).

43. F.A. Berends et al.., Acta Phys. Pol. B14 413 (1983).

44. G. Bonneau and F. Martin, Nuc. Phys. B27, 381 (1971).

45. F.A. Berends and R. Kleiss, Nuc. Phys. B177, 237 (1981).

46. Y.S. Tsai, presented at The Asia Pacific Physics Conference, June 12-18, 1983, SI.AC-PUB-3129.

47. F.A. Berends and R. Gastmans, in Electromagnetic Interactions of Hadrons, vol. 2, p. 471, ed. A. Donnachie and G. Shaw (Plenum, 1978).

48. R. H. Schindler et al, Phys. Rev. D24, 78 (1981).

49. B. LeClaire, Ph.D. thesis, Stanford University, SL $\Lambda$ C-321 (1987).

50. P. Rowson, Ph.D. thesis, University of California, Berkeley, LBL-20463 (1985). 
51. C. Blocker, Ph.D. thesis, University of California, Berkeley, LBL-10801 (1980).

52. R.L. Ford and W.R. Nelson, computer code EGS Version 3, Stanford Linear Accelerator Center, SLAC-PUB-0210 (1978).

53. D. Karlen, Ph.D. thesis, Stanford University, SLAC-325 (1988).

54. F. James and M. Roos, Comp. Phys. Comm. 10343 (1985).

55. W.B. Atwood et al., SLAC-TN-76-7 (1976).

56. H. Brafman et al., SLAC-PUB-2033 (1977).

57. J. Dillon, VFINDP Vee finder, Trilling-Goldhaber Group physics note \#232, ... March 1982.

58. G. Molière, in Cosmic Radiation, ed. W. Heisenberg, Dover, New York, 1946. J. Nishimura and K. Kamata, Prog. Theor. Phys.(Kyoto) 7, 1952.

59. F. Gilman, S. Rhie, Phys. Rev. D31, 1066 (1985).

60. J.R. Smith, Ph.D. thesis, University of California, Davis, RX-1171 (1982).

61. R.H. Dalitz and D.R. Yennie, Phys. Rev. 105, 1598 (1957)

62. S.J. Brodsky, T. Kinoshita, and H. Terazawa, Phys. Rev. D4, 1532 (1971).

63. C. Berger and W. Wagner, Phys. Rep. 146, 1 (1987).

64. H. Kolanoski, in Proceedings of the $V$ International Colloquium on PhotonPhoton Interactions, Aachen, 1983, Springer Lecture Notes in Physics, v. 191.

65. H. Albrecht et al., DESY 88-150.

66. F.E. Low, Phys. Rev. 120, 582 (1960).

67. J. Dorfan, internal Mark II memo, April 4, 1979.

68. Particle Data Group, Phys. Lett. 204B, 1 (1988).

69. G. Bonvicini et al., in Proceedings of the Third Mark II Workshop on SLC Physics, p. 546 (1987). 
70. G. Bonvicini and L. Trentadue, submitted to Nuc. Phys. B, UM-HE-88-36. (1988).

71. D. Stoker, Mark II /SLC Note \# 161, Nov. 25, 1986.

72. B.F.L. Ward, Phys. Rev. D35, 2092 (1987). See also AIP document PAPS PRVDA-35-2092-80 which contains detailed appendices. Ward gives general formulas for the production and decay of gauge-Higgs fermions associated with a minimal supersymmetric extension of the standard model. It is straightforward to derive from these formulas the expressions presented here.

73. R. Shrock, Phys. Rev. D24, 1275 (1981).

74. L. Okun, Leptons and Quarks (North-Holland, Amsterdam, The Netherlands, 1982) pp. 107-109.

75. S. Jadach, "TAUOLA-The library of the Monte Carlo programs simulating decays of polarized $\tau$ leptons," in preparation.

76. M. Perl, Nuc. Phys. B, Proceedings Supplements: Second Topical Seminar on Heavy Flavors, San Miniato, Italy, May 25-29, 1987.

77. P. Burchat et al., Phys. Rev. D35, 27 (1987).

78. D. Hitlin, CALT-68-1472, in Proceedings of the 1987 International Symposium on Lepton and Photon Interactions at High Energies, Hamburg, Germany. 